\title{
Emulsion Templating: Unexpected Morphology of Monodisperse Macroporous Polymers
}

\author{
Von der Fakultät Chemie der Universität Stuttgart \\ zur Erlangung der Würde eines Doktors der \\ Naturwissenschaften (Dr. rer. nat.) genehmigte Abhandlung \\ Vorgelegt von \\ Lukas Koch \\ aus Anger \\ Hauptberichterin: Prof. Dr. Cosima Stubenrauch \\ Mitberichter: $\quad$ Prof. Dr. Michael D. Gilchrist \\ Prüfungsvorsitzender: Prof. Dr. Michael R. Buchmeiser
}

Tag der mündlichen Prüfung: 31.03.2021

Institut für Physikalische Chemie

der Universität Stuttgart

2021 

für Conny und Franz Koch 



\section{Acknowledgements}

First and foremost, I would like to express my deep gratitude to Prof. Dr. Cosima Stubenrauch for giving me the opportunity to do my $\mathrm{PhD}$ thesis under her outstanding supervision. She guided me in the beginning of my research when experiments had to be planned and later played a vital role in the discussion of the results. Because of her advice, I was able to improve my writing skills and became a better scientist in general. Moreover, I am thankful for all the national and international conferences I was able to attend because of her support.

Furthermore, I would like to thank Prof. Dr. Michael D. Gilchrist for taking on the role as the second examiner of my $\mathrm{PhD}$ thesis and Prof. Dr. Michael R. Buchmeiser for being my GRADUS mentor as well as accepting the chairmanship of my $\mathrm{PhD}$ defense.

I would like to further express my gratitude to Dr. Wiebke Drenckhan who greatly assisting my research with uncountable fruitful discussions. Moreover, I would like to thank her and her whole research group for welcoming me in Strasbourg when I was residing with them. Special thanks to Dr. Sebastian Andrieux for supporting me as my "local contact" in Strasbourg.

I am also very grateful to all the people who supported my work over these recent years. I am thankful to Prof. Dr. Alexander Fels and Dr. Yaseen Qawasmi for their support during the SEM measurements, to Apl. Prof. Dr. Thomas Sottmann, Diana Zauser, Shih-Yu Tseng, Karina Abitaev, and Julian Fischer for their roles in the SANS measurements, to Maximilian Krappel for his help with the DLS measurements, and to Dr. Natalie Preisig for recording the FFEM pictures. I also want to thank the CTAs Birgit Feucht and Diana Zauser as well as the staff of the electrical, mechanical, and glass workshops for their support in the lab. I would like to further express my gratitude to my students Sabrina Beyer, Benjamin Kordes, Sophia Botsch, and Manfred Stenzel who greatly assisted my $\mathrm{PhD}$ thesis with their work. Moreover, I want to give a big "thank you" to Miriam Dabrowski for supporting my work by her contributions in the lab and office. In general, I also want thank all former and current members of the Stubenrauch and Sottmann groups for the pleasant atmosphere during my time in Stuttgart.

To Clarissa Dietrich, Julian Fischer, and all the other people who practiced sports with me, I want to express my gratitude for helping me achieve a work-life-balance during my $\mathrm{PhD}$ thesis.

Finally, I want to thank my friends and family at home who supported me during both my studies and my PhD thesis. My parents Cornelia and Franz Koch deserve the greatest gratitude, because without their never-ending support, neither my studies nor my $\mathrm{PhD}$ thesis would have been possible. 


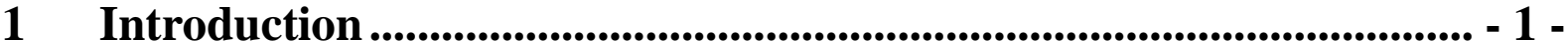

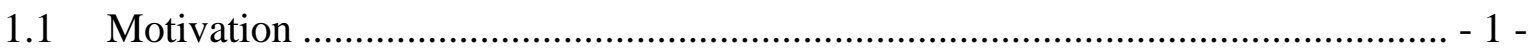

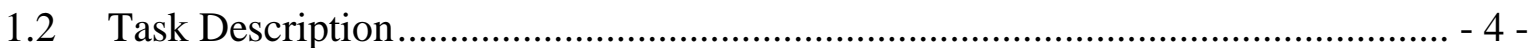

2 Theoretical Background ................................................................................... - 7 -

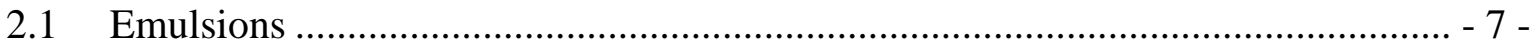

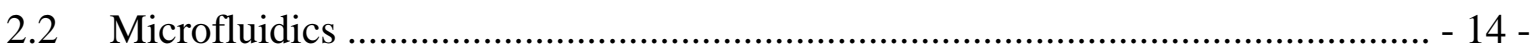

2.3 Polymers and Porous Polymers ….............................................................. - 23 -

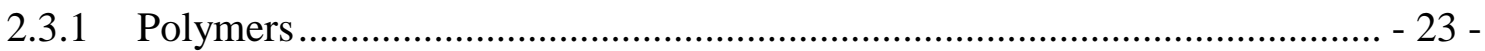

2.3.2 Porous Polymers......................................................................................... 30 -

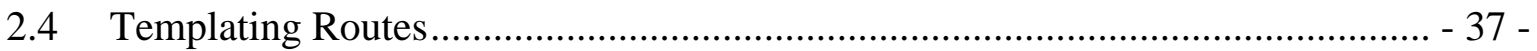

3 Results and Discussion ..................................................................... 43 -

3.1 Disproof of Osmotic Transport...................................................................... - 43 -

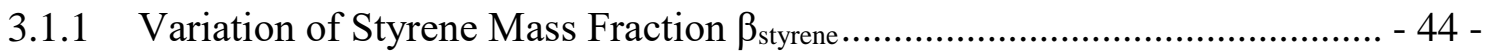

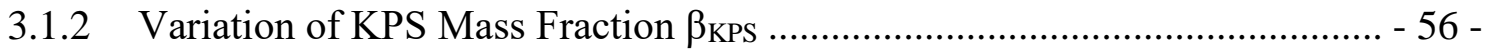

3.2 New Mechanism: Surfactant Diffusion ............................................................... - 65 -

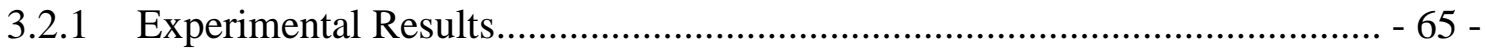

3.2.2 Discussion of Experimental Results ........................................................ - 81 -

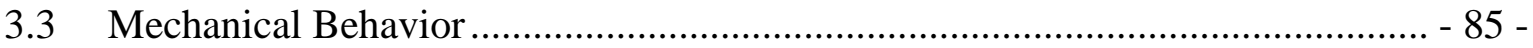

4 Conclusions and Outlook............................................................................... - 95 -

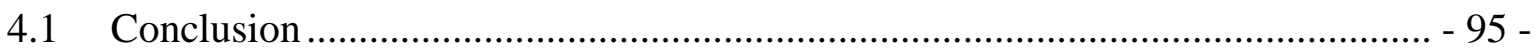

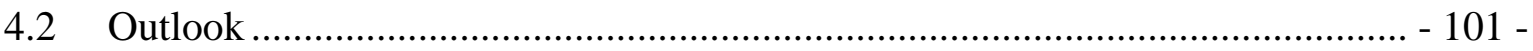

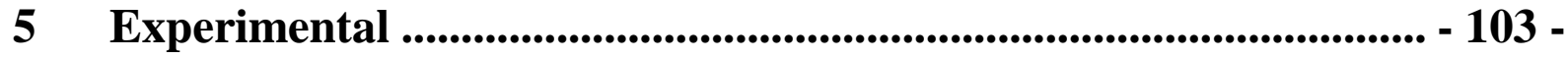

5.1 Materials .......................................................................................... - 103 -

5.2 Synthesis of Macroporous PS/polyDVB …................................................... 103 -

5.3 Analysis of Macroporous PS/polyDVB …................................................... - 105 -

5.3.1 Analysis of Morphology....................................................................... - 105 -

5.3.2 Analysis of Mechanical Behavior …....................................................... - 106 - 


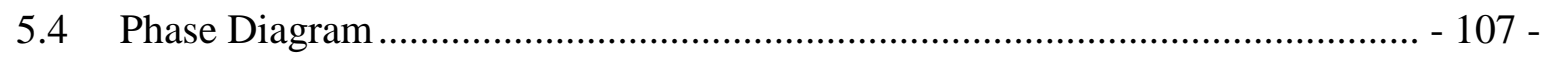

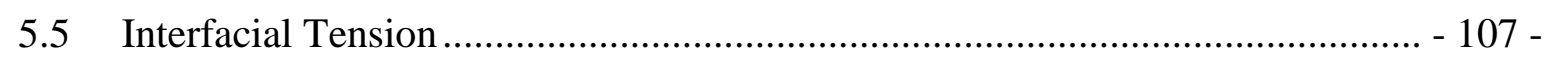

5.6 Small angle neutron scattering ............................................................. - 107 -

5.7 Freeze Fracture Electron Microscopy ...................................................... - 108 -

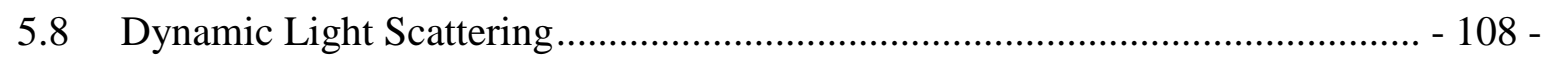

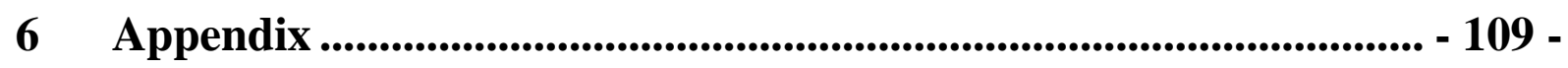

6.1 Supplementary Calculations ................................................................... 109 -

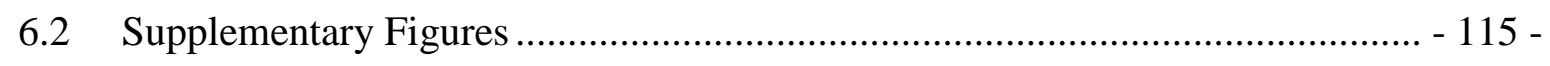

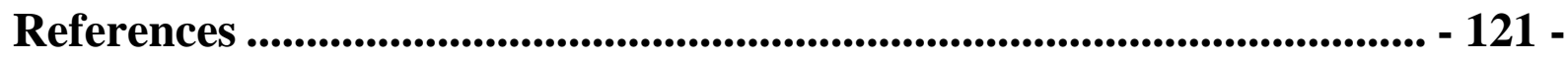




\section{Abbreviations and Symbols}

\section{Abbreviations}

$2 \mathrm{D}$

two dimensional

$3 \mathrm{D}$

three dimensional

AIBN azobisisobutyronitrile

APD avalanche photodiode

CFCs chlorofluorocarbons

cont continuous (phase)

$\mathrm{D}_{2} \mathrm{O} \quad$ deuterated water

disp dispersed (phase)

DLS dynamic light scattering

DNA deoxyribonucleic acid

DSC differential scanning calorimetry

DTA differential thermal analysis

DVB divinylbenzene

EPS expanded polystyrene

ETFE ethylene tetrafluoroethylene

FEP fluorinated ethylene propylene

FFEM freeze fracture electron microscopy/microscope

g/o gas-in-oil

g/w gas-in-water

HIPE high internal phase emulsion

IUPAC International Union of Pure and Applied Chemistry

KPS potassium peroxodisulfate

LAS linear alkylbenzene sulphonate 


\begin{tabular}{|c|c|}
\hline LIPE & low internal phase emulsion \\
\hline MIPE & medium internal phase emulsion \\
\hline $\mathrm{mol} \%$ & mol percent \\
\hline $\mathrm{n}$ & unspecified, large number \\
\hline $\mathrm{o} / \mathrm{w}$ & oil-in-water (emulsion) \\
\hline $\mathrm{o} / \mathrm{w} / \mathrm{o}$ & oil-in-water-in-oil (emulsion) \\
\hline PEG & polyethylene glycol \\
\hline$P D I$ & polydispersity index \\
\hline PDMS & polydimethylsiloxane \\
\hline $\mathrm{PE}$ & polyethylene \\
\hline PET & polyethylene terephthalate \\
\hline $\mathrm{Ph}$ & Phenyl rest \\
\hline $\mathrm{PhD}$ & philosophiae doctor (Doctor of Philosophy) \\
\hline PMMA & poly(methyl methacrylate) \\
\hline polyDVB & polydivinylbenzene \\
\hline polyHIPE & polymerized high internal phase emulsion \\
\hline PS & polystyrene \\
\hline PVC & polyvinyl chloride \\
\hline $\mathrm{R}$ & aliphatic rest \\
\hline SANS & small angle neutron scattering \\
\hline SEI & secondary electron imaging \\
\hline SEM & scanning electron microscopy/microscope \\
\hline TEM & transmission electron microscopy/microscope \\
\hline UV & ultraviolet \\
\hline vol\% & volume percent \\
\hline
\end{tabular}




$\begin{array}{ll}\text { wt.\% } & \text { weight percent } \\ \text { w/o } & \text { water-in-oil } \\ \text { w/o/w } & \text { water-in-oil-in-water } \\ \text { XPS } & \text { extruded polystyrene }\end{array}$

\section{Constants, Numbers and Units}

$\begin{array}{ll}\AA & \text { ångström }\left(10^{-10} \mathrm{~m}\right) \\ g & \text { gravitational constant of earth }\left(9.81 \mathrm{~m} \mathrm{~s}^{-2}\right) \\ \mathrm{N} & \text { Newton }\left(\mathrm{kg} \mathrm{m} \mathrm{s}^{-2}\right) \\ N_{\mathrm{A}} & \text { Avogadro number }\left(6.022 \cdot 10^{23} \mathrm{~mol}^{-1}\right) \\ \mathrm{Pa} & \text { Pascal }\left(\mathrm{N} \mathrm{m}^{-2}\right)\end{array}$

\section{Greek Symbols}

$\beta_{\mathrm{i}} \quad$ mass fraction of component $\mathrm{i} / \mathrm{wt} \%$

$\gamma \quad$ interfacial tension $/ \mathrm{mN} \mathrm{m}^{-1}$

$\Delta h \quad$ height difference / $\mathrm{m}$

$\Delta p \quad$ pressure difference / $\mathrm{N} \mathrm{m}^{-2}$

$\varepsilon \quad$ strain / \%

$\theta \quad$ angle at which two channels meet in microfluidic cross-flow geometry $/^{\circ}$

$\eta_{\mathrm{i}} \quad$ dynamic viscosity of component $\mathrm{i} / \mathrm{kg} \mathrm{m}^{-1} \mathrm{~s}^{-1}$

$\kappa \quad$ curvature $/ \mathrm{m}^{-1}$

$\lambda \quad$ viscosity ratio

$v^{*} \quad$ Poisson's ratio

$\rho_{\mathrm{i}} \quad$ density of component $\mathrm{i} / \mathrm{kg} \mathrm{m}^{-3}$ or $\mathrm{g} \mathrm{cm}^{-3}$

$\rho_{\text {rel }} \quad$ relative density 

stress / N
$\sigma_{\mathrm{ys}} \quad$ yield strength / $\mathrm{MPa}$
$\varphi \quad$ flow ratio
$\Phi_{\mathrm{i}} \quad$ volume fraction of component i / vol\%
$\chi_{\mathrm{i}} \quad$ molar fraction of component i / mol\%

\section{Roman Symbols}

\begin{tabular}{|c|c|}
\hline$A_{\mathrm{i}}$ & area of object i / $\mathrm{m}^{2}$ \\
\hline$A_{\text {interface }}$ & interfacial area $/ \mathrm{m}^{2}$ \\
\hline Bo & Bond number \\
\hline $\mathrm{Ca}$ & Capillary number \\
\hline$C_{\mathrm{C}} / C_{1} / C_{1}^{\prime}$ & proportionality constants \\
\hline$C_{\mathrm{i}}$ & circumference of object i / m \\
\hline$d_{\text {average }}$ & average diameter / $\mathrm{m}$ \\
\hline$d_{\mathrm{i}}$ & diameter of object i / m \\
\hline$d_{\mathrm{sd}}$ & standard deviation of diameter / $\mathrm{m}$ \\
\hline$E_{\mathrm{i}}$ & Young's Modulus of component i / MPa \\
\hline$F$ & force / $\mathrm{N}$ \\
\hline$F_{\max }$ & $\begin{array}{l}\text { point at which the linear elastic region goes over into the plateau region in stress- } \\
\text { strain-curves / N }\end{array}$ \\
\hline$f_{\mathrm{c}}$ & force of capillary pressure $/ \mathrm{kg} \mathrm{m}^{-1} \mathrm{~s}^{-2}$ \\
\hline$f_{\mathrm{g}}$ & gravitational force $/ \mathrm{kg} \mathrm{m}^{-1} \mathrm{~s}^{-2}$ \\
\hline$f_{\mathrm{i}}$ & inertial force $/ \mathrm{kg} \mathrm{m}^{-1} \mathrm{~s}^{-2}$ \\
\hline$f_{\mathrm{v}}$ & viscous force $/ \mathrm{kg} \mathrm{m}^{-1} \mathrm{~s}^{-2}$ \\
\hline$G$ & Gibbs free energy / J \\
\hline
\end{tabular}




\begin{tabular}{|c|c|}
\hline$h$ & height / $\mathrm{m}$ \\
\hline$h_{0}$ & initial height / $\mathrm{m}$ \\
\hline$H$ & enthalpy / J \\
\hline$I$ & measured intensity in SANS \\
\hline$I(q)$ & calculated intensity in SANS \\
\hline$L$ & characteristic length scale of microfluidic chip / $\mathrm{m}$ \\
\hline$p$ & pressure / $\mathrm{N} \mathrm{m}^{-2}$ \\
\hline$p_{0}$ & initial (gas) pressure / $\mathrm{N} \mathrm{m}^{-2}$ \\
\hline$P$ & porosity / \% \\
\hline$P(q)$ & form factor in SANS \\
\hline$q$ & scattering vector / $\AA^{-1}$ \\
\hline$Q_{\mathrm{i}}$ & flow rate of component $\mathrm{i} / \mathrm{m}^{3} \mathrm{~s}^{-1}$ \\
\hline$r_{\mathrm{i}}$ & radius of object $\mathrm{i} / \mathrm{m}$ \\
\hline$r_{\text {Hyd }}$ & hydrodynamic radius / $\mathrm{m}$ \\
\hline$R e$ & Reynolds number \\
\hline$S$ & shape factor \\
\hline$S(q)$ & structure factor in SANS \\
\hline$S_{\mathrm{td}}$ & thermodynamic entropy / J K $\mathrm{K}^{-1}$ \\
\hline$t_{\mathrm{i}}$ & thickness of object i / m \\
\hline$T$ & temperature / $\mathrm{K}$ or ${ }^{\circ} \mathrm{C}$ \\
\hline$T_{\mathrm{g}}$ & glass transition temperature / $\mathrm{K}$ or ${ }^{\circ} \mathrm{C}$ \\
\hline$T_{\mathrm{m}}$ & melting temperature / $\mathrm{K}$ or ${ }^{\circ} \mathrm{C}$ \\
\hline$v_{\mathrm{i}}$ & velocity of component $\mathrm{i} / \mathrm{m} \mathrm{s}^{-1}$ \\
\hline$V_{\mathrm{i}}$ & volume of component $\mathrm{i} / \mathrm{m}^{3}$ \\
\hline$W e$ & Weber number \\
\hline
\end{tabular}




\begin{abstract}
The polymerization and drying of monodisperse water-in-styrene/divinylbenzene (DVB) high internal phase emulsions (HIPEs) leads to monodisperse macroporous polystyrene (PS)/polydivinylbenzene (polyDVB). When the monomer-soluble azobisisobutyronitrile (AIBN) is used as initiator, spherical and interconnected pores and porous pore walls are obtained. In contrast, when the water-soluble potassium peroxodisulfate (KPS) is used, polyhedral and closed pores are obtained and the pore walls are comprised of two similar looking outer layers and one different inner layer. The aim of this work was to identify the mechanism (1) that transforms spherical droplets into polyhedral pores and (2) that creates a three-layered pore wall when the polymerization is initiated from the water/monomer interface with KPS.
\end{abstract}

The styrene/DVB mass ratio and the KPS mass fraction were varied to test the existing hypothesis, i.e. an osmotic transport of DVB. Scanning electron microscopy (SEM) pictures revealed that the morphology of the samples does not change in the way it is expected if osmotic transport of DVB was the acting mechanism. Therefore, the existing hypothesis was rejected and a new explanation had to be found. Experiments in which the surfactant mass fraction $\beta_{\text {surfactant }}$ was varied revealed that the relative size of the inner layer increases and the relative size of the outer layers decreases when $\beta_{\text {surfactant }}$ is increased. Moreover, it was found that the outer layers are non-porous and that the inner layer is porous. With the help of a model ternary phase diagram consisting of styrene, surfactant, and PS, it was shown that the surfactant is not soluble in partially polymerized styrene/PS mixtures. The experimental results allow suggesting a mechanism that is based on surfactant diffusion. Since the polymerization starts at the water/monomer interface with KPS, a partially polymerized layer forms close to the interface. From this layer, surfactant molecules that are dissolved in the continuous phase diffuse either (1) to the water/monomer interface or (2) to the interior of the continuous phase. (1) Surfactant diffusion to the interface induces an overpopulation of surfactant. This enables the interface to increase its area, which, in turn, transforms the spherical droplets to polyhedral pores. (2) Surfactant diffusion to the interior of the continuous phase leads to an accumulation of surfactant, while the regions close to the interface become surfactant-free. When the surfactant is washed out during purification, a porous inner and two non-porous outer layers are obtained.

Additionally, the mechanical properties of monodisperse macroporous PS/polyDVB were investigated. It was found that the samples are only elastomeric when the amount of DVB is low, while they are elastic-brittle for all other monomer compositions. 


\section{Kurzzusammenfassung}

Durch Polymerisation und Trocknen wird aus monodispersen Wasser-in-Styrol/Divinylbenzol (DVB) Emulsionen monodisperses, makroporöses Polystyrol (PS)/Polydivinylbenzol. Mit monomerlöslichem Azobis(isobutyronitril) (AIBN) als Initiator werden sphärische und offene Poren sowie poröse Porenwände erhalten. Dagegen entstehen mit wasserlöslichem Kaliumperoxodisulfat (KPS) polyedrische und geschlossene Poren und die Porenwände bestehen aus zwei ähnlich aussehenden äußeren Schichten und einer inneren Schicht. Das Ziel dieser Arbeit war es, den Mechanismus zu identifizieren, (1) der sphärische Tropfen in polyedrische Poren verwandelt und (2) der eine dreilagige Porenwand erzeugt, wenn die Polymerisation durch KPS an der Wasser/Monomer Grenzfläche beginnt.

Das Styrol/DVB Massenverhältnis und der KPS Massenbruch wurden variiert, um die bestehende Hypothese zu überprüfen, die auf osmotischen Transport von DVB basiert. Rasterelektronenmikroskopie (REM) Aufnahmen zeigten, dass sich die Morphologie der Proben nicht in der Art verändert, wie sie es tun sollte, wenn osmotischer Transport von DVB der wirkende Mechanismus ist. Somit wurde die bestehende Hypothese verworfen und eine neue Erklärung musste gefunden werden. In Experimenten, in denen der Tensidmassenbruch $\beta_{\text {Tensid }}$ erhöht wurde, nahm die relative Größe der inneren Schicht zu und die relative Größe der äußeren Schichten ab. Zudem wurde herausgefunden, dass die äußeren Schichten nicht porös sind, sehr wohl aber die innere Schicht. Mithilfe eines ternären Phasendiagrams bestehend aus Styrol, Tensid und PS wurde gezeigt, dass das Tensid nicht in partiell polymerisiertem Styrol/PS löslich ist. Der anhand der experimentellen Ergebnisse vorgeschlagene Mechanismus basiert auf Tensiddiffusion. Da die Polymerisation durch KPS an der Wasser/Monomer Grenzfläche beginnt, entsteht in deren Nähe eine partiell polymerisierte Schicht. In der kontinuierlichen Phase gelöste Tensidmoleküle diffundieren aus dieser Schicht entweder (1) zur Grenzfläche oder (2) ins Innere der kontinuierlichen Phase. (1) Tensiddiffusion zur Grenzfläche verursacht dort eine Übersättigung, wodurch sich die Grenzfläche vergrößern kann und aus sphärischen Tropfen polyedrische Poren werden. (2) Tensid Diffusion ins Innere der kontinuierlichen Phase verursacht dort eine Anreicherung mit Tensid, während nahe der Grenzfläche kein Tensid mehr vorhanden ist. Wenn das Tensid während der Reinigung ausgewaschen wird, entstehen eine poröse innere und zwei nicht-poröse äußere Schichten.

Zusätzlich wurden die mechanischen Eigenschaften von monodispersen, makroporösem PS/polyDVB untersucht. Dieses ist nur dann elastomerisch, wenn der DVB Anteil niedrig ist, während es bei allen anderen Monomerzusammensetzungen elastisch-brüchig ist. 


\section{Introduction}

\subsection{Motivation}

Polymers have been used by humans indirectly or directly for a long time in the form of biopolymers like proteins, polysaccharides, and deoxyribonucleic acid (DNA). The discovery of synthetic polymers in the $19^{\text {th }}$ century has fundamentally changed modern society. Since 1909 , when the first industrially produced plastic, Bakelite [Bae09], was introduced, a wide range of products has been made from synthetic polymers. Among these products are, for example, porous polymers where the dispersed phase is a gas and the continuous phase is a polymer. They are employed in a broad variety of areas ranging from construction (insulation material) and vehicles (dashboard) to sports (protective gear) and hygiene (polyurethane sponges), to mention just a few. Compared with their bulk counterparts, one big advantage of porous polymers is the fact that the density $\rho$ can be adjusted by changing the amount of the dispersed gas. The gamut of densities includes not only the dense bulk polymer itself where the volume percentage of gas is very small ( $\rho_{\text {porous polymer }} \sim 10^{3} \mathrm{~kg} \mathrm{~m}^{-3}$ ), but also materials that consist almost entirely of gas ( $\rho_{\text {porous polymer }} \sim 10^{0} \mathrm{~kg} \mathrm{~m}^{-3}$ ) [Lan95]. As the mechanical properties of porous polymers strongly depend on their density, this opens up the possibility of producing a large number of distinct materials using only one type of polymer [Lee07].

The volume fraction of the dispersed phase is only one parameter that affects the morphology. By changing other parameters like the average pore size, the pore size distribution, the pore wall thickness, or the interconnectivity of the pores, the properties of porous polymers can be modified even further. For instance, in buoyancy products like floatation aids, closed-pore porous polymers are required since penetration of water into the material has to be avoided [Mil07]. Open-pore porous polymers, on the other hand, are used as acoustic damping materials for example [Ban11]. However, the interconnectivity, the average pore size, and the pore size distribution are difficult to control when the porous polymer is produced industrially. One method for solving this problem is the use of templates. These consist of a dispersed phase that acts as placeholder for the pores and a continuous phase which is a precursor to the polymer. Since the templates are liquid, their structure is easier to control and manipulate compared to the solid porous polymer. By preserving the generated structure during solidification, the structure of the porous polymer is controlled as well.

If emulsion droplets are used as placeholders in a template, the term "emulsion templating" is used. The continuous phase consists of the monomer(s), the surfactant, and in some cases a 
solvent, while a liquid that is immiscible with the continuous phase serves as the dispersed phase. By monitoring the volume fraction $\Phi_{\text {disp }}$ of the dispersed phase, the density of the porous polymer is adjustable: the higher the amount of the dispersed phase is, the lower is the density. If $\Phi_{\text {disp }}$ is at least $74 \mathrm{vol} \%$, the emulsion is called a high internal phase emulsion (HIPE) [Cam96]. HIPEs were first used to produce porous polymers by Bartl et al. in 1962 [Bar62]. They formulated "reverse emulsions" where water was the dispersed phase, styrene or methyl methacrylate were the two investigated monomers, and a graft copolymer of styrene and polyethylene oxide was used as surfactant. It took another 20 years before a patent was filed for this specific kind of synthesis [Bar85] and the terms HIPE and polyHIPE (polymerized high internal phase emulsion) were introduced although, strictly speaking, the final porous polymer is not an emulsion anymore. Since then, numerous research groups have used this method to prepare porous polymers where the final morphology can be controlled to a certain point [Wil88a, Wil88b, Wil89, Wil90a, Wil90b, Hai91, Cam96, Cam97, Bar00, Cam00, Tai01a, Tai01b, Cam05, Sil05, Kim11, Sil14].

Talha Gokmen et al. first combined HIPEs with microfluidics, which enables the formation of droplets with a uniform size. However, they used this concept to produce monodisperse polymer beads by polymerizing the monomeric droplets in an oil-in-water $(\mathrm{o} / \mathrm{w})$ emulsion [Tal09]. In contrast, Costantini et al. synthesized porous polymers with uniform sized pores by polymerizing the hydrophilic continuous phase of an o/w emulsion formed with microfluidics [Cos14]. Quell et al. used the same method with the hydrophobic monomers styrene and divinylbenzene (DVB) as continuous phase and water as dispersed phase. The polymerization

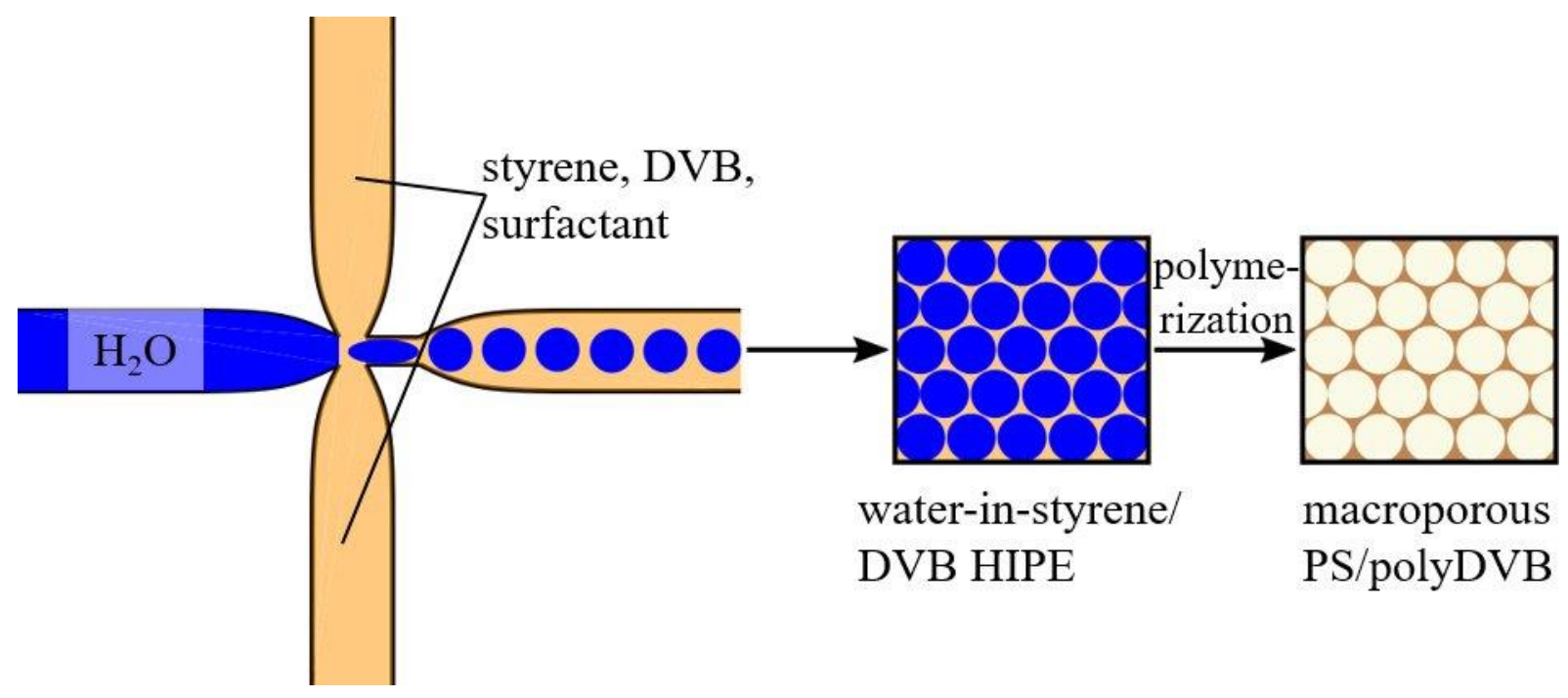

Figure 1.1: Schematic depiction of the synthesis of monodisperse macroporous PS/polyDVB (right) from a monodisperse water-in-styrene/DVB HIPE template (middle) which itself was synthesized with microfluidics (left). 
of this water-in-styrene/DVB HIPE and subsequent drying led to porous polystyrene (PS)/polydivinylbenzene (polyDVB) with a monodisperse pore size [Que15, Que16a, Que16b, Els17, Que17a, Que17b]. The schematic process of producing monodisperse porous PS/polyDVB with monodisperse water-in-styrene/DVB HIPEs is shown in Figure 1.1. By varying the flow rates of both phases in the microfluidic chip, the synthesis of porous PS/polyDVB with pore sizes ranging from $55 \mu \mathrm{m}$ to $80 \mu \mathrm{m}$ was possible. Due to the pore size being larger than $50 \mathrm{~nm}$, the material is classified as macroporous PS/PDVB [Eve72, Bur76]. Furthermore, by changing the type of initiator, both open-pore and closed-pore macroporous PS/polyDVB were obtained. With water-soluble potassium peroxydisulfate (KPS) as initiator, polyhedral and closed pores with granular pore surfaces and layered pore walls are formed (see Figure 1.2 (top)). Note that in the 2D pore cross-sections of Figure 1.2, the polyhedral pores appear as hexagons, while the pores are shaped like rhombic dodecahedrons in 3D.
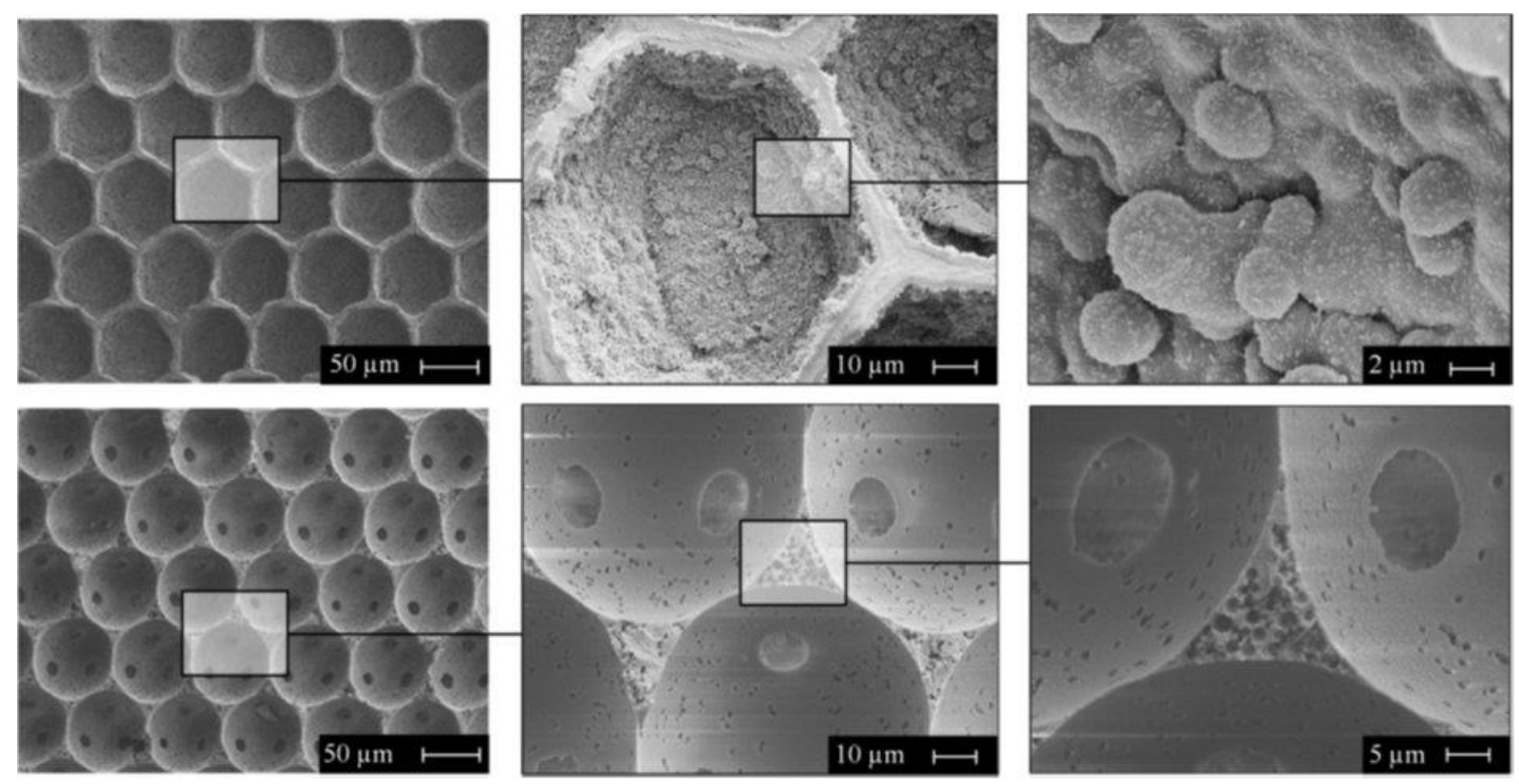

Figure 1.2: Scanning electron microscope (SEM) pictures of macroporous PS/polyDVB obtained from monodisperse water-in-styrene/DVB HIPEs and initiated with (top) KPS and (bottom) AIBN. This Figure was copied from [Que17b]. Reprinted with permission from Quell, A.; Sottmann, T.; Stubenrauch, C.; Diving into the Finestructure of Macroporous Polymer Foams Synthesized via Emulsion Templating: A Phase Diagram Study, Langmuir 2017, 33, 537-542. Copyright (2021) American Chemical Society.

With oil-soluble azobisisobutyronitrile (AIBN) as initiator, on the other hand, spherical and interconnected pores with smooth pore surfaces and porous pore walls are formed (see Figure 1.2 (bottom)). For the samples seen in Figure 1.2, the styrene/DVB mass ratio is 50/50, the surfactant mass fraction $10 \mathrm{wt} . \%$, and the initiator molar concentration $1.28 \mathrm{~mol} \%$. Since the emulsion templates are spherical droplets, the difference observed for the two initiators raises 
the question of how and why the spherical droplets become polyhedral pores when KPS is used. Quell et al. explained this transformation with osmotic transport of DVB [Que17a]. According to Schwachula [Sch75], the polymerization of the crosslinker DVB is faster than that of the monomer styrene, which is why Quell et al. argue that a DVB-rich polymer layer forms at the interface. Assuming that the major part of the monomer styrene only reacts after DVB has polymerized, Quell et al. concluded that layered pore walls with two polyDVB-rich outer layers and a PS-rich inner layer form. Furthermore, a concentration gradient of DVB between the Plateau borders (initial DVB concentration) and the films (DVB depleted) is believed to be the reason for the change from a spherical to a polyhedral pore shape. This gradient causes osmotic transport of DVB molecules from the Plateau borders into the films. As a result, the size of the Plateau borders is reduced and the thickness of the films is increased. Thus, the shape of the interface changes from spherical to polyhedral. Furthermore, Quell et al. showed that quite uniform pore wall thicknesses are built. Note that the osmotic transport has to work against the desire of the droplets to minimize their surface since the surface area of a rhombic dodecahedron is $\sim 10 \%$ larger than the surface area of a sphere [Que16b, Que17a].

\subsection{Task Description}

Although the experimental results obtained by Quell et al. [Que17a] support the idea of an osmotic transport of DVB as the reason for the transformation from spherical droplets to polyhedral pores, resounding proof for this hypothesis is still lacking. Thus, the main focus of this $\mathrm{PhD}$ thesis is put on either proving or disproving this hypothesis. To achieve this, logical deductions are drawn about what ought to happen to the morphology of monodisperse macroporous PS/polyDVB when the concentration of a component is changed systematically. (1) When the styrene/DVB mass ratio is changed from 50/50 towards pure styrene, the extent of osmotic transport of DVB is expected to decrease. Thus, the shape of the pores ought to become more spherical. Additionally, since less DVB is present in the continuous phase, the thickness of the supposedly polyDVB-rich outer layers is expected to decrease. (2) When the KPS concentration is increased, the polymerization rate ought to increase as well. Thus, osmotic transport of DVB has less time to occur and the pores ought to become more spherical as well. In both cases, analogous experiments are conducted with AIBN as initiator for the sake of comparison.

If the hypothesis of osmotic transport of DVB is confirmed, the large number of new experimental results may allow a quantitative description of the mechanism. On the other hand, 
if the experiments reveal that osmotic transport of DVB is not responsible for the transformation of spherical droplets to polyhedral pores, this hypothesis was to be dismissed. Consequently, a new hypothesis would have to be developed by conducting further experiments. The new hypothesis would have to comply with both old and new experimental results.

Finally, the mechanical properties of monodisperse macroporous PS/polyDVB are examined. Focus is put on how the type and amount of initiator, the styrene/DVB mass ratio, and the amount of surfactant influence the mechanical properties. The goal is to obtain structureproperty-relationships. Moreover, both monodisperse and polydisperse macroporous PS/polyDVB are investigated to see if their mechanical properties are different. 
$-6-$ 


\section{Theoretical Background}

\subsection{Emulsions}

If two pure liquids are brought in contact, there are three possibilities for what can happen: a chemical reaction, mixing, and separation. In the latter case, the mixtures are typically composed of a hydrophilic liquid, in most cases water, and a hydrophobic liquid which is often called "oil". To enable the mixing of immiscible liquids, a third component is required which is at least partially soluble in either liquid. Such molecules typically consist of a hydrophilic and a hydrophobic part. In a ternary mixture, this causes an accumulation of this molecule at the interface between water and oil, thereby reducing the interfacial tension between them. The same is true when the hydrophobic liquid is substituted by a gas which transforms the interface into a surface. This type of molecule is therefore called "surfactant" (surface active agent) or "amphiphile". Mixtures of water and oil stabilized with surfactants are called "emulsions", while the surfactant is called "emulsifier". Emulsions have found their way into modern life via cosmetics like creams, shampoos, make-up, or perfume as well as into industrial processes. Typical emulsions either consist of water droplets dispersed in a continuous oil phase (w/oemulsions, see Figure 2.1 (left)) or of oil droplets dispersed in a continuous water phase (o/wemulsions see Figure 2.1 (right)).
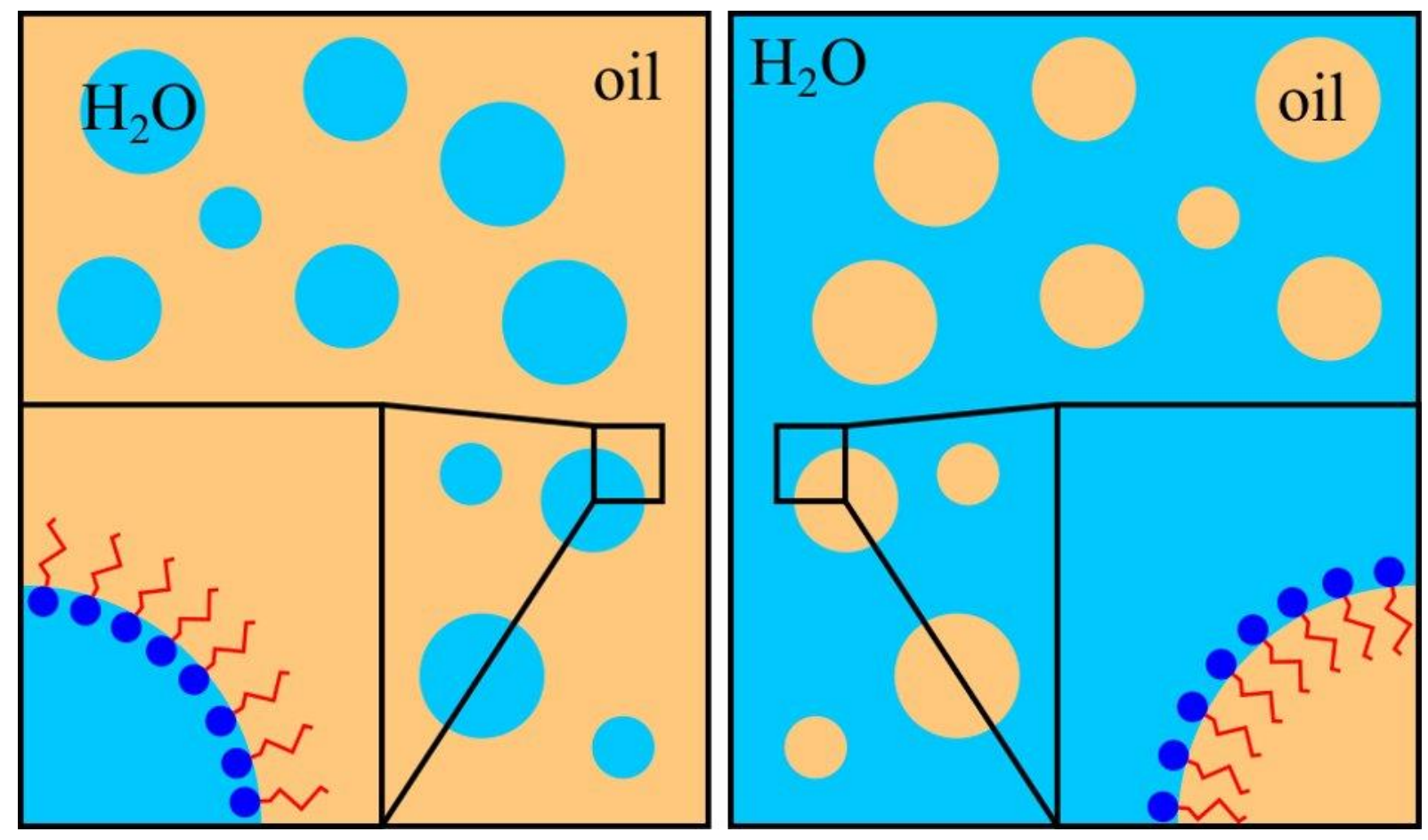

Figure 2.1: Schematic structure of (left) a water-in-oil emulsion and (right) an oil-in-water emulsion. 
More complex systems like o/w/o- or w/o/w-emulsions also exist but less common. The type of emulsion depends on the type of surfactant, the temperature, the concentration of electrolytes in the water phase, the volume fractions, and the concentration of the surfactant. To test which type of emulsion has formed, two easy experiments can be conducted. One the one hand, if low amounts of salt are added and the conductivity is measured, o/w-emulsions show a high conductivity while the value is close to zero in the case of w/o-emulsions. On the other hand, a dye which is only soluble in one phase can be added. The color can only be seen if the dye is dissolved in the continuous phase [Bin98, Eva99, Lyk05, Ros12].

In general, most emulsions appear white since the droplets of the dispersed phase have a diameter between $200 \mathrm{~nm}$ and $100 \mu \mathrm{m}$, thus scattering visible light. However, emulsions can also be transparent, namely if the dispersed droplets are diluted enough or the if their diameter is smaller than $200 \mathrm{~nm}$. In this case, the Tyndall effect [Whi20] can be observed. When a ray of light is shone through a sample, light scattering in the liquid is visible if particles with a diameter in the micrometer range are present which of course is true for emulsions. In homogeneous liquids, in contrast, no ray of light is observable [Sch05].

\section{Influence of Interfacial Tension on Emulsions}

The surfactant molecules between the dispersed and the continuous phase form a skin-like layer around the dispersed droplets which enables a kinetic stabilization of the interface. No matter how well an emulsion might be stabilized kinetically, its ultimate fate is always phase separation. The reason behind this lays in the change of the Gibbs free energy $G$ which in the case of emulsions holds

$$
G=H-T \cdot S_{\mathrm{td}}+\gamma \cdot A_{\text {interface }}
$$

In equation (2.1), $H$ represents the enthalpy, $T$ the temperature, $S_{\mathrm{td}}$ the thermodynamic entropy, $\gamma$ the interfacial tension, and $A_{\text {interface }}$ the interfacial area. Note that thermodynamic systems always try to minimize $G$ [Eva99]. Since the difference in enthalpy is zero between an emulsion and two separated phases, only the second and third term are important for the stability of emulsions. On the one hand, by emulsifying two originally separated phases, $S$ increases which

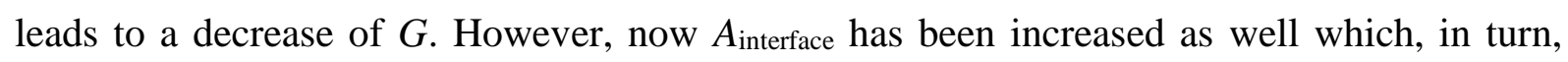
increases $G$. Thus, the deciding factor is how far the surfactant can reduce the value of $\gamma$. This decrease is by far not enough in the case of emulsions (typically $\gamma \approx 30 \mathrm{mN} \mathrm{m}^{-1}$ ), thus making 
them overall thermodynamically unstable [Lyk05]. In microemulsions, in contrast, the interfacial tension is reduced to $\gamma \approx 1 \mathrm{mN} \mathrm{m}^{-1}$ or lower. For example, the system $\mathrm{H} 2 \mathrm{O}$ - linear alkylbenzene sulphonate (LAS) - decane with $0.1 \mathrm{~g} \mathrm{~L}^{-1}$ LAS has an interfacial tension of $\gamma \approx$ $0.5 \mathrm{mN} \mathrm{m}^{-1}$ [Stu09]. In this case, the increase in $S$ matters and the system becomes thermodynamically stable. Furthermore, microemulsions appear transparent because droplets with typical diameters between $10 \mathrm{~nm}$ and $100 \mathrm{~nm}$ form and are thus not able to scatter light [Stu09].

Another point where $\gamma$ matters is the shape of emulsion droplets. For a given volume, a sphere has the lowest surface area and since a minimized surface area is required energetically, the droplets in emulsions are spherical. The diameter of the droplets is determined by the interfacial tension and the pressure difference between the inner and the outer liquid. This pressure difference tries to increase the diameter of the droplets, while the surface tension works against this effort. The two counteracting forces lead to an equilibrium droplet diameter which can be described with the Young-Laplace equation. It holds

$$
\Delta p=2 \cdot \gamma \cdot \kappa
$$

where $\Delta p$ represents the pressure difference between the inner and the outer liquid and $\kappa$ the mean curvature of the droplets. It holds

$$
\kappa=\frac{1}{2} \cdot\left(\frac{1}{r_{1}}+\frac{1}{r_{2}}\right)
$$

where $r_{1}$ and $r_{2}$ are the two radii of an ellipsoidal body. In the case of spheres, the two radii are equal ( $\left.r_{\text {sphere }}\right)$ which simplifies the curvature to the reciprocal radius and transforms equation (2.2) to [Sch05]

$$
\Delta p=\frac{2 \gamma}{r_{\text {sphere }}}
$$

\section{Disintegrating Mechanisms}

Since emulsions are only kinetically stabilized but not thermodynamically stable, they will eventually disintegrate. There are three different routes for how that can occur, namely sedimentation (or creaming), coalescence, and Ostwald ripening (see Figure 2.2). 


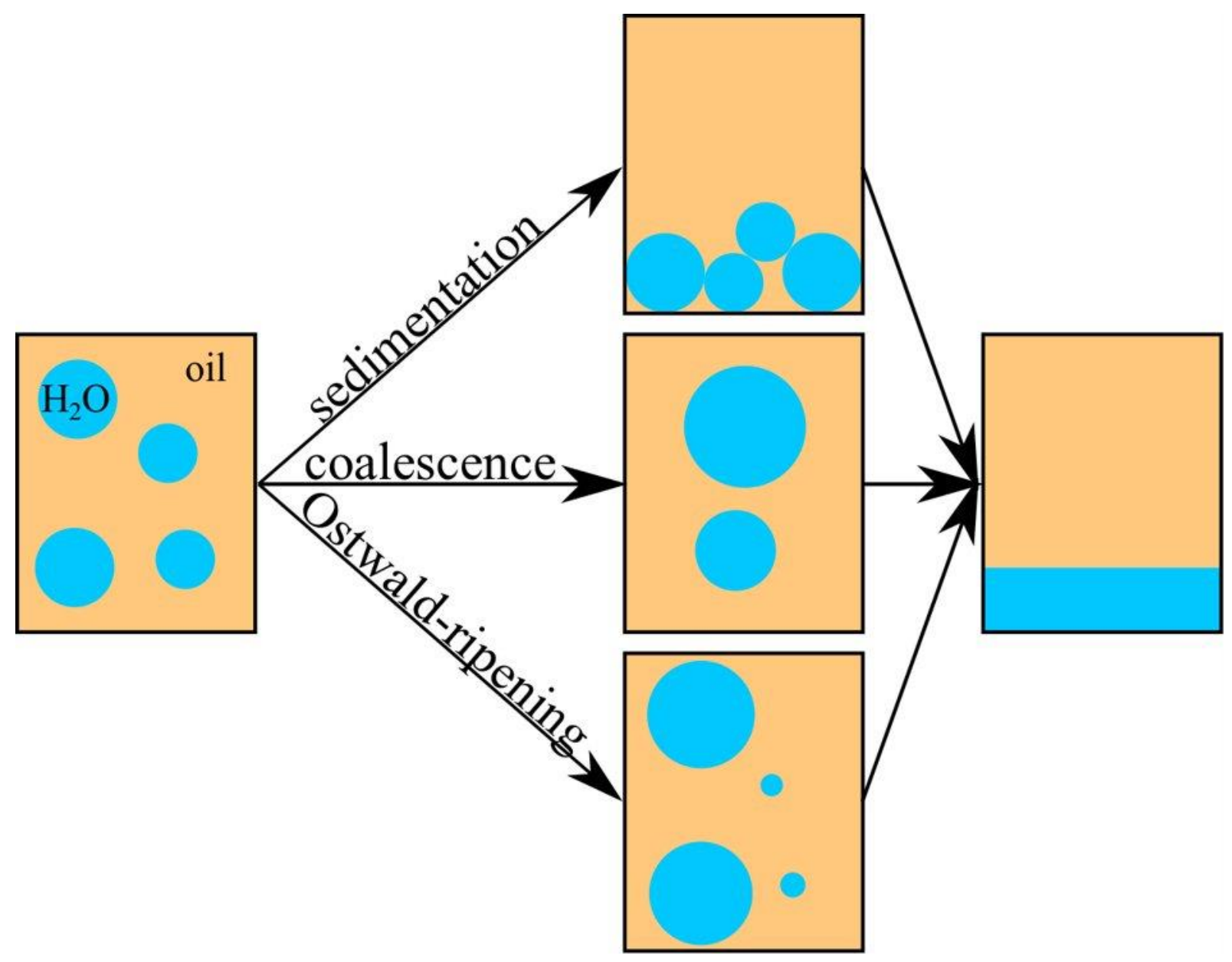

Figure 2.2: Schematic depictions of the three disintegrating mechanisms (top) sedimentation, (middle) coalescence, and (bottom) Ostwald-ripening for a $\mathrm{o} / \mathrm{w}$ emulsion. Note that in $\mathrm{o} / \mathrm{w}$ emulsions, sedimentation is usually replaced by creaming.

One way how emulsions can decompose is by an accumulation of droplets. If the dispersed phase has a higher density than the continuous phase, which is typically the case for w/o-emulsions, the droplets sediment towards the bottom due to gravity (see Figure 2.2 (top)). On the other hand, if the dispersed phase is less dense than the continuous phase, which usually applies for o/w-emulsions, the opposite happens. The droplets than cream towards the top of the emulsion. Eventually, these processes lead to a phase separation if the sedimented or creamed droplets merge. An approach to at least slow down sedimentation or creaming is the use of two liquids with similar densities or to artificially modify the densities via additives [Bin98, Eva99].

Another route of decomposing is the coalescence of droplets. This can happen when they are brought close enough together through Brownian motion or flocculation. Note that flocculation can serve as its own disintegrating route if the droplets aggregate irreversible. Due to gravity, this would cause sedimentation or creaming at some point. Coalescence, on the other hand, is 
a process where two droplets fuse together to form one bigger droplet (see Figure 2.2 (middle)). This can only occur if the surfactant film is not stable enough to prevent this, i.e. if the surfactant concentration is not high enough. The merging of two droplets is energetically favored since the Laplace pressure of the larger droplet is smaller (see equation (2.4)). The coalescence of more and more droplets eventually leads to phase separation. A way to avert coalescence is using a sufficient concentration of surfactant. The steric repulsion of non-ionic surfactants or the electrostatic repulsion of ionic surfactants, respectively, pushes the droplets apart if they come to close together [Bin98, Eva99, Sch05].

Finally, an emulsion can decompose via Ostwald ripening. Named after its discoverer W. Ostwald [Ost00], this effect describes a process where bigger droplets increase their size at the expense of smaller ones (see Figure 2.2 (bottom)). Note that Ostwald ripening is not exclusive to emulsions but also occurs in foams. The underlying principle is the Young-Laplace equation (see equation (2.4)). For smaller droplets, the pressure difference between inner and outer phase is higher than for bigger droplets because $\kappa$ is larger for smaller droplets. Since the dispersed liquid has a finite solubility in the continuous one, single molecules can inter-diffuse between droplets. Additionally, their concentration close to a droplet is dependent on the diameter of the droplet, being higher in the case of smaller droplets. As a result, a concentration gradient between the outside of small droplets - where the concentration of solubilized molecules is high - and the outside of large droplets - where the concentration of solubilized molecules is low forms. Therefore, molecules of the dispersed phase will net-diffuse from the small droplets through the continuous phase into large droplets. Eventually, this leads to a disappearance of smaller droplets, while the bigger droplets grow larger and larger, finally also resulting in phase separation. The easiest way to avoid Ostwald ripening is creating a monodisperse emulsion, i.e. creating droplets that all have the same Laplace pressure. The droplets then retain the same size for a long time. This can be achieved by preparing the emulsion via microfluidics (see Chapter 2.2) or by preparing a solution already assembled from all three components (water, oil, and amphiphile) and rapidly adding more oil or water. Once the system crosses into the two-phase region, uniform sized droplets form via nucleation. The ouzo effect observed in many alcoholic beverages derived from plants containing essential oils is a famous practical example for this method [Vit03]. Another route to prevent Ostwald ripening is to dissolve an additive in the dispersed phase which is completely insoluble in the continuous phase, e.g. for w/o emulsions a salt like sodium chloride $\mathrm{NaCl}$. In the beginning, the concentration of $\mathrm{NaCl}$ is equal in all droplets. However, if a net diffusion of molecules of the dispersed phase into the bigger droplets occurs, a reverse chemical potential arises as well. Therefore, an osmotic transport forces a 
back-diffusion into the smaller droplets, thus counterbalancing Ostwald ripening. In the case of $\mathrm{o} / \mathrm{w}-\mathrm{emulsions}$, the same effect can be achieved if long hydrocarbons like hexadecane are used [Bin98, Sch05].

\section{Polydispersity, Volume Fraction, Packing, and Ordering in Emulsions}

The polydispersity and the volume fractions of the dispersed and the continuous phase, respectively, also have an influence on the structure and stability of emulsions. The polydispersity index $(P D I)$ describes how large the droplet size distribution in an emulsion is. With $d_{\mathrm{sd}}$ as the standard deviation of the measured droplet diameters and $d_{\text {average }}$ as the average droplet diameter, it holds [Cla16]

$$
P D I=100 \% \cdot \frac{d_{s d}}{d_{\text {average }}} .
$$

Typical emulsions have a PDI of around 50\% and are therefore polydisperse. Ideal monodisperse emulsions, on the other hand, would have an PDI of 0. However, because "true" monodispersity is virtually impossible, the term monodispersity is typically used when the PDI is equal to or smaller than 5\% [Mae13].

Furthermore, the volume fraction of the dispersed phase $\left(\Phi_{\text {disp }}\right)$ is used to classify different types of emulsions. It holds

$$
\Phi_{\text {disp }}=\frac{V_{\text {disp }}}{V_{\text {disp }}+V_{\text {cont }}}=\frac{V_{\text {disp }}}{V_{\text {total }}}
$$

with $V_{\text {disp }}$ denoting the volume of the dispersed phase, $V_{\text {cont }}$ denoting the volume of the continuous phase, and $V_{\text {total }}$ denoting the total volume. If $\Phi_{\text {disp }} \approx 25 \%$, the emulsion is called low internal phase emulsion (LIPE, see Figure 2.3 (first column)). For $30 \%<\Phi_{\mathrm{disp}}<70 \%$ and for $\Phi_{\text {disp }}>74 \%$, the terms medium internal phase emulsion (MIPE see Figure 2.3 (second column)) and high internal phase emulsion (HIPE, see Figure 2.3 (third column)) are used. 


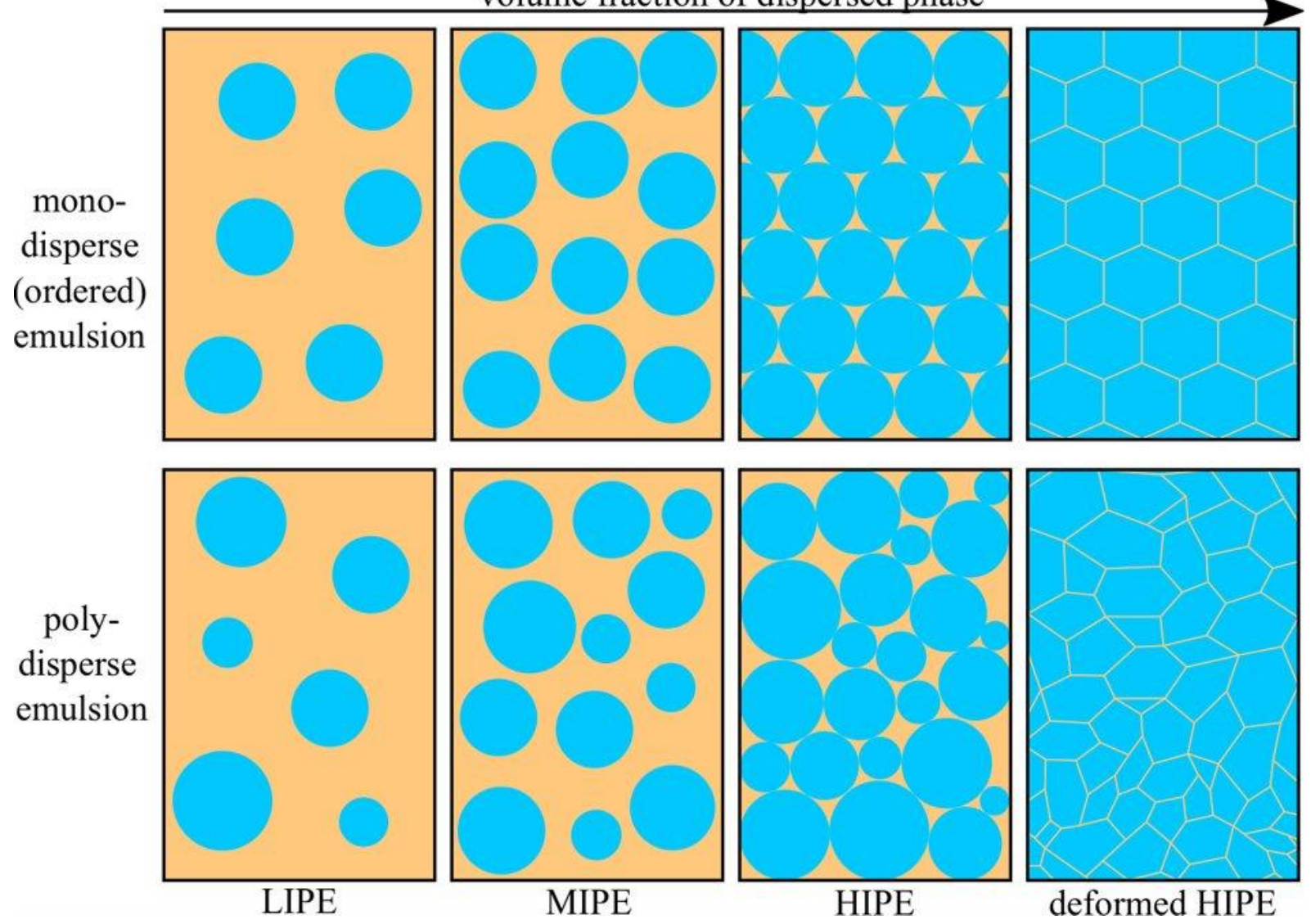

Figure 2.3: Schematic depiction of (top) monodisperse and (bottom) polydisperse emulsions with increasing volume fractions of the dispersed phase (from left to right).

In HIPEs, due to gravity and the high volume fraction of the dispersed phase, the droplets are in contact. A further distinction can be made concerning their packing fraction and their ordering. The first parameter describes how "well" the droplets are or can be packed, i.e. how much continuous phase can be removed between the droplets without deforming them. Packing problems are very common in Mathematics and solving them is far from trivial. Monodisperse droplets have a maximum packing fraction of $74 \%$ which is the maximum packing fraction of any set of spheres with a uniform diameter. This phenomenon has been known since 1611 when it was described by J. Kepler and is called the Kepler conjecture. However, it took about another 400 years before it was finally proven mathematically [Hal06].

If the emulsion is polydisperse, different packing fractions are possible. If the PDI was high and the droplets were packed manually, the packing fraction could exceed $95 \%$. The reason for this is that smaller droplets can be packed into the free spaces between the bigger droplets (see Figure 2.3 (bottom, third column)). However, this process does not occur automatically which means that extra force needs to be applied. In contrast, if the PDI was rather small, the packing fraction can be approximated to $64 \%$, which correlates to the close packing limit of randomly 
packed monodisperse spheres [Ber60]. Another term that can be used instead of 'randomly packed' is disordered. If the droplets are ordered, however, they reach their maximum packing fraction at $74 \%$. Since the dispersed phase is not composed of hard spheres but liquid droplets, the packing fraction can exceed $64 \%$ or $74 \%$, respectively. In that case, the droplets need to be deformed and start to become polyhedral (see Figure 2.3 (fourth column)).

\subsection{Microfluidics}

Describing " $[\mathrm{t}]$ he science and technology of systems that process or manipulate small $\left(10^{-9}-\right.$ $10^{-18}$ liters) amounts of fluids, using channels with dimensions of tens to hundreds of micrometers" [Whi06], systematic studies of microfluidics date back as far as the 1970s. At that time, microfluidic devices were fabricated with micromechanics technology and were employed in research on gas chromatography and ink jet printing [Gra93, Tab05]. However, it was not until the early 1990s that progress in the field of microfluidics really picked up [Jon06, Whi06]. In essence, the applications of microfluidics can be divided into (a) the synthesis of chemicals and materials and (b) the analysis of mostly biological samples. All types of applications avail themselves of the numerous advantages of this technique. Among these are the low consumption of chemicals and samples, the enhanced separation and detection of samples with remaining high resolution and sensitivity, the low cost, the short time for analysis, and the small footprint on analytical devices [Man92, Bee02, Whi06, Zha11, Zha16]. Another advantage of microfluidic devices is the fact that the fluids show laminar ("sheet-like") flow, which can be exploited for specific applications and will be discussed to a greater extent later. Laminar flow means that the hypothetical sheets inside a fluid flow parallelly and do not automatically mix with each other.

Commonly, polydimethylsiloxane (PDMS) is used as the base material from which the microfluidic devices are manufactured by means of soft lithography techniques. PDMS has several advantages, namely it is a moldable elastomeric polymer, it is inexpensive, and it is optically transparent. Nevertheless, glass, steel, silicone, and thiolene can also be used when more chemical or mechanical resistance is required. These materials do not deform or swell when they come into contact with strong organic solvents or when they are exposed to higher temperatures [Whi06, Chr07, Teh08]. Sometimes, it is necessary to change the wettability of the microfluidic chip, i.e. to render its surface hydrophobic or hydrophilic. In order to transform a hydrophilic surface into a hydrophobic one, it can be silanized or siliconized. On the other hand, to transform a hydrophobic surface into a hydrophilic one, it can be exposed to oxygen 
plasma, polyvinyl alcohol (PVA), or polyethylene glycol (PEG). Another way of changing the surface wettability is the use of surfactants which absorb at the surface, thus rendering the chip hydrophobic or hydrophilic [Chr07, Teh08].

A particular subtopic of microfluidics is droplet-based microfluidics where two phases are used instead of one. They are either partially miscible or immiscible [Zha11]. When the two phases meet at the so-called "junction", monodisperse emulsion droplets are formed. In general, the fluids are pushed through the microfluidic device by means of pressure pumps or syringe pumps. At this point, it is necessary to take a closer look at the way the droplets are generated inside the microfluidic device. By and large, the droplet generation is determined by (a) the channel geometry and (b) the forces acting upon the fluids as well as upon the arising interface. The ratio of two of these forces can be expressed in the form of dimensionless numbers, which, along with other parameters, help to subdivide the droplet generation into different regimes.

\section{Channel Geometry}

Most of the used channel designs can be classified into cross-flow geometry, co-flow geometry, and flow-focusing geometry, the last of which also being referred to as elongated flow geometry [Chr07, Bar10, Zha11, Zha16]. Further arrangements dealing with step emulsification, microchannel emulsification, and membrane emulsification are less common [Zhu16] and will not be discussed here. A visual representation of cross-flow, co-flow, and flow-focusing geometry is given in Figure 2.4. In cross-flow geometry, the two phases meet at an angle $\theta$ of between $0^{\circ}-180^{\circ}$ (see Figure 2.4 (top left)). In most cases, $\theta=90^{\circ}$ and this particular arrangement is called "T-junction" (see Figure 2.4 (top right)). The dispersed phase and the continuous phase ought to have similar flow rates in order to enable droplet generation. If the flow rate of the dispersed phase is much lower than that of the continuous phase, the former will not enter the downstream channel and hence, no droplets will form. If, on the other hand, the flow rate of the continuous phase is too low compared to that of the dispersed phase, both of them will stream uninterruptedly into the downstream channel and hence, droplets will not form either. If the ratio between the two phases is set to an appropriate and constant value, uniform-sized droplets will form periodically. This was first achieved by Thorsen et al. in 2001 [Tho01], who generated an w/o emulsion with a PDI as low as $2 \%$. Since then, the cross-flow geometry and especially the T-junction have been widely used due to their easy handling [Chr07, Zhu16]. 


\section{cross-flow geometry}

co-flow geometry

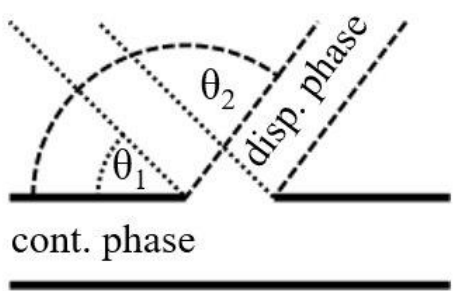

general

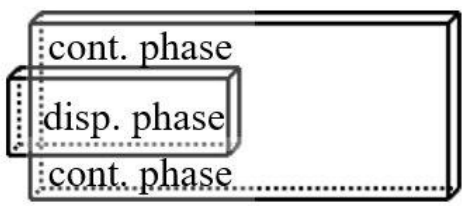

2D-planar

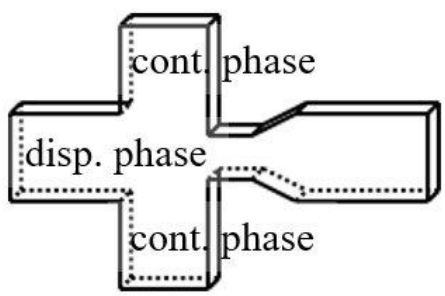

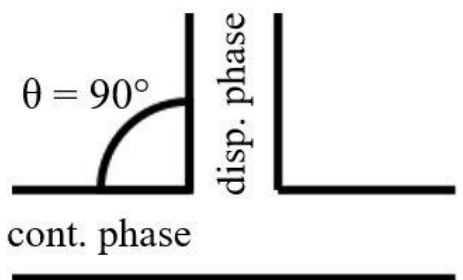

T-junction

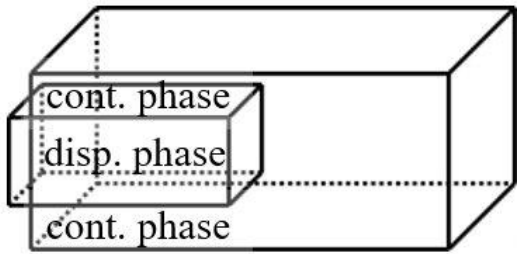

3D-coaxial

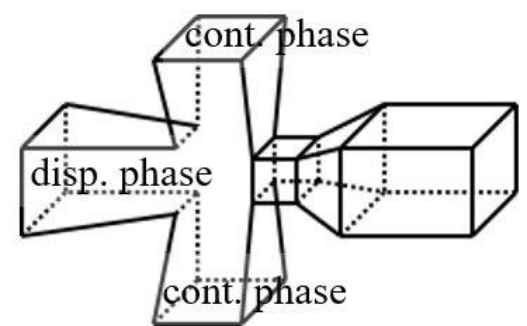

Figure 2.4: Schematic depiction of the general cross-flow geometry (top left), the T-junction geometry (top right), the 2D-planar co-flow geometry (middle left), the 3D-coaxial co-flow geometry (middle right), the 2D-planar flow-focusing geometry (bottom left), and the 3Dcoaxial flow-focusing geometry (bottom right).

As its name already suggests, in the co-flow geometry the two phases flow coexistingly (parallelly) with the channel of the dispersed phase being surrounded by the larger channel of the continuous phase (see Figure 2.4 (middle row)). Typically, the size of the generated droplets is larger than the size of the channel of the dispersed phase. With a co-flow geometry, the first monodisperse droplets were produced by Umbanhower et al. [Umb00], who were able to minimize the $P D I$ in their o/w emulsion to $3 \%$. When focusing on the "depth" of the channels, co-flow geometry can either be two dimensional (2D)-planar or three dimensional (3D)-coaxial (see Figure 2.4 (middle left and middle right)) [Chr07, Zhu16].

Finally, in flow-focusing / elongated flow geometry both phases are pushed through a narrow constriction (see Figure 2.4, bottom row). Moreover, the dispersed phase is flanked by two streams of the continuous phase with a velocity flow field of the continuous phase focusing the dispersed phase. At the highest point of shear, the result is a consistent breakup of the dispersed phase into uniform-sized droplets. In general, this geometry offers an even more controlled droplet break-up than the other two that were discussed previously. As is case for co-flow 
geometry, the channels can either be 2D-planar or 3D-coaxial (see Figure 2.4 (bottom left and bottom right)). In 2001, Gañàn-Calvo et al. [Gan01] were the first to use this geometry for producing gas-in-water $(\mathrm{g} / \mathrm{w})$ bubbles. One year later, Anna et al. [Ann02] employed the same arrangement for generating monodisperse w/o emulsion droplets [Chr07, Teh08, Zhu16].

\section{Forces, Dimensionless Numbers, and Droplet Break-Up}

In essence, there are four forces ${ }^{1}$ that act upon the fluids inside a microfluidic device. They all depend on the characteristic length scale $L$ of the microfluidic device - most often simply the channel thickness - and/or the velocity $v_{\mathrm{i}}$ of the fluid. The index ' $i$ ' thereby denotes the type of phase that is examined with ' $c$ ' indicating the continuous phase and ' $d$ ' indicating the dispersed phase. The first force acting upon a fluid is the inertial force $f_{\mathrm{i}}$ which holds

$$
f_{\mathrm{i}}=\rho_{\mathrm{i}} \cdot v_{\mathrm{i}}^{2},
$$

with $\rho_{\mathrm{i}}$ being the density of the fluid. This force stems from the pressure/syringe pump that sets the liquid in motion inside the microfluidic device. At the junction where the two phases meet, this force squeezes both of them downstream towards the channel outlet. The second force that has the same orientation is the viscous force $f_{\mathrm{v}}$ which holds

$$
f_{\mathrm{v}}=\frac{\eta_{\mathrm{i}} \cdot v_{\mathrm{i}}}{L}
$$

with $\eta_{i}$ being the dynamic viscosity. As its name presumes, this force is caused by the viscosity of the fluid which itself stems from molecular diffusion and intermolecular interactions. The third force that becomes especially important once the two immiscible phases get into contact with each other is the capillary pressure $f_{\mathrm{c}}$ which holds

$$
f_{\mathrm{c}}=\frac{\gamma}{L}
$$

with $\gamma$ being the interfacial tension between the two phases. If only one phase is present, $\gamma$ denotes the surface tension of the fluid. Finally, gravity has an influence on the fluids inside a microfluidic device as well. Its force $f_{\mathrm{g}}$ holds

$$
f_{\mathrm{g}}=\rho_{\mathrm{i}} \cdot g \cdot L
$$

\footnotetext{
${ }^{1}$ Note that the four forces only nominally carry the name "force", but are not forces in terms of physics.
} 
with $g$ being the gravitational constant of the earth [Zhu16]. In order to determine which type of flow is present inside a microfluidic channel - laminar or turbulent - the so-called Reynolds number $R e$ is calculated. This dimensionless number is given by the ratio between the inertial force $f_{\mathrm{i}}$ and the viscous force $f_{\mathrm{v}}$ and thus,

$$
R e=\frac{f_{i}}{f_{\mathrm{v}}}=\frac{\rho_{\mathrm{i}} \cdot v_{\mathrm{i}}^{2}}{\eta_{\mathrm{i}} \cdot v_{\mathrm{i}} / L}=\frac{\rho_{\mathrm{i}} \cdot v_{\mathrm{i}} \cdot L}{\eta_{\mathrm{i}}}
$$

[Bee02, Gar05, Zhu16]. If the viscous force $f_{\mathrm{v}}$ greatly exceeds the inertial force $f_{\mathrm{i}}, R e$ is small and the flow is laminar. On the other hand, if the inertial force $f_{i}$ greatly exceeds the viscous force $f_{\mathrm{v}}, R e$ is large and the flow is turbulent. The boundary between laminar and turbulent flow is typically put at $R e=2300$ [Bee02, Zha16]. However, in microfluidic channels $R e$ is usually between $10^{-6}$ and $10^{1}$ [Zhu16] and therefore, the flow is always laminar in microfluidic channels. Fortunately, the famous Navier-Stokes-equation for incompressible fluids can be solved linearly for laminar flow. For turbulent flows, solving the Navier-Stokes-equation requires non-linear differential equations [Whi01, Squ05].

When droplets are generated, an interface between the two phases is formed. Thus, the force of the capillary pressure $f_{\mathrm{c}}$, which contains the interfacial tension $\gamma$ becomes essential. The energy for developing the interface is provided by the pressure / syringe pumps that set the fluid in motion. All the other three forces in relation to the force of the capillary pressure $f_{\mathrm{c}}$ can be expressed as dimensionless numbers. The Bond number Bo gives the ratio of the gravitational force $f_{\mathrm{g}}$ to the force of the capillary pressure $f_{\mathrm{c}}$ and thus,

$$
B o=\frac{f_{\mathrm{g}}}{f_{\mathrm{c}}}=\frac{\Delta \rho \cdot g \cdot L}{\gamma / L}=\frac{\Delta \rho \cdot g \cdot L^{2}}{\gamma} .
$$

Here, $\Delta \rho$ is the difference in density between the two phases. When emulsion droplets are formed inside a microfluidic device, both $L$ and $\Delta \rho$ are small, which, in turn, decreases $B o$ to miniscule values. Consequently, the influence of gravity can be neglected for droplet generation in a microfluidic device [Tab05, Chr07, Zha11, Zhu16]. Note that for bubble generation, $\Delta \rho$ has a higher value since the dispersed phase is a gas, which also increases $B o$ to some extent. Here, gravity can have an influence on bubble generation [Chr07].

As already discussed for the Reynolds number Re, the inertial force $f_{\mathrm{i}}$ can usually be disregarded on the microfluidic scale. The ratio of this force to the force of the capillary pressure $f_{\mathrm{c}}$ is expressed by the Weber number We. Therefore, this dimensionless number is given as 


$$
W e=\frac{f_{\mathrm{i}}}{f_{\mathrm{c}}}=\frac{\rho_{\mathrm{i}} \cdot v_{\mathrm{i}}^{2}}{\gamma / L}=\frac{\rho_{\mathrm{i}} \cdot v_{\mathrm{i}}^{2} \cdot L}{\gamma} .
$$

As the Reynolds number Re before, We has small values when droplets are generated in a microfluidic device and is therefore not of high relevance [Chr07, Zha11, Zhu16].

The most important dimensionless number in microfluidics is the Capillary number Ca which expresses the ratio between the two dominating forces on the microfluidic scale, $f_{\mathrm{v}}$ and $f_{\mathrm{c}}$. It holds

$$
C a=\frac{f_{\mathrm{v}}}{f_{\mathrm{c}}}=\frac{\eta_{\mathrm{i}} \cdot v_{\mathrm{i}} / L}{\gamma / L}=\frac{\eta_{\mathrm{i}} \cdot v_{\mathrm{i}}}{\gamma} .
$$

Consequently, $\mathrm{Ca}$ is independent of the length scale $\mathrm{L}$. In microfluidic droplet generation, $\mathrm{Ca}$ typically takes up values between $10^{-3}$ and $10^{1}$ [Zhu16] and helps to differentiate between three droplet generation regimes [Squ05, Chr07, Teh08, Zhu16]. These will be discussed thoroughly in the upcoming paragraphs.

Two further dimensionless numbers that are sometimes used and do not depend on the four previously introduced forces are the viscosity ratio $\lambda$ and the flow ratio $\varphi$. They hold

$$
\lambda=\frac{\eta_{d}}{\eta_{\mathrm{c}}}
$$

and

$$
\varphi=\frac{Q_{\mathrm{d}}}{Q_{\mathrm{c}}}
$$

with $\eta_{d}$ and $\eta_{c}$ representing the viscosities of the dispersed and the continuous phase and $Q_{d}$ and $\mathrm{Q}_{\mathrm{c}}$ representing the flow rates of the dispersed and the continuous phase, respectively [Chr07, Teh08, Zhu16].

Droplet generation in microfluidic devices can be divided into three major regimes. These are squeezing, dripping, and jetting and are depicted in Figure 2.5. Note that since the geometry of the microfluidic device in this $\mathrm{PhD}$ thesis was flow-focusing geometry, the three regimes accessible with this particular geometry are described. Nevertheless, the concepts are very similar in cross-flow and co-flow geometry. Two further, less common regimes are tipstreaming and tip-multibreaking. These are modifications of the jetting regime and will not be 


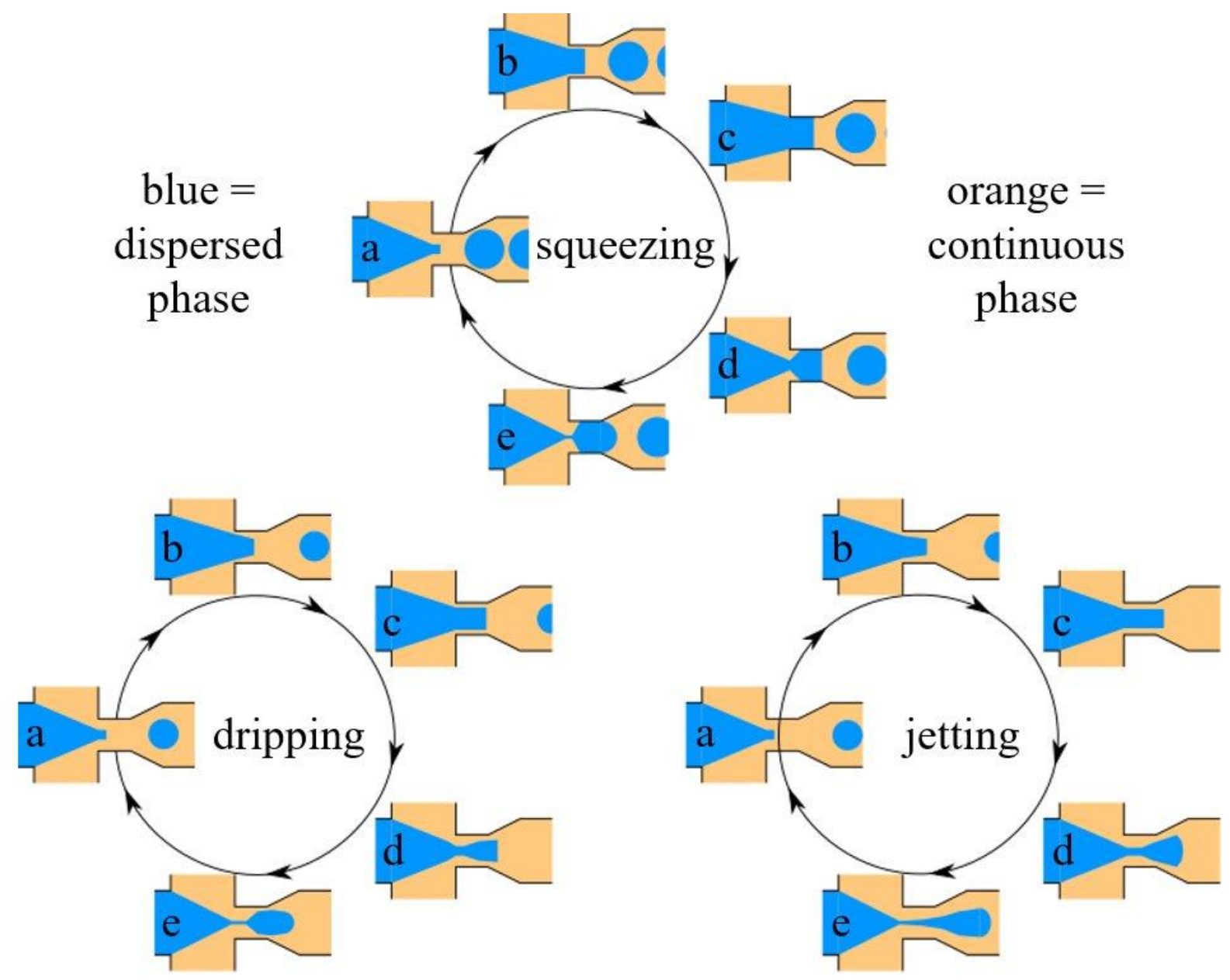

Figure 2.5: Schematic depiction of the generation of one droplet in the squeezing regime (top), in the dripping regime (bottom left), and the jetting regime (bottom right).

discussed here. As already mentioned, the Capillary number $\mathrm{Ca}$ can be used to define the boundaries between the three regimes. In general, the viscous force $f_{\mathrm{v}}$ and to smaller extend also the inertial force $f_{\mathrm{i}}$ try to deform the interface in order to continue flowing downstream, while the capillary pressure tries to minimize the interfacial area. Since $f_{\mathrm{i}}$ can be neglected, the Capillary number $\mathrm{Ca}$ is a good indicator for distinguishing between the three regimes and is therefore conventionally used. When the Capillary number of the continuous phase $C a_{\mathrm{c}}$ is smaller than $10^{-2}$, droplet generation occurs in the squeezing regime. When $C a_{\mathrm{c}}$ is between $10^{-2}-$ $10^{\circ}$, droplet generation occurs in the dripping regime. Finally, when $C a_{\mathrm{c}}$ is larger than $10^{\circ}$, droplet generation occurs in the jetting regime [Ann16, Zhu16]. Together, they are all part of the larger concept of passive droplet generation where droplet break-up is only determined by the flow rates. In contrast, in active droplet generation auxiliary energy is put into the system. For instance, electrical, magnetical, or centrifugal fields or optical stimuli assist the destabilization of the interface which, in turn, facilitates droplet breakup. Another technique of active droplet generation is altering the intrinsic properties of the fluids. Among these intrinsic 
properties are the velocity, the viscosity, the interfacial tension, the channel wettability, and the fluid density [Zhu16]. However, in this $\mathrm{PhD}$ thesis only the interfacial tension was influenced by adding a surfactant and hence, only this way of active droplet generation will be discussed to a greater extend later.

Coming back to the squeezing, dripping, and jetting regimes, the first one is different from the latter two in that its droplet breakup is not determined by capillary instabilities. In contrast, the confinement of the channel is dominating here and droplet breakup is governed by a quasi-static mechanism. In the beginning, the dispersed phase starts to fill up the downstream channel (cross-flow and co-flow geometry) or the constriction (flow-focusing geometry), respectively (see Figure 2.5 (top a, b, and c)). Thereby, the available volume for the continuous phase to continue flowing downstream becomes increasingly limited. This, in turn, creates a pressure gradient of the continuous phase between the downstream channel (lower pressure) and the upstream channel (higher pressure). Once the pressure gradient is large enough, the upstream pressure of the continuous phase surmounts the pressure inside the dispersed phase. Now, the continuous phase can enter the downstream channel/constriction again and thereby deforms ("squeezes") the interface (see Figure 2.5 (top d)). This continues until finally, droplet breakup occurs (see Figure 2.5 (top e)) and the process starts again (see Figure 2.5 (top a)). Since droplet breakup is mainly determined by the channel geometry, this regime is sometimes called the "geometry-controlled" regime. Typically, the droplets are larger than the dimension of the channel/constriction and are highly monodisperse [Bar10, Zha11, Zhu16].

As already mentioned before, the droplet breakup in the dripping and the jetting regime is governed by capillary instabilities. This means that instead of a pressure gradient, the viscous force of the continuous phase that streams alongside the droplet deforms the interface. Moreover, droplet breakup already occurs before the channel/constriction is filled completely with the dispersed phase (see Figure 2.5 (bottom left a, b, and c and bottom right a, b, and c)). However, like the pressure gradient before, the viscous force increases when the dispersed phase more and more fills the downstream channel/constriction. The droplet breakup of the dripping and jetting regime is also similar to that of the squeezing regime in that the droplets are formed when the continuous phase starts to deform the interface (see Figure 2.5 (bottom left $\mathrm{d}$ and bottom right $\mathrm{d}$ )). In the dripping regime, breakup occurs close to the nozzle of the dispersed phase (cross-flow and co-flow geometry) or close to the constriction (flow-focusing geometry) (see Figure 2.5 (bottom left e)). In the jetting regime, on the other hand, there is an extended stream of the dispersed phase into the downstream channel (see Figure 2.5 (bottom 
right e)). The droplet breakup is then caused by Rayleigh-Plateau instabilities. However, this severely limits the monodispersity so that the droplets are less monodisperse. In contrast, the droplets are monodisperse when they are formed in the dripping regime. In both the dripping and the jetting regime, the droplets are typically smaller than the dimension of the channel/constriction [Zhu 16].

Apart from the flow rates, the droplet generation regimes can be influenced by adjusting the interfacial tension. Most commonly, this is done by adding a surfactant to the continuous phase which lowers the interfacial tension of the interface once droplet generation starts. Thus, it becomes easier to deform the interface and hence, to generate droplets. By lowering the interfacial tension, the Capillary number $\mathrm{Ca}$ is increased. This means that in order to remain in the same droplet generation regime, the flow rates have to be decreased accordingly. An important aspect of surfactant addition is the adsorption-desorption kinetics towards the interface which include surfactant mass transfer time. When the time for forming one droplet is much larger than the time for the surfactant to diffuse to the newly created interface, an equilibrium surfactant concentration at the interface is obtained. In the inverted case, on the other hand, the surfactant concentration at the interface remains quite low during droplet formation as if no surfactant was present at all. For the case of similar time frames, the result is a dynamic interfacial tension that changes during the droplet formation. Here, it becomes possible to change the droplet size by small shifts in the flow rates or the surfactant concentration [Ann16, Zhu16].

Though the geometries, forces, dimensionless numbers, and droplet generation regimes have been discussed now, what is lacking are advantages and applications of droplet-based microfluidics compared to microfluidics in general. Since the droplets offer even smaller volumes than the microfluidic channel themselves, processes can be scaled down even further. For example, the droplets can act as microreactors that prevent their content from leaving the reaction site, while also having a high interfacial area-to-volume ratio to accelerate reactions that require transfer between two droplets. Moreover, the monodisperse droplets can be produced in high numbers. Various geometries and droplet generation regimes offer a tight control over a wide range of droplet sizes, which helps to monitor the stoichiometry of a reaction. Furthermore, as the droplets can adopt the size of organelles and cells, the former ones can mimic the latter ones for studying biological processes. Two further examples for applications are the investigation of reaction kinetics, commonly of enzymes, and the synthesis of molecules with a highly exothermic energy profile where the temperature has to be precisely 
controlled. Most of the applications are difficult to undertake with any other technique if not even completely impossible. Finally, the droplets can also serve as templates for the synthesis of microcapsules, microparticles, and microfibers that themselves are used in pharmaceutics, cosmetics, and food. When the continuous phase is solidified after the droplet generation and the droplets are removed afterwards, monodisperse porous materials can be produced which was done in this PhD thesis [Chr07, Bar10, Teh08, Zhu16].

\subsection{Polymers and Porous Polymers}

\subsubsection{Polymers}

According to the International Union of Pure and Applied Chemistry (IUPAC), a polymer is defined as "[a] molecule of high relative molecular mass, the structure of which essentially comprises the multiple repetition of units derived, actually or conceptually, from molecules of low molecular mass" [www1]. This 'high relative molecular mass' is typically at least above $1000 \mathrm{~g} \mathrm{~mol}^{-1}$ and often even above $10000 \mathrm{~g} \mathrm{~mol}^{-1}$ [Lec10]. Therefore, adding or removing one unit does not significantly change the properties of a polymer. In the form of wood, fur, horn, proteins, or carbohydrates, naturally occurring biopolymers have been known to and used by mankind for millenia [Tie14]. In contrast, scientific research on synthetic polymers started only about 200 years ago and their widespread industrial production began roughly 80 years ago [Tie14]. Today, the most commonly used synthetic polymers are polyethylene (PE), polystyrene (PS), polyvinylchloride (PVC), poly(methyl methacrylate) (PMMA), polytetrafluoroethylene (PTFE), and polyvinyl alcohol (PVA) [Lec10].

\section{Types of Polymers and Polymerizations}

If a polymer only consists of one singular repeating unit, it is called a homopolymer, while two or three repeating units mean that the polymer is a copolymer or terpolymer, respectively [Tie14]. Furthermore, using the example of a copolymer, four different types can be distinguished by the alternating sequence of the two monomers inside the polymer. These four types are statistical/random copolymers, alternating copolymers, block copolymers, and graft copolymers and are all schematically depicted in Figure 2.6 (left) [Tie14]. 

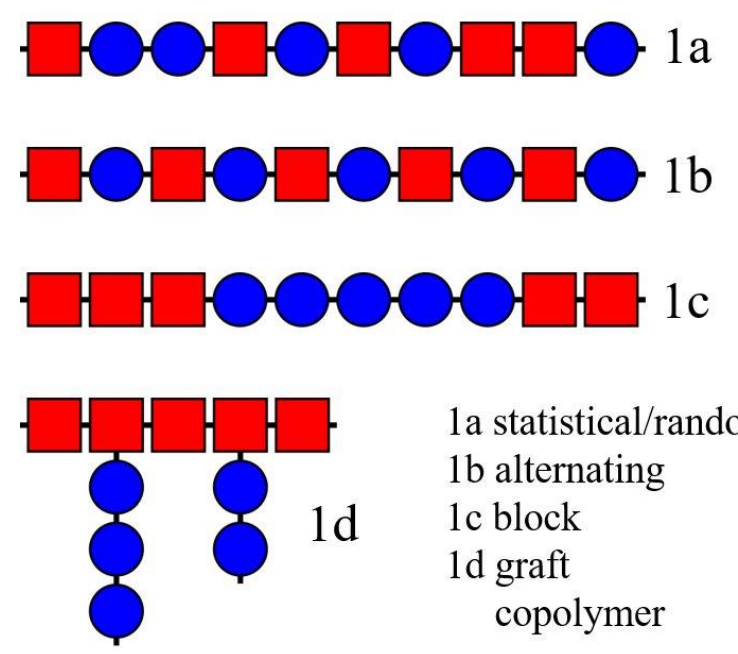

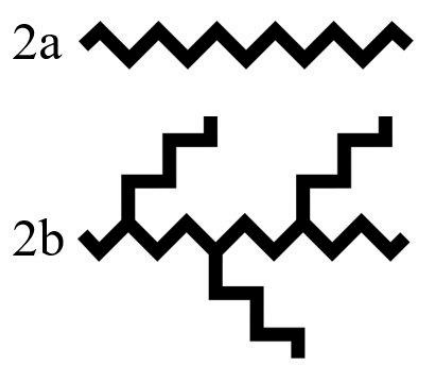

2a linear

$2 b$ branched

$2 \mathrm{c}$ crosslinked

$2 \mathrm{~d}$ star-shaped

polymer

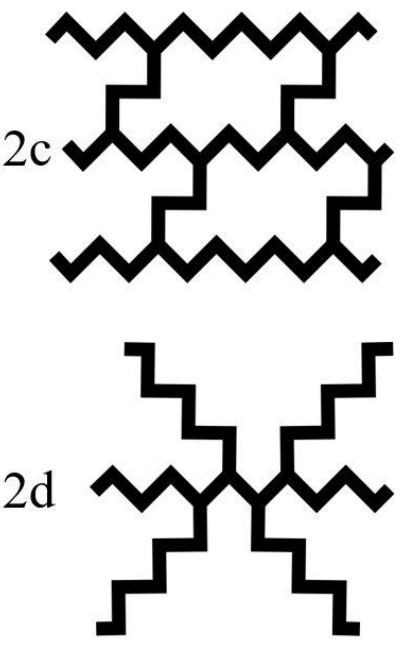

Figure 2.6: Schematical depiction of (left) statistical/random (1a), alternating (1b), block (1c), graft (1d) copolymers and of (right) linear (2a), branched (2b), crosslinked (2c), star-shaped (2d) polymers.

Moreover, polymers can be distinguished according to their constitution. Here, four types exist as well. These are linear polymers, branched polymers, crosslinked polymers, and star-shaped polymers and are all depicted in Figure 2.6 (right) [Lec10, Tie14]. Another way to characterize polymers is by the manner in which they are synthesized. In general, step-polymerization and chain-polymerization can be distinguished. In step-polymerizations, the link between two monomers is made through a reaction of their two respective functional groups. These are typically hydroxy $(-\mathrm{OH})$, carboxyl $(-\mathrm{COOH})$, amine $\left(-\mathrm{NH}_{2}\right)$, and aldehyde $(-\mathrm{CHO})$ groups [Lec10] and every monomer has at least two functional groups [Lec10, Su13]. At first, two monomers are linked to form a dimer which itself then reacts with another monomer or dimer to become a trimer or tetramer, respectively. Over time, more and more smaller units are linked to form oligomers and finally polymers [Lec10, Tie14]. Step-polymerization can be further subdivided into polycondensation and polyaddition [Lec10, Tie14]. In polycondensations, every reaction step produces a low-molecular byproduct which is often $\mathrm{H}_{2} \mathrm{O}$. Examples for polymers synthesized via polycondensation are polyesters like polyethylene terephthalate (PET) and polyamides like Nylon ${ }^{\circledR}$. In contrast, no byproduct is formed in polyadditions. Here, the most prominent example are polyurethanes [Lec10].

In chain-polymerizations, the monomers typically contain at least one double or triple bond whose electrons are used to connect two molecules by forming a new bond [Su13]. However, initiators that activate some of the monomers by forming radicals are required to start the polymerization. The newly formed radical at the monomer then can react with the next monomer which itself now becomes a radical. Only at these 'active centers' other monomers 
can be added to the growing polymer chain. The most common initiators are molecules that dissociate into radicals when they are exposed to UV-light or heat and are called radical initiators [Su13]. In turn, the polymerization is called a radical polymerization. Other initiator types include anionic initiators, cationic initiators, and coordinative initiators [Tie14]. Chainpolymerizations are comprised of three main reaction steps: initiation, propagation, and termination. For the sake of convenience, the formation of a linear homopolymer with a radical initiator will be used as an example to explain the three reaction steps. Overall, the three reaction steps can be distinguished by how the concentration of radicals changes during each step. During initiation, initiator molecules dissociate into radicals and thus, the concentration of radicals increases. These radicals then activate the double bonds of the monomers by forming a new bond with one carbon atom and creating a radical at the other carbon atom. The new radical, in turn, bonds to the next monomer molecule and thereby continues the process. Figure 2.7 depicts this process for the first few steps with styrene as monomer and azobisisobutyronitrile (AIBN) as radical initiator.
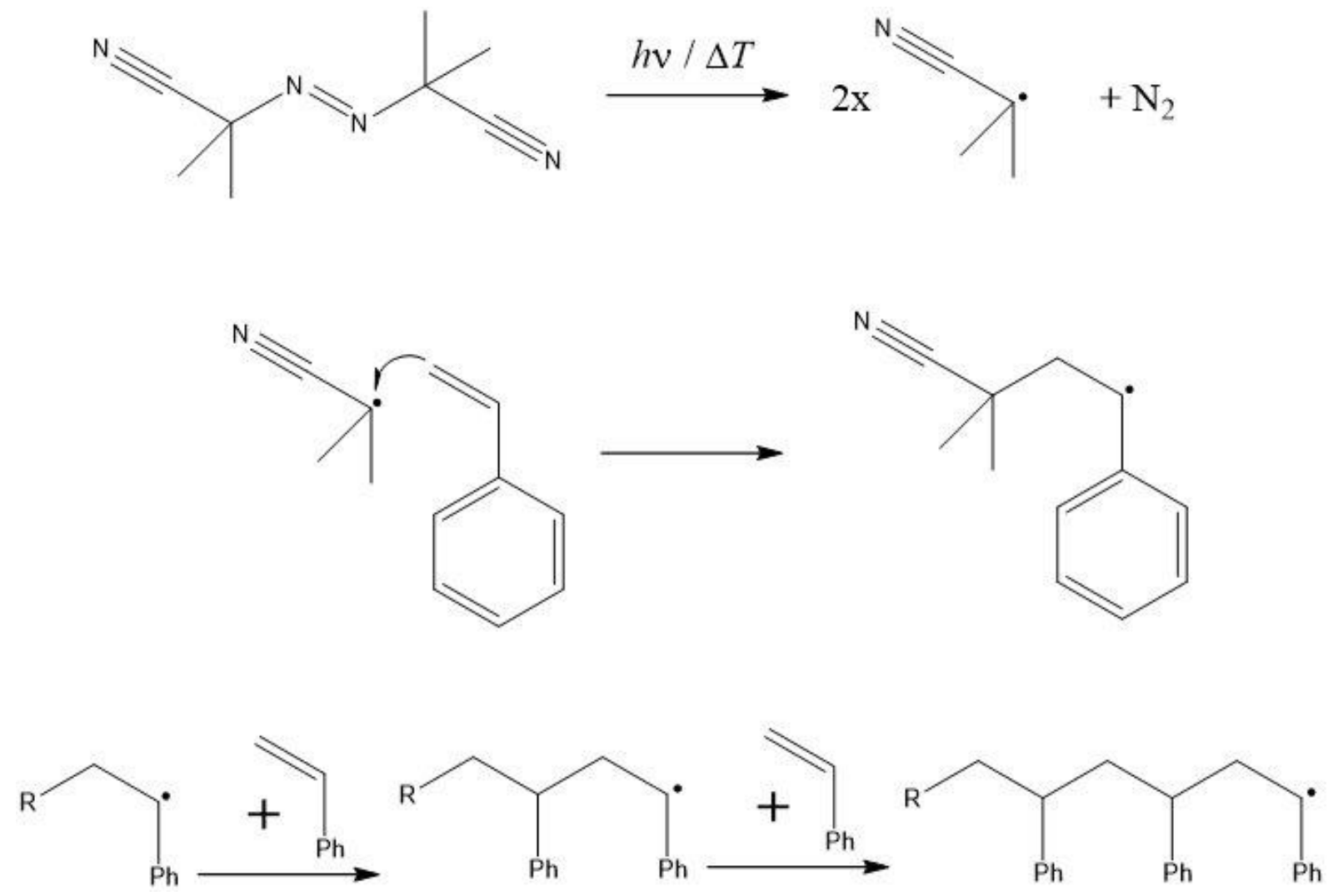

Figure 2.7: (top) Dissociation of the radical initiator AIBN by light or heat into two radicals and nitrogen, (middle) reaction of the monomer styrene with one radical, (bottom) continuing polymerization with two more styrene monomers. 
These last two steps are still part of the initiation as long as the overall concentration of radicals still increases. However, the formation of new radicals is always counteracted by radical recombination where two radicals react and thus eliminate each other. Each of the two radicals can be located at a large polymer chain, at a smaller oligomer, or still at the original dissociated initiator. After the polymerization has continued for a certain time, the formation of new radicals is compensated by radical recombination. Thus, the concentration of radicals remains constant. This is known as propagation where the overall length of the polymer chains increases. Once most of the monomer molecules are consumed, radical recombination becomes ever more likely and thus, the concentration of radicals decreases. This is further supported by the fact that with ongoing polymerization, the system will run out of initiator molecules at some point. The final step is known as termination after which the polymerization is finished [Lec10, Su13, Tie14].

\section{Polymerization Media}

Another important factor in any polymerization is the medium in which it takes place. Polymerizations without a solvent are rarely used because it is virtually impossible to remove the excess energy created by the exothermic polymerization process. This problem becomes worse when the viscosity of the solution increases due to the formation of large molecules during the polymerization. Even for polymerizations that take place in a non-polymerizable medium, the increase in viscosity during the polymerization can be problematic. The diffusion of large molecules becomes slower with increasing size. This, in turn, decreases the likelihood of radical recombination because two large and active polymer chains do not come into contact with each other anymore. However, since new radicals still form by initiator dissociation and monomers can still diffuse to the immobile, yet active polymer chains, the exothermic polymerization gets accelerated. This creates a lot of excess, uncompensated energy that manifests as a sharp increase in temperature. Overall, this phenomenon is known as the Trommsdorff/gel effect. To limit the extent of the Trommsdorff/gel effect and to maintain a comparatively low viscosity during polymerization, the monomer is usually dispersed in $\mathrm{H}_{2} \mathrm{O}$ or other media. The two most prominent methods here are emulsion polymerization and suspension polymerization. In this chapter, they will be explained with styrene as hydrophobic monomer and $\mathrm{H}_{2} \mathrm{O}$ as dispersing medium and are depicted schematically in Figure 2.8. 

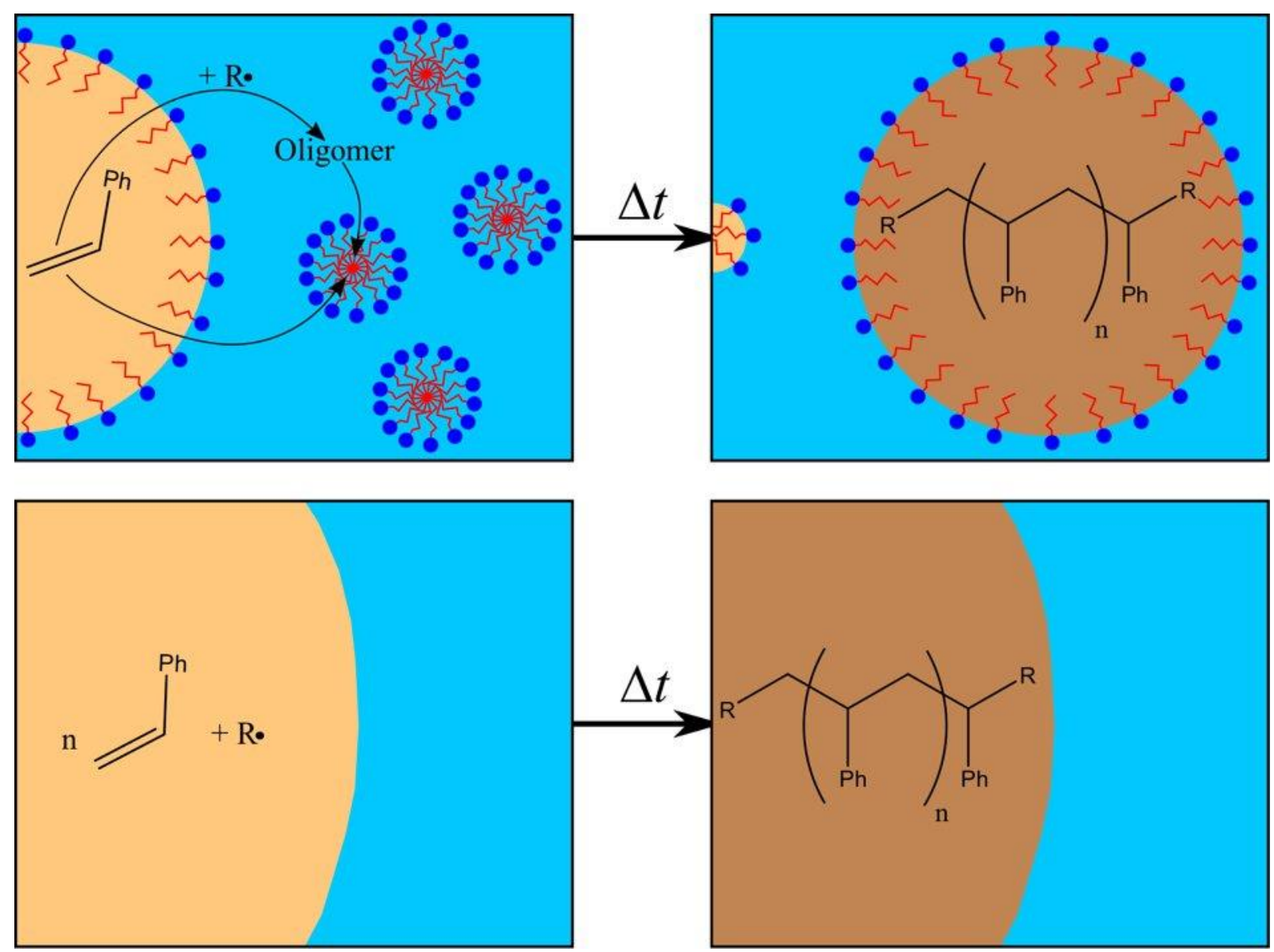

Figure 2.8: (top) Schematic depiction of emulsion polymerization: The surfactant forms micelles and stabilizes the styrene droplets. The water-soluble initiator reacts with dissolved styrene molecules to form oligomers which diffuse into the micelles. More styrene molecules diffuse into the micelles and thereby continue the polymerization. (bottom) Schematic depiction of suspension polymerization: Large, suspended styrene droplets also contain the monomersoluble initiator. Therefore, the polymerization takes place in the styrene droplets. Note that the sizes of the droplets, micelles, and surfactant molecules are not to scale.

Other, more special methods such as miniemulsion polymerization, monomolecular plain polymerization, and interphase polymerization will not be discussed here. For more information on this subject, the reader is referred to [Lec10]. In emulsion polymerization, the surfactant plays two roles, i.e. it stabilizes monomer droplets and it forms micelles in $\mathrm{H}_{2} \mathrm{O}$ (see Figure 2.8 (top left)). As already mentioned, the monomer is hydrophobic, but note that it still has a nonnegligible solubility in $\mathrm{H}_{2} \mathrm{O}$. The polymerization is started by a water-soluble initiator and mainly happens inside the micelles because the number of micelles $\left(\sim 10^{18} \mathrm{~L}^{-1}-10^{21} \mathrm{~L}^{-1}\right)$ is several orders of magnitude higher than the number of monomer droplets $\left(\sim 10^{10} \mathrm{~L}^{-1}-10^{14} \mathrm{~L}^{-1}\right)$ [Lec10, Su13]. Because of this difference in numbers, it is far more likely that an active polymer chain and a monomer meet inside a micelle than for an active polymer chain to diffuse into a monomer droplet. Note that the first reaction steps between the water-soluble initiator and a 
small number of monomer molecules occurs outside the micelles in $\mathrm{H}_{2} \mathrm{O}$ [Lec10 (page 167)]. When these short molecules grow in size, they become more hydrophobic and diffuse into close-by micelles (see Figure 2.8 (top left)). Via the surfactant concentration, the number of micelles can be adjusted which, in turn, controls the size of the resulting polymer globules. Typically, their size is between $0.05-5 \mu \mathrm{m}$ (see Figure 2.8 (top right)). In contrast, the final globules produced by suspension polymerization are much bigger with sizes between 0.01 $0.5 \mathrm{~cm}$ (see Figure 2.8 (bottom right)). Suspension polymerization does not use surfactants, though the suspended monomer droplets can be stabilized by protective colloids. Furthermore, a monomer-soluble initiator is used which means that the polymerization occurs inside the suspended monomer droplets (see Figure 2.8 (bottom left)). Subsequently, these droplets solidify over the course of the polymerization and transform into the already mentioned polymer globules. Because the polymerization takes place in the large monomer droplets, heat dissipation is more challenging than in emulsion polymerization. This can result in the emergence of the Trommsdorff/gel effect. To prevent or limit the extent of the Trommsdorff/gel effect, constant stirring is indispensable [Lec10, Su13, Tie14].

\section{Crystallinity of Polymers}

Finally, polymers can be differentiated by their mechanical behavior and their response to temperature. Both factors depend on how the polymer chains are interconnected and if the polymer is amorphous, crystalline, or a mixture of these two (= semicrystalline). One distinction is between thermoplastics, thermosets, and elastomers. Before discussing the features of these three types of polymers, the terms 'glass transition temperature' $\left(T_{\mathrm{g}}\right)$ and 'melting temperature' ( $T_{\mathrm{m}}$ ) have to be explained. While amorphous polymers show a $T_{\mathrm{g}}$, crystalline polymers exhibit a $T_{\mathrm{m}}$. Semicrystalline polymers show both a $T_{\mathrm{g}}$ and a $T_{\mathrm{m}}$. When the amorphous part of a semicrystalline polymer or an amorphous polymer reaches its $T_{\mathrm{g}}$, it transforms from a glassy into a rubber-like state. On the other hand, when the crystalline part of a semicrystalline polymer or a crystalline polymer reaches its $T_{\mathrm{m}}$, it transforms from a crystalline state into an isotropic melt. Note that since the size of the amorphous regions or crystals varies, both $T_{\mathrm{g}}$ and $T_{\mathrm{m}}$ are not exact temperatures but rather temperature ranges. The temperature ranges at which $T_{\mathrm{m}}$ and $T_{\mathrm{m}}$ appear, respectively, can be measured by differential scanning calorimetry (DSC) or differential thermal analysis (DTA). Thermoplastics consist of linear, non-crosslinked polymer chains and are usually amorphous or semicrystalline. They are frequently soluble in organic solvents and can be converted into a polymer melt at high enough temperatures. In this state, it 
is possible to re-shape a thermoplastic which will then remain in its new shape once cooled down again. In contrast, thermosets cannot be re-shaped at all. Even if they are heated up to several hundred degrees centigrade, they will rather decompose than undergo a $T_{\mathrm{g}}$ or $T_{\mathrm{m}}$. The reason for this behavior is that thermosets consist of highly crosslinked polymer networks. This feature also explains why thermosets cannot be dissolved in any solvent and show no or only very limited swelling. Both thermoplastics and thermosets respond rigidly when they are compressed or stretched, while elastomers show - as their name already implies - elastic behavior. The reason for this is that elastomers have a $T_{\mathrm{g}}$ that lies below room temperature [Gib97], which means that they are in a rubber-like state. Elastomers consist of amorphous, lightly crosslinked polymer chains and cannot be molten. Furthermore, they are not soluble in solvents, but can be swollen by them [Gib97, Lec10, Tie14].

\section{Polystyrene and Polydivinylbenzene}

In this $\mathrm{PhD}$ thesis, only styrene and divinylbenzene (DVB) were used as monomers which is why a closer look is taken at the polymerization of styrene/DVB mixtures. Since DVB has two vinyl groups, it can be used for crosslinking. Similar to other factors like temperature, initiator concentration, and type of polymerization, the styrene/DVB ratio affects the kinetics of the polymerization. Firstly, the rate of polymerization increases linearly by a factor of $\sim 2.5$ when one changes from pure styrene to pure DVB [Sto65, Zou90]. Secondly, when both styrene and DVB are present, it is more likely for DVB to join a growing polymer chain [Sch75]. Thirdly, in bulk or suspension polymerization, the addition of DVB has an influence on the onset of the Trommsdorff/gel effect. By crosslinking with DVB, one increases the molecular weight of the polymers which simultaneously increases the viscosity of the system. Therefore, the gel point appears at lower conversion rates compared to the polymerization of pure styrene [Sto65]. This effect is most perceptible below $10 \mathrm{wt}$ \% DVB, while there is essentially no change anymore above $10 \mathrm{wt}$.\% DVB and the viscosity at a certain conversion rate even slightly decreases above $20 \mathrm{wt} . \%$ [Sto65]. The reason for this behavior is the twofold role of DVB during polymerization which depends on the styrene/DVB ratio. For DVB fractions $<10 \mathrm{wt} \%$, the chains become connected chemically and thus, the mechanical stability of the whole polymer system increases [Hay83, Sun93]. Though, the polymerization is still occurring homogeneously everywhere [Sto65, Kun68, Wie82]. Above 10 wt.\% DVB, on the other hand, the crosslinker becomes a comonomer. Now, microgel particles with a net-like structure and a high degree of crosslinking are formed in the beginning of the polymerization. These structures are separated from each 
other by a pseudo-phase of monomers. Simultaneously, the monomers swell the microgel particles, thereby continuing the localized, inhomogeneous polymerization. As a rule of thumb, the extent of swelling decreases with increasing DVB fraction since it becomes increasingly difficult for molecules to diffuse into the ever more tightly crosslinked structure [Sto65, Kun68, Wil75, Hil85b]. Only when the polymerization in the microgel particles is finished, they start to become connected with each other. However, these links between the microgel particles are mechanically weaker than the interconnections inside them [Wie82] and thus, the copolymer has a lower mechanical stability than at lower DVB fractions [Sun93]. Summarized, at high DVB fractions, a sort of phase separation occurs during polymerization [Sto65, Dus67, Kun68, Wie82, Sun93]. Since the viscosity remains comparatively low during the polymerization of the microgel particles and only increases significantly once their connection starts, a precise gel point is observed here [Sto65]. In contrast, a broader gel point exists when the DVB fraction is lower since the crosslinking and therefore also the increase in viscosity happens more gradually [Sto65]. The styrene/DVB ratio furthermore determines the probability for intra- versus interchain polymerization and the fraction of pendant double bonds, i.e. double bonds that remain unreacted after the polymerization is finished. At low DVB fractions, DVB acts solely as crosslinker and mainly interchain polymerization occurs. The fraction of pendant double bonds is very low as nearly all extra vinyl groups are used for crosslinking. At high DVB fractions, on the other hand, intrachain polymerization inside the microgel particles dominates over interchain polymerization. Thus, cyclizations occur that prevent a lot of extra vinyl groups from polymerizing which, in turn, increases the fraction of pendant double bonds [Sto65, Sop72, Hil85a, Hil85b, Sun93]. Finally, the styrene/DVB ratio influences the glass transition temperature $T_{\mathrm{g}}$. At first, adding DVB to pure styrene increases the $T_{\mathrm{g}}$. For example, Zou et al. [Zou90] found that the $T_{\mathrm{g}}$ of crosslinked monodisperse polystyrene beads increases from 104,4 ${ }^{\circ} \mathrm{C}$ to $133{ }^{\circ} \mathrm{C}$ when up to $10 \mathrm{wt} . \%$ of DVB were added. At higher DVB fractions, no $T_{\mathrm{g}}$ can be observed anymore since it is impossible to transform a highly crosslinked polymer into a flowable polymer glass [Wie82].

\subsubsection{Porous Polymers}

By converting polymers into porous polymers, the spectrum of possible applications broadens immensely. Porous polymers - or polymer foams as they are often called as well - are part of the larger group of cellular solids. These colloidal materials consist of a solid continuous phase and a gaseous dispersed phase. While the gas is typically air, a wide range of solids like metals, 
ceramics, glasses, and polymers can be used to create a cellular solid. Though this chapter focusses solely on porous polymers, the reader can find information on cellular solids with other materials in Gibson et al. [Gib97]. The properties of porous polymers are given by (a) the chemistry of the polymer, (b) the volume fraction of the dispersed phase $\Phi_{\text {gas }}$, and (c) the morphology. As for the chemistry of the polymer, the chemical composition of the polymer i.e. the type of monomer(s) - is not solely decisive. Factors like the degree of crystallinity, the degree of crosslinking, and the extent of the chain length distribution of the polymer play their parts as well. The volume fraction of the dispersed gas $\Phi_{\text {gas }}$, i.e. how much gas is dissolved in the polymer matrix, is closely related to the relative density of the porous polymer $\rho_{\text {rel }}$ and the porosity of the porous polymer $P$. It holds

$$
\rho_{\text {rel }}=\frac{\rho_{\text {porous polymer }}}{\rho_{\text {polymer }}}
$$

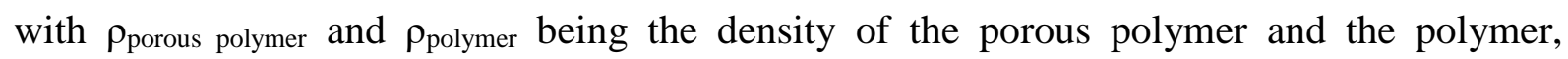
respectively. In turn, $P$ is calculated from $\rho_{\text {rel }}$ via

$$
P=1-\rho_{\text {rel }} \text {. }
$$

When neglecting the mass of the dispersed gas $m_{\text {gas }}$, $\rho_{\text {rel }}$ and $P$ can easily be expressed with

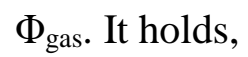

$$
\rho_{\text {rel }}=1-\Phi_{\text {gas }}
$$

and

$$
P=\Phi_{\text {gas }}
$$

A detailed derivation of how $\Phi_{\text {gas }}$ relates to $\rho_{\text {rel }}$ and $P$ is given in the Appendix in Section 6.1 (a). The 'morphology' summarizes all characteristic features that are associated with the structure. These are the average pore size and pore size distribution, the pore shape, the texture and thickness of the pore walls, and the interconnectivity of the pores. The last term describes whether the pores are connected by windows or closed off from each other and what size the windows have. Note that while this $\mathrm{PhD}$ thesis uses the terms 'pores' and 'windows', other works [Gib97, Lan95, Lee07, Mil07] use the terms 'cells' for the larger voids and 'pores' for the interconnections between these voids, respectively. Furthermore, note that the pore size subdivides porous polymers into macroporous polymers (pore width $>50 \mathrm{~nm}$ ), mesoporous 
polymers $(2 \mathrm{~nm} \leq$ pore width $\leq 50 \mathrm{~nm})$, and microporous polymers (pore width $<2 \mathrm{~nm}$ ) [Eve72, Bur76]. In this $\mathrm{PhD}$ thesis, only macroporous polymers were synthesized [Gib97, Lan95].

\section{Applications of Porous Polymers}

Since porous polymers display a broad range of properties different from their bulk counterparts, this opens up numerous different applications and explains their widespread use. The most important benefit of porous polymers is that they are much lighter than the bulk polymer. However, porous polymers can still exhibit a substantial mechanical stability which is why they are widely used as packaging materials where they protect commodities from physical damage by absorbing impact energy. Apart from commodities, it is also possible to protect body parts with porous polymers which is used in protective sports gear like shin guards for football players or helmets for cyclists, construction workers, skiers, etc. If a porous polymer is more elastic than stiff, it can be utilized for applications that involve cushioning like cushions or the sole of running shoes. Another property of porous polymers is their insulation potential that depends on the interconnectivity of the pores. Open-pore porous polymers are used for acoustic insulation. While sound waves can enter the porous polymer, they cannot leave it on the opposite side and instead get damped by the pore walls. On the other hand, closed-pore porous polymers are used for thermal insulation. Convection from one side to the other is suppressed by the porous structure by repeated absorption and reflection at the pore walls. While the polymer might have a non-neglectable heat conductivity, the volume fraction of the porous polymer $\Phi_{\text {polymer }}$ is usually low compared to $\Phi_{\text {gas. }}$ The gas itself has virtually no heat conductivity. Thermal insulation is employed in buildings to reduce energy costs and for the refrigeration of food and chemicals. Another type of insulation for which both open-pore and closed-pore porous polymers can be used is electric insulation. Similar to thermal insulation, it relies on the virtually non-existing electric conductivity of the gas inside the porous polymer. Closed-pore porous polymers can also be utilized for their buoyancy since water cannot penetrate the porous polymer and the density of the porous polymer is lower than the density of water. Water wings for small children or additional floating support for water vehicles are areas of application. Another field of application for open-pore porous polymers are filters and water-repellent membranes. In filters, a liquid or gas flows through the open-pore structure which causes a retardation or even retention of some of the components. This method is used for the treatment of tap water or in gas masks. Water-repellent membranes are employed in breathable fabrics or shoes. While liquid water cannot enter from the outside because of the 
hydrophobicity of the polymer, air can pass through the material freely [Gib97, Lan95, Lee07, Mil07].

\section{Foaming Techniques}

An important aspect of porous polymers is the manner in which the gas is introduced into the polymer, i.e. how they are foamed. Here, three basic methods can be distinguished: mechanical, physical, and chemical foaming. In general, the continuous phase has to be a liquid in order to allow gas to be inserted and dispersed. Therefore, the gas is either introduced before the polymerization, i.e. into a monomeric solution, or the polymer is molten. Once the gas bubbles have formed and stabilized, a solid porous polymer is obtained by polymerization or cooling down, respectively. A straightforward foaming technique is mechanical foaming where the continuous phase is simply stirred or frothed intensively to submerse air into it and to break up this air into bubbles. As for physical foaming, both inert gases and liquids with a low boiling point can be used. Inert gases like $\mathrm{N}_{2}$ or $\mathrm{CO}_{2}$ are solubilized in a polymer melt at high temperatures and high pressures. By releasing the pressure and cooling down, the inert gas forms bubbles which are simultaneously immobilized by the solidifying polymer. Foaming with liquids with a low boiling point works similarly. The polymer melt is mixed with the liquid blowing agent which remains liquid even at high temperatures because of high pressure. When the pressure is released, the blowing agent vaporizes and thus forms gaseous bubbles. Finally, the melt is cooled down again to solidify the polymer. Initially, chlorofluorocarbons (CFCs) were used as blowing agents. After their ban, hydrocarbons like butane and pentane were chosen as replacement. However, the drawback of these compounds is that they are flammable and explosive. In chemical foaming, the blowing agent is formed either (a) during the heating of the reaction mixture or (b) during polymerization. In the first case, additives are mixed into the monomer solution or polymer melt which decompose and release a gas at higher temperatures. In the second case, a byproduct is formed during polymerization which can act as a blowing agent. For example, if the temperature and pressure are adjusted to appropriate levels, i.e. to above $100{ }^{\circ} \mathrm{C}$ and atmospheric pressure, the byproduct of most polycondensations, $\mathrm{H}_{2} \mathrm{O}$, can act as blowing agent [Lan95, Mil07]. 


\section{Mechanical Properties of Porous Polymers}

As already mentioned, the mechanical properties of porous polymers play a crucial role for their applications. In applications such as packaging/protection, insulation, or floatation, the mechanical properties are important since the material will always have to resist some kind of stress. The mechanical behavior is primarily determined by the relative density $\rho_{\text {rel }}$ of the porous polymer, secondarily by the chemistry of the polymer, and marginally by other factors that include the temperature, the type of gas, the strain rate, and anisotropy. Anisotropy can here describe morphological anisotropy like pore size and shape, structural anisotropy like the existence of pore size gradients, or material anisotropy [Gib97]. The two most important mechanical characteristics of porous polymers are the Young's Modulus $E$ and the yield strength $\sigma_{y s}$. To determine these parameters, stress-strain-curves of porous polymers are measured, where the mechanical response of a porous polymer is recorded by a load cell ( $\hat{=}$ force censor) while being compressed via a hydraulic press. Depending on the progression of the stress-strain-curve, three general types can be distinguished: elastomeric, elastic-plastic, and

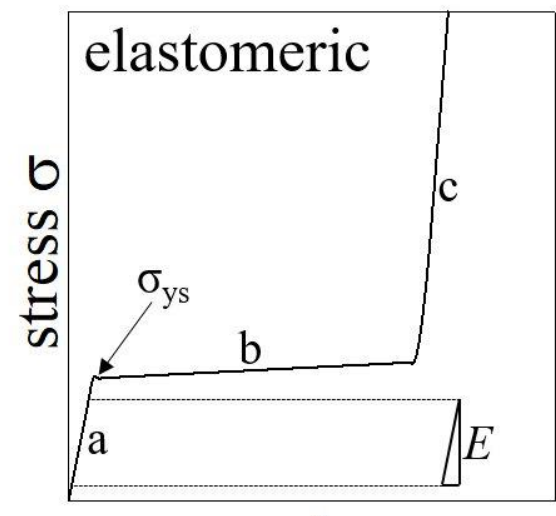

strain $\varepsilon$

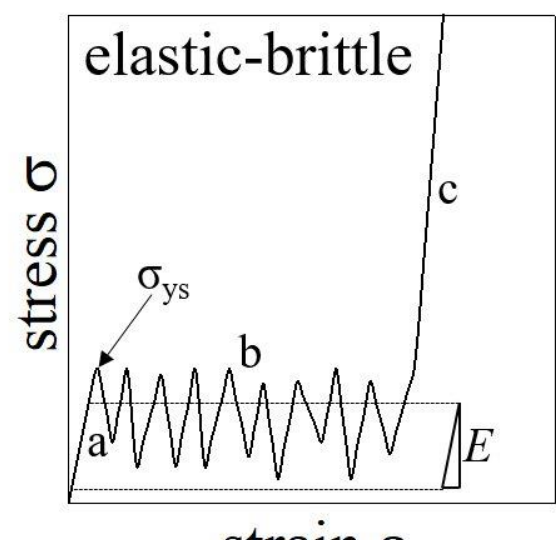

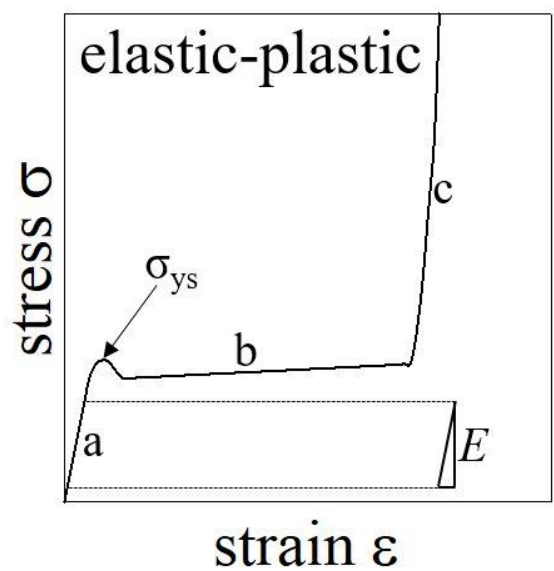

a - linear elastic region

$\mathrm{b}$ - plateau region

c-densification region

$E$ - Young's Modulus

$\sigma_{\mathrm{ys}}-$ yield strength

Figure 2.9: Schematic stress-strain-curves of (top left) an elastomeric porous polymer, (top right) an elastic-plastic porous polymer, and (bottom left) an elastic-brittle porous polymer. 
elastic-brittle. Their stress-strain-curves are drawn schematically in Figure 2.9. All three types of stress-strain-curves run through (a) a linear elastic region, (b) a plateau region, and (c) a densification region with increasing strain. The linear elastic region and the densification region look alike for all three types (see Figure 2.9 (top left a and c, top right a and c, and bottom left $\mathrm{a}$ and $\mathrm{c}$ )), while the main difference lies in the shape of the plateau region (see Figure 2.9 (top left $b$, top right $b$, and bottom left $b$ )). In the linear elastic region, the stress $\sigma$ increases proportionally with the strain $\varepsilon$. This region is dominated by the bending of pore walls (openpore) / stretching of pore faces (closed-pore) and the performed compression is usually recoverable, i.e. the porous polymer will return to its original shape once the compression is stopped. Here, the Young's Modulus $E$ is determined via the slope of the stress-strain-curve (see Figure 2.9 (top left $E$, top right $E$, and bottom left $E$ )). It holds for the stress

$$
\sigma=\frac{F}{A}
$$

with $F$ being the force acting upon the porous polymer and $A$ being the area upon which the compression is put. It holds for the strain

$$
\varepsilon=\frac{\Delta h}{h_{0}}
$$

with $\Delta h$ being the difference in height and $h_{0}$ being the initial height of the porous polymer. Thus, it holds

$$
E=\frac{\sigma}{\varepsilon}=\frac{F / A}{\Delta h / h_{0}}=\frac{F \cdot h_{0}}{A \cdot \Delta h}
$$

The higher the value of $E$ is, the more rigid is the porous polymer. Note that in order to obtain accurate results, the initial height $h_{0}$ of the porous polymers ought to be neither to large nor to small compared to the diameter or width/length of the examined sample. Furthermore, the sample ought to have dimensions at least 20 times greater than the average pore size to avoid

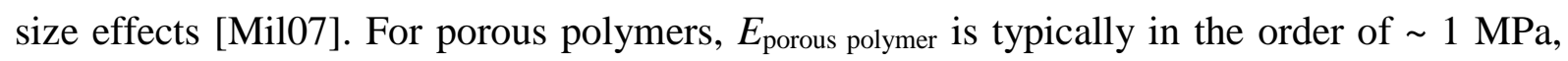

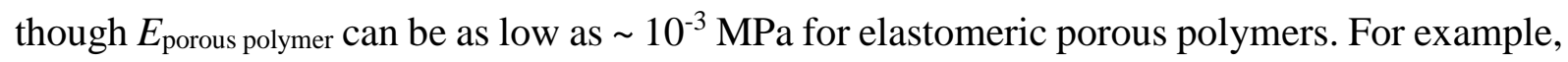
porous polystyrene with $\rho=50 \mathrm{~kg} \mathrm{~m}^{-3}$ has a $E_{\text {porous polystyrene }}=2.8 \mathrm{MPa}$ and porous polystyrene with $\rho=100 \mathrm{~kg} \mathrm{~m}^{-3}$ has a $E_{\text {porous polystyrene }}=4.8 \mathrm{MPa}$ [Rin71]. In contrast, the Young's Modulus of the bulk polymer $E_{\text {polymer }}$ usually is $\sim 10^{3} \mathrm{MPa}$. For non-porous polystyrene with $\rho=1050$ 
$\mathrm{kg} \mathrm{m}^{-3}, E_{\text {polystyrene }}=\{1400-3000\} \mathrm{MPa}$ [Gib97, page 57]. For open-pore porous polymers, the ratio between $E_{\text {porous polymer }}$ and $E_{\text {polymer }}$ is proportional to the squared relative density $\rho_{\text {rel. }}$ It holds

$$
\frac{E_{\text {porous polymer }}}{E_{\text {polymer }}}=C_{\mathrm{C}} \cdot \rho_{\text {rel }}^{2}=C_{\mathrm{C}} \cdot\left(\frac{\rho_{\text {porous polymer }}}{\rho_{\text {polymer }}}\right)^{2}
$$

with $C_{\mathrm{C}}$ being the proportionality constant which is typically $\approx 1$. For closed-cell porous polymers, an equation similar to equation (2.24) exists, although it is more complex. While equation (2.24) only takes into account pore edge bending, for closed-cell porous polymers pore face stretching and gas compression inside the pores also contribute to the mechanical behavior. It holds

$$
\frac{E_{\text {porous polymer }}}{E_{\text {polymer }}}=C_{1} \Phi^{2} \rho_{\text {rel }}{ }^{2}+C_{1}{ }^{\prime}(1-\Phi) \rho_{\text {rel }}+\frac{p_{0}\left(1-2 v^{*}\right)}{E_{\text {polymer }}\left(1-\rho_{\text {rel }}\right)}
$$

Similar to equation (2.24), $C_{1}$ and $C_{1}$ ' are proportionality constants which are both $\approx 1$. $\Phi$ is the fraction of the polymer that comprises the pore edges and thus $(1-\Phi)$ represents the fraction of the polymer residing in the pore faces. $p_{0}$ is the initial gas pressure and $v^{*}$ is the Poisson's ratio which is a measure for the "negative ratio of lateral to axial stress" [Gib97, page 187], in other words how much the pores get stretched perpendicular to and get compressed parallel to the direction of loading. For more information on the derivation of equation (2.25), the reader is referred to Gibson et al. [Gib97, pages 192-197]. At the end of the linear elastic region which is typically at a maximum strain of $\varepsilon=5 \%$, the stress-strain-curve crosses over into the plateau region. At this transition, the yield strength $\sigma_{\mathrm{ys}}$ is determined (see Figure 2.9 (top left $\sigma_{\mathrm{ys}}$, top right $\sigma_{\mathrm{ys}}$, and bottom left $\left.\sigma_{\mathrm{ys}}\right)$ ). $\sigma_{\mathrm{ys}}$ ranges between $10^{-2} \mathrm{MPa}$ and $10^{-1} \mathrm{MPa}$ for porous polymers and between $10^{1} \mathrm{MPa}$ and $10^{2} \mathrm{MPa}$ for bulk polymers. For example, porous polystyrene with $\rho$ $=50 \mathrm{~kg} \mathrm{~m}^{-3}$ has a $\sigma_{\mathrm{ys}}=0.027 \mathrm{MPa}$ and porous polystyrene with $\rho=100 \mathrm{~kg} \mathrm{~m}^{-3}$ has a $\sigma_{\mathrm{ys}}=0.062$ $\mathrm{MPa}$ [Rin71]. For non-porous polystyrene with $\rho=1050 \mathrm{~kg} \mathrm{~m}^{-3}, \sigma_{\mathrm{ys}}=\{30-35\} \mathrm{MPa}$ [Gib97, page 57]. $\sigma_{y s}$ is a measure for the maximum stress to which the porous polymer can be compressed elastically. In the plateau region, though $\varepsilon$ is successively increased, $\sigma$ remains more or less constant because pore collapse circumvents an increase of $\sigma$. For elastomeric porous polymers, pore collapse occurs via elastic buckling of the pore walls and is recoverable. In contrast, pore collapse for elastic-plastic and elastic-brittle porous polymers is irreversible. For elastic-plastic porous polymers, pore collapse occurs via plastic hinges that eventually give way to strain, while for elastic-brittle porous polymers, brittle crushing of the pore walls takes place. Eventually, the stress-strain-curve reaches the densification region once all pore walls 
have collapsed. Now, a compression of the solid polymer starts which leads to an abrupt and steep increase in $\sigma$. Other, more specialized mechanical properties like shear modulus, compressive stiffness, bending stiffness, or torsional stiffness to name but a few exist as well, but are not discussed here. Commonly, porous polymers are only used for their properties in compression, while stretching of porous polymers is rare. [Gib97, Mil07]

\section{Porous Polystyrene}

Like in Chapter 2.3.1, a closer look will be taken at porous polymers consisting of the monomers styrene and DVB. Typically, the fraction of DVB is very low $(<1 \%)$ or even zero as too much DVB would make foaming of the molten polymer impossible. Porous polystyrene (PS) is a light-weight material with closed pores, a low thermal conductivity, and a high water resistance. This is why it is used in packaging, thermal insulation, and buoyancy products [Lan95, Lee07, Mil07]. The two commercially most prominent types of porous PS are expanded polystyrene (EPS) and extruded polystyrene (XPS). EPS is manufactured via suspension polymerization of styrene that contains $\sim 5-7 \mathrm{wt} . \%$ of pentane as chemical blowing agent. Pentane decreases the $T_{\mathrm{g}}$ of PS from $\sim 100{ }^{\circ} \mathrm{C}$ to $\sim 60{ }^{\circ} \mathrm{C}$, thus enabling the expansion of the PS beads by the volatilization of pentane when the mixture is heated above the boiling temperature of pentane [Lan95, Mil07, Tie14]. XPS is produced by first melting PS, secondly adding a blowing agent, and thirdly putting the mixture under elevated temperature and pressure. Extruding the mixture leads to a decrease in pressure which volatilizes the blowing agent and a decrease in temperature which solidifies polystyrene [Lan95].

\subsection{Templating Routes}

A more specialized and indirect way of producing macroporous polystyrene in particular and of macroporous polymers in general is the use of templates. The reason for this detour is that it is easier to control and manipulate the structure of the template than to induce the desired structure/morphology by direct foaming. Obviously, the structure of the template has to be retained in the final macroporous polymer in order for this technique to be effective. At first, an emulsion or a liquid foam is formed which acts as the template for the macroporous polymer. The continuous phase is or contains the precursor for the solid polymer, i.e. the liquid monomer or the dissolved polymer. In turn, the droplets or bubbles of the dispersed phase act as placeholders for the future pores. After the formation of the template, it is solidified to fixate 
its structure. Solidification is achieved by polymerization (monomers) or gelation (polymers). Note that when emulsion templates are used, an additional drying step which removes the dispersed droplets is necessary. Figure 2.10 schematically depicts the synthesis of a porous (hydrophobic) polymer via a water-in-monomer template.

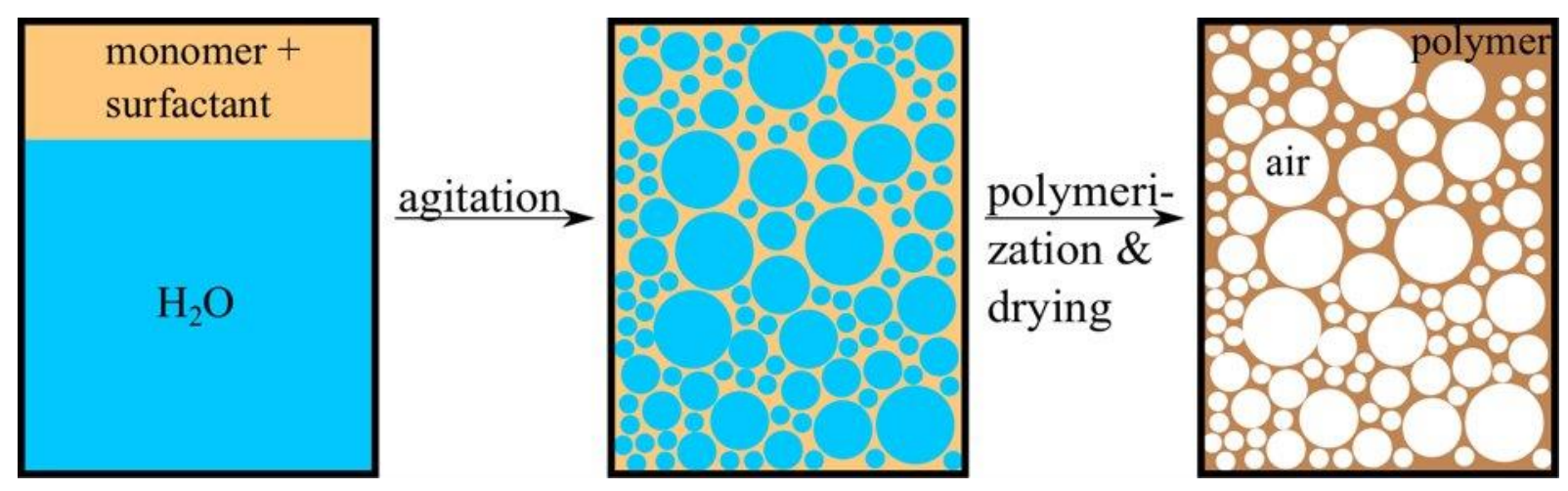

Figure 2.10: Schematic depiction of the synthesis of a porous (hydrophobic) polymer via a water-in-monomer emulsion template.

In both foam and emulsion templating, the gas/liquid or liquid/liquid interface has to be stabilized by a surfactant for at least as long as it takes to solidify the template. Therefore, no hydrophobic monomers/polymers can be used in foam templating since no surfactants that can stabilize gas-in-oil (g/o) interfaces exist. In contrast, surfactants that stabilize g/w, o/w, or w/o interfaces are well-known. In order to be able to produce highly porous materials, the volume fraction of the dispersed phase $\Phi_{\text {disp }}$ is much higher than the volume fraction of the continuous phase $\Phi_{\text {cont. }}$ As was already described in Section 2.1 (Figure 2.3), polydisperse (or monodisperse and disordered) droplets/bubbles become close-packed at $\Phi_{\text {disp }}=64 \mathrm{vol} \%$ [Dre15]. For monodisperse and ordered droplets/bubbles, the packing limit is $\Phi_{\text {disp }}=74$ vol\% . When $\Phi_{\text {disp }}>64$ vol $\%$ or $\Phi_{\text {disp }}>74$ vol\%, respectively, the droplets/bubbles lose their spherical shape and get deformed. In this $\mathrm{PhD}$ thesis, monodisperse water-in-styrene/DVB HIPEs were produced via microfluidics and consequently polymerized and dried to end up with monodisperse macroporous polystyrene/poly-DVB. Therefore, the next paragraph is about the development of (1) emulsion templating with polydisperse water-in-styrene/DVB emulsions and of (2) monodisperse foam/emulsion templating.

Though Bartl et al. published their study on what they called "umgekehrte Emulsionen" (German, in English "reversed emulsions") as early as 1962 [Bar62], it took until 1985 for Barby et al. to file for a patent on the preparation of "low density porous crosslinked polymeric materials" [Bar85]. In the following decades, the research groups of Williams [Wi188a, Wil88b, Wil89, Wil90a, Wil90b], Sherrington and Cameron [Hai91, Cam96, Cam97, Bar00, Cam00, 
Cam05, Kim11], and Silverstein [Tai01a, Tai01b, Sil05, Sil14] advanced the science of emulsion templating with styrene and DVB as the main monomers enormously. They investigated the influence of the surfactant, of the monomer/crosslinker ratio, and of $\Phi_{\text {disp }}$ on emulsion stability, morphology, and pore size. Moreover, they investigated the effect of additives like additional porogens or other co-monomers on the morphology of macroporous polystyrene and measured their mechanical behavior. Figure 2.11 shows a scanning electron microscope (SEM) picture of macroporous polystyrene synthesized with emulsion templating by Barbetta et al. [Bar00].

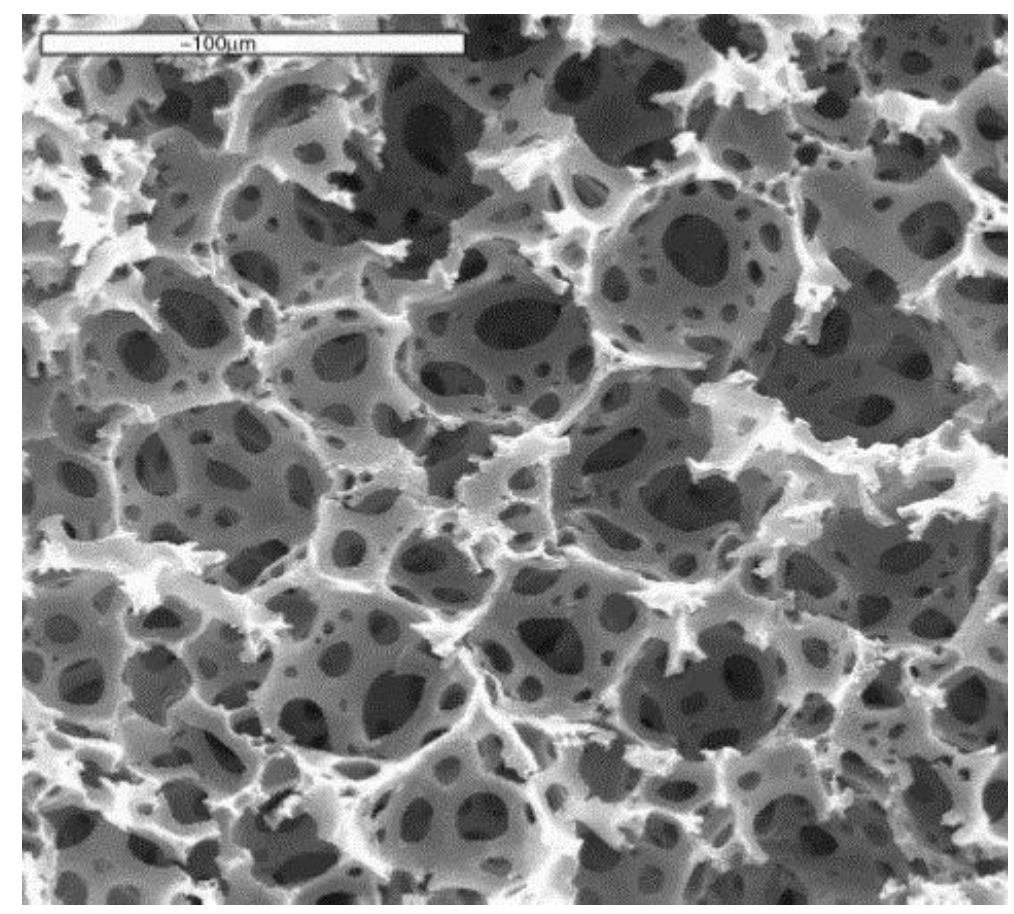

Figure 2.11: Scanning electron microscope (SEM) picture of macroporous polystyrene that was synthesized via emulsion templating by Barbetta et al. This Figure was copied from [Bar00]. Order License ID: 1096628-1.

Parallel to the development of emulsion templating, advances in microfluidics enabled researchers to produce monodisperse emulsions or foams. Talha Gokmen et al. used a monodisperse HIPE where the dispersed phase was polymerized to end up with monodisperse particles [Tal09]. In contrast, by solidifying the continuous phase and - if necessary - removing the dispersed phase, monodisperse porous materials were achieved from monodisperse foam templates [Cho09, Chu09, Lin11, Wan11, Sun12] or from a monodisperse oil-in-water emulsion template [Cos14]. Combining water-in-styrene/DVB emulsion templating with microfluidics, Quell et al. produced monodisperse macroporous polystyrene [Que15, Que16a, Que16b, Els17, Que17a, Que17b]. A schematic depiction of the synthesis route was already displayed in Figure 1.1. The most striking result was that the type of initiator - monomer- 
soluble or water-soluble - has an immense impact of the morphology. With monomer-soluble azobisisobutyronitrile (AIBN), (a) spherical pores, (b) an open-pore structure, and (c) porous pore walls with (d) smooth pore surfaces were obtained (see Figure 2.12 (bottom)). In contrast, with water-soluble potassium peroxodisulfate (KPS), (a) polyhedral pores, (b) a closed-pore structure, and (c) a three-layered pore wall with (d) small globules on the pore surfaces were obtained (see Figure 2.12 (top)). In Figure 2.12, the styrene/DVB mass ratio in the water-instyrene/DVB HIPE was 50/50, the surfactant mass fraction was $10 \mathrm{wt} . \%$, and the initiator molar fraction was $1.28 \mathrm{~mol} \%$. This composition was used in all works of Quell et al.
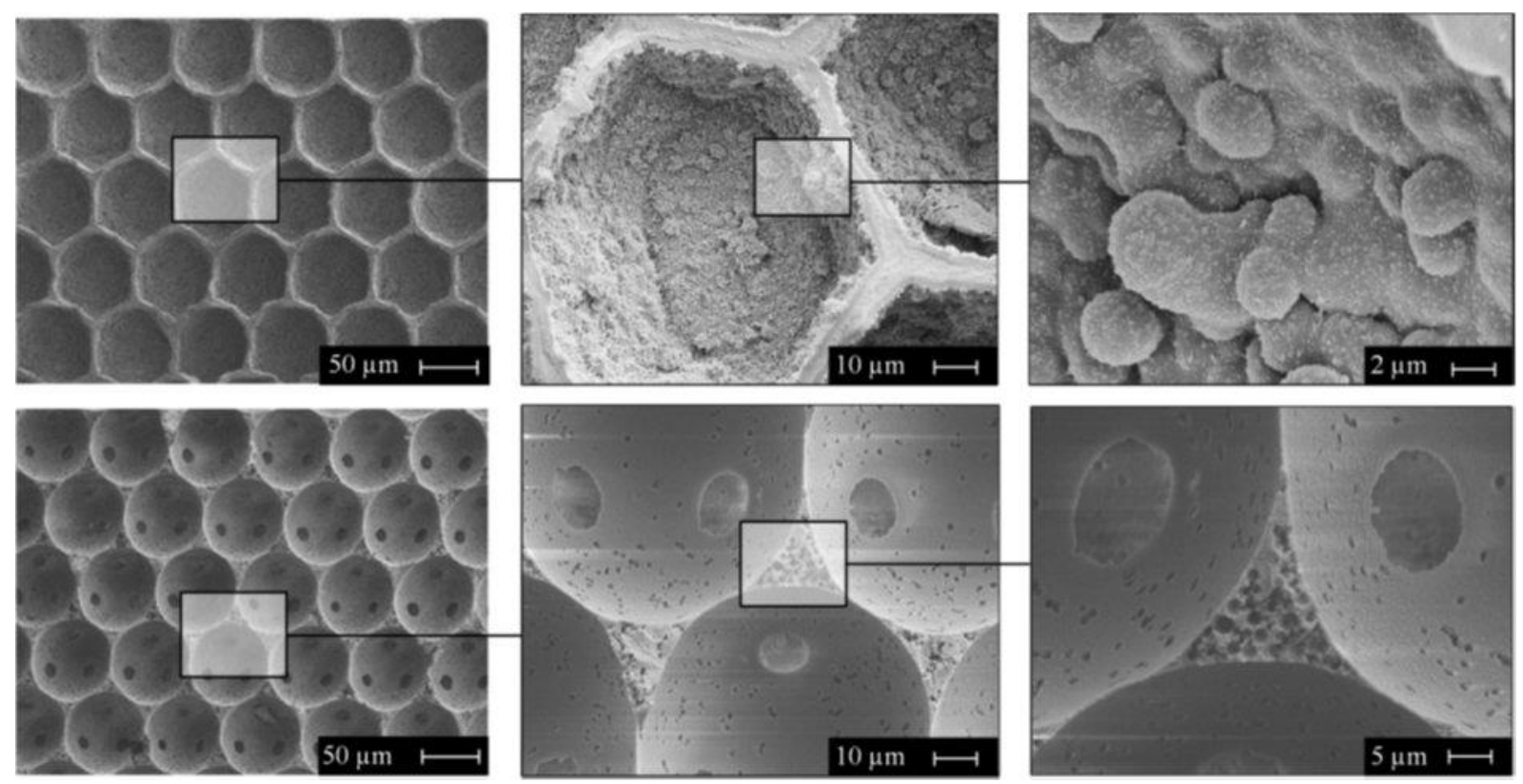

Figure 2.12: SEM pictures of macroporous PS/polyDVB obtained from monodisperse waterin-styrene/DVB HIPEs where the initiator was either (top row) water-soluble KPS or (bottom row) monomer-soluble AIBN. This Figure was copied from [Que17b]. Reprinted with permission from Quell, A.; Sottmann, T.; Stubenrauch, C.; Diving into the Finestructure of Macroporous Polymer Foams Synthesized via Emulsion Templating: A Phase Diagram Study, Langmuir 2017, 33, 537-542. Copyright (2021) American Chemical Society.

Note that the polyhedral pores appear as hexagons in two-dimensional (2D) scanning electron microscopy (SEM) pictures, while they more or less resemble rhombic dodecahedrons in three dimensions (3D). Further note that with both types of initiator, the pores remain close-packed. For monomer-soluble AIBN, the pore openings can be explained by the shrinkage of the continuous phase when styrene/DVB $\left(\rho \sim 0.94 \mathrm{~g} \mathrm{~cm}^{-3}\right)$ polymerizes to polystyrene/poly-DVB $\left(\rho \sim 1.05 \mathrm{~g} \mathrm{~cm}^{-3}\right.$ ) and consequently ruptures at its thinnest points, the films [Cam96]. Except for the pore openings, the template structure is essentially retained with monomer-soluble AIBN. With water-soluble KPS, however, two main questions arise: (1) why do the spherical droplets transform into polyhedral pores when the continuous phase is polymerized. Note that for a given 
volume, the surface area of a rhombic dodecahedron is $\sim 10.5 \%$ larger than that of a sphere (see Appendix Section 6.1 (b)). Therefore, a mechanism/force exists that increases the interfacial area and thereby counteracts the capillary pressure. The second main question is (2) why the pore walls are made up of three layers where the two outer layers appear dark and the inner layer appears lighter in SEM pictures. Quell et al. answered both questions with one explanation, namely osmotic transport of DVB from the Plateau borders into the films [Que16b, Que17a, Que17b]. According to Schwachula, it is more likely for DVB to be built into the polymer than for styrene. This means that in the beginning of the polymerization, the decrease of the DVB concentration is greater than the decrease of the styrene concentration [Sch75]. Additionally, since the polymerization starts at the water/monomer interface when watersoluble KPS is used, the decrease of the DVB concentration occurs more extensively in the films compared to the Plateau borders. Therefore, a DVB concentration gradient between the films and the Plateau borders arises because of which DVB molecules diffuse from the Plateau borders into the films. As a consequence, the size of the Plateau borders decreases, while the size of the films increases resulting in the hexagonal shape of the pore cross-sections. The threelayered pore walls were explained by Quell et al. with two outer, poly-DVB-rich layers and an inner, polystyrene-rich layer. Experiments conducted by Quell et al. [Que16b, Que17a] were in line with their hypothesis, though a quantitative way to describe the osmotic transport was missing. This is where this $\mathrm{PhD}$ thesis starts. 
-42 - 


\section{Results and Discussion}

\subsection{Disproof of Osmotic Transport}

Assuming that osmotic transport of DVB as described previously at the end of Section 2.4 is indeed responsible for the transformation of spherical droplets into polyhedral pores, one can draw the following two conclusions: (1) If the styrene/DVB mass ratio was systematically changed from 50/50 towards pure styrene, the extent of osmotic transport of DVB would decrease because the concentration gradient of DVB between the Plateau borders and the films would be lower. If the extent of osmotic transport of DVB decreases, (1a) the shape of the 2D pore cross-sections ought to change from hexagonal to more circular (polyhedral to more spherical for 3D pores). Simultaneously, (1b) the diameters of the layers inside the pore walls ought to change as well. When the styrene/DVB mass ratio is varied towards pure styrene, the diameter of the outer layers $t_{\text {outer layer }}$ (supposed to be polyDVB-rich) ought to decrease, while

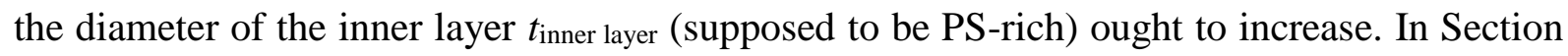
3.1.1, the styrene/DVB mass ratio was varied in steps of $10 \mathrm{wt} \%$ from $50 \mathrm{wt} . \%$ styrene $($ styrene/DVB mass ratio $=50 / 50)$ to $90 \mathrm{wt} . \%$ styrene $($ styrene/DVB mass ratio $=90 / 10)$. From now on, the styrene mass fraction $\beta_{\text {styrene }} / \mathrm{wt} . \%$ will be used instead of styrene/DVB mass ratio to avoid confusion. Note that it is not possible to polymerize a water-in-(pure) styrene HIPE as it disintegrates into two separate phases during the early stages of solidification. Further note that when $\beta_{\text {styrene }}$ is decreased towards $10 \mathrm{wt} . \%$ instead of increasing it towards $90 \mathrm{wt} . \%$, analogous results are expected. Here, the shape of the 2D pore cross-sections ought to become more circular as well since the continuous phase mainly consists of DVB, i.e. the extent of osmotic transport of DVB ought to decrease, too. Simultaneously, the thickness of the outer layer $t_{\text {outer layer }}$ ought to increase and the thickness of the inner layer $t_{\text {inner layer }}$ ought to decrease. In Section 3.1.1, only the change from $50 \mathrm{wt} . \%$ styrene to $90 \mathrm{wt} . \%$ styrene will be discussed in detail, while the change from $50 \mathrm{wt} . \%$ styrene to $10 \mathrm{wt} . \%$ styrene will be presented via selected samples in the Appendix in Section 6.2 (a).

The second conclusion that can be drawn if one believes in the existence of osmotic transport of DVB is connected to the KPS mass fraction $\beta_{\mathrm{KPS}}$. (2) When $\beta_{\mathrm{KPS}}$ is increased from its initial 2.98 wt.\%, the polymerization is sped up. Therefore, there ought to be less time for the comparatively slow diffusion process (osmotic transport of DVB). Thus, (2a) the shape of the 2D pore cross-sections should be more circular and (2b) the apparent visual difference between outer and inner layer should be less pronounced. When $\beta_{\mathrm{KPS}}$ is decreased from its initial 2.98 wt. $\%$, it is not immediately clear what should happen, i.e. if the $2 \mathrm{D}$ pore cross-sections remain 
hexagons or if they are transformed into some other shape. The corresponding experiments will be discussed in Section 3.1.2. Note that Quell et al. [Que15, Que16a, Que16b, Els17, Que17a, Que17b] used initiator molar fractions $\chi_{\text {initiator }}{ }^{2}$, while in this $\mathrm{PhD}$ thesis initiator mass fractions $\beta_{\text {initiator }}{ }^{3}$ were used. Thus, the values of Quell et al. were converted to $\beta_{\text {initiator }}$ (e.g. $\chi_{\mathrm{KPS}}=1.28$ mol $\%$ corresponds to $\beta_{\mathrm{KPS}}=2.98 \mathrm{wt} . \%^{4}$ and $\chi_{\mathrm{AIBN}}=1.28 \mathrm{~mol} \%$ corresponds to $\beta_{\mathrm{AIBN}}=1.82$ wt.\%) as described in the Appendix in Section 6.1 (c). Overall, the intention behind the variation of both $\beta_{\text {styrene }}$ and $\beta_{\text {KPS }}$ was two-fold. The first purpose was to test the hypothesis of osmotic transport of DVB. The second purpose was to collect more data about the morphology of the polymerized monodisperse water-in-styrene/DVB HIPEs and to ideally develop a more quantitative description of the osmotic transport of DVB. The results of Section 3.1 were also published in a paper in Colloid and Polymer Science [Koc20].

\subsubsection{Variation of Styrene Mass Fraction $\beta_{\text {styrene }}$}

In this Section, $\beta_{\text {styrene }}$ of the water-in-styrene/DVB HIPEs was varied from $50 \mathrm{wt} . \%$ to $90 \mathrm{wt} . \%$ in steps of $10 \mathrm{wt} . \%$. $\beta_{\mathrm{KPS}}$ was set to a constant value of $2.98 \mathrm{wt} . \%$ and $\beta_{\text {surfactant }}$ (mass surfactant divided by combined mass of monomers styrene and DVB) was set to a constant value of 10 wt.\%. For a better overview, the corresponding mass fractions are listed in Table 3.1.

Table 3.1: Type of initiator, $\beta_{\text {styrene, }} \beta_{\text {initiator, }}$ and $\beta_{\text {surfactant }}$ of the samples. $\beta_{\text {styrene was varied, }}$ while $\beta_{\text {initiator }}$ and $\beta_{\text {surfactant }}$ were kept constant.

\begin{tabular}{|c|c|c|c|}
\hline Type of Initiator & $\boldsymbol{\beta}_{\text {styrene }} / \mathbf{w t}$.\% & $\boldsymbol{\beta}_{\text {initiator }} / \mathbf{w t} \mathbf{\%}$ & $\boldsymbol{\beta}_{\text {surfactant }} / \mathbf{w t}$. \% \\
\hline water-soluble KPS & $\mathbf{5 0}$ & 2.98 & 10 \\
\hline water-soluble KPS & $\mathbf{6 0}$ & 2.98 & 10 \\
\hline water-soluble KPS & $\mathbf{7 0}$ & 2.98 & 10 \\
\hline water-soluble KPS & $\mathbf{8 0}$ & 2.98 & 10 \\
\hline water-soluble KPS & $\mathbf{9 0}$ & 2.98 & 10 \\
\hline monomer-soluble AIBN & $\mathbf{5 0}$ & 1.82 & 10 \\
\hline monomer-soluble AIBN & $\mathbf{7 0}$ & 1.82 & 10 \\
\hline monomer-soluble AIBN & $\mathbf{9 0}$ & 1.82 & 10 \\
\hline
\end{tabular}

For the sake of comparison, some samples were synthesized with the monomer-soluble AIBN as initiator. The corresponding mass fractions are also listed in Table 3.1. The way in which the

\footnotetext{
${ }^{2} \chi_{\text {initiator }}$ is calculated by dividing the molar amount of the initiator by the combined molar amount of the monomers styrene and DVB.

${ }^{3} \beta_{\text {initiator }}$ is calculated by dividing the mass of the initiator by the mass of the solvent.

${ }^{4}$ The conversion was calculated with $m$ (styrene $)+m(\mathrm{DVB})=m\left(\mathrm{H}_{2} \mathrm{O}\right)$. Quell et al. always used this mass ratio for their samples as well [Que15, Que16a, Que16b, Els17, Que17a, Que17b].
} 

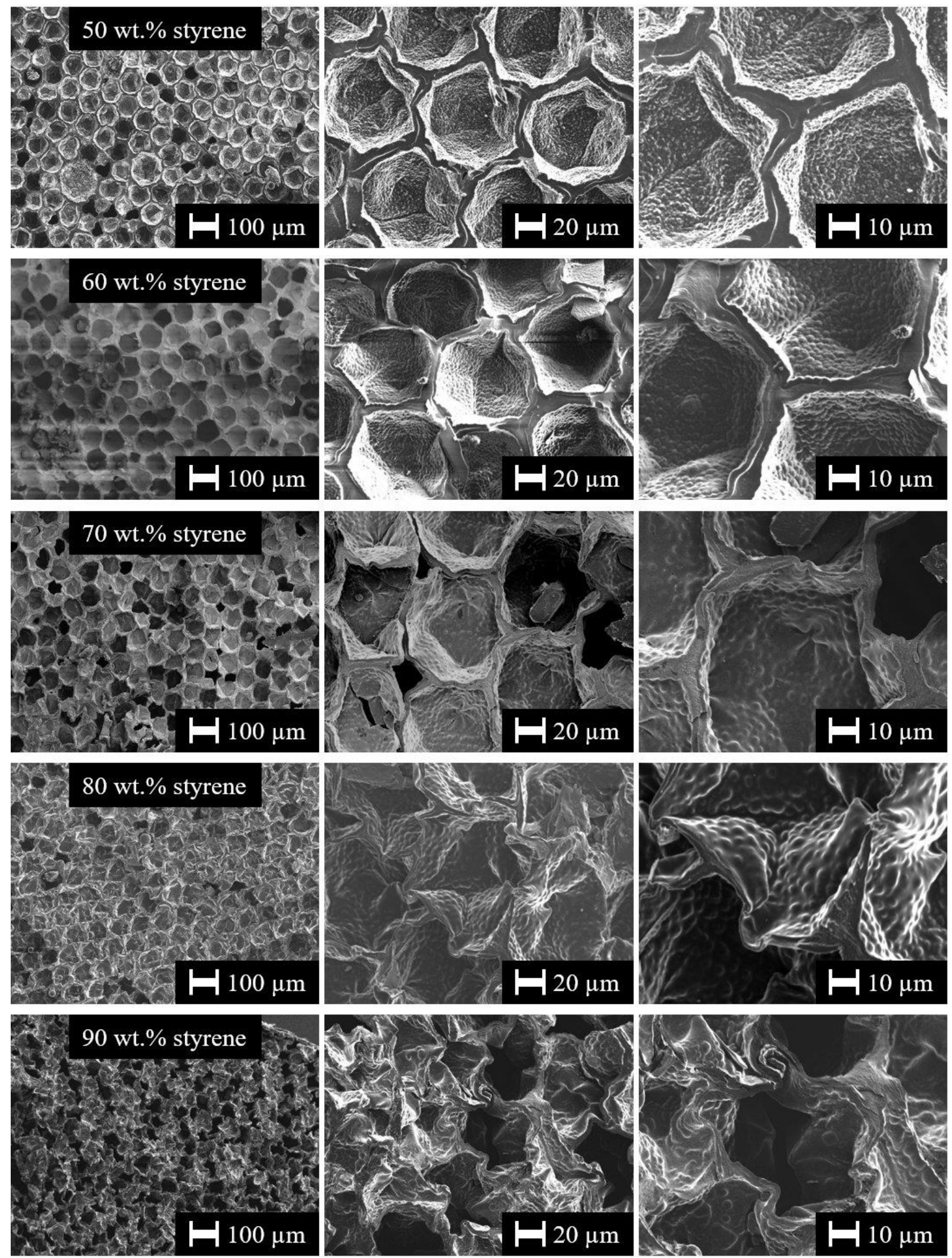

Figure 3.1: SEM pictures of macroporous PS/poly-DVB obtained via polymerization of monodisperse water-in-styrene/DVB HIPEs with $\beta_{\mathrm{KPS}}=2.98 \mathrm{wt} . \%, \beta_{\text {surfactant }}=10 \mathrm{wt} . \%$, and $\beta_{\text {styrene }}=50 \mathrm{wt} . \%,=60 \mathrm{wt} . \%,=70 \mathrm{wt} . \%,=80 \mathrm{wt} . \%$, and $=90 \mathrm{wt} . \%$. The magnifications are 100-fold (left column), 500-fold (middle column), and 1000-fold (right column). 
samples were synthesized, polymerized, and processed is described in the Experimental Section. In Figure 3.1, the SEM pictures of the KPS samples from Table 3.1 are presented with a 100-fold (left column), a 500-fold (middle column), and a 1000-fold magnification (right column). Looking at Figure 3.1, one instantly sees - contrary to the expectations - that the shape of the $2 \mathrm{D}$ pore cross-sections is not becoming more circular when $\beta_{\text {styrene }}$ is increased from $50 \mathrm{wt} . \%$ to $90 \mathrm{wt} . \%$. Instead, the shape of the 2D pore cross-sections appears to be even more "angular", i.e. it deviates even more from a circular shape. The left column of Figure 3.1 indicates that this observation is not due to a failure of the templating method since the pores are still close-packed and fairly monodisperse.

For the templating method to be fully successful, the size of the pores has to be same as the size of the preceding droplets. For both the water droplets and the pores, the diameter $d$ was calculated via the area $A$ of the $2 \mathrm{D}$ cross-sections. For circles, it holds

$$
d=2 \cdot r=2 \cdot \sqrt{\frac{A}{\pi}}
$$

with $r$ being the radius. Equation (3.1) was used to calculate $d_{\text {droplet }}$ and $d_{\text {pore. This means that }}$ although the shape of the pore cross-sections is not circular, $d_{\text {pore }}$ was calculated as if the pore cross-sections were circular. Thus, the calculation of $d_{\text {pore }}$ contains a systematic error. However, the "correct" diameter of the hexagonal 2D pore cross-sections is not clear. The distance edgeto-edge, the distance corner-to-corner, or an average of the first two can all be viewed as viable definitions for the diameter of a hexagon. In any case, the relative differences between these three values and the calculation via equation (3.1) are comparatively small as can be seen in the exemplary calculations in Appendix Section 6.1 (d). If the shape of the 2D pore cross-sections is modified from a hexagon, the determination of $d_{\text {pore }}$ via the edges and/or the corners becomes impossible. Therefore, the calculation of $d_{\text {pore }}$ that was used in this $\mathrm{PhD}$ thesis is a reasonable compromise because the occurring error is not significant. In Figure 3.2, the average diameter of the water droplets $d_{\text {droplet }}$ (black circles) in the water-in-styrene/DVB HIPEs before polymerization and the average diameter of the pores $d_{\text {pore }}$ (white squares) in the macroporous PS/polyDVB samples after polymerization are plotted as function of $\beta_{\text {styrene. }}$ 


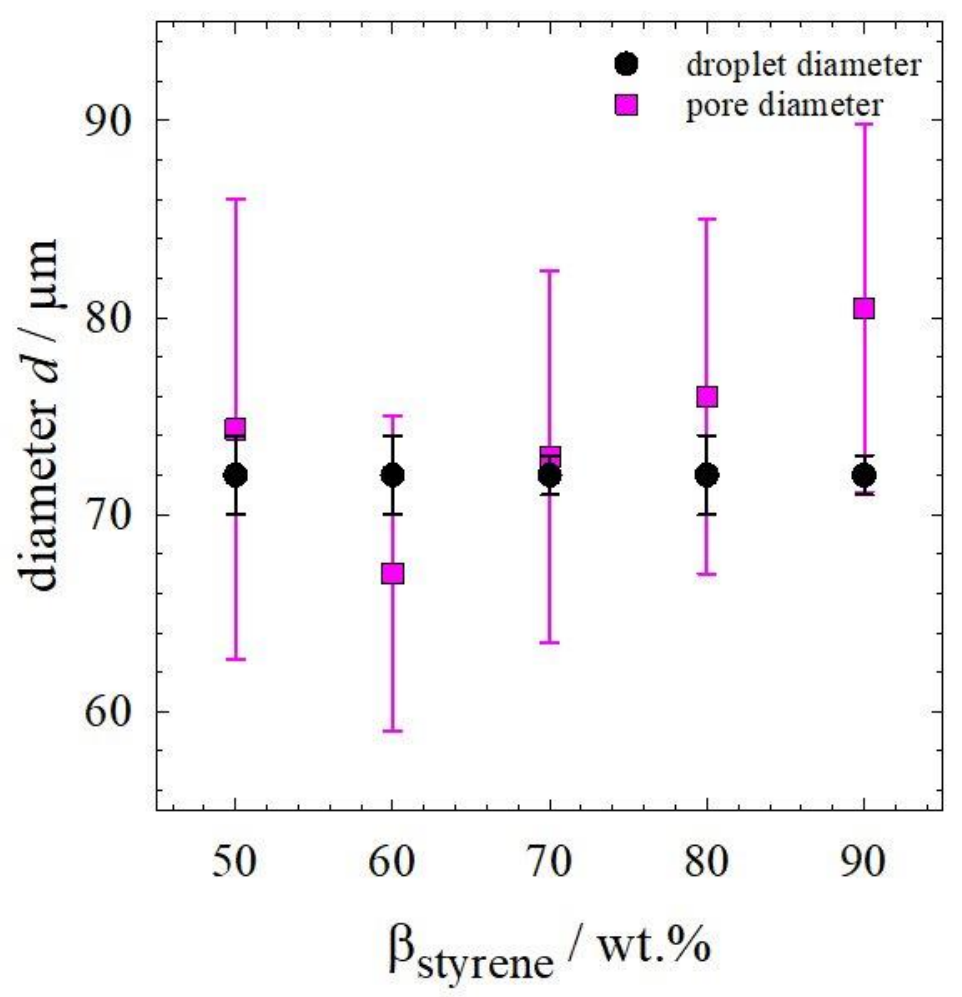

Figure 3.2: Average emulsion droplet diameter $d_{\text {droplet }}$ (black circles) and average pore diameter $d_{\text {pore }}$ (pink squares) as function of $\beta_{\text {styrene }}$ for macroporous PS/poly-DVB at $\beta_{\mathrm{KPS}}=2.98 \mathrm{wt} . \%$ and $\beta_{\text {surfactant }}=10 \mathrm{wt} . \%$.

Figure 3.2 shows that the polymerization slightly changes the average diameter and decreases the monodispersity. Before polymerization, $d_{\text {droplet }}$ is always $\sim 72 \mu \mathrm{m}$ and the water droplets are very monodisperse since the standard deviation (black error bars in Figure 3.2) is only $1-2$ $\mu \mathrm{m}$. In contrast, after polymerization the standard deviation of $d_{\text {pore }}$ has increased to values between $8-12 \mu \mathrm{m}$. Though this means that the polydispersity increases during polymerization, the pores are still far from being actually polydisperse. Concerning the change in average

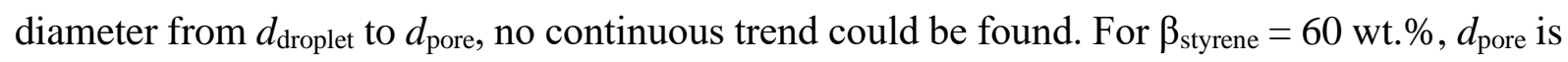

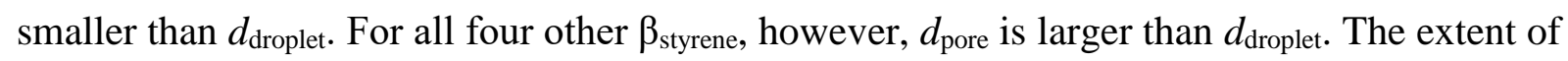
the increase is very small for $\beta_{\text {styrene }}=70 \mathrm{wt} . \%$, a little larger for $\beta_{\text {styrene }}=50 \mathrm{wt} . \%$ and $\beta_{\text {styrene }}=$ $80 \mathrm{wt} . \%$, and largest for $\beta_{\text {styrene }}=90 \mathrm{wt}$. $\%$. For all five samples, $d_{\text {pore }}$ is nevertheless always still in the range of $d_{\text {droplet. }}$ Figure 6.1 in the Appendix Section 6.2 (a) shows the development of the morphology of macroporous PS/polyDVB where $\beta_{\text {styrene }}$ was lower than $50 \mathrm{wt} \%$. At first glance, the 2D pore cross-sections remain hexagons independent of $\beta_{\text {styrene. Overall, the results }}$ from Figure 3.1 and Figure 6.1 are contrary to what should have happened if osmotic transport of DVB was the reason for the transformation from spherical droplets to polyhedral pores. 
When instead of KPS the monomer-soluble initiator AIBN is used, the 2D pore cross-sections are always circular. This can be seen in Figure 3.3 where the SEM pictures of macroporous PS/polyDVB samples that were polymerized from monodisperse water-in-styrene/DVB HIPEs with AIBN as initiator are displayed. $\beta_{\text {AIBN }}$ was set to a constant value of $1.82 \mathrm{wt} . \%, \beta_{\text {surfactant }}$ was set to a constant value of $10 \mathrm{wt} . \%$, and $\beta_{\text {styrene was }} 50 \mathrm{wt} . \%, 70 \mathrm{wt} . \%$, and $90 \mathrm{wt} . \%$. The magnifications in Figure 3.3 are 100-fold (left column), 500-fold (middle column), and 1000fold (right column).
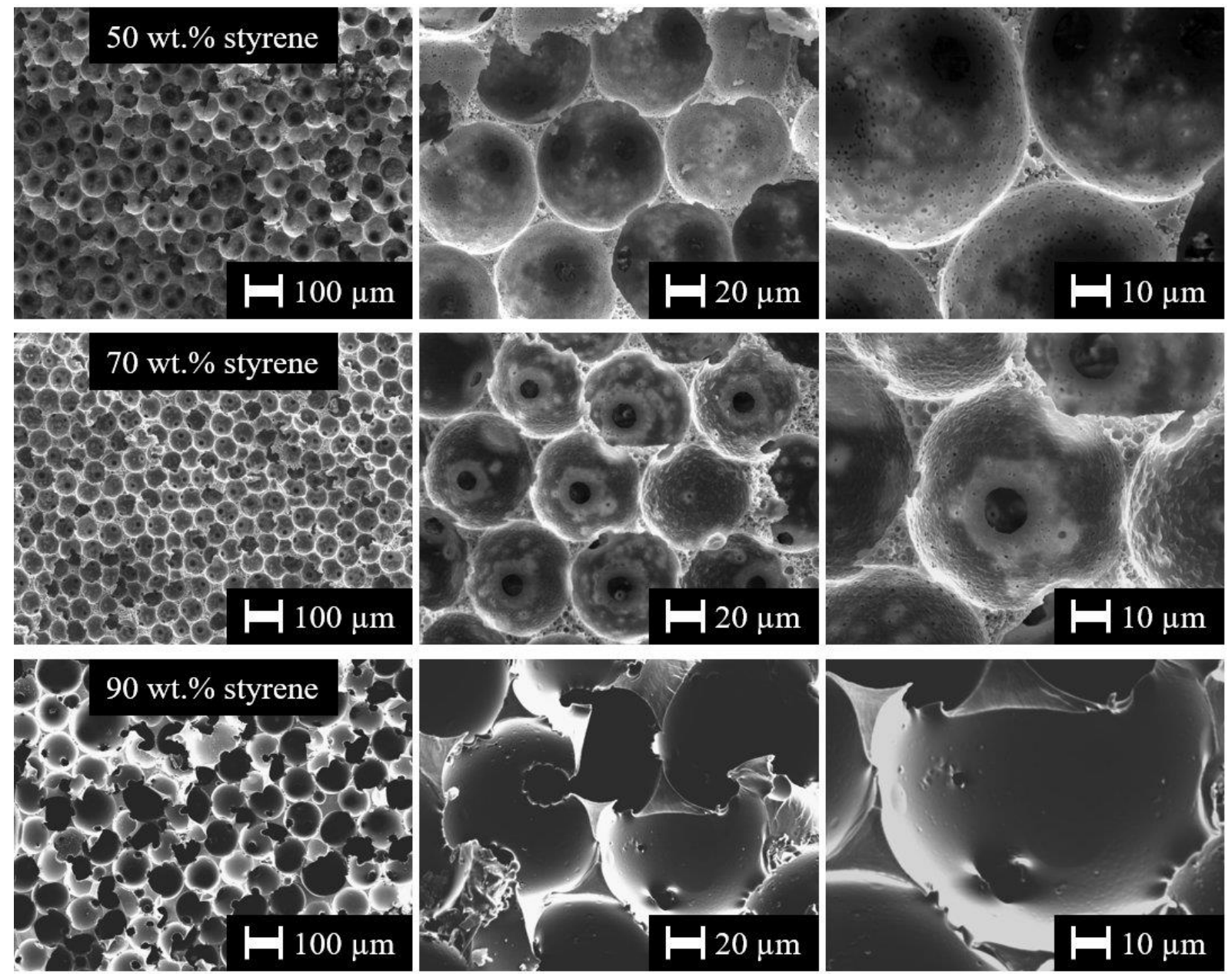

Figure 3.3: SEM pictures of macroporous PS/poly-DVB obtained via polymerization of monodisperse water-in-styrene/DVB HIPEs with $\beta_{\text {AIBN }}=1.82 \mathrm{wt} . \%, \beta_{\text {surfactant }}=10 \mathrm{wt} . \%$, and $\beta_{\text {styrene }}=50 \mathrm{wt} . \%,=70 \mathrm{wt} . \%$, and $=90 \mathrm{wt} . \%$. The magnifications are 100 -fold (left column), 500-fold (middle column), and 1000-fold (right column).

In all cases, the pores are close-packed, fairly monodisperse, and interconnected in Figure 3.3. Figure 3.4 depicts $d_{\text {droplet }}$ (black circles) before polymerization and $d_{\text {pore }}$ (pink squares) after polymerization of these samples as function of $\beta_{\text {styrene. }}$ 


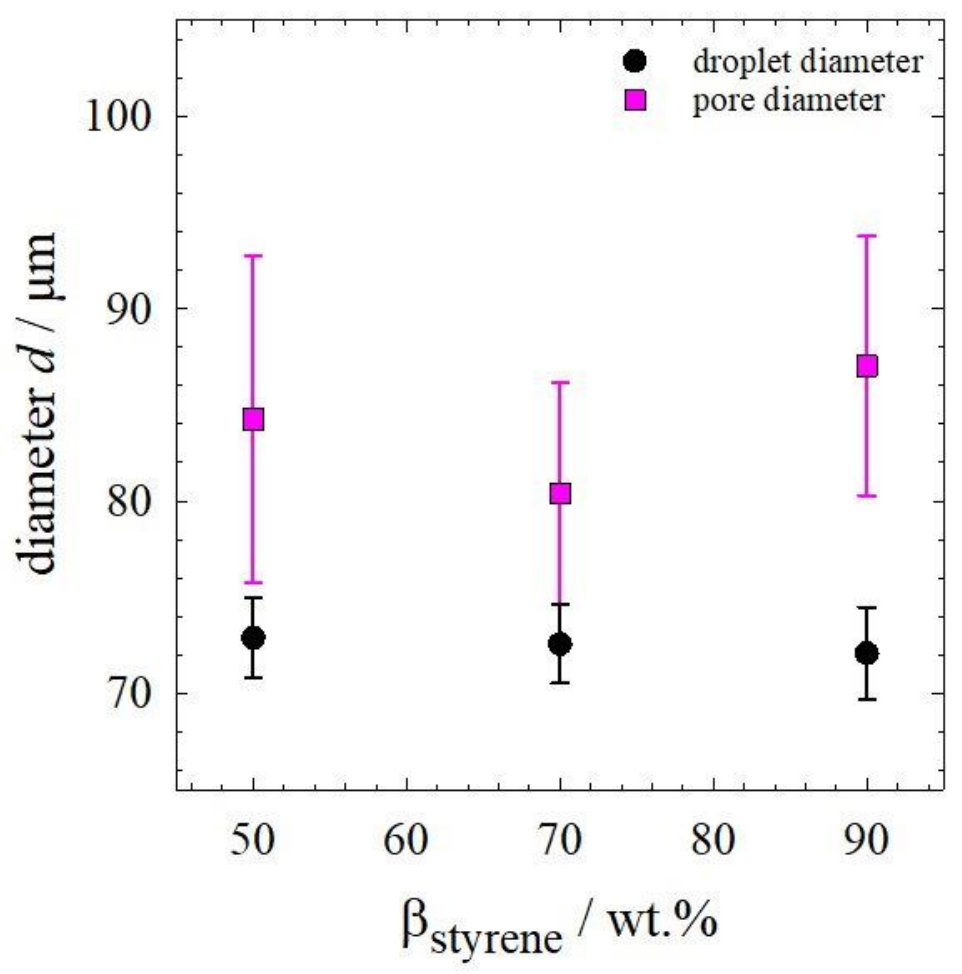

Figure 3.4: Average emulsion droplet diameter $d_{\text {droplet }}$ (black circles) and average pore diameter $d_{\text {pore }}$ (pink squares) as function of $\beta_{\text {styrene }}$ for macroporous PS/poly-DVB at $\beta_{\text {styrene }}=1.82 \mathrm{wt}$. $\%$ and $\beta_{\text {styrene }}=10 \mathrm{wt} . \%$.

As was already the case in Figure 3.2, $d_{\text {droplet }} \sim 72 \mu \mathrm{m}$ for all samples and the droplets are very monodisperse with standard deviations of only $2 \mu \mathrm{m}$. After polymerization, the standard deviation increases to values between $6-8 \mu \mathrm{m}$, meaning that the pores are still fairly monodisperse, and $d_{\text {pore }}$ has also increased compared to $d_{\text {droplet }}$. The reason for this increase might be that the water droplets are more susceptible to Ostwald ripening compared to water droplets that contain a water-soluble salt, namely KPS (see Section 2.1). Overall, it is evident that changing $\beta_{\text {styrene }}$ has little to no effect on the morphology when AIBN is used as initiator, while the morphology changes considerably as function of $\beta_{\text {styrene }}$ when KPS is used as initiator (see Figure 3.1).

\section{Shape of Pore Cross-Sections: Shape Factor $S$}

Although it is obvious from Figure 3.1 that the 2D pore cross-sections are not becoming more circular when $\beta_{\text {styrene }}$ is increased, it would be helpful to have a quantitative value to express how "circular" or "angular" the 2D pore cross-sections are. To achieve this, the ratio between the circumference $C$ and the area $A$ of a pore cross-section are taken into account. Since a circle 
has the lowest circumference-to-area ratio, this value is lowest for a circle and then increases when the shape gets more angular. The idea for such a quantitative value stems from foam science where something analogous is done in 3D with the surface area and volume of foam bubbles [Kra04, Hil04, Kra05, Kra06, Dre15]. Though only the 2D circumference $C$ and area $A$ of the pore cross-sections are used in this $\mathrm{PhD}$ thesis, the result can be taken as a representative for the 3D pores. An exemplary picture on how $C$ and $A$ were determined in one sample via the image processing software ImageJ is presented in Appendix Section 6.2 (b), Figure 6.2 (left)). However, the ratio between $C$ and $A$ is not taken directly, but two alterations are undertaken. Firstly, since $C$ scales linearly and $A$ quadratically with size, the ratio between them depends on the size of the pore cross-sections. To avoid this, the square root of $A$ is used instead of $A$. For a geometric circle, it holds

$$
\frac{C_{\text {circle }}}{\sqrt{A_{\text {circle }}}}=\frac{2 \pi r}{\sqrt{\pi r^{2}}}=\sqrt{\frac{4 \pi^{2} r^{2}}{\pi r^{2}}}=\sqrt{4 \pi}=2 \cdot \sqrt{\pi} \approx 3.545 .
$$

Next, the result of equation (3.2) is inverted to serve as a normalizing factor. This constitutes the second alteration and yields the final form to describe the shape of the $2 \mathrm{D}$ pore crosssections. In this $\mathrm{PhD}$ thesis, this value is denominated "shape factor $S$ ". It holds

$$
S=\frac{1}{2 \cdot \sqrt{\pi}} \cdot \frac{C}{\sqrt{A}}
$$

For a circle, $S=1$ (see Figure 3.5 (bottom left)). For a geometric hexagon, $S$ can be calculated from its circumference $C_{\text {hexagon }}$ and its area $A_{\text {hexagon. It holds }}$

$$
C_{\text {hexagon }}=6 \cdot a
$$

and

$$
A_{\text {hexagon }}=\frac{3 \cdot \sqrt{3}}{2} \cdot a^{2}
$$

with $a$ being the length of one edge of the hexagon. Thus,

$$
S_{\text {hexagon }}=\frac{1}{2 \cdot \sqrt{\pi}} \cdot \frac{6 a}{\sqrt{\frac{3 \sqrt{3}}{2} \cdot a^{2}}}=\sqrt{\frac{36 a^{2} \cdot 2}{4 \pi \cdot 3 \sqrt{3} \cdot a^{2}}}=\sqrt{\frac{6}{\sqrt{3} \cdot \pi}} \approx 1.050 .
$$


Consequently, $S$ ought to be $\sim 1.05$ (see Figure 3.5 (middle left)) for the hexagonal shapes found in the 2D pore cross-sections in Figure 3.1. For shapes that are distorted from a hexagon, $S \geq$ 1.05 (see Figure 3.5 (top left)). Figure 3.5 (right) depicts $S$ as function of $\beta_{\text {styrene. }}$

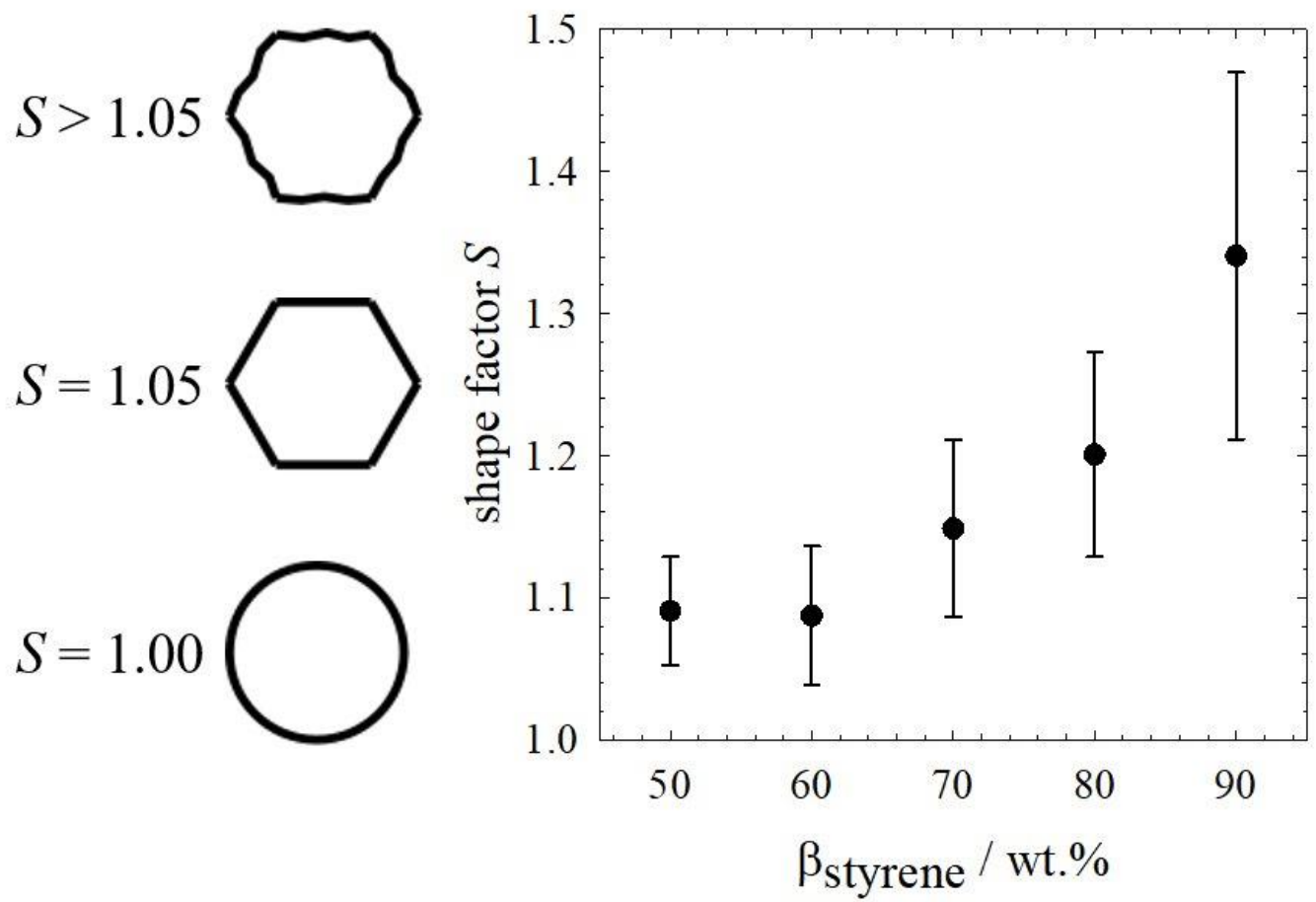

Figure 3.5: (left) Exemplary values of the shape factor $S$ for a sphere (bottom), a hexagon (middle), and a distorted hexagon (top). (right) $S$ as function of $\beta_{\text {styrene }}$ for macroporous PS/polyDVB at $\beta_{\mathrm{KPS}}=2.98 \mathrm{wt} . \%$ and $\beta_{\text {surfactant }}=10 \mathrm{wt} . \%$.

When $\beta_{\text {styrene }}$ is increased, $S$ increases from $\sim 1.09 \pm 0.04$ for $\beta_{\text {styrene }}=50 \mathrm{wt} . \%$ to $\sim 1.34 \pm 0.13$ for $\beta_{\text {styrene }}=90 \mathrm{wt} . \%$. Thus, Figure 3.5 (right) quantifies the observations from Figure 3.1, i.e.

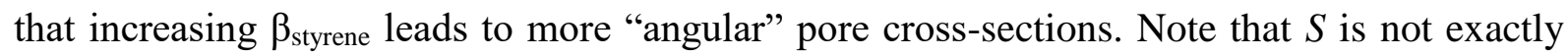
1.05 for the apparent hexagons at $\beta_{\text {styrene }}=50 \mathrm{wt} . \%$ or $\beta_{\text {styrene }}=60 \mathrm{wt} . \%$. Instead, $S \sim 1.10$. This can be explained by looking at the middle and right column of Figure 3.1. The 2D pore crosssections are not geometric hexagons with perfectly straight edges, but the edges are somewhat distorted. Thus, $C$ is larger than that of a hexagon, while $A$ stays more or less constant. As a consequence, the ratio between $C$ and $A$ is also larger and thus $\mathrm{S}>1.05$.

\section{Thickness of Pore Wall and Layers}

As already mentioned, the development of the shape of the 2D pore cross-sections is incompatible with the results that should have been obtained when osmotic transport of DVB was responsible for the transformation from spherical droplets to polyhedral pores. Another 
logical consequence of this hypothesis was that the thickness of the supposedly polyDVB-rich outer layers $t_{\text {outer layer }}$ ought to decrease and the thickness of the supposedly PS-rich inner layer

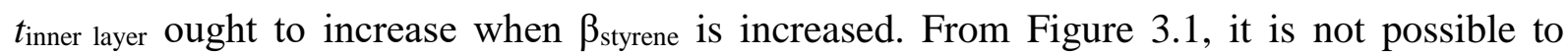

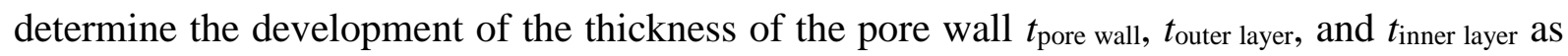

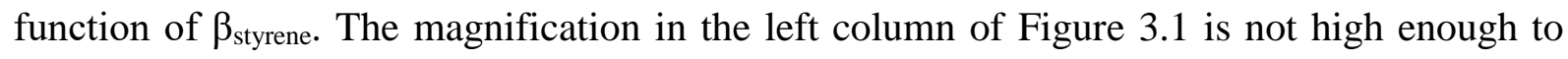
directly measure the thicknesses and the middle and right column of Figure 3.1 only show a small section of the sample. Therefore, $t_{\text {pore wall, }} t_{\text {outer layer, }}$ and $t_{\text {inner layer were determined using a }}$ SEM picture of the whole sample and consequently zooming in at various locations. An example of this method is presented in Appendix Section 6.2 (b), Figure 6.2 (right). By determining the desired length perpendicular to the direction the pore wall/layer is running

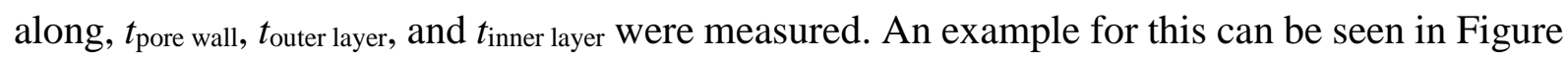
3.6.

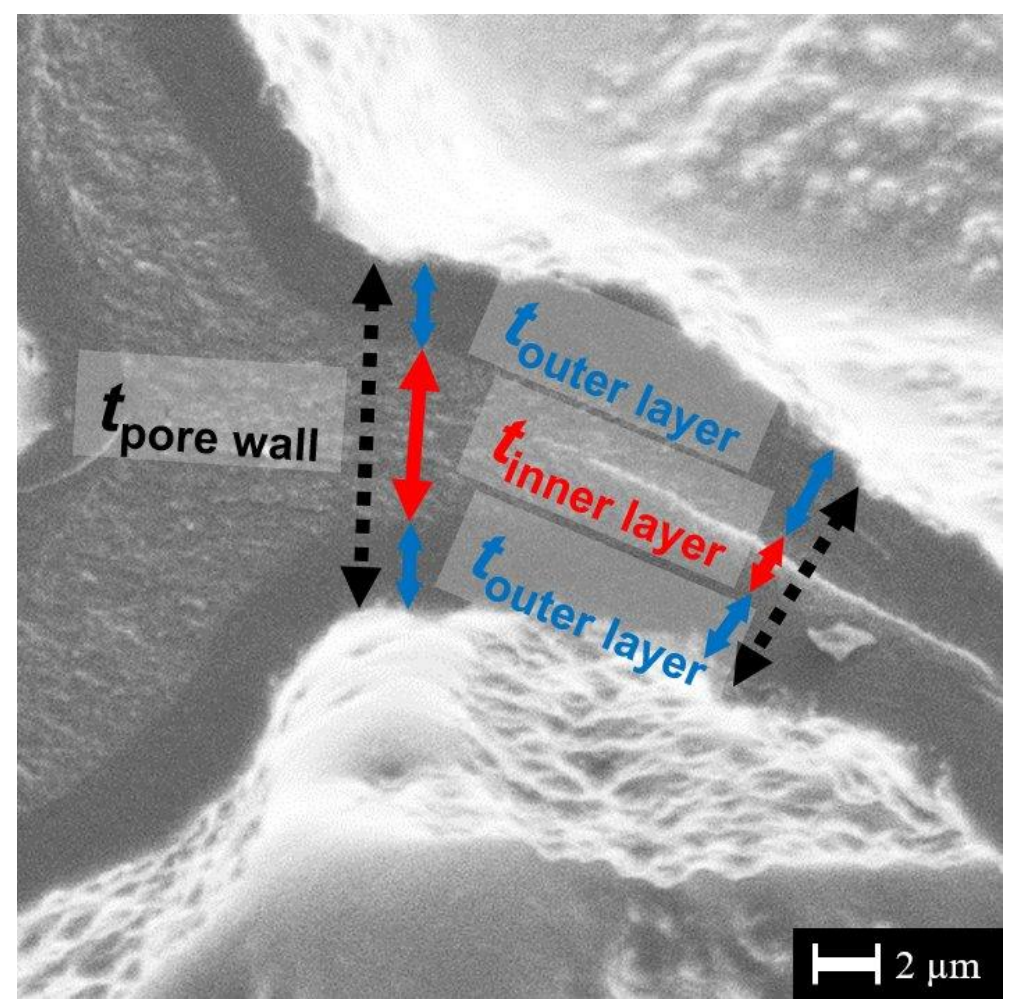

Figure 3.6: Picture of how the thickness of the whole pore wall (black), of the two outer layers (blue), and of the inner layer (red) were determined.

Figure 3.7 (top) depicts the development of $t_{\text {pore wall }}$ (black circles), $t_{\text {outer layer (blue squares), and }}$

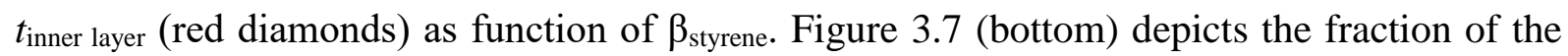
outer layer (blue squares) which is the ratio between $t_{\text {outer layer }}$ and $t_{\text {pore wall }}$ and the fraction of the inner layer (red diamonds) which is the ratio between $t_{\text {inner layer }}$ and $t_{\text {pore wall }}$. 

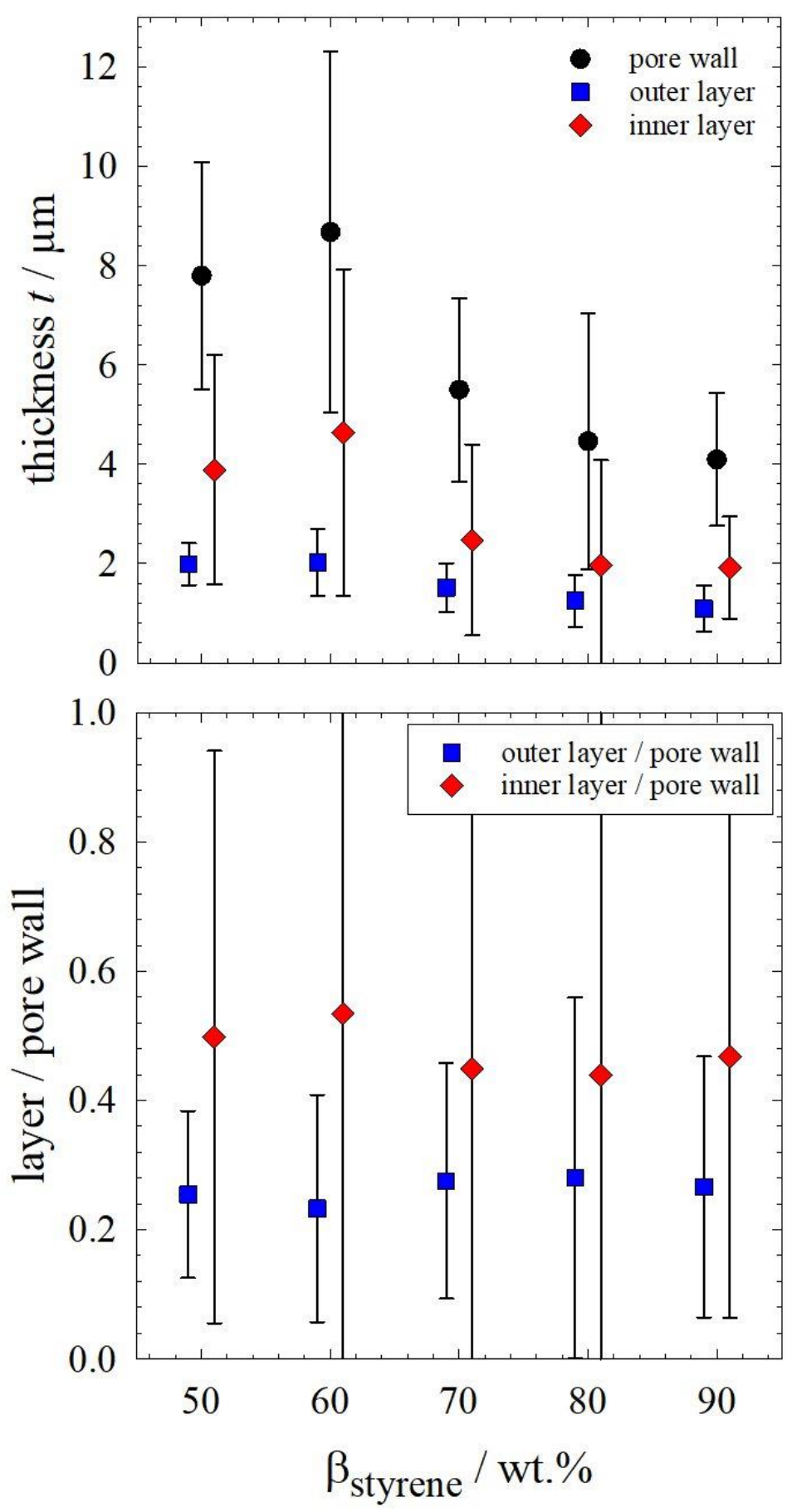

Figure 3.7: (top) Average thickness of the whole pore wall $t_{\text {pore wall (black circles), of the outer }}$

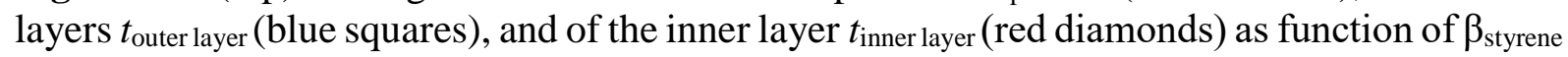
for macroporous PS/poly-DVB at $\beta_{\mathrm{KPS}}=2.98 \mathrm{wt} . \%$ and $\beta_{\text {surfactant }}=10 \mathrm{wt} . \%$. (bottom) Ratio $t_{\text {outer }}$ layer to $t_{\text {pore wall }}$ (blue squares) and ratio $t_{\text {inner layer }}$ to $t_{\text {pore wall }}$ (red diamonds) as function of $\beta_{\text {styrene. }}$. Since two outer layers exist, the two ratios in the bottom graph do not add up to 1 . For the sake of clarity, the blue squares and the red diamonds are shifted to lower and higher $\beta_{\text {styrene, }}$ respectively.

Again, the results are not in line with what was to be expected if osmotic transport of DVB was responsible for the transformation from spherical droplets to polyhedral pores. Even though 
$t_{\text {outer layer }}$ decreases as function of $\beta_{\text {styrene }}$ (as was expected), the same trend is observable for $t_{\text {inner }}$ layer and $t_{\text {pore wall. }}$. Thus, the fraction of the outer layer and the fraction of the inner layer remain

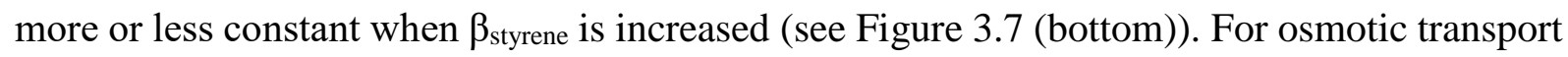
of DVB to be the correct hypothesis, the fraction of the outer layer should have decreased and the fraction of the inner layer should have increased. Overall, this means that the development of the layer thicknesses or their fractions, respectively, as function of $\beta_{\text {styrene }}$ serves as a second counterevidence to the hypothesis of osmotic transport of DVB. Note that in Figure 3.7 (bottom), the two fractions do not add up to 1. The reason for this can be explained with the help of Figure 3.6. Every time $t_{\text {pore wall }}$ and $t_{\text {inner layer }}$ are determined once, $t_{\text {outer layer }}$ is determined twice. However, in the end only one average $t_{\text {outer layer }}$ is calculated. Thus, $t_{\text {outer layer }}$ has to be

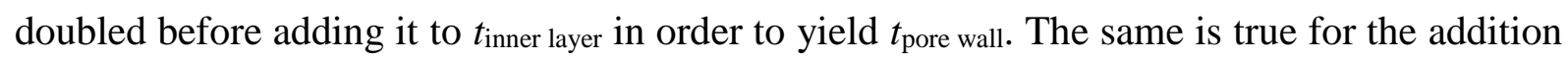
of the fraction of the outer layer to the fraction of the inner layer to end up at 1. Strikingly, the standard deviations of $t_{\text {pore wall }}$ and $t_{\text {inner layer }}$ are much larger than the standard deviation of $t_{\text {outer }}$ layer (see Figure 3.7 (top)). The same can be said about the large standard deviation of the fraction of the inner layer and the small standard deviation of the fraction of the outer layer. (see Figure 3.7 (bottom)). Again, the reason for this can be found in Figure 3.6 when looking at the thicknesses close to the struts ${ }^{5}$ (see Figure 3.6 (left)) and at the thicknesses in the films (see

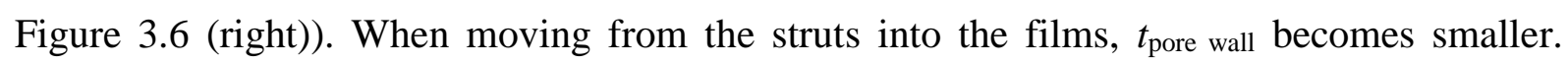
However, $t_{\text {pore wall }}$ was determined at several positions that were close to the struts, in the films, or between these two (see Appendix Section 6.2 (b), Figure 6.2 (right)). Therefore, $t_{\text {pore wall }}$ has a relatively high standard deviation. Looking at the development of $t_{\text {outer layer }}$ and $t_{\text {inner layer }}$ between the struts and the films, it becomes apparent that $t_{\text {outer layer remains roughly the same }}$ value independent of the location where it is measured. In contrast, and $t_{\text {inner layer changes from }}$ larger to smaller values when $t_{\text {pore wall }}$ changes from larger (close to struts) to smaller values

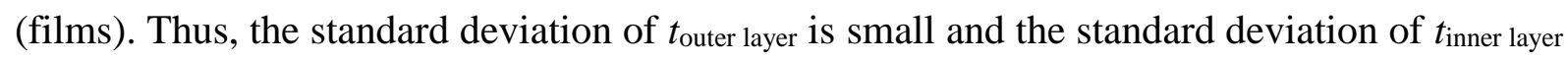
is large. These trends continue for the fraction of the outer layer and the fraction of the inner layer in Figure 3.7 (bottom).

When combining the trends from Figure 3.5 (right) and Figure 3.7 (top), it is noticeable that the overall surface of the pores increases as function of $\beta_{\text {styrene, while }} t_{\text {pore wall, }}, t_{\text {outer layer, and }} t_{\text {inner layer }}$ decrease as function of $\beta_{\text {styrene. Indeed, this makes sense: since the amount of available polymer }}$ is finite and the same independent of $\beta_{\text {styrene, }} t_{\text {pore wall }}, t_{\text {outer layer, }}$ and $t_{\text {inner layer }}$ have to decrease in order for the surface area to be able to increase. In other words, $t_{\text {pore wall, }} t_{\text {outer layer, }}$ and $t_{\text {inner layer }}$

\footnotetext{
${ }^{5}$ A strut corresponds to a former Plateau border which is now solidified.
} 
are spread out over a larger area. This relation can be expressed via a formula. For this purpose,

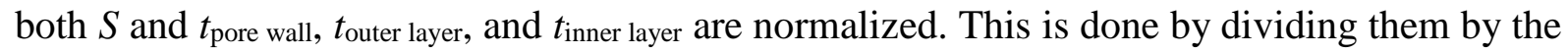

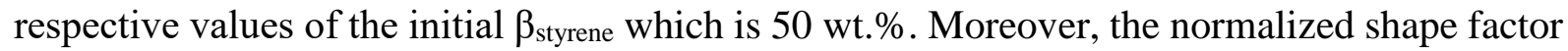
$S / S_{50}$ has to be squared since the key factor, the available volume of polymer, is a product of $t_{\text {pore wall }}, t_{\text {outer layer, }}$ or $t_{\text {inner layer }}$ and the surface area of a pores. The latter term is a quadratically scaling parameter that is determined from $3 \mathrm{D}$. In contrast, $S / S_{50}$ scales linearly and is determined from $2 \mathrm{D}$. By squaring $S / S_{50}$, this problem is easily solved and the desired relation is achieved. The product of $t / t_{50}$ and $\left(S / S_{50}\right)^{2}$ as function of $\beta_{\text {styrene }}$ is depicted for the pore wall (black circles), for the outer layer (blue squares), and for the inner layer (red diamonds) in Figure 3.8.

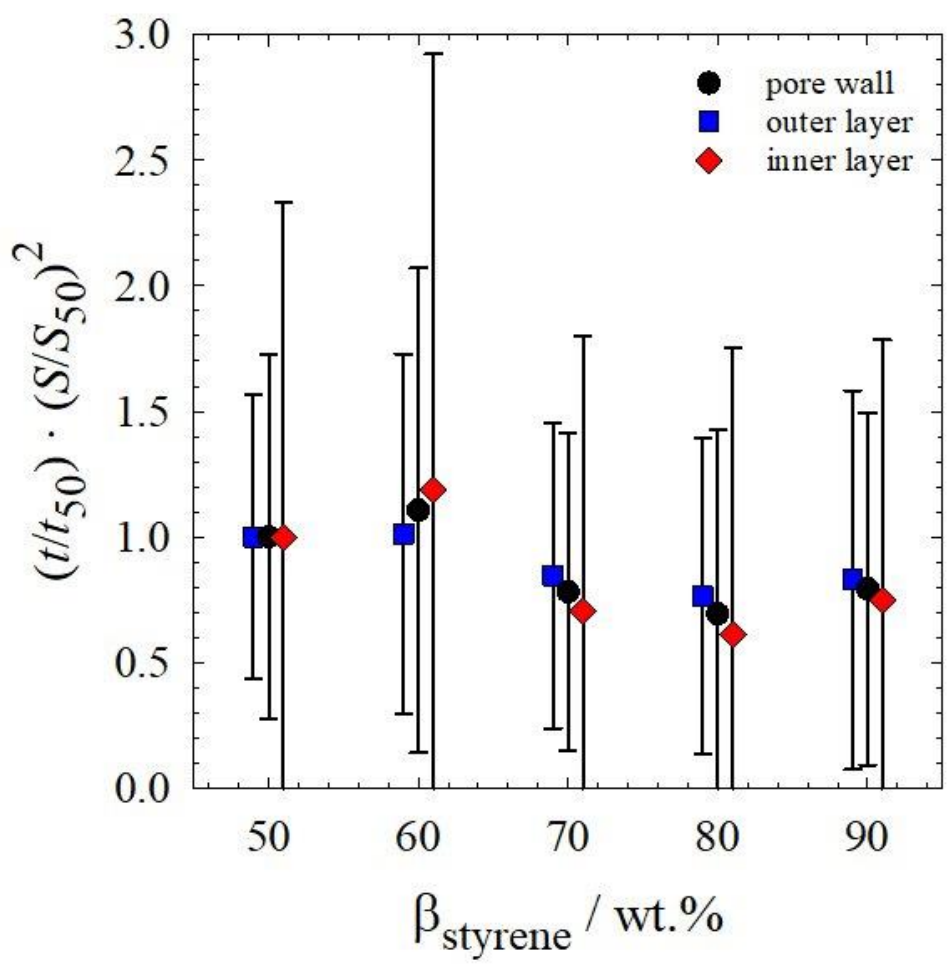

Figure 3.8: Normalized average thickness of the whole pore wall $t_{\text {pore wall }} / t_{\text {pore wall, } 50 \mathrm{wt} . \% \text { styrene }}$

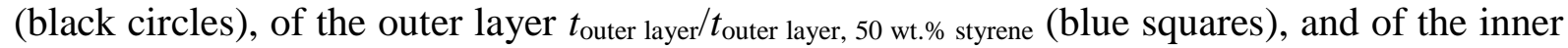
layer $t_{\text {inner layer }} / t_{\text {inner layer, } 50 \mathrm{wt.} \%}$ styrene (red diamonds) multiplied with the squared normalized shape

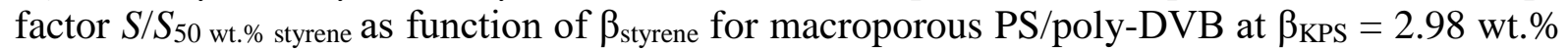
and $\beta_{\text {surfactant }}=10 \mathrm{wt} . \%$. For the sake of clarity, the blue squares and the red diamonds are shifted to lower and higher $\beta_{\text {styrene, respectively. }}$

Summed up, Figure 3.8 indeed shows that when $\beta_{\text {styrene }}$ is increased, the increase in surface area

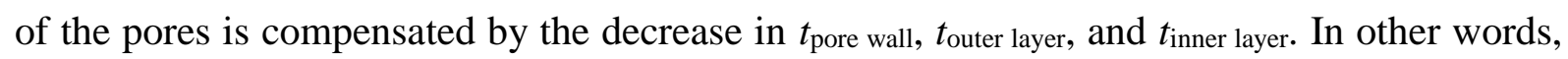
the total volume of the pore wall, of the outer layer, and of the inner layer remain constant, but they are spread out over a larger surface area. 


\subsubsection{Variation of KPS Mass Fraction $\beta \mathrm{KPS}$}

In Section 3.1.2, the KPS mass fraction $\beta_{\text {KPS }}$ of the water-in-styrene/DVB HIPEs was varied from 0.37 wt. $\%$ to 4.96 wt. $\%$. The first (0.37 wt.\%), second (0.75 wt.\%), and third (1.49 wt.\%) values of $\beta_{\mathrm{KPS}}$ are one eight, one fourth, and one half of the initial $\beta_{\mathrm{KPS}}$ of $2.98 \mathrm{wt} . \%$. The fifth $\beta_{\mathrm{KPS}}$ represents the maximum solubility of KPS in $\mathrm{H}_{2} \mathrm{O}$ [www2]. $\beta_{\text {styrene }}$ was kept constant at 50 wt. $\%$ and $\beta_{\text {surfactant }}$ was kept constant at $10 \mathrm{wt} . \%$. For a better overview, the corresponding mass fractions are listed in Table 3.2.

Table 3.2: Type of initiator, $\beta_{\text {styrene, }} \beta_{\text {initiator, }}$ and $\beta_{\text {surfactant }}$ of the samples. $\beta_{\text {initiator }}$ was varied, while $\beta_{\text {styrene }}$ and $\beta_{\text {surfactant }}$ were kept constant.

\begin{tabular}{|c|c|c|c|}
\hline Type of Initiator & $\boldsymbol{\beta}_{\text {styrene }} / \mathbf{w t}$ \% & $\boldsymbol{\beta}_{\text {initiator }}$ / wt.\% & $\boldsymbol{\beta}_{\text {surfactant }}$ / wt.\% \\
\hline water-soluble KPS & 50 & $\mathbf{0 . 3 7}$ & 10 \\
\hline water-soluble KPS & 50 & $\mathbf{0 . 7 5}$ & 10 \\
\hline water-soluble KPS & 50 & $\mathbf{1 . 4 9}$ & 10 \\
\hline water-soluble KPS & 50 & $\mathbf{2 . 9 8}$ & 10 \\
\hline water-soluble KPS & 50 & $\mathbf{4 . 9 6}$ & 10 \\
\hline monomer-soluble AIBN & 50 & $\mathbf{0 . 2 3}$ & 10 \\
\hline monomer-soluble AIBN & 50 & $\mathbf{0 . 4 5}$ & 10 \\
\hline monomer-soluble AIBN & 50 & $\mathbf{0 . 9 1}$ & 10 \\
\hline monomer-soluble AIBN & 50 & $\mathbf{1 . 8 2}$ & 10 \\
\hline
\end{tabular}

As in Section 3.1.1, samples were also prepared with the monomer-soluble initiator AIBN. However, now the AIBN mass fraction $\beta_{\text {AIBN }}$ was varied between $0.23-1.82 \mathrm{wt} . \%$. Note that for unknown reasons, it was not possible to produce a monodisperse water-in-styrene/DVB HIPE at $\beta_{\text {AIBN }}=3.02 \mathrm{wt} . \%$. Therefore, only four $\beta_{\text {AIBN values were studied. The way in which }}$ the samples were synthesized, polymerized, and processed can be found in the Experimental Section. Figure 3.9 depicts the SEM pictures of macroporous PS/polyDVB samples that were obtained from the polymerization of the monodisperse HIPEs listed in Table 3.2 with KPS as initiator. The magnifications are 100-fold (left column), 500-fold (middle column), and 1000fold (right column). At first glance, the shape of the 2D pore cross-sections is always hexagonal

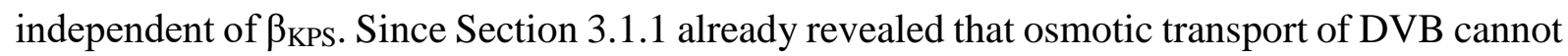
be the reason for the transformation from spherical droplets to polyhedral pores, it is not surprising that the expectations stated in the introduction of Section 3.1 did not come true. As a reminder, a higher $\beta_{\mathrm{KPS}}$ was supposed to lead to more circular 2D pore cross-sections since the polymerization would be sped up and osmotic transport of DVB would have less time to occur. When taking a closer look at the middle and right column of Figure 3.9, it is observable that the edges of the hexagons in the $2 \mathrm{D}$ pore cross-sections are nearly perfectly straight at low $\beta_{\mathrm{KPS}}$ and get more distorted at higher $\beta_{\mathrm{KPS}}$. 

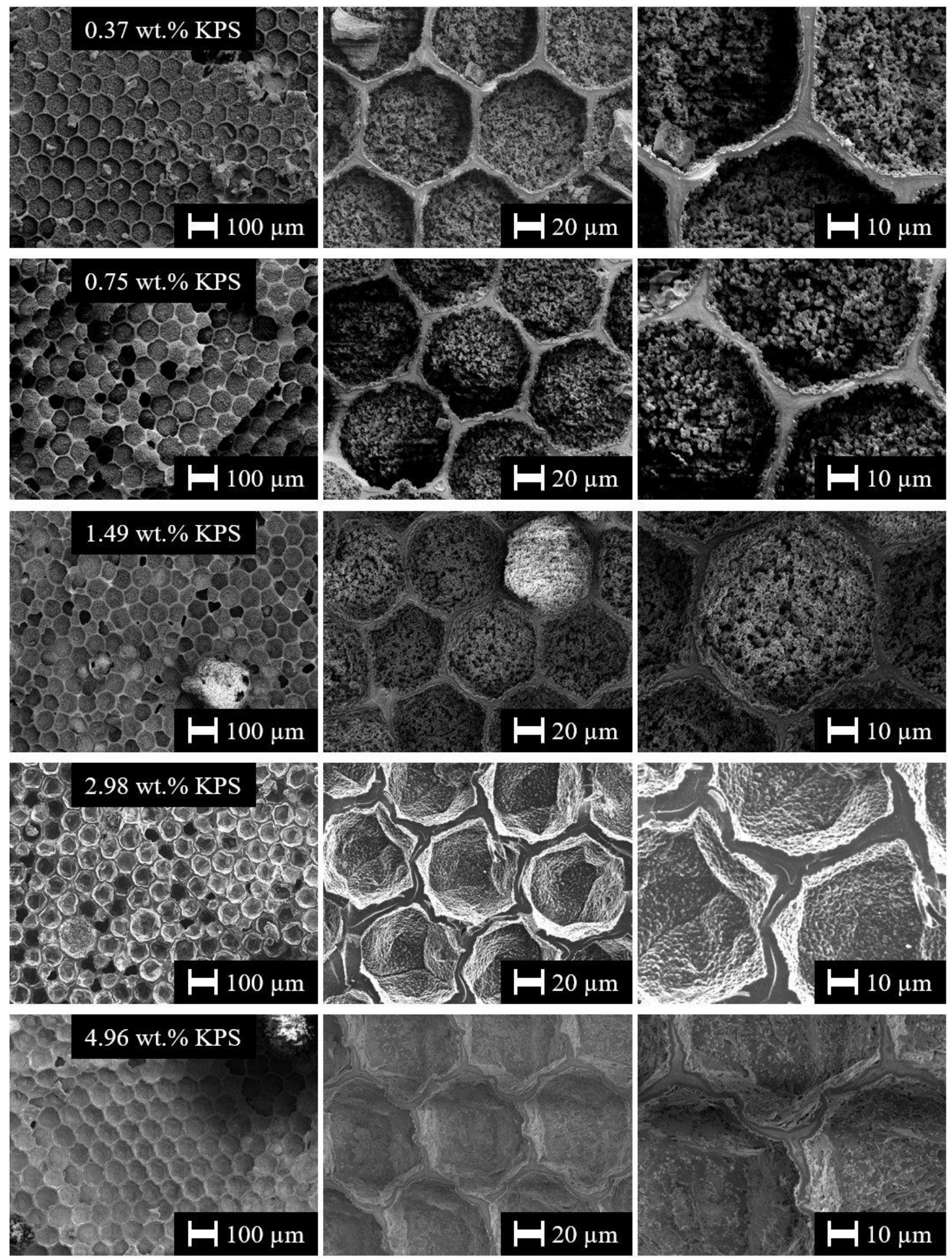

Figure 3.9: SEM pictures of macroporous PS/poly-DVB obtained via polymerization of monodisperse water-in-styrene/DVB HIPEs with $\beta_{\text {styrene }}=50 \mathrm{wt} . \%, \beta_{\text {surfactant }}=10 \mathrm{wt} . \%$, and $\beta_{\mathrm{KPS}}=0.37 \mathrm{wt} . \%,=0.75 \mathrm{wt} . \%,=1.49 \mathrm{wt} . \%,=2.98 \mathrm{wt} . \%$, and $=4.96 \mathrm{wt} . \%$. The magnifications are 100-fold (left column), 500-fold (middle column), and 1000-fold (right column). 
The diameter of the water droplets $d_{\text {droplet }}$ (black circles) before polymerization and the diameter of the pores $d_{\text {pore }}$ (pink squares) after polymerization as function of $\beta_{\text {KPS }}$ is depicted in Figure 3.10. Note that logarithmic plotting is used for the abscissa in Figure 3.10. The same will be true for the abscissae of Figure 3.12, Figure 3.13 (right), Figure 3.14 (top) and (bottom), and Figure 3.15.

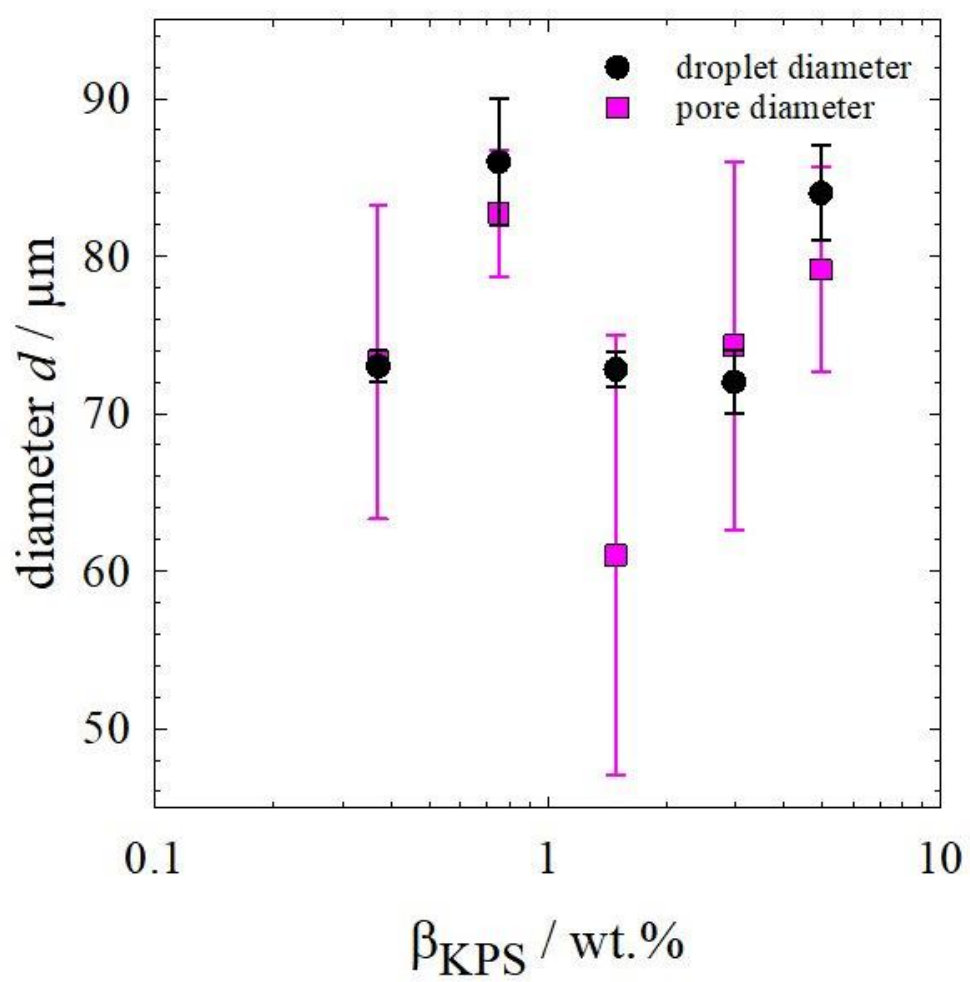

Figure 3.10: Average emulsion droplet diameter $d_{\text {droplet }}$ (black circles) and average pore diameter $d_{\text {pore }}$ (pink squares) as function of $\beta_{\text {KPS }}$ for macroporous PS/poly-DVB at $\beta_{\text {styrene }}=50$ wt. $\%$ and $\beta_{\text {surfactant }}=10 \mathrm{wt} . \%$.

Even though $d_{\text {droplet }}$ varies a bit more compared to Figure 3.2, the droplets were monodisperse. As before, polymerization leads to an increase of the error bars, but the pore cross-sections are still fairly monodisperse. Except for $\beta_{\mathrm{KPS}}=1.49 \mathrm{wt} . \%$ where $d_{\text {pore }}$ is $\sim 11 \mu \mathrm{m}$ smaller than $d_{\text {droplet }}$, the change from $d_{\text {droplet }}$ to $d_{\text {pore }}$ is minimal for the four other values of $\beta_{\text {KPS. }}$ As explained in Section 3.1.1, $d_{\text {pore }}$ was calculated from the area $A$ of the pore cross-sections (see equation (3.1)). Unfortunately, $\beta_{\text {KPS }}$ cannot be set to values higher than $\sim 5 \mathrm{wt}$ \% since this represents the maximum solubility of KPS in $\mathrm{H}_{2} \mathrm{O}$ [www2]. However, ammonium peroxodisulfate (APS) has a much higher solubility in $\mathrm{H}_{2} \mathrm{O}$ [www3] and is chemically very similar to KPS since only the counter-cation is different. Note that no experiments about were carried out and it was assumed that KPS and APS have similar reactivities. With APS as initiator, $\beta_{\text {APS }}$ was set to values 
between $0.32-39.44$ wt. $\%$. Here, $\beta_{\mathrm{APS}}=0.32$ wt. $\%,=0.63$ wt. $\%,=1.26$ wt. $\%,=2.52$ wt. $\%$, and $=4.20 \mathrm{wt} . \%$ are molarly equivalent to the five values of $\beta_{\mathrm{KPS}}$ in Figure 3.9. Additionally, samples with $\beta_{\mathrm{APS}}=9.86 \mathrm{wt} . \%,=19.72 \mathrm{wt} . \%$, and $=39.44 \mathrm{wt} . \%$ were produced. The SEM pictures of these eight samples are depicted in Figure 6.3 and Figure 6.4 in Appendix Section 6.2 (c). Without going into too much detail, it is apparent that for molarly equal mass fractions, the morphology is roughly the same if either KPS or APS is used. Up to $\beta_{\text {APS }}=9.86 \mathrm{wt} . \%$, the shape of the $2 \mathrm{D}$ pore cross-sections can roughly be described as hexagonal. With a further in-
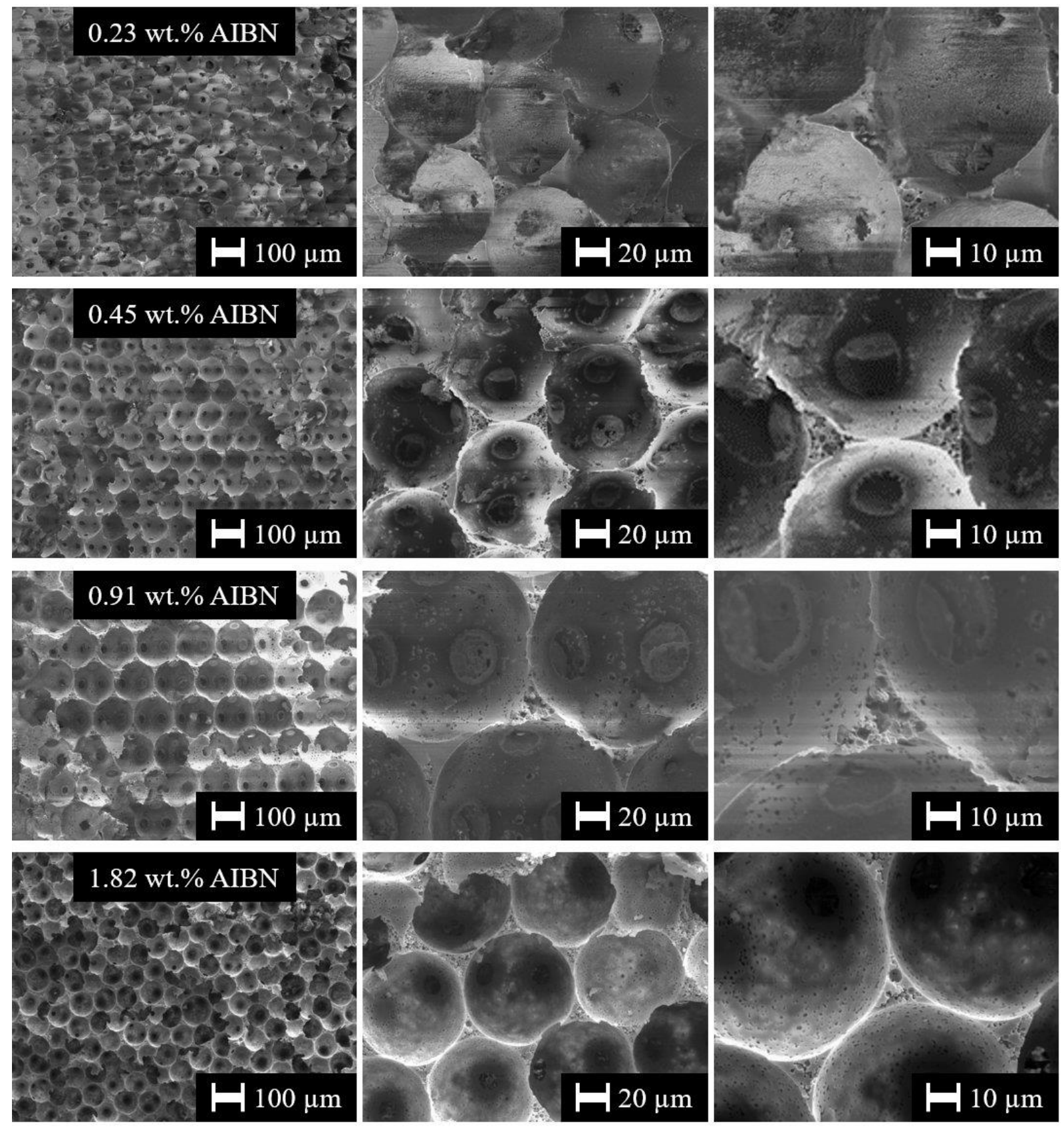

Figure 3.11: SEM pictures of macroporous PS/poly-DVB obtained via polymerization of monodisperse water-in-styrene/DVB HIPEs with $\beta_{\text {styrene }}=50 \mathrm{wt} . \%, \beta_{\text {surfactant }}=10 \mathrm{wt} . \%$, and $\beta_{\text {AIBN }}=0.23 \mathrm{wt} . \%,=0.45 \mathrm{wt} . \%,=0.91 \mathrm{wt} . \%$, and $=1.82 \mathrm{wt} . \%$. The magnifications are 100fold (left column), 500-fold (middle column), and 1000-fold (right column). 
crease of $\beta_{\mathrm{APS}}$, the pores become more and more disordered as well as polydisperse. At the highest value for $\beta_{\mathrm{APS}}=39.44 \mathrm{wt} . \%$, the interconnectivity changes from closed-pore to openpore. Note that such high initiator mass fractions are far from any reasonable field of application and were only used to test the limits of the system.

When $\beta_{\text {AIBN }}$ is varied, the shape of the pore cross-sections is always circular. This is depicted in Figure 3.11 where the magnifications were 100-fold (left column), 500-fold (middle column), and 1000-fold (right column). The corresponding mass fractions were listed in Table 3.2. As in Figure 3.4 where the morphology was largely independent of $\beta_{\text {styrene, }}$ it is also independent of $\beta_{\text {AIBN }}$. However, looking at the diameters of the pore cross-sections in Figure 3.11, it is obvious that at $\beta_{\mathrm{AIBN}}=0.91 \mathrm{wt} . \% d_{\text {pore }}$ is much larger than for the three other values of $\beta_{\mathrm{AIBN}}$. This can also be seen in Figure 3.12 where $d_{\text {droplet }}$ (black circles) before polymerization and $d_{\text {pore }}$ (pink squares) after polymerization are depicted as function of $\beta_{\text {AIBN }}$.

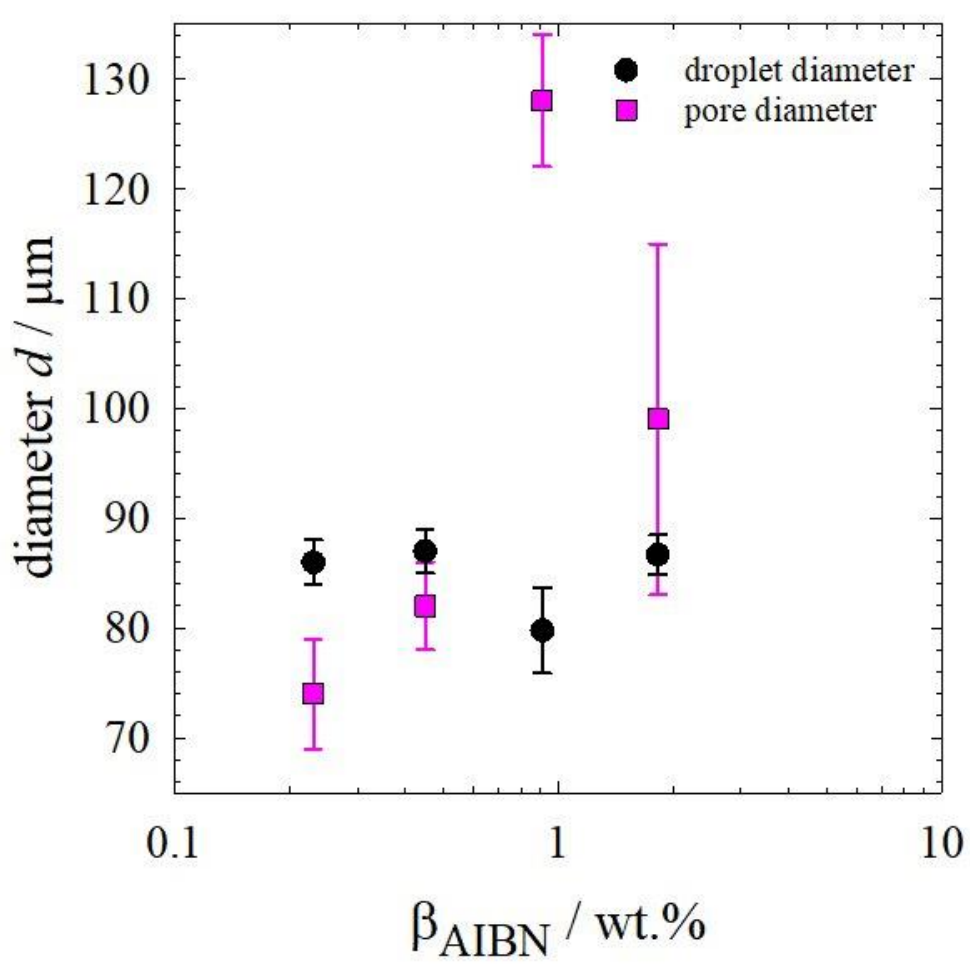

Figure 3.12: Average emulsion droplet diameter $d_{\text {droplet }}$ (black circles) and average pore diameter $d_{\text {pore }}$ (pink squares) as function of $\beta_{\mathrm{AIBN}}$ for macroporous PS/poly-DVB at $\beta_{\text {styrene }}=50$ wt. $\%$ and $\beta_{\text {surfactant }}=10 \mathrm{wt} . \%$.

For $\beta_{\mathrm{AIBN}}=0.23 \mathrm{wt} . \%$ and $\beta_{\mathrm{AIBN}}=0.45 \mathrm{wt} . \%$, the high monodispersity of the droplets is largely conserved in the pores, while $d_{\text {pore }}$ is slightly smaller than $d_{\text {droplet. }}$ For $\beta_{\text {AIBN }}=0.91 \mathrm{wt} . \%$, the high monodispersity is also mostly retained, while $d_{\text {pore }}$ increases tremendously compared to 
$d_{\text {droplet. }}$ Finally, for $\beta_{\text {AIBN }}=1.82 \mathrm{wt} . \%$, the pores did not maintain the high monodispersity of the droplets, though $d_{\text {pore }}$ is closer to $d_{\text {droplet }}$ compared to $\beta_{\text {AIBN }}=0.91 \mathrm{wt} . \%$. Overall, it appears as if the transition from HIPE to macroporous PS/polyDVB gets more susceptible to changes when $\beta_{\text {AIBN }}$ is increased. This is further supported by the fact that it was not possible to produce a sample with $\beta_{\mathrm{AIBN}}=3.02 \mathrm{wt}$. \% where the droplets were already polydisperse when they were generated in the microfluidic chip. Nevertheless, Figure 3.11 shows that $\beta_{\text {AIBN }}$ has at least no influence on the shape of the pore cross-sections and on the interconnectivity of the pores.

\section{Shape of Pore Cross-Sections: Shape Factor $S$}

Looking again at Figure 3.9, one sees that the edges of the hexagons in the 2D pore crosssections are nearly perfectly straight for small values of $\beta_{\text {KPS }}$ and get more distorted at higher values of $\beta_{\text {KPS. }}{ }^{6}$ Therefore, the shape factor $S$ should be very close to 1.05 for small $\beta_{\text {KPS }}$ and somewhat higher for larger $\beta_{\mathrm{KPS}}$ which is indeed the case as can be seen in Figure 3.13 (right). As a reminder, Figure 3.13 (left) shows values of $S$ for a distorted hexagon, for a hexagon, and for a circle.

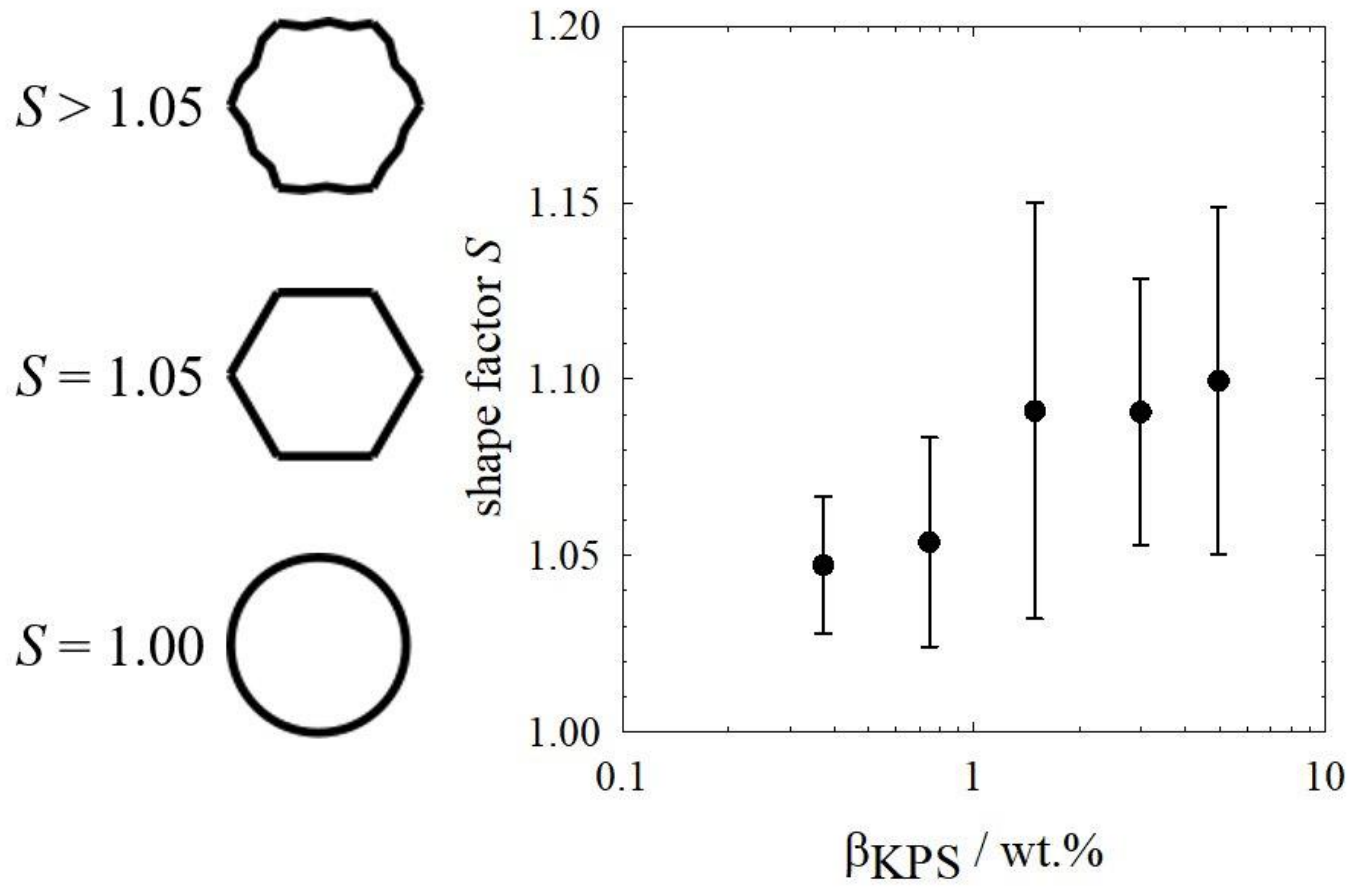

Figure 3.13: (left) Exemplary values of the shape factor $S$ for a sphere (bottom), a hexagon (middle), and a distorted hexagon (top). (right) $S$ as function of $\beta_{\mathrm{KPS}}$ for macroporous PS/polyDVB at $\beta_{\text {styrene }}=50 \mathrm{wt} . \%$ and $\beta_{\text {surfactant }}=10 \mathrm{wt} . \%$.

\footnotetext{
${ }^{6}$ The same is true for APS as initiator.
} 
When $\beta_{\mathrm{KPS}}=0.37 \mathrm{wt} . \%$ and $\beta_{\mathrm{KPS}}=0.75 \mathrm{wt} . \%, S$ is exactly 1.05 as was expected for the hexagonal shape with nearly perfectly straight edges. In contrast, for $\beta_{\mathrm{KPS}}=1.49 \mathrm{wt} \%,=2.98$ wt. $\%$, and $=4.96$ wt. $\%, S$ is around 1.10 as was already the case for the hexagonal pore crosssections in Figure 3.1 and Figure 3.5 (right). Again, the reason for this increase is that changing the edges from straight to distorted results in an increase of $C$ without simultaneously increasing $A$ which, in turn, increases $S$ from 1.05 to 1.10 .

\section{Thickness of Pore Wall and Layers}

Next, the development of the thickness of the pore wall $t_{\text {pore wall, }}$ of the thickness of the outer

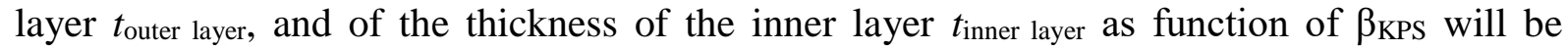
investigated. Again, the development of the fraction of the outer layer ( $\left.t_{\text {outer layer }} / t_{\text {pore wall }}\right)$ and of the fraction of the inner layer $\left(t_{\text {inner layer }} / t_{\text {pore wall }}\right)$ as function of $\beta_{\text {KPS }}$ will be examined as well. Looking at Figure 3.9, one sees that the three-layered structure of the pore walls is independent of $\beta_{\mathrm{KPS}}$. As a reminder, an increase of $\beta_{\mathrm{KPS}}-$ or $\beta_{\mathrm{APS}}-$ was supposed to diminish the differences between outer layer and inner layer when assuming an osmotic transport of DVB. Figure 3.14 (top) depicts the development of $t_{\text {pore wall }}$ (black circles), $t_{\text {outer layer }}$ (blue squares), and $t_{\text {inner layer }}$

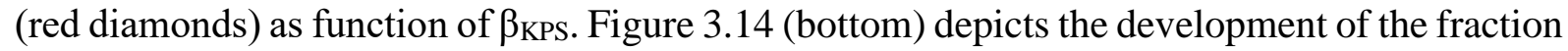
of the outer layer (blue squares) and of the fraction of the inner layer (red diamonds) as function

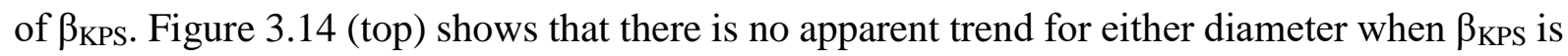
varied. Except for $\beta_{\mathrm{KPS}}=2.98 \mathrm{wt}$. $\%$ where $t_{\text {pore wall, }} t_{\text {outer layer, }}$ and $t_{\text {inner layer }}$ are larger than for the remaining four values of $\beta_{\mathrm{KPS}}$, the thicknesses are more or less constant. Therefore, it is no surprise that in Figure 3.14 (bottom), the fraction of the outer layer and the fraction of the inner layer are constant and independent of $\beta_{\mathrm{KPS}}$. As a reminder, the two fractions in Figure 3.14 (bottom) do not add up to 1 because for this to happen, the fraction of the outer layer has to be doubled (see Section 3.1.1, Figure 3.6). The reason for why the values of the standard deviation are much smaller for $t_{\text {outer layer }}$ and for the fraction of the outer layer compared to $t_{\text {pore }}$ wall, to $t_{\text {inner }}$ layer and to the fraction of the inner layer was also already discussed in Section 3.1.1 with the help of Figure 3.6. Finally, a multiplication of the normalized values of $t_{\text {pore wall }}$, of $t_{\text {outer layer, }}$ and

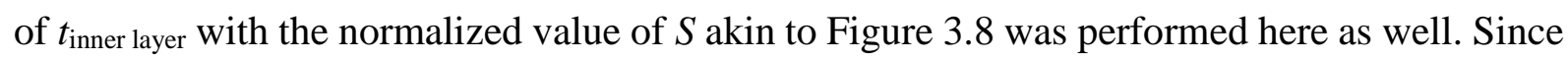
the initial $\beta_{\text {KPS }}$ was $2.98 \mathrm{wt} . \%, t_{\text {pore wall }}, t_{\text {outer layer, }} t_{\text {inner layer, and }} S$ of this $\beta_{\text {KPs }}$ were chosen as the values the other four respective values were normalized to. The product of $t / t_{2.98}$ and $\left(S / S_{2.98}\right)^{2}$ for the pore wall (black circles), for the outer layer (blue squares), and for the inner layer (red diamonds) is plotted in Figure 3.15 as function of $\beta_{\mathrm{KPS}}$. 

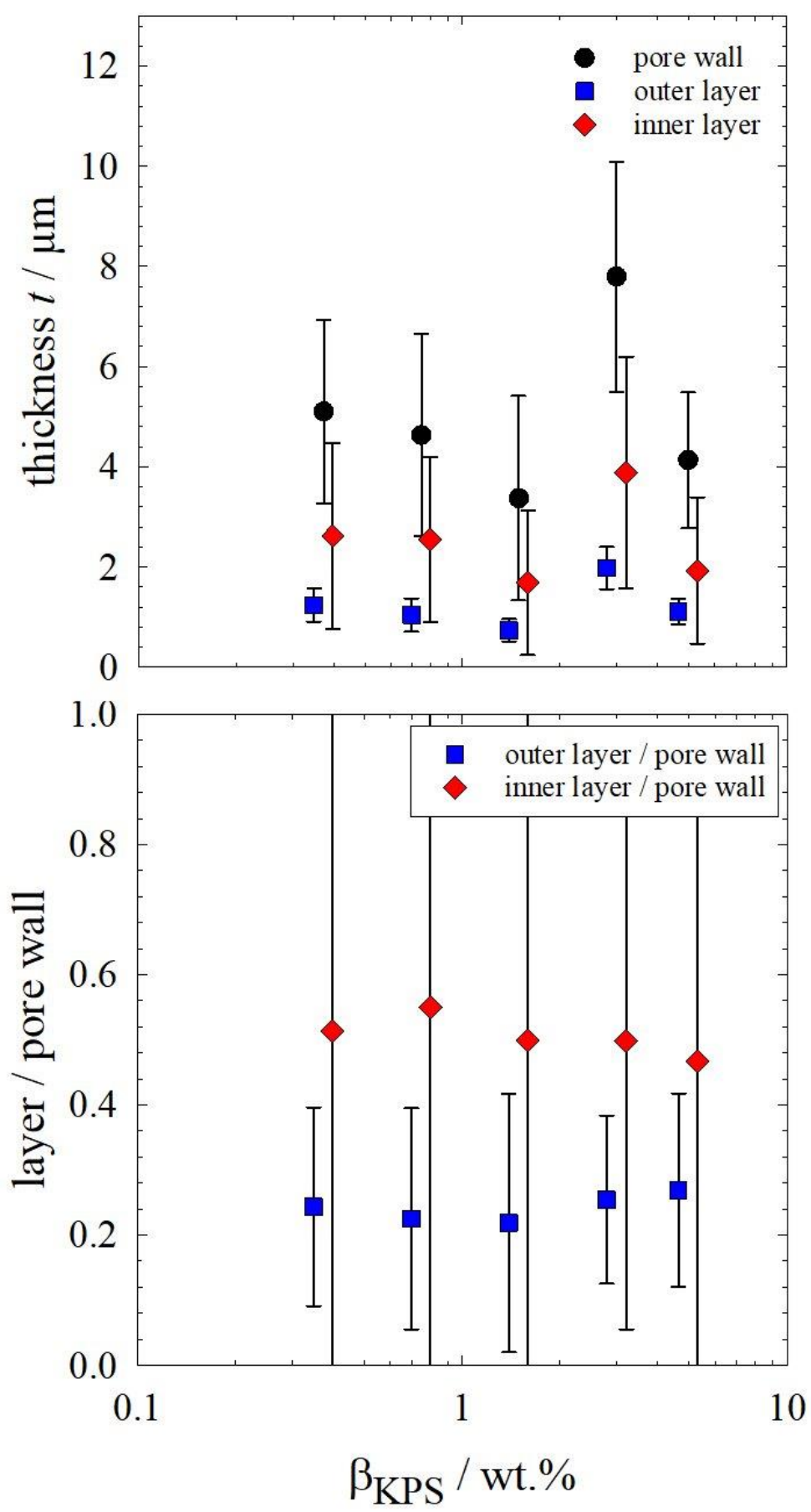

Figure 3.14: (top) Average thickness of the whole pore wall $t_{\text {pore wall }}$ (black circles), of the outer

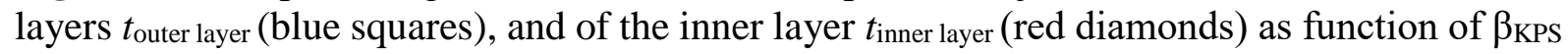
for macroporous PS/poly-DVB at $\beta_{\text {styrene }}=50 \mathrm{wt} . \%$ and $\beta_{\text {surfactant }}=10 \mathrm{wt} . \%$. (bottom) Ratio $t_{\text {outer }}$

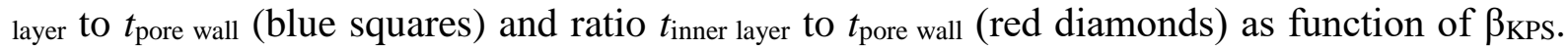
Since two outer layers exist, the two ratios in the bottom graph do not add up to 1 . For the sake of clarity, the blue squares and the red diamonds are shifted to lower and higher $\beta_{\mathrm{KPS}}$, respectively.

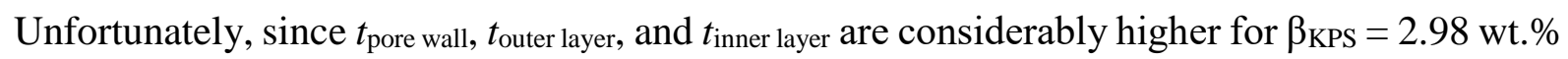
compared to the other values of $\beta_{\mathrm{KPS}}$ (see Figure 3.14 (top)), the product of $t / t_{2.98}$ and $\left(S / S_{2.98}\right)^{2}$ 


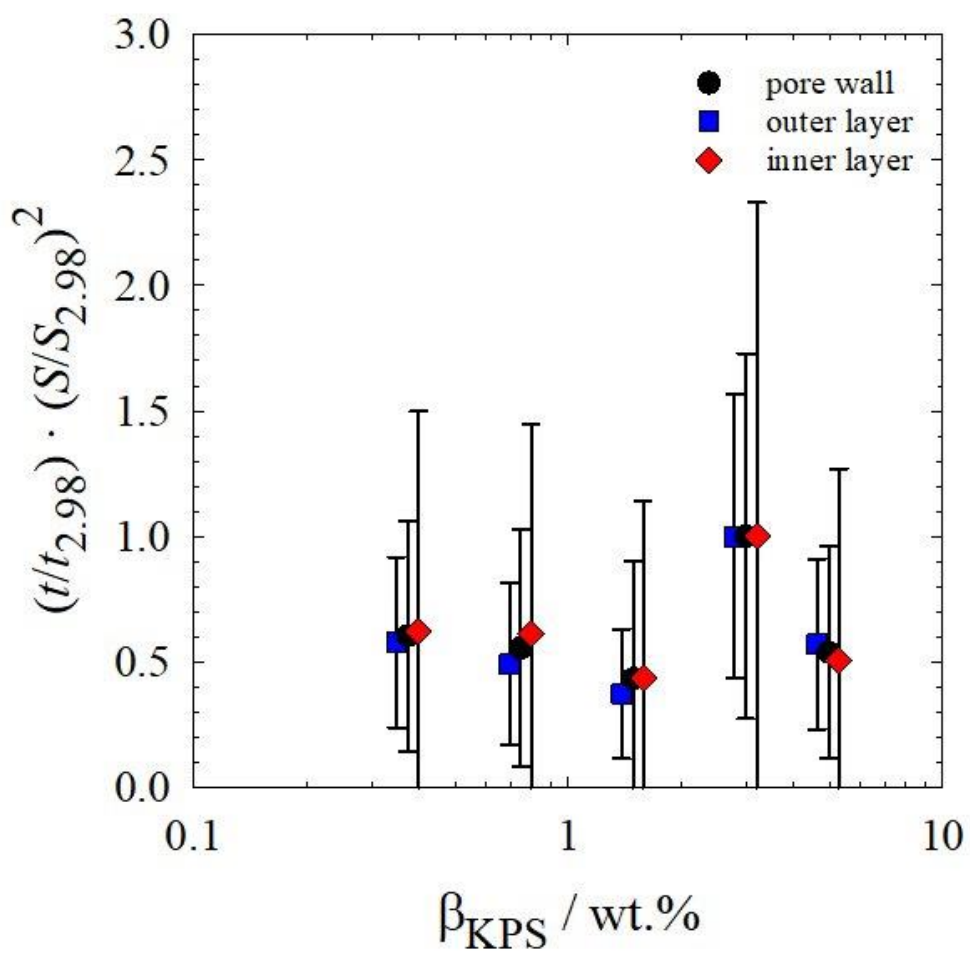

Figure 3.15: Normalized average diameter of the whole pore wall $t_{\text {pore wall }} / t_{\text {pore wall, } 2.98 \text { wt.\% KPS }}$

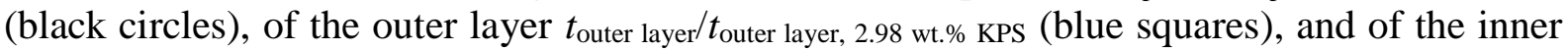

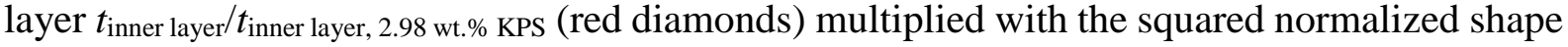

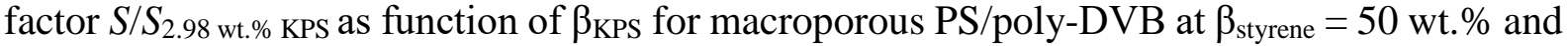
$\beta_{\text {surfactant }}=10 \mathrm{wt} . \%$. For the sake of clarity, the blue squares and the red diamonds are shifted to lower and higher $\beta_{\mathrm{KPS}}$, respectively.

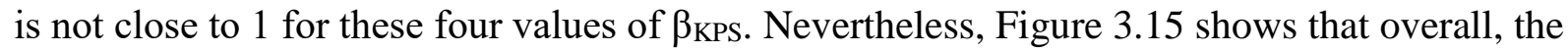
total volume of the pore wall, of the outer layer, and of the inner layer is constant. The reasons why the normalized shape factor $S / S_{2.98}$ has to be squared and why the error bars are this large was discussed in Section 3.1.1. 


\subsection{New Mechanism: Surfactant Diffusion}

\subsubsection{Experimental Results}

While Section 3.1 focused on disproving the osmotic transport of DVB, this Section is about developing and experimentally proving a new hypothesis. The results of this Section were also published in a paper in the Journal of Colloid and Interface Science [Koc21]. The influence of $\beta_{\text {styrene }}$ and $\beta_{\mathrm{KPS}}$ on the morphology of macroporous PS/polyDVB was investigated in the previous Section. Thus, Section 3.2 starts with the variation of a third parameter, namely $\beta_{\text {surfactant, }}$ which was changed from its initial value of $10 \mathrm{wt} . \%$ to $\beta_{\text {surfactant }}=5 \mathrm{wt} . \%,=15 \mathrm{wt} . \%$, and $=20 \mathrm{wt} . \%$. As a reminder, $\beta_{\text {surfactant }}$ is the surfactant mass divided by the combined mass of

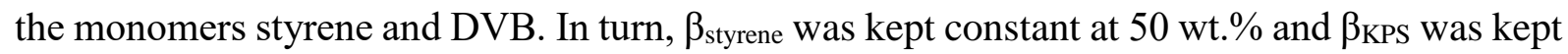
constant at $2.98 \mathrm{wt} . \%$. As before, the corresponding mass fractions are listed in Table 3.3.

Table 3.3: Type of initiator, $\beta_{\text {styrene, }} \beta_{\text {initiator, }}$ and $\beta_{\text {surfactant }}$ of the samples. $\beta_{\text {surfactant }}$ was varied, while $\beta_{\text {styrene }}$ and $\beta_{\text {initiator }}$ were kept constant.

\begin{tabular}{|c|c|c|c|}
\hline Type of Initiator & $\boldsymbol{\beta}_{\text {styrene }} / \mathbf{w t} . \%$ & $\boldsymbol{\beta}_{\text {initiator }} / \mathbf{w t} . \%$ & $\boldsymbol{\beta}_{\text {surfactant }} / \mathbf{w t}$.\% \\
\hline water-soluble KPS & 50 & 2.98 & $\mathbf{5}$ \\
\hline water-soluble KPS & 50 & 2.98 & $\mathbf{1 0}$ \\
\hline water-soluble KPS & 50 & 2.98 & $\mathbf{1 5}$ \\
\hline water-soluble KPS & 50 & 2.98 & $\mathbf{2 0}$ \\
\hline monomer-soluble AIBN & 50 & 1.82 & $\mathbf{5}$ \\
\hline monomer-soluble AIBN & 50 & 1.82 & $\mathbf{1 0}$ \\
\hline monomer-soluble AIBN & 50 & 1.82 & $\mathbf{1 5}$ \\
\hline monomer-soluble AIBN & 50 & 1.82 & $\mathbf{2 0}$ \\
\hline
\end{tabular}

As in Section 3.1, samples were also prepared with the monomer-soluble initiator AIBN. Table 3.3 gives an overview of the corresponding samples. The way in which the samples in Table 3.3 were synthesized, polymerized, and processed is described in the Experimental Section. Figure 3.16 depicts the SEM pictures of the KPS samples from Table 3.3 at a 100-fold (left column), a 500-fold (middle column), and a 1000-fold magnification (right column). Looking at the left column of Figure 3.16, it is obvious that the pores are close-packed and fairly monodisperse for all four values of $\beta_{\text {surfactant }}$ Additionally, the shape of the 2D pore crosssections is always hexagonal (see Figure 3.16 (middle column and right column)) and the pore walls are always made up of three layers (see Figure 3.16 (right column)). As a reminder, a hexagonal shape of the pore cross-sections means that the area of the water/monomer interface increases during polymerization. 

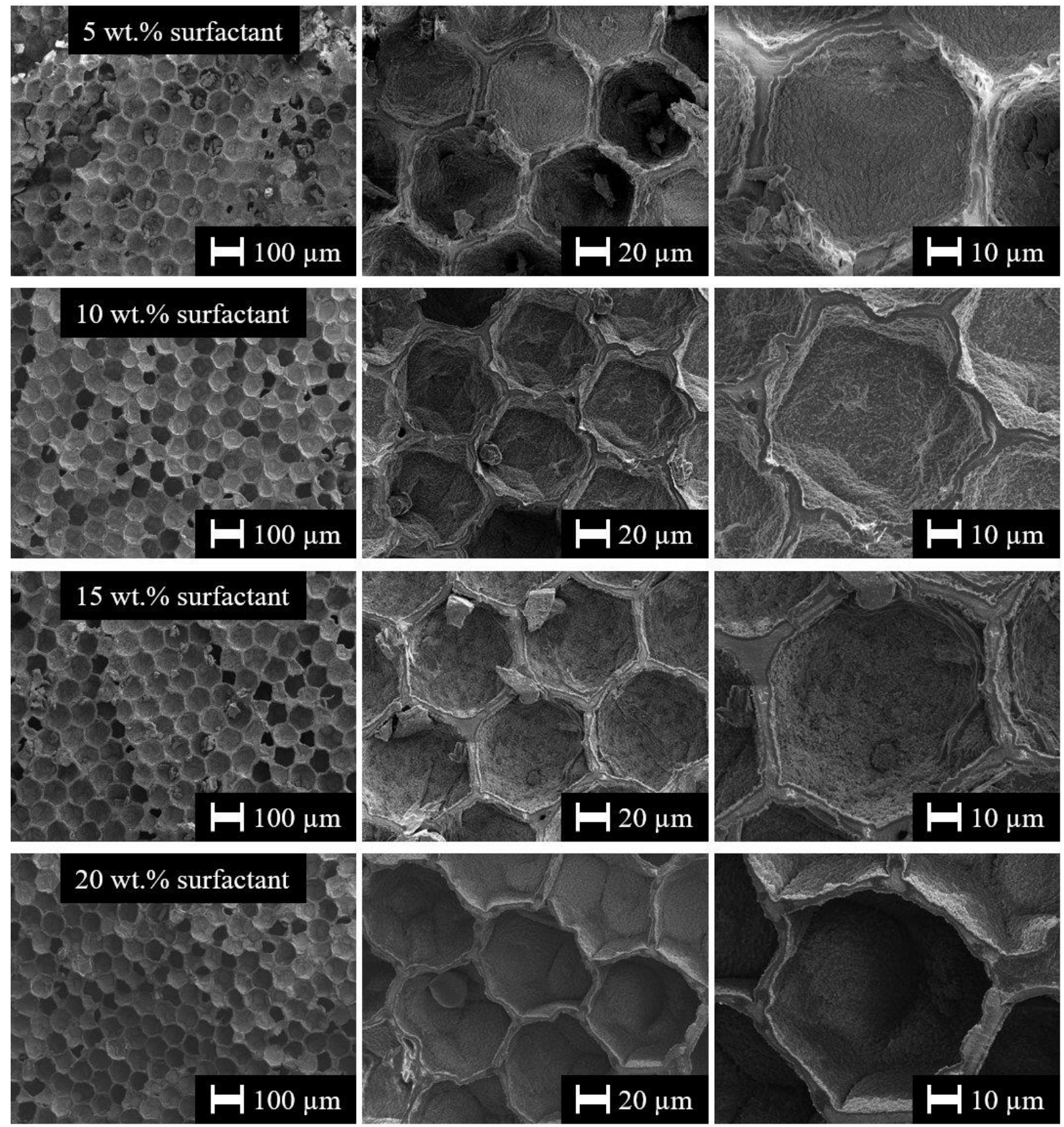

Figure 3.16: SEM pictures of macroporous PS/poly-DVB obtained via polymerization of monodisperse water-in-styrene/DVB HIPEs with $\beta_{\text {styrene }}=50$ wt. $\%, \beta_{\text {KPS }}=2.98$ wt. $\%$, and $\beta_{\text {surfactant }}=5 \mathrm{wt} . \%,=10 \mathrm{wt} . \%,=15 \mathrm{wt} . \%$, and $=20 \mathrm{wt} . \%$. The magnifications are 100 -fold (left column), 500-fold (middle column), and 1000-fold (right column).

As in Section 3.1, the average diameter of the water droplets $d_{\text {droplet }}$ before polymerization and the average diameter of the pores $d_{\text {pore }}$ after polymerization were determined. In Figure 3.17, $d_{\text {droplet }}$ (black circles) and $d_{\text {pore }}$ (pink squares) are plotted as function of $\beta_{\text {surfactant. Looking at }}$ $d_{\text {droplet}}$, one sees that highly monodisperse droplets with a uniform diameter between $71-73$ $\mu \mathrm{m}$ and with standard deviations of only $1-2 \mu \mathrm{m}$ were generated. After the polymerization, the polydispersity is slightly higher with a standard deviation for $d_{\text {pore }}$ of $3-4 \mu \mathrm{m}$. Thus, the 


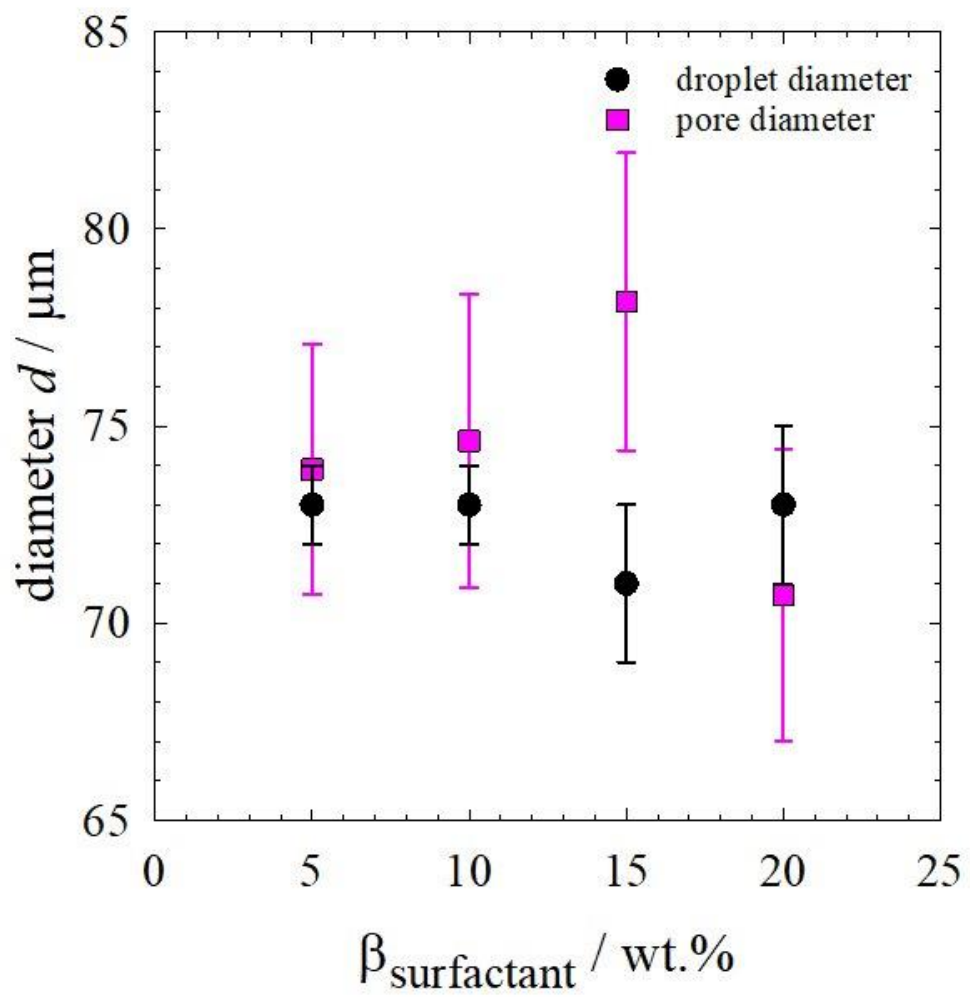

Figure 3.17: Average emulsion droplet diameter $d_{\text {droplet }}$ (black circles) and average pore diameter $d_{\text {pore }}$ (pink squares) as function of $\beta_{\text {surfactant }}$ for macroporous PS/poly-DVB at $\beta_{\text {styrene }}=$ $50 \mathrm{wt} . \%$ and $\beta_{\mathrm{KPS}}=2.98 \mathrm{wt} . \%$.

pores can still be regarded as highly monodisperse. The polymerization furthermore influences the transition from $d_{\text {droplet }}$ to $d_{\text {pore }}$ in that $d_{\text {pore }}$ is slightly higher than $d_{\text {droplet }}$ for $\beta_{\text {surfactant }}=5 \mathrm{wt} . \%$ and for $\beta_{\text {surfactant }}=10 \mathrm{wt} . \%$. For $\beta_{\text {surfactant }}=15 \mathrm{wt} . \%, d_{\text {pore }}$ is somewhat larger than $d_{\text {droplet }}$ and for $\beta_{\text {surfactant }}=20 \mathrm{wt} . \%, d_{\text {pore }}$ is slightly lower than $d_{\text {droplet. }}$. As a reminder, since $d_{\text {pore }}$ is calculated as if the pore cross-sections were circular, $d_{\text {pore }}$ always carries a small, systematic error. This means that the small differences between $d_{\text {droplet }}$ and $d_{\text {pore }}$ can be neglected.

The composition of the samples where KPS was substituted by AIBN are given in Table 3.3. The SEM pictures that were obtained from polymerizing and processing these samples are depicted in Figure 3.18. The magnifications were 100-fold (left column), 500-fold (middle column), and 1000-fold (right column). Looking at the left column of Figure 3.18, one sees that while the pores are close-packed and ordered for $\beta_{\text {surfactant }}=5 \mathrm{wt}$. $\%$, the packing density and the order of the pores decreases when $\beta_{\text {surfactant }}$ is increased. Nevertheless, the shape of the 2D pore cross-sections is always circular. Moreover, an open-pore morphology is found independent of

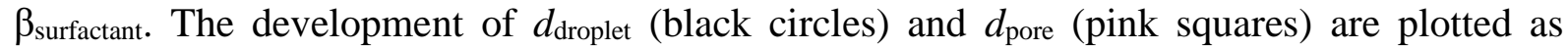
function of $\beta_{\text {surfactant }}$ in Figure 3.19. Again, it was possible to generate monodisperse water drop- 

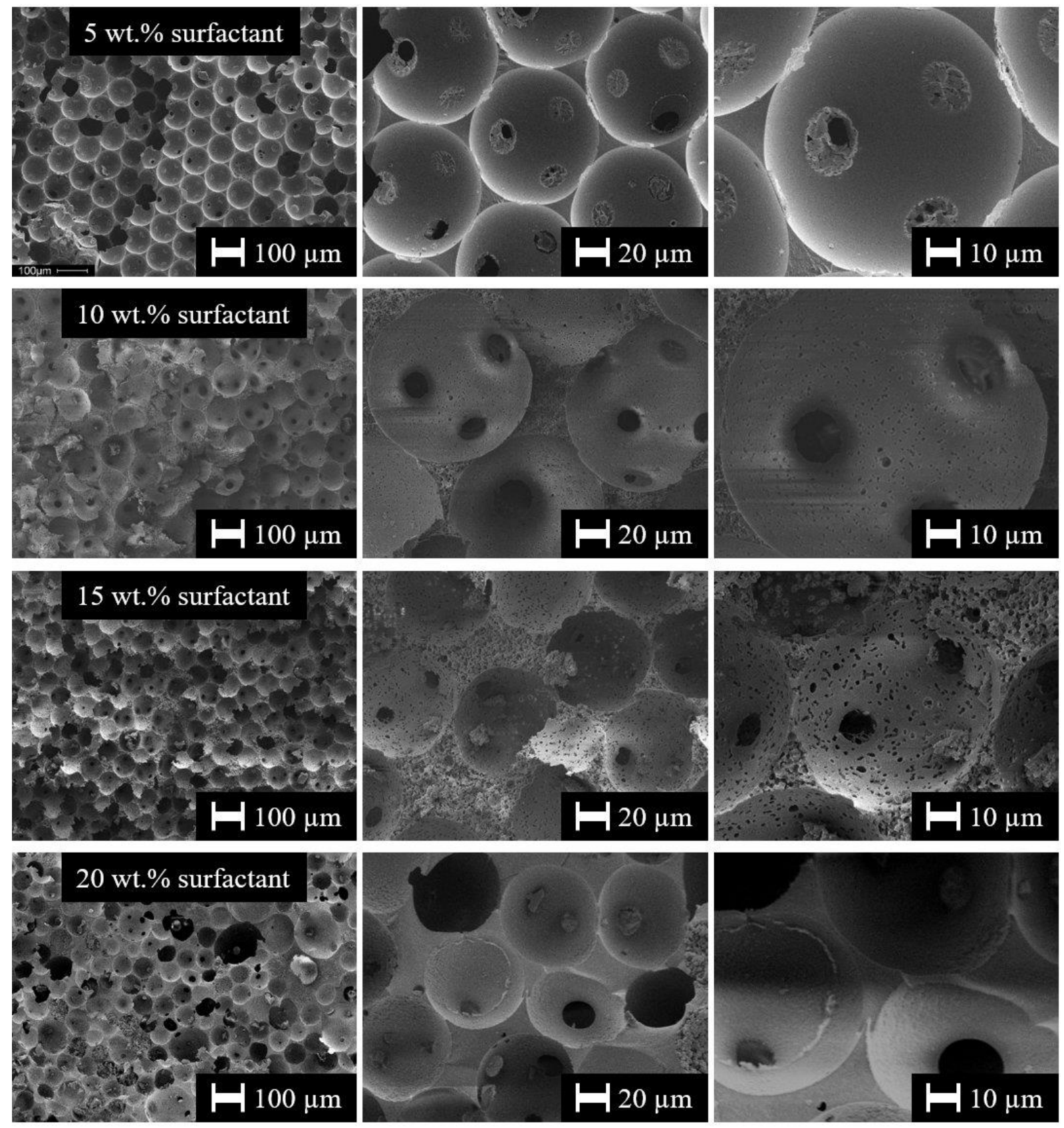

Figure 3.18: SEM pictures of macroporous PS/poly-DVB obtained via polymerization of monodisperse water-in-styrene/DVB HIPEs with $\beta_{\text {styrene }}=50 \mathrm{wt} . \%, \beta_{\text {AIBN }}=1.82 \mathrm{wt} . \%$, and $\beta_{\text {surfactant }}=5 \mathrm{wt} . \%,=10 \mathrm{wt} . \%,=15 \mathrm{wt} . \%$, and $=20 \mathrm{wt} . \%$. The magnifications are 100 -fold (left column), 500-fold (middle column), and 1000-fold (right column).

lets with microfluidics. The values of $d_{\text {droplet }}$ lie between $72-77 \mu \mathrm{m}$ and the droplets were fairly monodisperse with standard deviations of $2-5 \mu \mathrm{m}$. For $\beta_{\text {surfactant }}=15 \mathrm{wt} . \%$ and $\beta_{\text {surfactant }}=20$ wt.\%, polymerization changed neither the diameter nor the standard deviation in a significant way. However, note that very large pores were excluded from the determination of $d_{\text {pore }}$ at $\beta_{\text {surfactant }}=20 \mathrm{wt} . \%$. If these very large pores were to be included, $d_{\text {pore }}$ and especially the stan- 


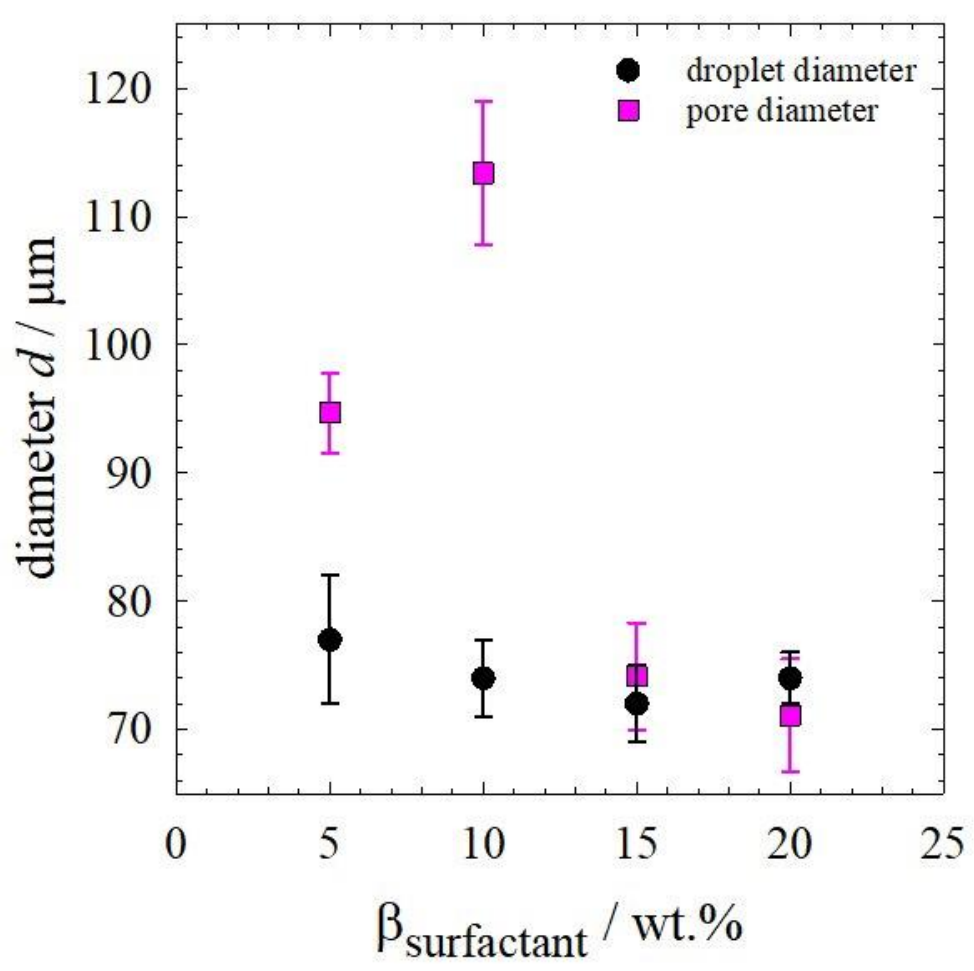

Figure 3.19: Average emulsion droplet diameter $d_{\text {droplet }}$ (black circles) and average pore diameter $d_{\text {pore }}$ (pink squares) as function of $\beta_{\text {surfactant }}$ for macroporous PS/poly-DVB at $\beta_{\text {styrene }}=$ $50 \mathrm{wt} . \%$ and $\beta_{\mathrm{AIBN}}=1.82 \mathrm{wt} . \%$.

dard deviation would increase significantly. For $\beta_{\text {surfactant }}=5 \mathrm{wt} . \%$ and $\beta_{\text {surfactant }}=10 \mathrm{wt} . \%$, the pore cross-sections remain monodisperse after polymerization. Though, polymerization tremendously increases $d_{\text {pore }}$ compared to $d_{\text {droplet. }}$ The reason for this increase is not known. Possible explanations are droplet coalescence or Ostwald ripening.

\section{Shape of Pore Cross-Sections: Shape Factor $S$}

As before in Section 3.1.1 and Section 3.1.2, the shape factor $S$ was calculated for the samples shown in Figure 3.16. Since the shape of the 2D pore cross-sections is always hexagonal, it could be expected that $S$ would lie between 1.05 (straight edges) and 1.10 (distorted edges). Figure 3.20 (right) depicts $S$ as function of $\beta_{\text {surfactant }}$ and Figure 3.20 (left) again shows $S$ for a distorted hexagon, for a hexagon, and a circle. Figure 3.20 (right) shows $S$ is indeed between 1.05 and 1.10. The discussion of these results will follow in Section 3.2.2. 


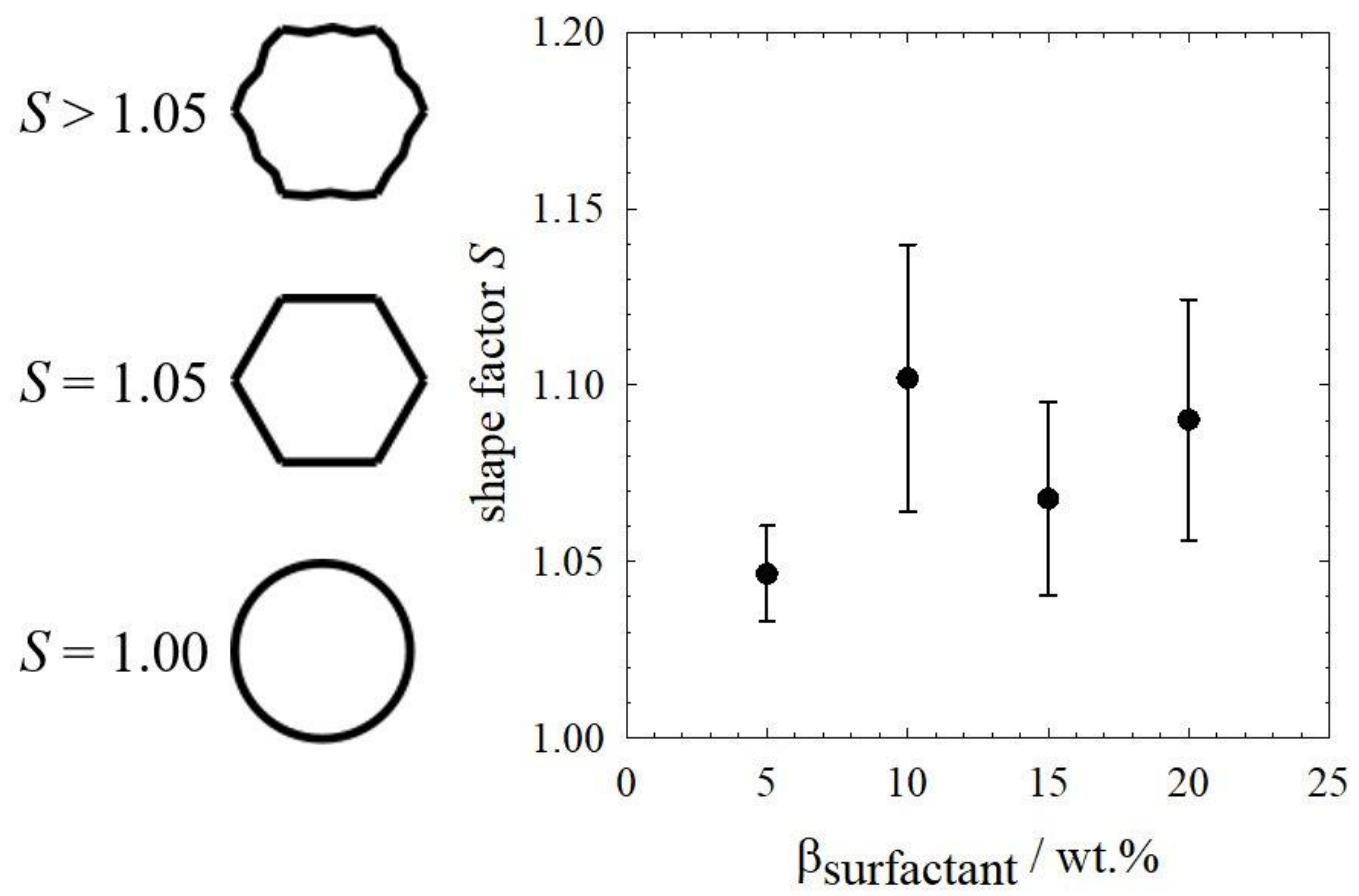

Figure 3.20: (left) Exemplary values of the shape factor $S$ for a sphere (bottom), a hexagon (middle), and a distorted hexagon (top). (right) $S$ as function of $\beta_{\text {surfactant }}$ for macroporous PS/poly-DVB at $\beta_{\text {styrene }}=50 \mathrm{wt} . \%$ and $\beta_{\mathrm{KPS}}=2.98 \mathrm{wt} . \%$.

\section{Area of Pore Wall and Layers}

In Section 3.1, the thickness of the pore wall ( $\left.t_{\text {pore wall }}\right)$ and of the layers ( $t_{\text {outer layer }}$ and $t_{\text {inner layer }}$ ) was determined. In Section 3.2, however, the area of the pore wall $A_{\text {pore wall, }}$ the area of the outer

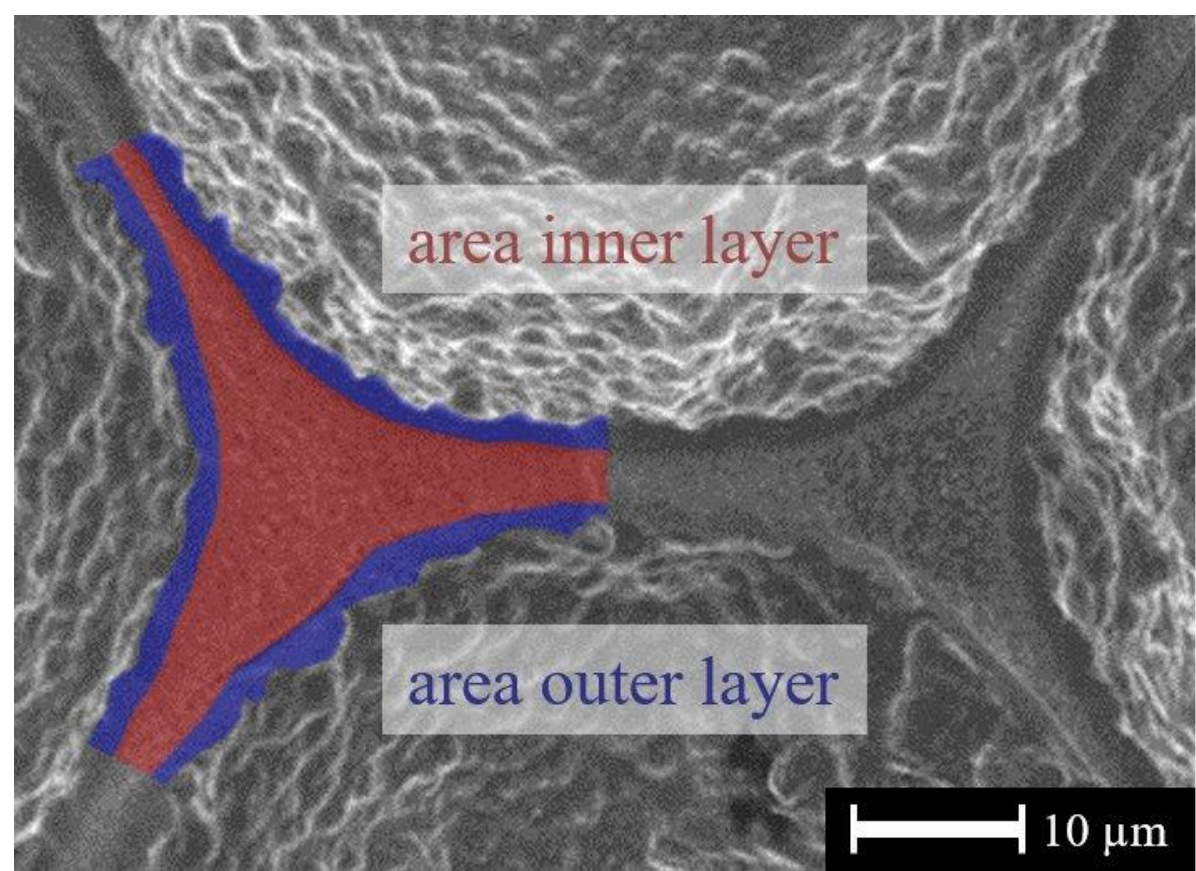

Figure 3.21: Exemplary determination of the area of the outer layer (blue) and of the inner layer (red) for one strut. 
layer $A_{\text {outer layer, }}$ and the area of the inner layer $A_{\text {inner layer }}$ were determined instead. Measuring the area turned out to be a bit more accurate than measuring the thicknesses. Figure 3.21 shows an

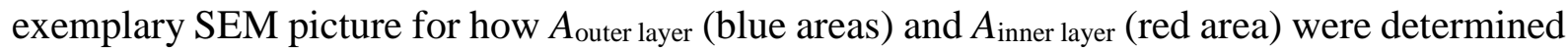
in one strut. $A_{\text {pore wall }}$ is obtained by adding $A_{\text {outer layer, and }} A_{\text {inner layer. }}$ To determine average values
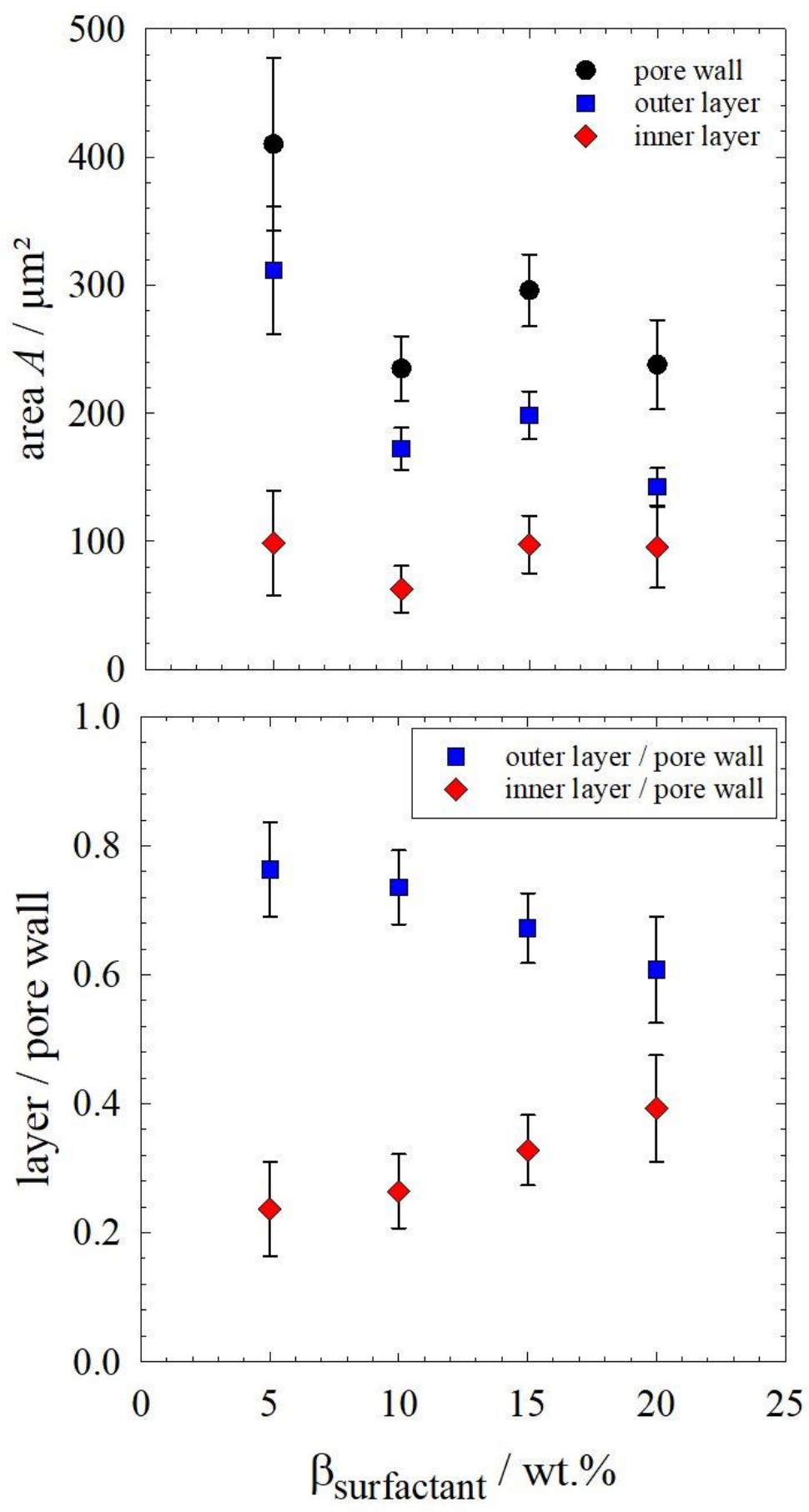

Figure 3.22: (top) Average area of the whole pore wall $A_{\text {pore wall }}$ (black circles), the outer layers $A_{\text {outer layer }}$ (blue squares), and the inner layer $A_{\text {inner layer (red diamonds) as function of } \beta_{\text {surfactant }} \text { for }}$ macroporous PS/poly-DVB at $\beta_{\text {styrene }}=50 \mathrm{wt} . \%$ and $\beta_{\mathrm{KPS}}=2.98 \mathrm{wt} . \%$. (bottom) Ratio $A_{\text {outer layer }}$

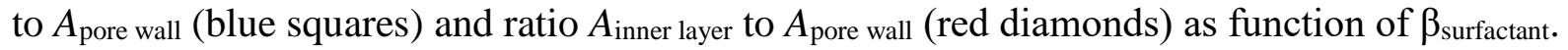


of $A_{\text {pore wall }}, A_{\text {outer layer, }}$ and $A_{\text {inner layer, }}$, several struts are examined for each sample. In Figure 3.22

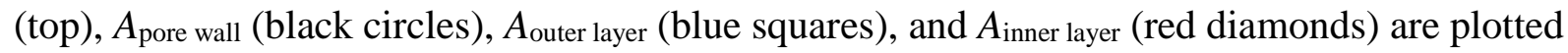

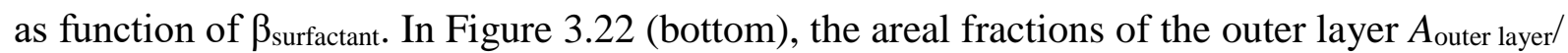
$A_{\text {pore wall }}$ and of the inner layer $A_{\text {inner layer }} / A_{\text {pore wall }}$ are plotted as function of $\beta_{\text {surfactant. Figure } 3.22}$

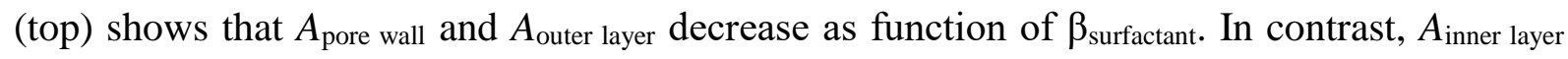
remains more or less constant when $\beta_{\text {surfactant }}$ is varied. A slight exception to these trends is found for $\beta_{\text {surfactant }}=10 \mathrm{wt} . \%$. Here, $A_{\text {pore wall }}, A_{\text {outer layer, }}$, and $A_{\text {inner layer }}$ are smaller than what the overall trend would suggest. Nevertheless, Figure 3.22 (bottom) shows a clear trend: The fraction of the outer layer decreases as function of $\beta_{\text {surfactant, }}$ while the fraction of the inner layer increases. Again, the detailed discussion of the results will follow in Section 3.2.2.

\section{Texture of Outer Layer and Inner Layer}

So far, it was not possible to investigate the texture of the outer and the inner layers. With a 100-fold, a 500-fold, and a 1000-fold magnification, the two layers could only be distinguished because of their different colors. While the outer layer appears dark grey, the inner layer appears in light grey (see Figure 3.1, Figure 3.9, and Figure 3.16). However, the texture of the outer and the inner layers becomes visible at higher magnifications. In Figure 3.23, the magnifications are 2500-fold (left column), 5000-fold (middle column), and 10000-fold (right column). The samples that were used are the KPS samples of Table 3.3 and their SEM pictures were already shown with lower magnifications in Figure 3.16. Note that for the middle and right column in Figure 3.23, another SEM had to be used in order to achieve the desired magnifications. The specifications of both SEMs can be found in the Experimental Section. Looking at the middle and right column of Figure 3.23, one sees that the outer layer appears non-porous, while the inner layer appears porous. A porous inner layer implies that material is removed at some point, while this does not happen in the outer layers. The texture of the outer layer does not change when $\beta_{\text {surfactant }}$ is varied. The texture of the inner layer also does not look different for $\beta_{\text {surfactant }}$ $=10 \mathrm{wt} . \%,=15 \mathrm{wt} . \%$, and $=20 \mathrm{wt} . \%$, while it appears less porous at $\beta_{\text {surfactant }}=5 \mathrm{wt} . \%$. This observation suggests that the porosity of the inner layer is related to or even caused by the surfactant which is why the behavior of the surfactant during the polymerization was further investigated. 

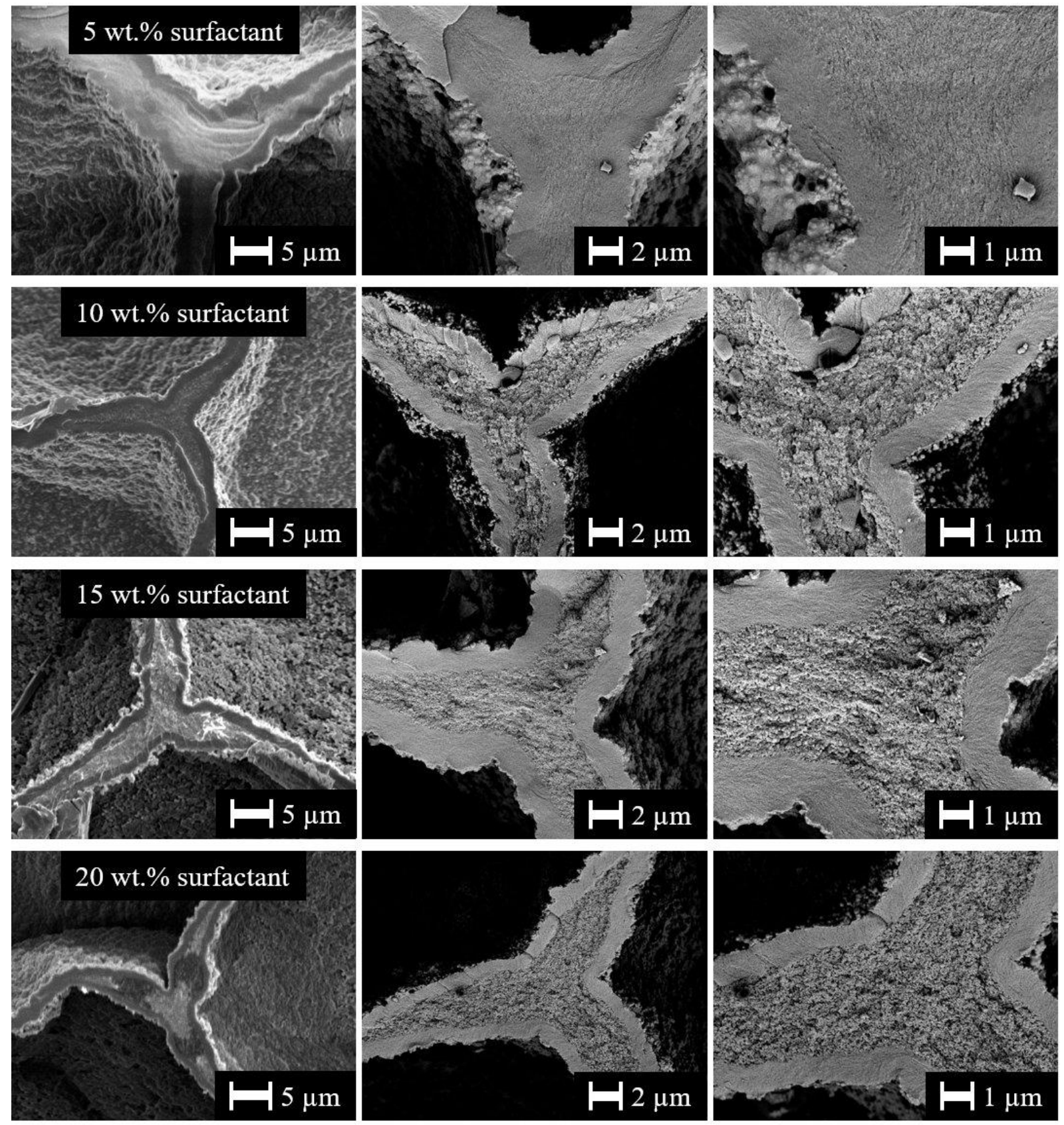

Figure 3.23: SEM pictures of macroporous PS/poly-DVB obtained via polymerization of monodisperse water-in-styrene/DVB HIPEs with $\beta_{\text {styrene }}=50$ wt. $\%, \beta_{\mathrm{KPS}}=2.98 \mathrm{wt} . \%$, and $\beta_{\text {surfactant }}=5 \mathrm{wt} . \%,=10 \mathrm{wt} . \%,=15 \mathrm{wt} . \%$, and $=20 \mathrm{wt} . \%$. The samples are the same as in Figure 3.16. The magnifications are 2500-fold (left column), 5000-fold (middle column), and 10000fold (right column).

\section{Behavior of Surfactant during Polymerization}

Assuming that the porosity of the inner layer is indeed caused by the surfactant, the question arises: why is only the inner layer porous, while the outer layer is non-porous/compact? Even for the lowest $\beta_{\text {surfactant }}$ of $5 \mathrm{wt}$.\%, the amount of surfactant is much higher than what is needed to stabilize the water/monomer interface in the HIPEs. Thus, the remaining amount of surfactant 
is simply dissolved in the continuous phase since the surfactant is insoluble in $\mathrm{H}_{2} \mathrm{O}$. Using geometrical considerations, $\beta_{\text {surfactant at interface }}$ as function of $d_{\text {droplet }}$ and of the head group area $A_{\text {head group }}$ can be calculated (see Appendix Section 6.1 (e) and 6.2 (d)). $A_{\text {head group }}$ describes the area one surfactant molecule covers at the water/monomer interface. Figure 6.5 in the Appendix shows $\beta_{\text {surfactant at interface }}$ as function of $A_{\text {head group }}$ for three different droplet diameters, namely $d_{\text {droplet }}=60 \mu \mathrm{m}$ (dashed line),$d_{\text {droplet }}=80 \mu \mathrm{m}$ (continuous line), and $d_{\text {droplet }}=100 \mu \mathrm{m}$ (dotted line). As $A_{\text {head group increases, }} \beta_{\text {surfactant at interface }}$ decreases. $A_{\text {head group }}$ can be assumed to lie between $50 \AA^{2}$ and $250 \AA^{2}$ [Wet01, Dom07] which leads to values of $\beta_{\text {surfactant at interface between } 0.06 \text { wt.\% }}$ and 0.01 wt.\%. Therefore, only a small fraction of the surfactant is located at the water/monomer interface, while the majority of the surfactant is dissolved in the continuous phase. $^{7}$

Since the majority of the surfactant molecules are simply dissolved in the continuous phase, the next question is what happens to them during polymerization. To simulate this process, a model ternary phase diagram was measured. The three components were the monomer styrene, the

\section{polystyrene}

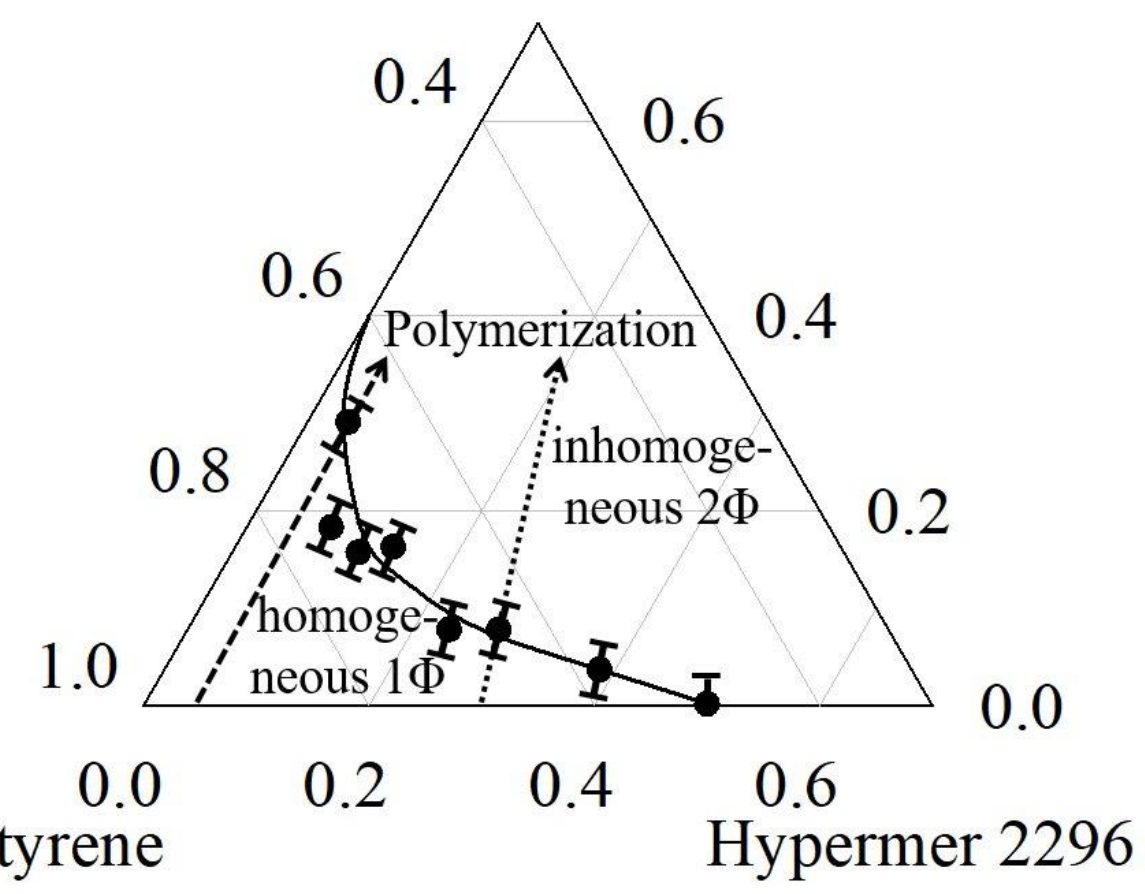

Figure 3.24: Model ternary phase diagram of the monomer styrene, the surfactant Hypermer 2296 , and the polymer polystyrene at $70{ }^{\circ} \mathrm{C}$. The black dots mark the positions of the measured phase boundaries between the homogeneous one-phase $(1 \Phi)$ and inhomogeneous two-phase $(2 \Phi)$ region. The arrows indicate the direction of the simulated polymerization.

\footnotetext{
${ }^{7} \beta_{\text {surfactant }}$ was not decreased to values below $5 \mathrm{wt} . \%$ because a too low $\beta_{\text {surfactant }}$ tampers with the generation of monodisperse droplets via microfluidics. The reason for this not understood yet.
} 
(technical, non-ionic) surfactant Hypermer 2296, and the polymer polystyrene (PS). The model ternary phase diagram was recorded at $70{ }^{\circ} \mathrm{C}$, the temperature of polymerization. Surfactant/styrene mixtures with varying compositions simulated different $\beta_{\text {surfactant. By }}$ dissolving purchasable PS in these mixtures, the polymerization was simulated which was much more practical than actually polymerizing the mixtures, stopping halfway, and then determining how far the polymerization had progressed. DVB was not included because no purchasable polyDVB could be found. $\mathrm{H}_{2} \mathrm{O}$ was also not included because the focus lay on the behavior of the surfactant during polymerization and not on its emulsification properties. PS was dissolved in the surfactant/styrene mixtures until phase separation occurred. At this point, the original surfactant/styrene mixture was added again to get back into the homogeneous one-phase region which allowed a more accurate determination of the phase boundary. Figure 3.24 shows the results of these experiments. The black dots represent the phase boundary and the two arrows indicate the direction of the simulated polymerization. In Figure 3.24, the relation between the position of the phase boundary and $\beta_{\text {surfactant }}$ is clearly seen. When $\beta_{\text {surfactant }}$ is low, high amounts of PS can be dissolved in the surfactant/styrene mixture before phase separation occurs (see Figure 3.24 (dashed arrow)). In contrast, when $\beta_{\text {surfactant }}$ is high, only low amounts of PS can be added before phase separation occurs (see Figure 3.24 (dotted arrow)). This implies that the surfactant and PS are immiscible, i.e. the polymerization leads to an increasingly bad solvent for the surfactant. The consequences of this observation will be discussed in Section 3.2.2.

\section{Formation of Water Droplets}

There was one last thing to be clarified, namely whether the porosity of the inner layer stems only from the surfactant or from a combination of surfactant and $\mathrm{H}_{2} \mathrm{O}$. In the latter case, the surfactant would have to extract $\mathrm{H}_{2} \mathrm{O}$ from the dispersed phase and form small structures - most likely droplets - in the continuous monomer phase. During polymerization, these droplets would then leave the regions close to the water/monomer interface and accumulate in the continuous phase. Since no additional energy is put into the system, the droplets would have to form spontaneously. For the KPS samples used in this Section (see Table 3.3), the interfacial tension $\gamma$ between the dispersed phase and the continuous phase was measured with Spinning Drop Tensiometry. This was done to assess if spontaneous droplet formation is possible which is typically only the case for microemulsions for which $\gamma$ can be as low as $10^{-3}-10^{-5} \mathrm{mN} \mathrm{m}^{-1}$. However, such low values are only reached at the phase inversion temperature, while $\gamma$ can increase up to $10^{-1}-1 \mathrm{mN} \mathrm{m}^{-1}$ at other temperatures [Stu09]. For the four samples studied in 
this Section, $\gamma$ was between $0.5 \mathrm{mN} \mathrm{m}^{-1}$ and $1.0 \mathrm{mN} \mathrm{m}^{-1}$ for temperatures between $25-70{ }^{\circ} \mathrm{C}$. It was found that $\gamma$ decreases with increasing $\beta_{\text {surfactant }}$ and increases with increasing temperature. Since spontaneous droplet formation is thus theoretically possible, this hypothesis was put to the test with a simple visual experiment. If spontaneous droplet formation actually occurs, a monomer phase that is brought into contact with $\mathrm{H}_{2} \mathrm{O}$ will eventually become turbid if the droplets become big enough to scatter light. At first, the two phases were prepared separately. The monomer phase consisted of styrene, DVB, and surfactant with $\beta_{\text {styrene }}$ being kept constant

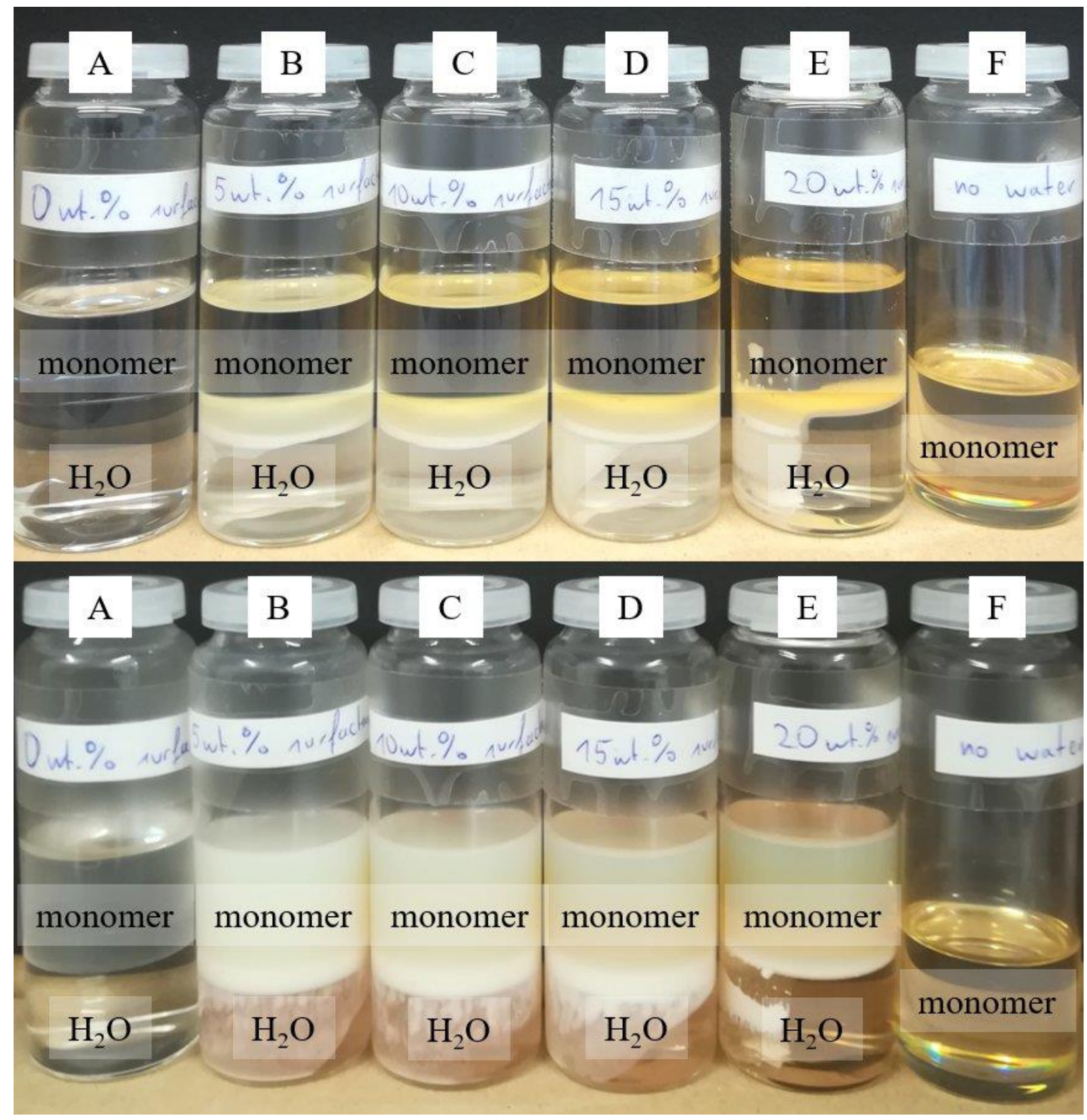

Figure 3.25: Visual development of styrene/DVB mixtures with surfactant mass fractions of 0 wt.\% (A), 5 wt.\% (B), 10 wt.\% (C), 15 wt.\% (D), and 20 wt.\% (E) exposed to $\mathrm{H}_{2} \mathrm{O}$. When both surfactant molecules and $\mathrm{H}_{2} \mathrm{O}$ are present (B-E), the monomer phases become turbid. When either surfactant (A) or $\mathrm{H}_{2} \mathrm{O}(\mathrm{F})$ is missing, the monomer phases remain clear. The surfactant mass fraction in the sample without $\mathrm{H}_{2} \mathrm{O}(\mathrm{F})$ was 10 wt.\%. 
at $50 \mathrm{wt} . \%$ and $\beta_{\text {surfactant }}$ being varied between $0-20 \mathrm{wt} . \%$. Next, the monomer phase was carefully put over $\mathrm{H}_{2} \mathrm{O}$ to limit mixing of the two phases which would result in unwanted emulsification. Figure 3.25 (top A - E) depicts the appearance of the samples directly after the two phases were put together. Figure 3.25 (top F) shows a monomer phase with $\beta_{\text {surfactant }}=10$ wt.\% where no $\mathrm{H}_{2} \mathrm{O}$ was added. This sample was prepared to serve as a comparison to the other five samples. Figure 3.25 (bottom) depicts the same samples as in the top row after 24 h. Figure 3.25 (F) shows than when no $\mathrm{H}_{2} \mathrm{O}$ is made available to the monomer phase, it remains clear after $24 \mathrm{~h}$. Note that this is also the case for $\beta_{\text {surfactant }}=5 \mathrm{wt} . \%, 15 \mathrm{wt} . \%$, and $20 \mathrm{wt} . \%$. The monomer phase also remains clear when $\beta_{\text {surfactant }}=0$ wt.\% (see Figure $3.25(\mathrm{~A})$ ). In contrast, the monomer phase becomes turbid and later even white when $\beta_{\text {surfactant }}$ is between $5-20 \mathrm{wt}$.\% (see Figure $3.25(B-E)$ ). Thus, both surfactant and $\mathrm{H}_{2} \mathrm{O}$ need to be present for the turbidity to develop, i.e. for droplets to form. It was observed that the turbidity and the whiteness of the

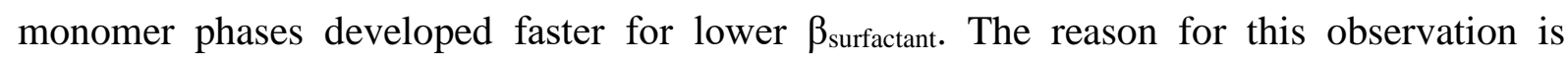
discussed in Section 3.2.2. ${ }^{8}$

To better understand the droplet formation, small angle neutron scattering (SANS) experiments were performed. For this purpose, the two monomers styrene and DVB were replaced by deuterated styrene and $\mathrm{H}_{2} \mathrm{O}$ was replaced by $\mathrm{D}_{2} \mathrm{O}$. After preparing a sample with $\beta_{\text {surfactant }}=10$ wt.\%, small fractions of the monomer phase were removed after 8 minutes, 13.5 hours, and 1.5 days and SANS spectra were recorded. Figure 3.26 depicts the $I$ - $q$-curve (black circles) of the sample that was removed after 8 minutes. The curve was fitted to determine the shape and size of the structures which were so far assumed to be droplets. The fit was done with a Gaussian shell model [Fos08] where $I(q)$ (blue line) is calculated by combining a form factor $P(q)$ (red line) and a structure factor $S(q)$ (light green line). Figure 3.26 (inset) depicts the intensity $I$ of the samples removed after 8 minutes (black circles), after 13.5 hours (orange squares), and after 1.5 days (dark green diamonds) as function of the scattering vector $q$. The fit of the $I(q)$-curve in Figure 3.26 is in line with vesicles that consist of surfactant bilayers and contain only a very small amount of water. The diameter of the vesicles was determined to be $11 \pm 5 \mathrm{~nm}$. However, the calculated $I(q)$-curve (blue line) describes the experimental data only for small $q$, while it deviates a bit for higher $q$. Thus, the structure derived from the fitting procedure should be handled with care. Looking at Figure 3.26 (inset), one sees that the three measured $I(q)$-curves look similar. This implies that the shape and size of the structures does not change significantly

\footnotetext{
${ }^{8}$ The length scale and volume of the samples shown in Figure $3.25\left(\mathrm{~cm}\right.$ and $\left.\mathrm{cm}^{3}\right)$ is not comparable to that of the real samples $\left(\mu \mathrm{m}\right.$ and $\mu \mathrm{m}^{3}$ ). Thus, the time frame of Figure 3.25 it not transferable to the real samples.
} 


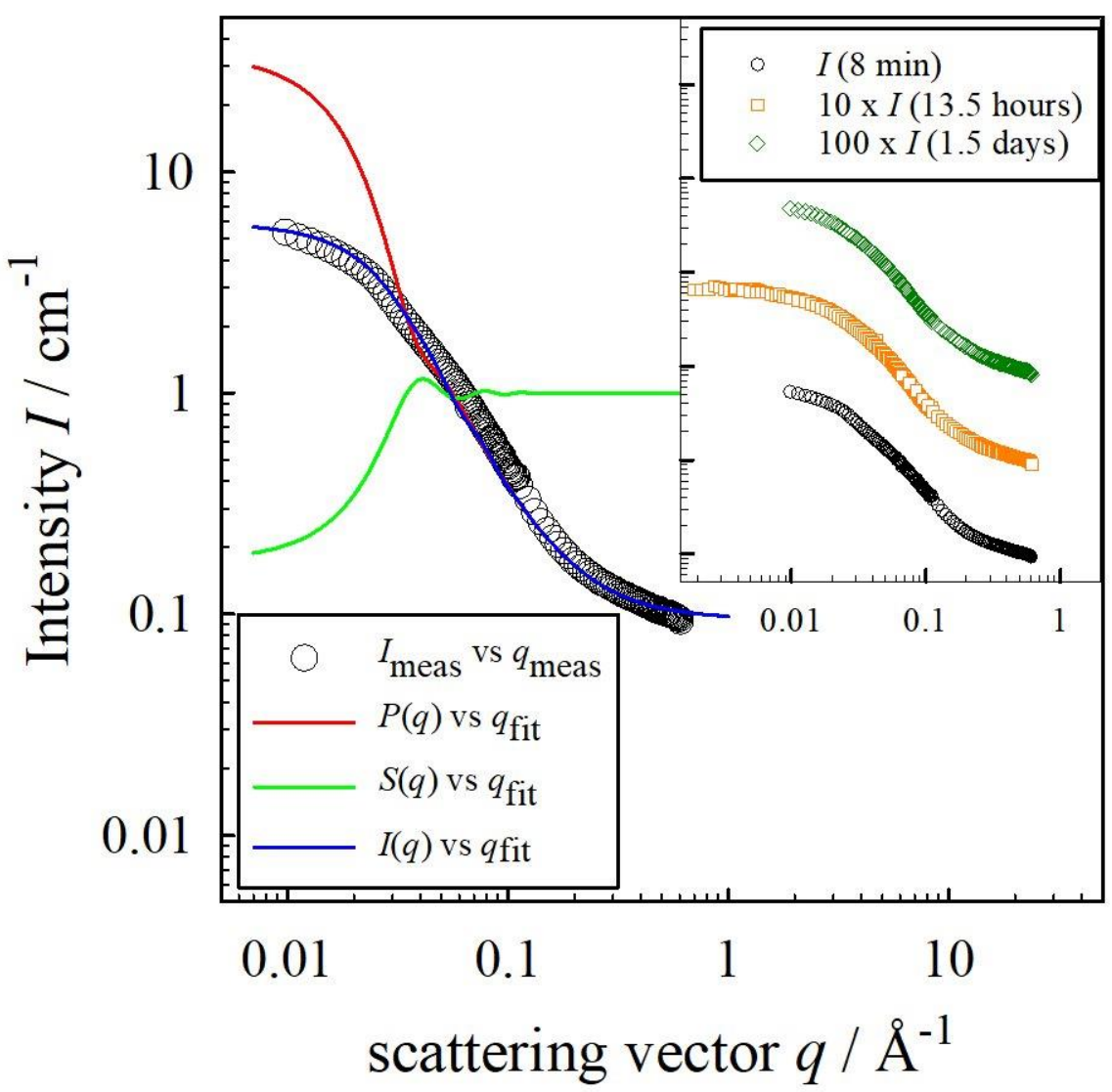

Figure 3.26: Measured intensity $I_{\text {meas }}$ after 8 minutes (black circles), form factor $P(q)$ (red line), structure factor $S(q)$ (light green line), and calculated intensity $I(q)$ (blue line) as function of the scattering vector $q$. (inset) Measured intensities $I$ after 8 minutes (black circles), after 13.5 hours (orange squares), and after 1.5 days (dark green diamonds) as function of the scattering vector $q$. For the sake of clarity, the intensities of the curves measured after 13.5 hours (orange squares) and after 1.5 days (dark green diamonds) were multiplied by a factor of 10 and 100, respectively.

even after 13.5 hours or 1.5 days. Since the findings from the SANS measurements are not completely convincing, the sample was additionally investigated with freeze fracture electron microscopy (FFEM) and dynamic light scattering (DLS).

For FFEM, the sample shown in Figure 3.25 (C) was prepared again, i.e. $\beta_{\text {surfactant }}$ was set to 10 wt. $\%$ and $\beta_{\text {styrene }}=50 \mathrm{wt} . \%$. Small fractions of the monomer phase were removed, frozen, and coated with carbon. The exact process is described in the Experimental Section. The resulting samples were investigated with a transmission electron microscope (TEM). Three exemplary pictures are shown in Figure 3.27. When looking at the FFEM pictures in Figure 3.27, one sees two structures with different size ranges. Both structures appear circular in the 2D FFEM pictures and are therefore spherical in 3D. Though the vesicles that were suggested from fitting the SANS data are spherical as well, their imprint in FFEM would have another, ring like appearance. The imprints found in Figure 3.27 are more likely caused by droplets. The smaller 

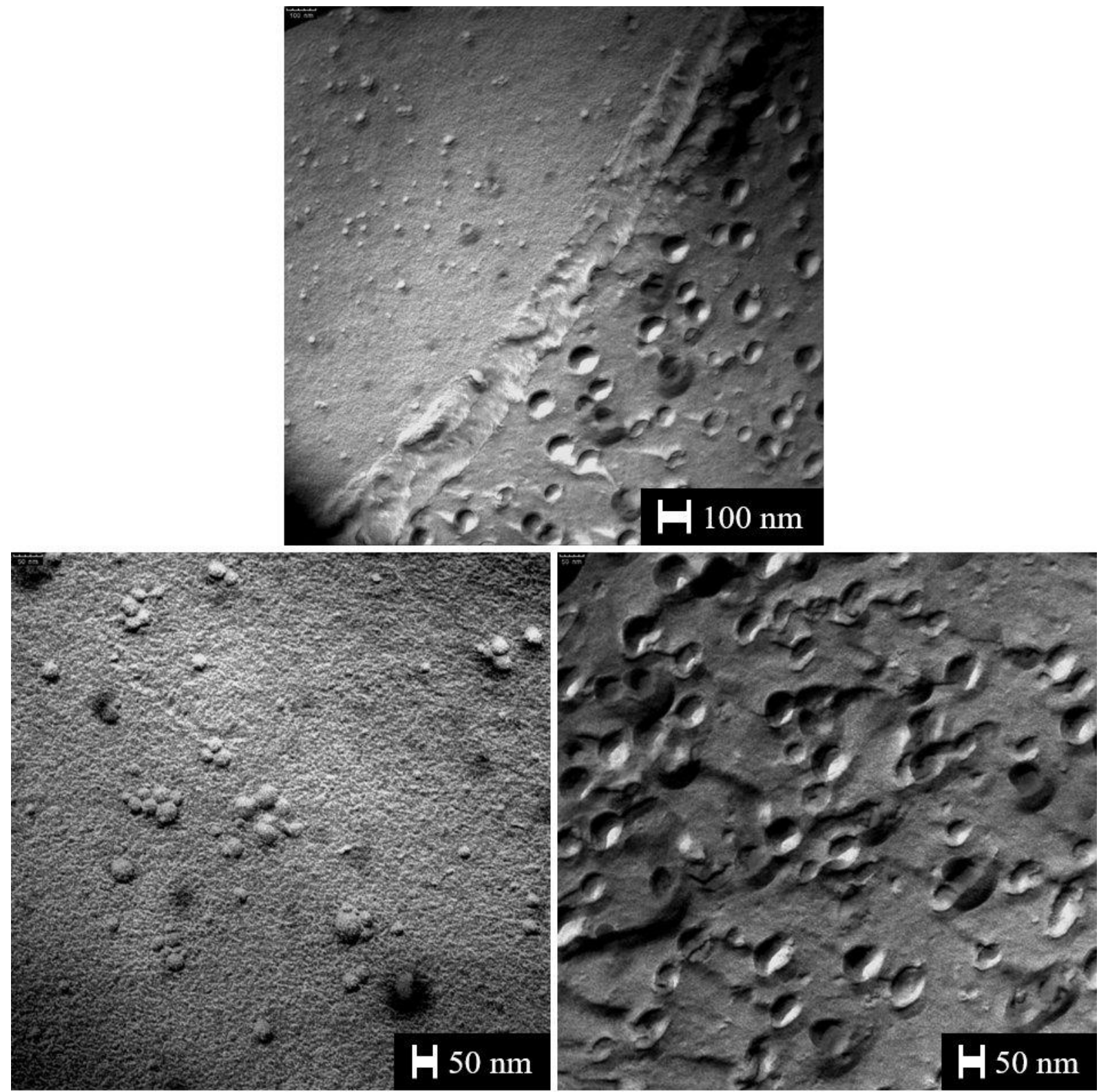

Figure 3.27: FFEM pictures of a 50/50 styrene/DVB mixture with a surfactant mass fraction of $10 \mathrm{wt} . \%$ that was placed over $\mathrm{H}_{2} \mathrm{O}$.

structures are $\sim 25 \mathrm{~nm}$ in diameter and thus roughly match the size already determined by SANS. The larger structures have a diameter of $\sim 100 \mathrm{~nm}$. The top of Figure 3.27 shows that there is no gradual change between the two sizes, but an abrupt one. The reason for this is not known.

Finally, the sample containing $\beta_{\text {surfactant }}=10 \mathrm{wt} . \%$ at $\beta_{\text {styrene }}=50 \mathrm{wt} . \%$ was prepared again. Immediately after preparing the sample, the monomer phase was placed into the beam path of a DLS device and scattered light was recorded at a fixed angle of $90^{\circ}$. With the help of a correlation function, the hydrodynamic radius $r_{\mathrm{Hyd}}$ (white points) as well as its deviation (black bars) were calculated by the software Z.1 from LS Instruments. The evolution of $r_{\text {Hyd }}$ was deter- 


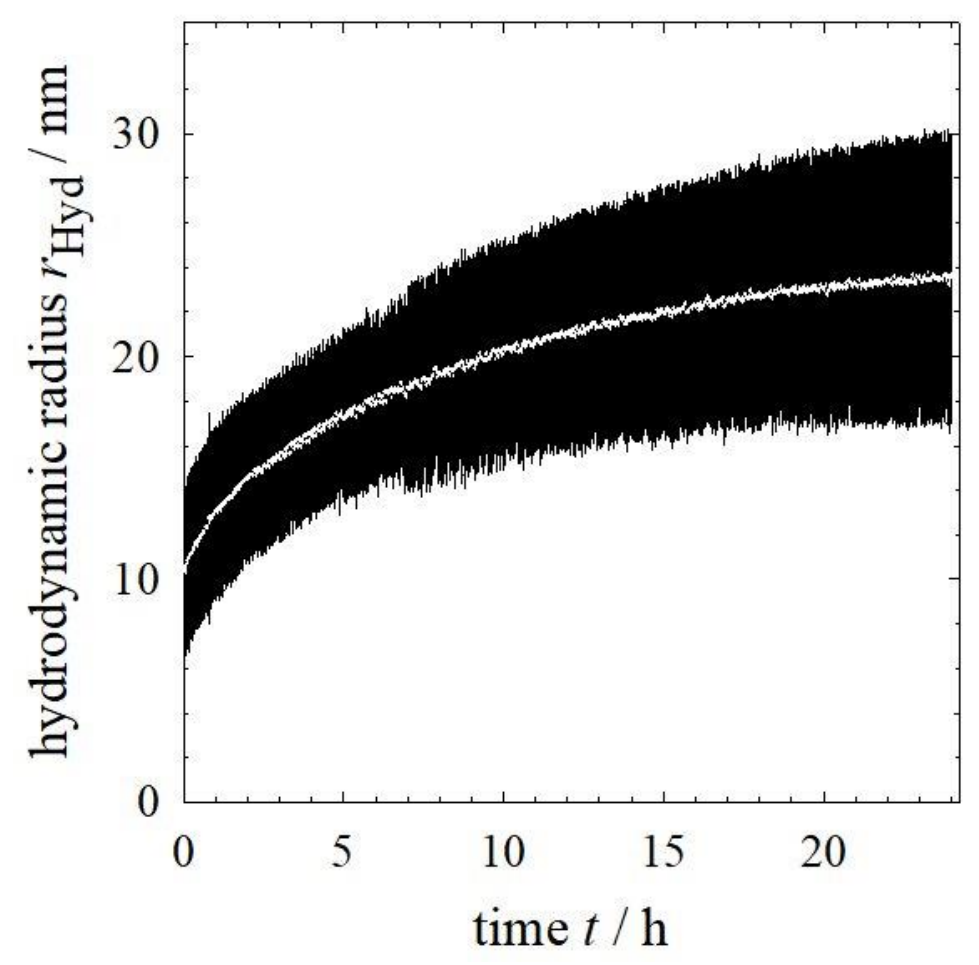

Figure 3.28: DLS curve of a 50/50 styrene/DVB mixture with a surfactant mass fraction of 10 wt.\% that was put over $\mathrm{H}_{2} \mathrm{O}$. The white dots are the measured hydrodynamic radius $r_{\mathrm{Hyd}}$ and the black bars are the measured width.

mined for a duration of $24 \mathrm{~h}$ which is depicted in Figure 3.28. Figure 3.28 shows that moments after the sample was prepared, the detected structures have an average $r_{\mathrm{Hyd}}$ of $11 \mathrm{~nm}$. As time passes, $r_{\text {Hyd }}$ increases rapidly in the beginning and slower towards the end of the measurement. After $24 \mathrm{~h}, r_{\text {Hyd }}$ has increased to $\sim 24 \mathrm{~nm}$. The deviation from the average $r_{\text {Hyd }}$ also increases with time. In the beginning, the deviation is $\sim 4 \mathrm{~nm}$ and increases up to $\sim 7 \mathrm{~nm}$ after $24 \mathrm{~h}$ have passed. Note that the results strongly depend on the distance between the water/monomer interface and the height at which the laser beam crosses the monomer phase. When this distance is larger, the increase of $r_{\mathrm{Hyd}}$ in Figure 3.28 requires more time to occur. These results as well as of all other results of Section 3.2.1 will be discussed in Section 3.2.2. 


\subsubsection{Discussion of Experimental Results}

The results of Section 3.1 disproved the idea of osmotic transport of DVB as the reason for the transformation from spherical droplets to polyhedral pores when KPS is used as initiator. Nevertheless, two main questions remained unanswered. The first of these is (1) why the shape of the pore cross-sections is hexagonal after the polymerization. This requires the area of the water/monomer interface to increase during the polymerization and thus, a certain mechanism counteracts capillary pressure. The second question is (2) why the pore walls consist of two quite similar looking non-porous outer layers and one porous inner layer. To answer these questions, the experiments in Section 3.2.1 were conducted. The surfactant mass fraction $\beta_{\text {surfactant }}$ was varied to investigate its influence on the morphology of the macroporous PS/polyDVB samples. More precisely, the shape of the pore cross-sections and the development of the layer areas was examined with SEM. Regardless of which $\beta_{\text {surfactant }}$ is used, the pore crosssections are always hexagonal when KPS is used as initiator. Therefore, the mechanism that increases the area of the water/monomer interface occurs regardless of how large $\beta_{\text {surfactant }}$ is. However, the area of the pore wall and its layers depend on $\beta_{\text {surfactant. While }} A_{\text {pore wall }}$ and $A_{\text {outer }}$

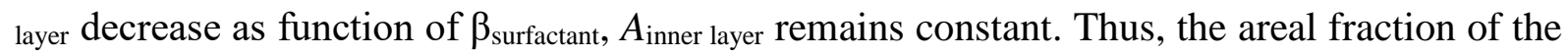

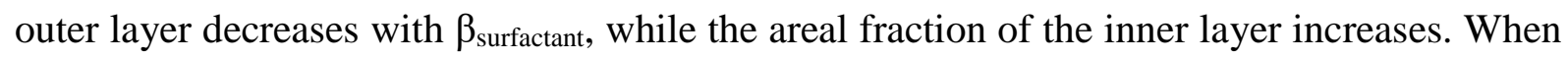
AIBN is used as initiator, the pore cross-sections are always circular and the pore walls are porous. To determine the effect of the polymerization on the solubility of the surfactant molecules in the continuous phase, a ternary phase diagram of surfactant, styrene, and polystyrene (PS) was measured. It was found that the surfactant is insoluble in PS, which implies that the polymerization drives the surfactant out of the continuous phase. By and large, the results and their interpretations allow the construction of a mechanism that illustrates what is happening during polymerization (see Figure 3.29). The surfactant molecules consist of a hydrophilic head (dark blue) and a hydrophobic tail (red). The water droplets of the dispersed phase are depicted in light blue and the continuous phase is shown in orange. The polymerization is illustrated by the gradual change in color from orange (unpolymerized) via light brown (partially polymerized) to black (fully polymerized). Note that the sizes of the surfactant molecules, of the water droplets, and of the space between two droplets are not to scale. 


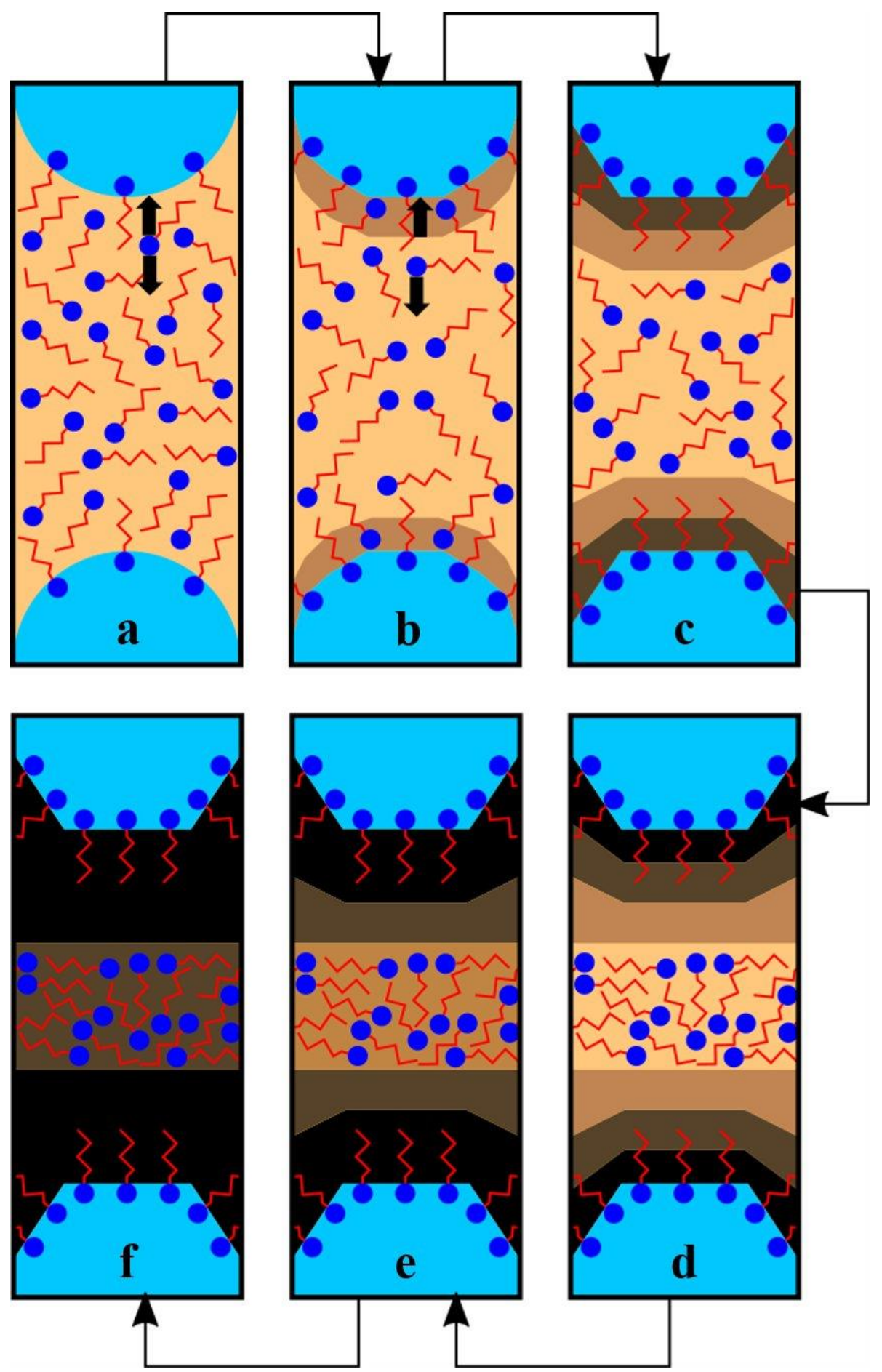

Figure 3.29: Schematic drawing of the mechanism that increases the area of the water/monomer interface and that creates non-porous outer layers and a porous inner layer in the pore wall.

Before polymerization starts, the shape of the droplets is spherical (circular in 2D scheme of Figure 3.29) and the surfactant either stabilizes the water/monomer interface or is evenly distributed in the continuous phase (see Figure 3.29 (a)). Since the polymerization starts at the interface with the water-soluble initiator KPS, a small layer of partially polymerized continuous phase forms close to the water/monomer interface (see Figure 3.29 (b), light brown layer). The 
surfactant is now less soluble here compared to other regions where no polymerization has taken place so far. Thus, the polymerization close to the water/monomer interface prompts the surfactant molecules in this region to diffuse away. Here, two options exist, namely diffusion (1) to the water/monomer interface or (2) to the interior of the monomer phase.

(1) The water/monomer interface is very close to the polymerization zone and diffusion towards it is possible at the beginning since the continuous phase is only partially polymerized (see Figure 3.29 (b) arrow upwards). At the interface, surfactant molecules create an oversaturation which, in turn, increases the interfacial area [Jia99, Pat12, Giu17]. Note that the surfactant will remain at the interface because it is insoluble in $\mathrm{H}_{2} \mathrm{O}$. By increasing its area, the shape of the interface changes from spherical to polyhedral. In Figure 3.29, this is illustrated by the change of the droplet cross-sections from circular (see Figure 3.29 (a)) via an intermediary state (see Figure 3.29 (b)) to hexagonal (see Figure 3.29 (c)). Note that the ongoing polymerization prevents further surfactant diffusion towards the water/monomer interface since the continuous phase becomes too polymerized for diffusion to happen (see Figure 3.29 (c), brown layers).

(2) The second option for the surfactant molecules is to diffuse in the opposite direction, i.e. to the unpolymerized continuous phase (see Figure 3.29 (b) arrow downwards). In contrast to diffusion towards the interface, this process continues with the ongoing polymerization. In the course of the polymerization, the region close to the water/monomer interface becomes more polymerized (see Figure 3.29 (b), light brown layers $\rightarrow$ (c), dark brown layers $\rightarrow$ (d), black layers)) and the polymerization proceeds further into the continuous phase (see Figure 3.29 (b), light brown layers $\rightarrow(\mathrm{c})$, light brown layers $\rightarrow(\mathrm{d})$, light brown layers)). Thus, two surfactantfree layers adjacent to the water droplets of the dispersed phase and one surfactant-rich inner layer in the interior of the continuous phase form (see Figure 3.29 (d)). Once the surfactant molecules are trapped inside the continuous phase, they remain there until eventually polymerization catches up (see Figure $3.29(\mathrm{~d} \rightarrow \mathrm{e} \rightarrow \mathrm{f})$ ). When the samples are later purified by Soxhlet extraction, the surfactant is washed out. This causes the porosity of the inner layer, while the outer layer remains non-porous. When $\beta_{\text {surfactant increases, more surfactant molecules }}$ can accumulate in the interior of the continuous phase. Thus, the thickness of the inner layer increases with increasing $\beta_{\text {surfactant }}$ as observed in the experiments in Section 3.2.1. Note that the overall size of the pore wall decreases as a function of $\beta_{\text {surfactant }}$ because less polymerizable material is available at higher $\beta_{\text {surfactant }}$.

In conclusion, surfactant diffusion towards the water/monomer interface is responsible for the polyhedral shape of the pores, while surfactant diffusion towards the interior of the continuous 
phase is responsible for the layered structure of the pore walls. Complementary experiments were conducted to examine whether the surfactant is solely responsible for the porosity of the inner layer. It was found that small droplets form in the continuous phase which grow in size over time. Initially, the radius of the droplets is $\sim 10 \mathrm{~nm}$ and they consist only of surfactant. Over time, the droplets grow in size by taking up water. Thus, the porosity of the inner layer is not solely caused by the surfactant, but by a combination of surfactant and water. The small water droplets are not shown in Figure 3.29 for the sake of clarity. 


\subsection{Mechanical Behavior}

The mechanical properties of macroporous PS/polyDVB obtained from polymerizing monodisperse water-in-styrene/DVB HIPEs are dealt with in Section 3.3. More precisely, the focus is put on how (a) the type of initiator, (b) the styrene/DVB mass ratio ( $\left.\beta_{\text {styrene }}\right)$, (c) the amount of initiator $\left(\beta_{\text {initiator }}\right)$, and (d) the amount of surfactant $\left(\beta_{\text {surfactant }}\right)$ influence the yield

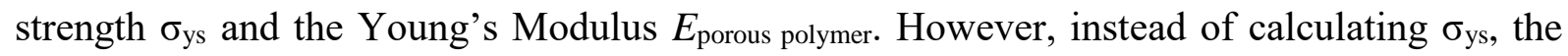
stress at which the linear elastic region goes over into the Plateau region $F_{\max }$ was determined. Emphasis is also put on the question whether monodisperse macroporous PS/polyDVB has enhanced mechanical properties compared to polydisperse macroporous PS/polyDVB. The morphology was determined from investigating one sample with SEM, while the stress-straincurves were recorded from four other samples of the same composition.

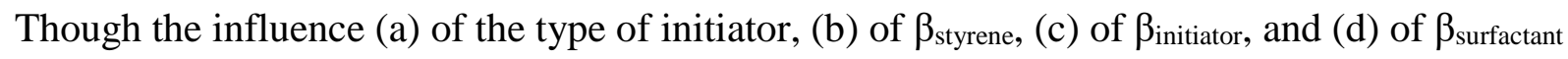
were all investigated, only (b) the variation of $\beta_{\text {styrene }}$ resulted in a meaningful trend. This is why

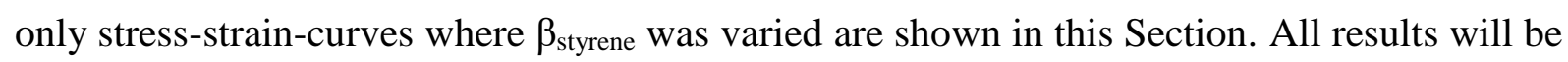
summed up in Table 3.9 at the end of this Section. The largest differences were found between $\beta_{\text {styrene }}=90 \mathrm{wt} . \%$ and $\beta_{\text {styrene }} \leq 70 \mathrm{wt} . \%$. The most striking difference were not the values of

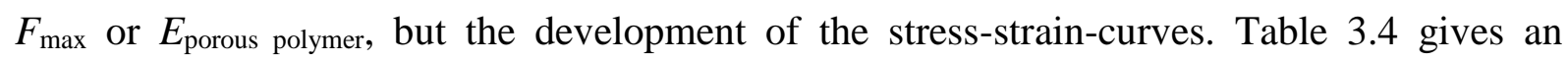
overview of the compositions whose stress-strain-curves will be shown in Figure 3.30 - Figure 3.33. $\beta_{\text {styrene }}$ was either $50 \mathrm{wt} . \%$ or $90 \mathrm{wt} . \%$ and the initiator was either KPS or AIBN. $\beta_{\text {surfactant }}$ was kept at a constant value of $10 \mathrm{wt} . \%$.

Table 3.4: Type of initiator, $\beta_{\text {styrene, }} \beta_{\text {initiator, }}$ and $\beta_{\text {surfactant }}$ of the samples whose the stress-straincurves are shown in Figure 3.30 - Figure 3.33.

\begin{tabular}{|c|c|c|c|c|}
\hline Type of Initiator & $\boldsymbol{\beta}_{\text {styrene }} / \mathbf{w t . \%}$ & $\boldsymbol{\beta}_{\text {initiator }} / \mathbf{w t . \%}$ & $\boldsymbol{\beta}_{\text {surfactant }}$ / wt.\% & Figure \\
\hline water-soluble KPS & 50 & 2.98 & 10 & Figure 3.30 \\
\hline monomer-soluble AIBN & 50 & 1.82 & 10 & Figure 3.31 \\
\hline water-soluble KPS & 90 & 2.98 & 10 & Figure 3.32 \\
\hline monomer-soluble AIBN & 90 & 1.82 & 10 & Figure 3.33 \\
\hline
\end{tabular}

Each Figure shows both the complete development of the measured stress-strain-curves (top left) and the stress-strain-curves at low strains (top right) since this is the range where $F_{\max }$ and $E_{\text {porous polymer }}$ are determined. Note that measurements of the stress-strain-curves were either stopped automatically by the software when the stress dropped to $80 \%$ of its maximum value or manually by the experimenter. In Figure 3.30 and Figure 3.31, measurements were manually stopped at some point in the plateau region once several pieces of each sample had broken off from the sample. Most often, these pieces originated from the top of the sample which was in 
contact with the load cell and broke off at the rim of the sample. In Figure 3.32 and Figure 3.33, measurements were manually stopped once the densification region of the stress-strain-curves had been reached. In addition to the stress-strain-curves, each Figure also shows the SEM pictures of a sample with the same composition. The magnifications of the SEM pictures were 100-fold (bottom left), 500-fold (bottom middle), and 1000-fold (bottom right). Figure 3.30 shows the stress-strain-curves and SEM pictures for $\beta_{\text {styrene }}=50 \mathrm{wt} . \%$ and $\beta_{\mathrm{KPS}}=2.98 \mathrm{wt} . \%$.
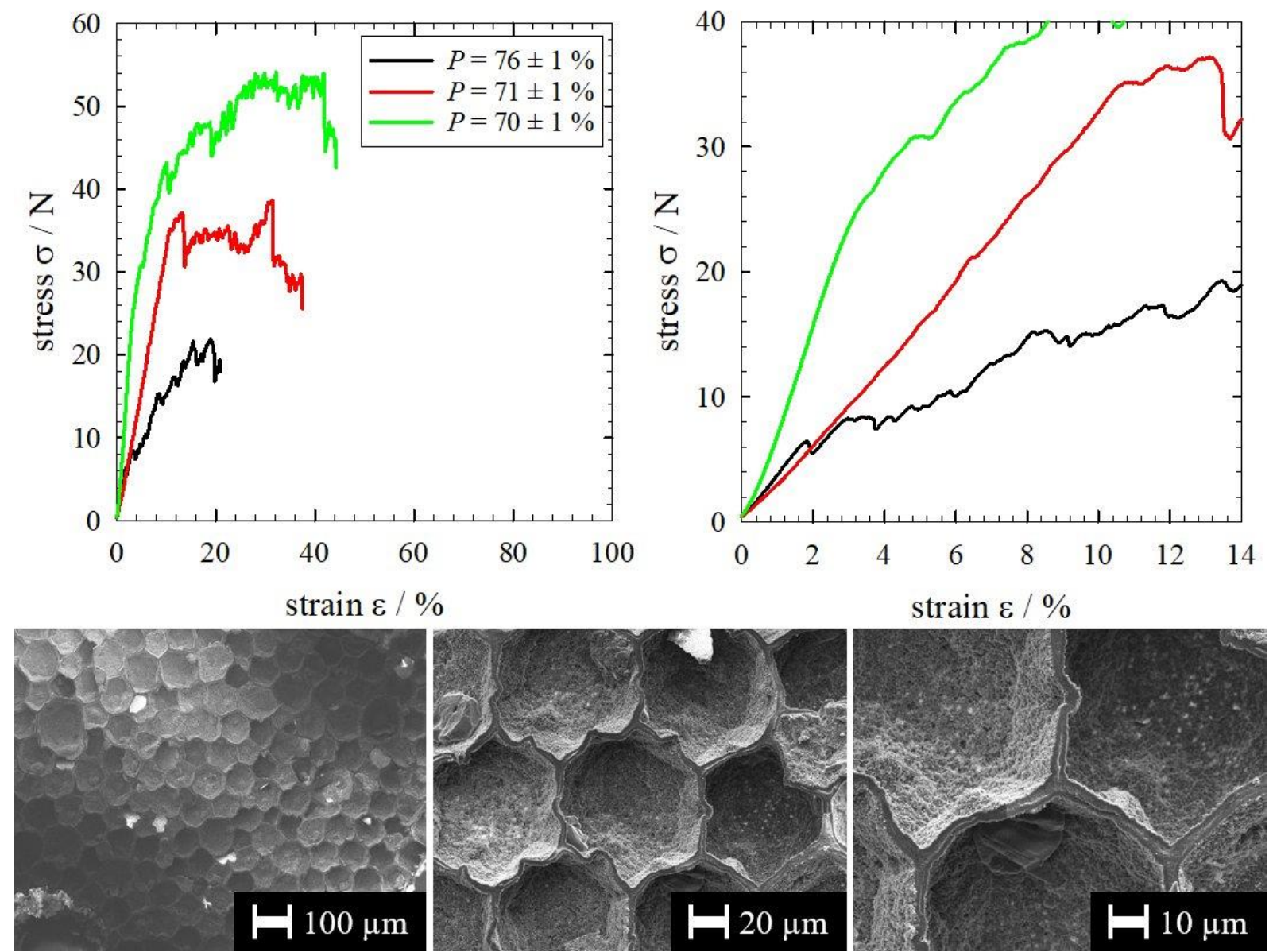

Figure 3.30: (top) Stress-strain-curves and (bottom) SEM pictures of macroporous PS/polyDVB obtained via polymerization of monodisperse water-in-styrene/DVB HIPEs with $\beta_{\text {styrene }}=$ $50 \mathrm{wt} . \%, \beta_{\mathrm{KPS}}=2.98 \mathrm{wt} . \%$, and $\beta_{\text {surfactant }}=10 \mathrm{wt} . \%$. The stress-strain-curves on the top right are magnifications of the stress-strain-curves on the top left at lower strain. The magnifications of the SEM pictures are 100-fold (bottom left), 500-fold (bottom middle), and 1000-fold (bottom right).

The SEM pictures in Figure 3.30 (bottom) show that the pores are close-packed and fairly monodisperse $\left(d_{\text {pore }}=77 \pm 4 \mu \mathrm{m}\right)^{9}$. Furthermore, the $2 \mathrm{D}$ pore cross-sections are hexagonal $(S=$ $1.10 \pm 0.03$ ) and the pore walls consist of three layers. Ideally, the porosity $P$ is $74 \%$ since this

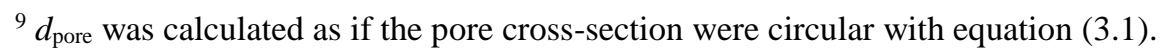


is the volume fraction of the dispersed phase in the monodisperse HIPEs. If $P<74 \%$, the pores are not close-packed in the whole sample. If $P>74 \%$, an additional porosity like the one found in the inner layer of the pore walls must be taken into account. In Section 2.3.2 it was explained that for open-pore porous polymers, $E_{\text {porous polymer }} / E_{\text {polymer }}$ ought to be directly proportional to ( $\left.\rho_{\text {porous polymer }} / \rho_{\text {polymer }}\right)^{2}$ (see equation (2.24)) and that the proportionality constant $C_{\mathrm{C}}$ ought to be $=1$. For closed-pore porous polymers, the relation between the relative Young's Modulus and $\rho_{\text {rel }}$ is more difficult (see equation (2.25)). Since the necessary parameters $\Phi$ and $v^{*}$ are not known, equation (2.24) is used also for the closed-pore polymer. Non-porous samples that were polymerized from pure styrene/DVB mixtures were used to determine $\rho_{\text {polymer }}$ and $E_{\text {polymer. The }}$ determined values for $P, E_{\text {porous polymer, }} F_{\max }$, and $C_{\mathrm{C}}$ as well as their average values are summed up in Table 3.5.

Table 3.5: Porosity $P, E_{\text {porous polymer }}, F_{\max }$, and $C_{\mathrm{C}}$ of the samples shown in Figure 3.30 where $\beta_{\text {styrene }}=50 \mathrm{wt} . \%$ and $\beta_{\mathrm{KPS}}=2.98 \mathrm{wt} . \%$.

\begin{tabular}{|c|c|c|c|c|}
\hline & Porosity $\boldsymbol{P} / \boldsymbol{\%}$ & $\boldsymbol{E}_{\text {porous polymer } / \mathbf{M P a}}$ & $\boldsymbol{F}_{\max } / \mathbf{N}$ & $\boldsymbol{C}_{\mathbf{C}}$ \\
\hline black stress-strain-curve & $76 \pm 1$ & 39 & 8 & $1.76 \pm 0.36$ \\
\hline red stress-strain-curve & $71 \pm 1$ & 27 & 36 & $0.91 \pm 0.18$ \\
\hline green stress-strain-curve & $70 \pm 1$ & 87 & 43 & $2.56 \pm 0.52$ \\
\hline average & $\mathbf{7 3} \pm \mathbf{4}$ & $\mathbf{5 1} \pm \mathbf{3 6}$ & $\mathbf{2 9} \pm \mathbf{2 1}$ & $\mathbf{1 . 9} \pm \mathbf{1 . 2}$ \\
\hline
\end{tabular}

Since the average porosity is $P=70 \pm 3 \%$, the samples were obviously not fully sedimented. The average mechanical values one obtains from the stress-strain-curves are $E_{\text {porous polymer }}=51$ $\pm 36 \mathrm{MPa}$ and $F_{\max }=29 \pm 21 \mathrm{~N}$. However, for both $E_{\text {porous polymer }}$ and $F_{\max }$, the individual values deviate largely from each other. An explanation for this cannot be given. The same is true for $C_{\mathrm{C}}$ since $C_{\mathrm{C}}=1.9 \pm 1.2$. Figure 3.30 (top) shows that the stress-strain-curves are elastic-brittle. This is also true when KPS is substituted by AIBN (see Figure 3.31). Here, $\beta_{\mathrm{AIBN}}=1.82 \mathrm{wt} . \%$ and $\beta_{\text {styrene }}$ remains at $50 \mathrm{wt} . \%$. 

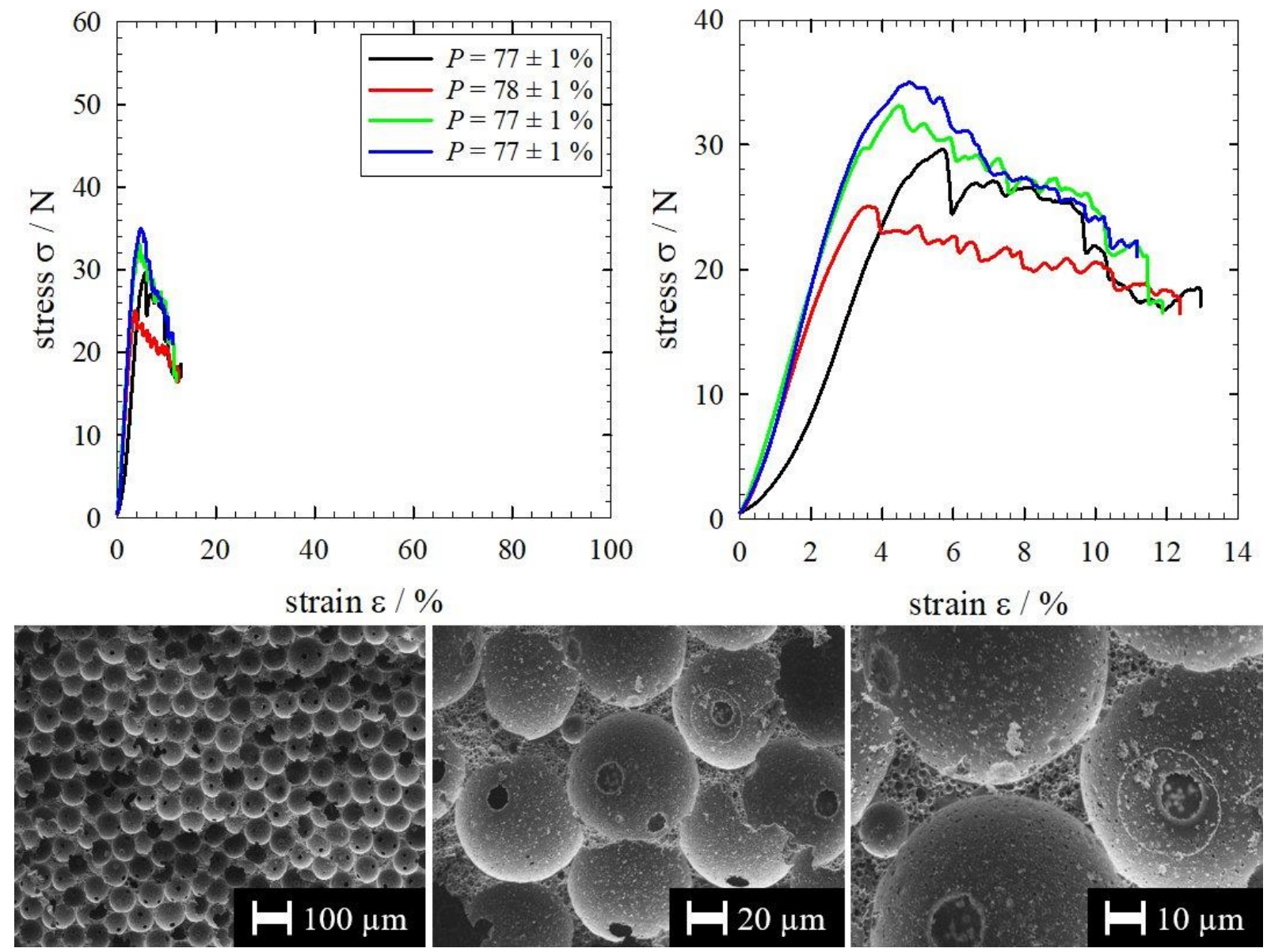

Figure 3.31: (top) Stress-strain-curves and (bottom) SEM pictures of macroporous PS/polyDVB obtained via polymerization of monodisperse water-in-styrene/DVB HIPEs with $\beta_{\text {styrene }}=$ $50 \mathrm{wt} . \%, \beta_{\text {AIBN }}=1.82 \mathrm{wt} . \%$, and $\beta_{\text {surfactant }}=10 \mathrm{wt} . \%$. The stress-strain-curves on the top right are magnifications of the stress-strain-curves on the top left at lower strain. The magnifications of the SEM pictures are 100-fold (bottom left), 500-fold (bottom middle), and 1000-fold (bottom right).

Compared to the samples were KPS was used as initiator (see Figure 3.30), the individual values of $F_{\max }, E_{\text {porous polymer }}$ and $P$ are closer together when AIBN is used as initiator. All results as well as the average values are summed up in Table 3.6.

Table 3.6: Porosity $P, E_{\text {porous polymer }}, F_{\max }$, and $C_{\mathrm{C}}$ of the samples shown in Figure 3.31 where $\beta_{\text {styrene }}=50 \mathrm{wt} . \%$ and $\beta_{\text {AIBN }}=1.82 \mathrm{wt} . \%$.

\begin{tabular}{|c|c|c|c|c|}
\hline & Porosity $\boldsymbol{P} / \boldsymbol{\%}$ & $\boldsymbol{E}_{\text {porous polymer } / \mathbf{M P a}}$ & $\boldsymbol{F}_{\max } / \mathbf{N}$ & $\boldsymbol{C}_{\mathbf{C}}$ \\
\hline black stress-strain-curve & $77 \pm 1$ & 89 & 30 & $4.55 \pm 0.92$ \\
\hline red stress-strain-curve & $78 \pm 1$ & 97 & 25 & $5.53 \pm 1.12$ \\
\hline green stress-strain-curve & $77 \pm 1$ & 102 & 32 & $5.07 \pm 1.02$ \\
\hline blue stress-strain-curve & $77 \pm 1$ & 114 & 35 & $5.85 \pm 1.18$ \\
\hline average & $\mathbf{7 8} \pm \mathbf{2}$ & $\mathbf{1 0 2} \pm \mathbf{1 3}$ & $\mathbf{3 0} \pm \mathbf{5}$ & $\mathbf{5 . 3} \pm \mathbf{1 . 7}$ \\
\hline
\end{tabular}


In Figure 3.31 (top right), one can see that the red, green, and blue stress-strain-curves closely resemble each other in the linear region. In contrast, the black stress-strain-curve deviates from the other three. The porosity $P=78 \pm 2 \%$ and therefore slightly higher than the ideal $P=74 \%$. This can be explained when looking at Figure 3.31 (bottom right) where it is apparent that the struts of the pore walls have an additional porosity. Moreover, the pores are fairly monodisperse $\left(d_{\text {pore }}=78 \pm 2 \mu \mathrm{m}\right)$, close-packed, and spherical. The values of $E_{\text {porous polymer }}=102 \pm 13 \mathrm{MPa}$ and of $F_{\max }=30 \pm 5 \mathrm{~N}$ are much higher compared to the values of the KPS initiated porous polymers (see Figure 3.30). The calculated average value of $C_{\mathrm{C}}$ is $C_{\mathrm{C}}=5.3 \pm 1.7$ which is much larger than the expected $C_{\mathrm{C}}=1$. The reason for this enormous deviation lies in the values of

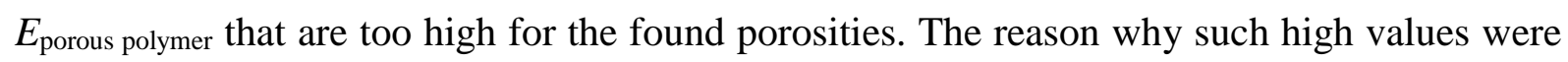

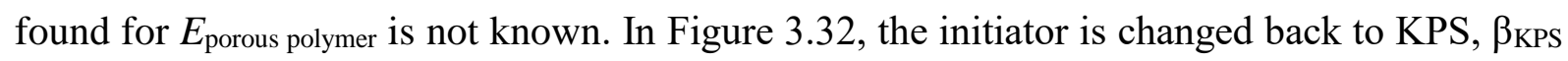
$=2.98 \mathrm{wt} . \%$, and $\beta_{\text {styrene }}$ is increased to $90 \mathrm{wt} . \%$.
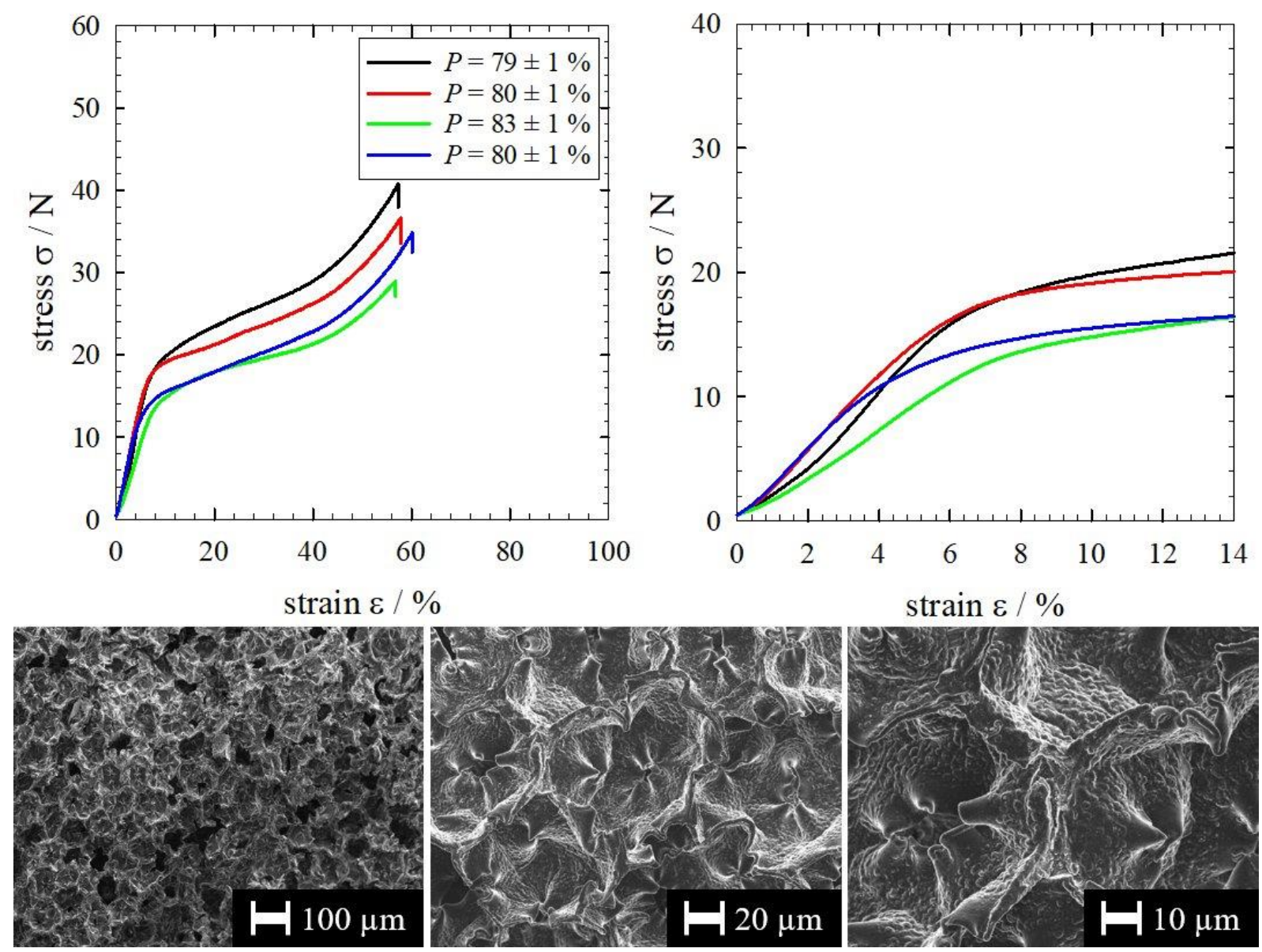

Figure 3.32: (top) Stress-strain-curves and (bottom) SEM pictures of macroporous PS/polyDVB obtained via polymerization of monodisperse water-in-styrene/DVB HIPEs with $\beta_{\text {styrene }}=$ $90 \mathrm{wt} . \%, \beta_{\mathrm{KPS}}=2.98 \mathrm{wt} . \%$, and $\beta_{\text {surfactant }}=10 \mathrm{wt} . \%$. The stress-strain-curves on the top right are magnifications of the stress-strain-curves on the top left at lower strain. The magnifications of the SEM pictures are 100-fold (bottom left), 500-fold (bottom middle), and 1000-fold (bottom right). 
Most strikingly, the stress-strain-curves are elastomeric for $\beta_{\text {styrene }}=90 \mathrm{wt} . \%$ and $\beta_{\mathrm{KPS}}=2.98$ wt. $\%$, while they are elastic-brittle independent of the type of initiator for $\beta_{\text {styrene }}=50 \mathrm{wt} . \%$ (see Figure 3.30 (top) and Figure 3.31 (top)). This can be explained by the change in crosslinking density when $\beta_{\mathrm{DVB}}$ is decreased from $50 \mathrm{wt} . \%$ to $10 \mathrm{wt} . \%$ ( $\beta_{\text {styrene }}$ increased from $50 \mathrm{wt} \%$ to 90 wt. \%). A high crosslinking density as is the case for $\beta_{\mathrm{DVB}}=50 \mathrm{wt} . \%\left(\beta_{\text {styrene }}=50 \mathrm{wt} . \%\right)$ creates a more rigid continuous polymer phase [Wie82]. Therefore, it ruptures at higher strains. At low crosslinking densities, the polymer is more elastic and is thus able to deform at higher strains. Figure 3.32 (bottom) shows that the pores are close-packed and fairly monodisperse $\left(d_{\text {pore }}=79\right.$ $\pm 7 \mu \mathrm{m})^{10}$. The shape of the $2 \mathrm{D}$ pore cross-sections is strongly deformed from the initially circular shape $(S=1.24 \pm 0.09)$. All determined values and the calculated average values are summed up in Table 3.7.

Table 3.7: Porosity $P, E_{\text {porous polymer }}, F_{\max }$, and $C_{\mathrm{C}}$ of the samples shown in Figure 3.32 where $\beta_{\text {styrene }}=90 \mathrm{wt} . \%$ and $\beta_{\mathrm{KPS}}=2.98 \mathrm{wt} . \%$.

\begin{tabular}{|c|c|c|c|c|}
\hline & Porosity $\boldsymbol{P} / \boldsymbol{\%}$ & $\boldsymbol{E}_{\text {porous polymer } / \mathbf{M P a}}$ & $\boldsymbol{F}_{\max } / \mathbf{N}$ & $\boldsymbol{C}_{\mathbf{C}}$ \\
\hline black stress-strain-curve & $79 \pm 1$ & 36 & 20 & $0.90 \pm 0.27$ \\
\hline red stress-strain-curve & $80 \pm 1$ & 36 & 20 & $1.01 \pm 0.30$ \\
\hline green stress-strain-curve & $83 \pm 1$ & 21 & 14 & $0.80 \pm 0.24$ \\
\hline blue stress-strain-curve & $80 \pm 1$ & 31 & 15 & $0.90 \pm 0.27$ \\
\hline average & $\mathbf{8 1} \pm \mathbf{3}$ & $\mathbf{2 9} \pm \mathbf{8}$ & $\mathbf{1 7} \pm \mathbf{3}$ & $\mathbf{0 . 9} \pm \mathbf{0 . 4}$ \\
\hline
\end{tabular}

The calculated average values for $E_{\text {porous polymer }}$ and $F_{\max }$ were $E_{\text {porous polymer }}=29 \pm 8 \mathrm{MPa}$ and $F_{\max }=17 \pm 3 \mathrm{~N}$. A comparison with the values obtained when $\beta_{\text {styrene }}=50 \mathrm{wt} . \%$ is difficult to do since with $\beta_{\text {styrene }}=50 \mathrm{wt}$. $\%$, the calculated values came along with a high uncertainty. The porosity of the inner layer of the pore walls is the reason why $P=81 \pm 3 \%$ and thus higher than the ideal $P=74 \%$. Even if the pores are closed-pore, $C_{\mathrm{C}}$ was again calculated as if they were open-pore (see equation (2.24)) because the necessary parameters $\Phi$ and $v^{*}$ to calculate $C_{1}$ and $C_{1}$ ' are not known (see equation (2.25)). The calculated average value was $C_{\mathrm{C}}=0.9 \pm$ 0.4 and thus closer to the expected value of $C_{\mathrm{C}}=1$. Finally, in Figure 3.33, AIBN was used as initiator, $\beta_{\mathrm{AIBN}}=1.82 \mathrm{wt} . \%$, and at the higher styrene content of $\beta_{\mathrm{styrene}}=90 \mathrm{wt} . \% \mathrm{was}$ used.

\footnotetext{
${ }^{10} d_{\text {pore }}$ was calculated as if the pore cross-section were circular with equation (3.1).
} 

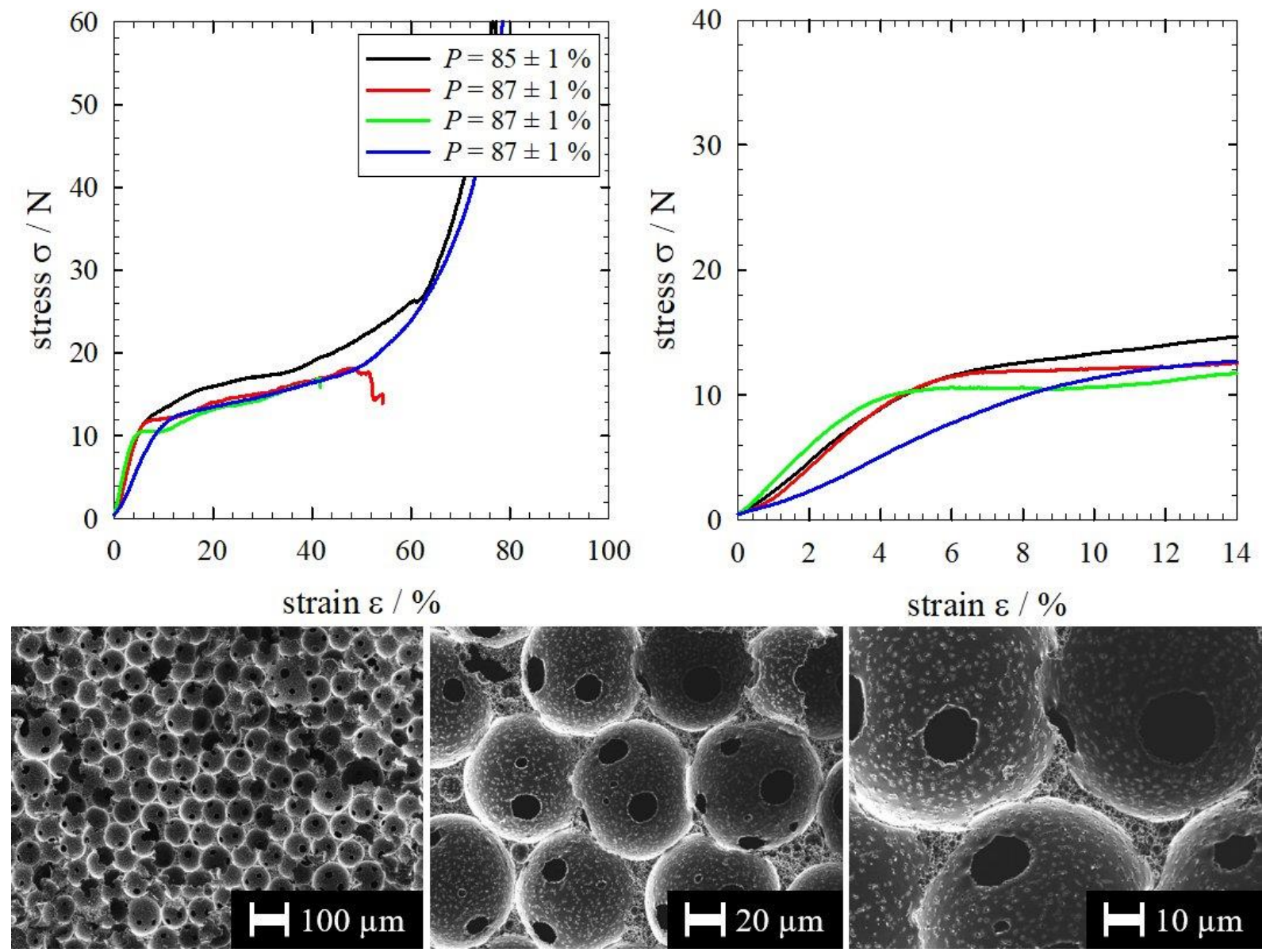

Figure 3.33: (top) Stress-strain curves and (bottom) SEM pictures of macroporous PS/polyDVB obtained via polymerization of monodisperse water-in-styrene/DVB HIPEs with $\beta_{\text {styrene }}=$ $90 \mathrm{wt} . \%, \beta_{\text {AIBN }}=1.82 \mathrm{wt} . \%$, and $\beta_{\text {surfactant }}=10 \mathrm{wt} . \%$. The stress-strain-curves on the top right are magnifications of the stress-strain-curves on the top left at lower strain. The magnifications of the SEM pictures are 100-fold (bottom left), 500-fold (bottom middle), and 1000-fold (bottom right).

Similar to when KPS was used as initiator and $\beta_{\text {styrene }}=90 \mathrm{wt} . \%$ (see Figure 3.32), the stressstrain-curves are also elastomeric when AIBN is used as initiator. In the SEM pictures in Figure 3.33 (bottom), the pores appear fairly monodisperse $\left(d_{\text {pore }}=78 \pm 5 \mu \mathrm{m}\right)$, close-packed, and

Table 3.8: Porosity $P, E_{\text {porous polymer }}, F_{\max }$, and $C_{\mathrm{C}}$ of the samples shown in Figure 3.33 where $\beta_{\text {styrene }}=90 \mathrm{wt} . \%$ and $\beta_{\mathrm{AIBN}}=1.82 \mathrm{wt} . \%$.

\begin{tabular}{|c|c|c|c|c|}
\hline & Porosity $\boldsymbol{P}$ & $\boldsymbol{E}_{\text {porous polymer }} / \mathbf{M P a}$ & $\boldsymbol{F}_{\max } / \mathbf{N}$ & $\boldsymbol{C}_{\mathbf{C}}$ \\
\hline black stress-strain-curve & $86 \pm 1$ & 25 & 12 & $1.30 \pm 0.39$ \\
\hline red stress-strain-curve & $87 \pm 1$ & 27 & 12 & $1.68 \pm 0.50$ \\
\hline green stress-strain-curve & $87 \pm 1$ & 31 & 10 & $2.00 \pm 0.60$ \\
\hline blue stress-strain-curve & $87 \pm 1$ & 12 & 12 & $0.77 \pm 0.24$ \\
\hline average & $\mathbf{8 7} \pm \mathbf{2}$ & $\mathbf{2 2} \pm \mathbf{1 0}$ & $\mathbf{1 1} \pm \mathbf{1}$ & $\mathbf{1 . 6} \pm \mathbf{1 . 1}$ \\
\hline
\end{tabular}


spherical. All determined values and the calculated average values are summed up in Table 3.8. The measured porosity was $P=87 \pm 1 \%$ and thus much higher than the expected $P=74 \%$. This implies that the continuous polymer phase has a relatively high additional porosity. The calculated average values for $E_{\text {porous polymer }}$ and $F_{\max }$ were $E_{\text {porous polymer }}=22 \pm 10 \mathrm{MPa}$ and $F_{\max }$ $=11 \pm 1 \mathrm{~N}$. Compared to when $\beta_{\text {styrene }}=50 \mathrm{wt}$. $\%$ (see Figure 3.31), $E_{\text {porous polymer }}$ and $F_{\max }$ are much lower. On the one hand, this could be expected since the porosity is higher for $\beta_{\text {styrene }}=$ $90 \mathrm{wt} . \%$ compared to $\beta_{\text {styrene }}=50 \mathrm{wt} . \%$. On the other hand, the crosslinking density is also lower for $\beta_{\text {styrene }}=90 \mathrm{wt} . \%\left(\beta_{\mathrm{DVB}}=10 \mathrm{wt} . \%\right)$ compared to $\beta_{\text {styrene }}=50 \mathrm{wt} . \%\left(\beta_{\mathrm{DVB}}=50 \mathrm{wt} . \%\right)$. Thus, the polymer matrix is less rigid for $\beta_{\text {styrene }}=90 \mathrm{wt} . \%\left(\beta_{\mathrm{DVB}}=10 \mathrm{wt} . \%\right)$ and consequently, $E_{\text {porous }}$ polymer and $F_{\max }$ are smaller. The calculated average value for $C_{\mathrm{C}}$ was $C_{\mathrm{C}}=1.6 \pm 1.1$ which is also lower than when $\beta_{\text {styrene }}=50 \mathrm{wt}$. $\%$, but still higher than the expected $C_{\mathrm{C}}=1$. Compared to when KPS was used as initiator (see Figure 3.32), $E_{\text {porous polymer }}$ and $F_{\max }$ are slightly smaller when AIBN was used as initiator. Though, the porosity is higher when AIBN was used as initiator. When the porosity is included for the calculation of $C_{\mathrm{C}}, C_{\mathrm{C}}$ is higher when AIBN was used as initiator. This suggests that the close-packed spherical morphology found when AIBN is used as initiator is stiffer than the disordered, polyhedral morphology found when KPS is used as initiator.

Summarized, the stress-strain-curves are elastomeric for $\beta_{\text {styrene }}=90 \mathrm{wt} . \%$ and elastic-brittle for $\beta_{\text {styrene }}=50 \mathrm{wt} . \%$. This is independent of the type of initiator. The stress-strain-curves are also elastic-brittle when $\beta_{\text {styrene }}=70 \mathrm{wt} . \%,=30 \mathrm{wt} . \%$, and $=10 \mathrm{wt} . \%$. They are not shown here, but are similar to the stress-strain-curves obtained for $\beta_{\text {styrene }}=50 \mathrm{wt} . \%$. When AIBN is used as

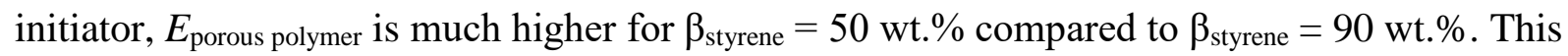
is still true when the higher porosities at $\beta_{\text {styrene }}=90 \mathrm{wt} . \%$ are considered. For $\beta_{\text {styrene }}=50 \mathrm{wt} . \%$

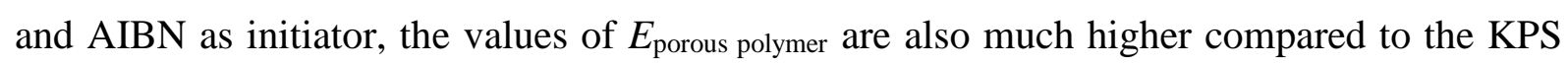
initiated porous polymers. The reason for this is not known. When KPS is used as initiator, no such trend could be observed since the values were too far spread for $\beta_{\text {styrene }}=50 \mathrm{wt}$. $\%$. For $\beta_{\text {styrene }}=90 \mathrm{wt} . \%$, it appears as if the close-packed spherical morphology obtained with AIBN as initiator is stiffer than the disordered, polyhedral morphology with KPS as initiator. The summary of all average values is presented in Table 3.9. 
Table 3.9: Type of initiator, $\beta_{\text {styrene, }}$, porosity $P, E_{\text {porous polymer, }} F_{\max }$, and $C_{\mathrm{C}}$ of the sample whose results were shown in Section 3.3.

\begin{tabular}{|c|c|c|c|c|c|}
\hline $\begin{array}{c}\text { type of } \\
\text { initiator }\end{array}$ & $\begin{array}{c}\boldsymbol{\beta}_{\text {styrene }} / \\
\mathbf{w t .} \%\end{array}$ & $\begin{array}{c}\text { Porosity } \boldsymbol{P} / \\
\boldsymbol{\%}\end{array}$ & $\begin{array}{c}\boldsymbol{E}_{\text {porous polymer }} / \\
\mathbf{M P a}\end{array}$ & $\boldsymbol{F}_{\max } / \mathbf{N}$ & $\boldsymbol{C}_{\mathbf{C}}$ \\
\hline KPS & 50 & $73 \pm 4$ & $51 \pm 36$ & $29 \pm 21$ & $1.9 \pm 1.2$ \\
\hline AIBN & 50 & $78 \pm 2$ & $102 \pm 13$ & $30 \pm 5$ & $5.3 \pm 1.7$ \\
\hline KPS & 90 & $81 \pm 3$ & $29 \pm 8$ & $17 \pm 3$ & $0.9 \pm 0.4$ \\
\hline AIBN & 90 & $87 \pm 2$ & $22 \pm 10$ & $11 \pm 1$ & $1.6 \pm 1.1$ \\
\hline
\end{tabular}

When $\beta_{\text {initiator }}$ or $\beta_{\text {surfactant }}$ were varied, no significant trends could be found. The stress-strain-

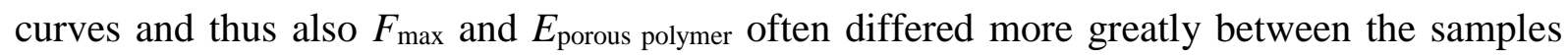
within one composition than between two compositions. The same is true when one searched for differences between monodisperse macroporous PS/polyDVB and polydisperse macroporous PS/polyDVB. While differing porosities were sometimes the explanation for the differences within one composition, they cannot explain all observations. As above-mentioned, no direct structure-property-relationships could be determined since the morphology was determined with SEM from one sample, while the stress-strain-curves were recorded from other samples with the same composition. Additionally, with SEM only a small part of the whole sample can be looked at. While the sample height is $\sim 7 \mathrm{~mm}(7000 \mu \mathrm{m})$, with SEM the morphology of only 1-2 layers can be visualized $(\sim 80-160 \mu \mathrm{m})$. Thus, SEM does not provide the structure of the whole sample which is why structure-property-relationships are difficult to draw from the combination of SEM and stress-strain-curves. Overall, it was therefore not possible to examine how small changes in the morphology affect the mechanical properties. The potentially existing differences could not be detected with the method used in this $\mathrm{PhD}$ thesis. The solution to this problem would be the use of micro-computed tomography $(\mu-\mathrm{CT})$ while compressing the samples. Thus, both the stress-strain-curve and the morphology of the whole sample could be determined simultaneously. However, this combination requires a lot of time and was moreover not available for this $\mathrm{PhD}$ thesis. Determining the morphology with $\mu$ CT while simultaneously measuring the mechanical properties could be the focus of future research. 
-94 - 


\section{Conclusions and Outlook}

\subsection{Conclusion}

\section{Starting Situation}

Using microfluidics, it is possible to generate monodisperse water-in-styrene/divinylbenzene (DVB) emulsions. Because the water droplets have a uniform size and a higher density than the surrounding monomer phase, their sedimentation results in a high internal phase emulsion (HIPE) with a water volume fraction of $\Phi_{\text {disp }}=74 \mathrm{vol} \%$. By polymerizing and drying the HIPE, its structure is retained and monodisperse porous polystyrene (PS)/polydivinylbenzene (polyDVB) is produced. Because the average pore diameter is $\sim 80 \mu \mathrm{m}$ and thus much larger than $50 \mathrm{~nm}$, the material is classified as macroporous [Eve72, Bur76]. The morphology of monodisperse macroporous PS/polyDVB strongly depends on which type of initiator is used. With monomer-soluble azobisisobutyronitrile (AIBN), the pores are spherical and interconnected and the pore walls exhibit an additional porosity (see Figure 4.1 (left)). With water-soluble potassium peroxydisulfate (KPS), on the other hand, the pores are polyhedral and not interconnected and the pores walls are comprised of two similar looking outer layers and one inner layer (see Figure 4.1 (right)). Thus, two questions arise: (1) Firstly, why are spherical droplets transforming into polyhedral pores during the polymerization? This requires a certain mechanism to increase the area of the water/monomer interface and thus counteract capillary pressure. (2) Secondly, why are the pore walls made up of three layer?

\section{Hypothesis 1: Osmotic Transport of DVB}

In previous works, Quell et al. [Que16b, Que17a] answered both questions with a mechanism that revolves around osmotic transport of DVB (see Figure 4.2 (right)). When the polymerization starts at the interface because a water-soluble initiator is used, a polyDVB-rich layer forms close to the interface since DVB polymerizes faster than styrene [Sch75] (see Figure 4.2 (right), purple layer). This induces a concentration gradient of DVB between the films where DVB has been depleted and the Plateau borders where the DVB concentration is still at its initial value. As a consequence, osmotic transport of DVB from the Plateau borders into the films thins out the Plateau borders and increases the thickness of the films (see Figure 4.2 (right), red arrows). Overall, this leads to a change of the shape of the interface from spherical to polyhedral. The three layers of the pore walls were explained in that the two outer layers are 


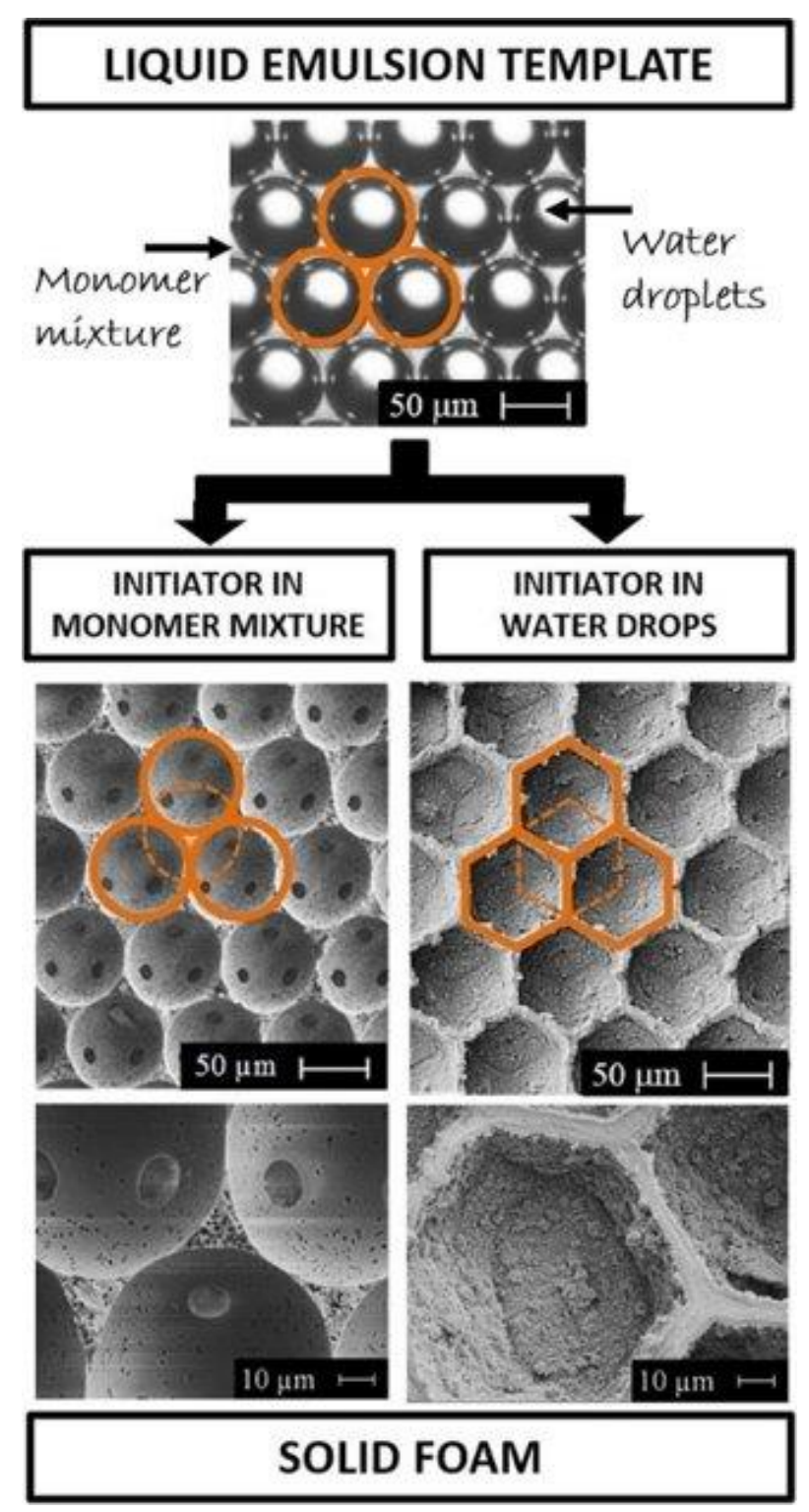

Figure 4.1: Polymerization and drying of a (top) monodisperse water-in-styrene/DVB HIPE leads to (left) open-pore monodisperse macroporous PS/polyDVB with spherical pores and porous pore walls when monomer-soluble AIBN is used and (right) closed-pore monodisperse macroporous PS/polyDVB with polyhedral pores and three-layered pore walls when watersoluble KPS is used. This Figure was copied from [Que17a]. License number 5004850663340.

polyDVB-rich, while the inner layer is PS-rich because of the slower polymerization of styrene (see Figure 4.2 (right), yellow layer). Though Quell et al. found experimental support of this hypothesis [Que17a], a satisfying and quantitative proof was still missing. Thus, this $\mathrm{PhD}$ thesis' goal was to either prove or disprove the above-explained hypothesis. This was tackled by drawing up logical deductions about what ought to happen to the morphology of monodisperse macroporous PS/polyDVB when the concentration of a component is varied systematically (see Section 3.1). If the experimental results support the existing hypothesis, a quantitative description of it might become feasible from the large number of new findings. If, 


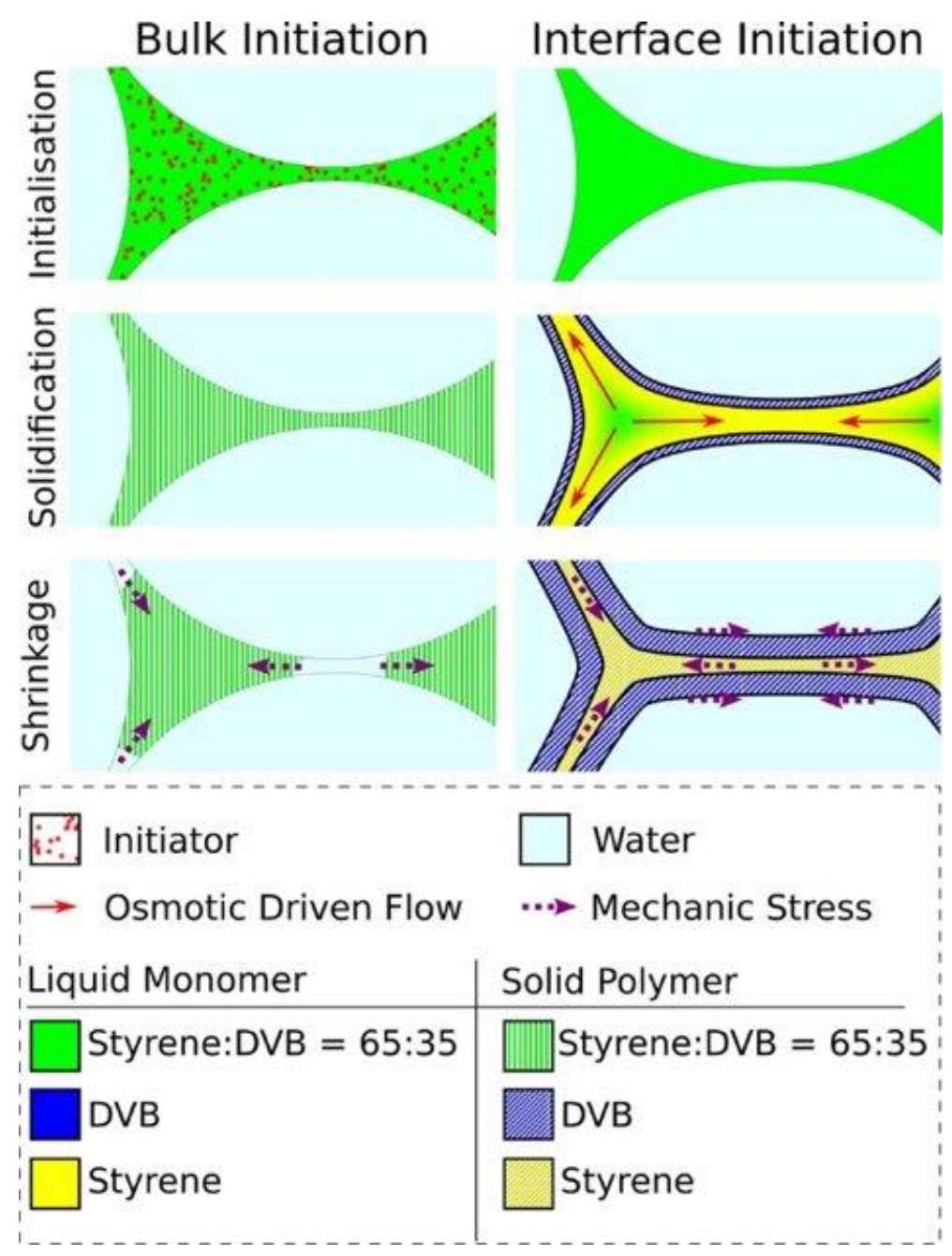

Figure 4.2: Explanation of the morphologies depicted in Figure 4.1 as described by [Que17a]. (Left) With monomer-soluble AIBN, the polymerization is occurring homogeneously throughout the continuous phase and the droplet shape is retained. The shrinkage of the continuous phase during polymerization causes mechanic stress and a consequent film rupture. (Right) With water-soluble KPS, the polymerization startes locally at the water/monomer interface and a polyDVB-rich layer is formed. The depletion of DVB in the films is balanced by osmotic-driven flow of DVB from the Plateau borders into the films. This induces a deformation of the droplet shape from spherical to polyhedral. The shrinkage of the continuous phase during polymerization causes internal mechanic stress which wrinkles the polyDVB-rich outer layer. This Figure was copied from [Que17a]. License number 5004850663340.

on the other hand, the experimental results contradict the existing hypothesis, it has to be rejected and a new explanation has to be worked out. (1) Firstly, the styrene mass fraction $\beta_{\text {styrene }}$ was varied between the initial $50 \mathrm{wt} \%$ (styrene/DVB mass ratio 50/50) and $90 \mathrm{wt} \%$ (styrene/DVB mass ratio 90/10) in steps of $10 \mathrm{wt} . \%$ (see Section 3.1.1). When the monomer composition is increased towards pure styrene, (1a) the extent of osmotic transport of DVB ought to decrease. As a consequence, the mechanism that transforms the shape of the pores ceases and thus, the pores ought to remain spherical. However, when $\beta_{\text {styrene }}$ was varied, scanning electron microscopy (SEM) pictures did not support the expectations. In fact, the pores 
became even more distorted from the spherical shape when $\beta_{\text {styrene }}$ was increased from $50 \mathrm{wt} . \%$ to $90 \mathrm{wt} . \%$. To quantify this observation, a so-called shape factor $S$ was introduced where the circumference $C$ of a $2 \mathrm{D}$ pore cross-section is divided by the square root of its area $A$. Thus, $S$ is lowest for a circular shape and increases with increasing distortion from the circular shape. In the samples, $S$ increased from values found for hexagons at $\beta_{\text {styrene }}=50 \mathrm{wt} . \%$ to much higher values at $\beta_{\text {styrene }}=90 \mathrm{wt} . \%$, thus indeed quantifying the SEM observations. When $\beta_{\text {styrene was }}$ decreased from $50 \mathrm{wt} . \%$ to $10 \mathrm{wt} . \%$, the shape of the $2 \mathrm{D}$ pore cross-sections ought to become circular as well if one assumes osmotic transport of DVB. However, the shape of the pore crosssections remained hexagonal. Therefore, the development of the pore shape as function of $\beta_{\text {styrene }}$ is the first counterargument to osmotic transport of DVB. Apart from the shape of the pores, changing $\beta_{\text {styrene }}$ also ought to have an influence on the thickness of the layers in the pore walls. When $\beta_{\text {styrene }}$ is increased from $50 \mathrm{wt} \%$ to $90 \mathrm{wt} . \%$, (1b) the thickness of the supposedly polyDVB-rich outer layers ought to decrease and the thickness of the supposedly PS-rich inner layer ought to increase. However, the expectations were not supported here either. The pore

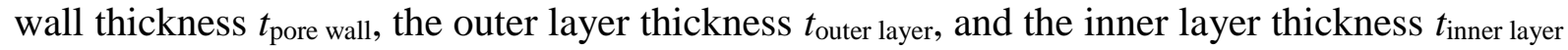
all decreased when $\beta_{\text {styrene }}$ was increased from 50 wt.\% to 90 wt.\%. Thus, the relative thicknesses of the outer and inner layer remained constant as function of $\beta_{\text {styrene }}$ which serves as the second counterargument to osmotic transport of DVB. When AIBN was used as initiator for the sake of comparison, the morphology did not change when $\beta_{\text {styrene }}$ was varied.

(2) Secondly, the KPS mass fraction $\beta_{\text {KPS }}$ was changed (see Section 3.1.2). The reasoning behind was that an increase of the amount of KPS increases the polymerization rate. Thus, the comparatively slow diffusion process of DVB from the Plateau borders into the films has less time to occur. Consequently, the pores ought to remain more spherical. However, when $\beta_{\mathrm{KPS}}$ was increased, the hexagonal shape of the $2 \mathrm{D}$ pore cross-sections became slightly more distorted. This observation was quantified by the shape factor $S$. Therefore, the development of the pore shape as function of $\beta_{\mathrm{KPS}}$ is the third counterargument against osmotic transport of DVB. The measurement of $t_{\text {pore wall }}, t_{\text {outer layer, }}$ and $t_{\text {inner layer }}$ as function of $\beta_{\mathrm{KPS}}$ revealed that the thicknesses are independent of $\beta_{\text {KPS. }}$. When KPS was substituted by AIBN for the sake of comparison, the morphology did not change when $\beta_{\text {AIBN }}$ was varied. The results of Section 3.1 were published in a paper in Colloid and Polymer Science [Koc20]. 


\section{Hypothesis 2: Surfactant Diffusion}

Overall, the variation of $\beta_{\text {styrene }}$ and $\beta_{\text {KPS }}$ demonstrated that osmotic transport of DVB does not exist. Thus, the new goal of this $\mathrm{PhD}$ thesis was to find the real mechanism that answers (1) why spherical droplets become polyhedral pores and (2) why the pore walls are comprised of three layers (see Section 3.2.1). As a first step, the surfactant mass fraction $\beta_{\text {surfactant }}$ was varied between $5-20 \mathrm{wt} . \%$ in steps of $5 \mathrm{wt} . \%$. In the 2D pore cross-sections of the SEM pictures, the shape was always hexagonal. This means that the mechanism that increases the interfacial area during polymerization is independent of $\beta_{\text {surfactant. To determine if and how the size of the layers }}$ in the pore walls change when $\beta_{\text {surfactant }}$ is varied, their areas $A_{\text {outer layer }}$ and $A_{\text {inner layer }}$ as well as the total area of the pore wall $A_{\text {pore wall }}$ were measured. It was found that $A_{\text {pore wall }}$ and $A_{\text {outer layer }}$ decrease as function of $\beta_{\text {surfactant, while }} A_{\text {inner layer }}$ remains constant. To determine how $A_{\text {outer layer }}$ and $A_{\text {inner layer }}$ develop independent of $A_{\text {pore wall, }}$ the relative area of the outer layer $A_{\text {outer layer }} / A_{\text {pore }}$ wall and the relative area of the inner layer $A_{\text {inner layer }} / A_{\text {pore wall }}$ were calculated. Here, a clear trend was observed: The relative area of the outer layer decreases as function of $\beta_{\text {surfactant, }}$ while the relative area of the inner layer increases. This finding indicates that the formation of the layers is linked to the surfactant. SEM pictures at higher magnifications revealed that the outer layers are non-porous, while the inner layer is porous. This implies that the porosity of the inner layer is caused by the surfactant since the relative area of the porous inner layer increases with increasing $\beta_{\text {surfactant. }}$

The intended use of the surfactant is to stabilize the water/monomer interface. However, with the help of an educated guess for the head group area $A_{\text {head group }}$ of the surfactant [Wet01, Dom07], the amount of surfactant actually necessary to cover the interface $\beta_{\text {surfactant at interface }}$ was calculated. This value is below $0.1 \mathrm{wt} . \%$ which means that the majority of the surfactant is dissolved in the continuous phase. Thus, the surfactant can cause a porosity in the pore walls when it is removed after the polymerization. However, this opens up the question why there is only a porous inner layer and not a porous pore wall. To investigate what happens to the surfactant during the polymerization, a model ternary phase diagram consisting of styrene, surfactant, and PS was used to simulate the polymerization. Here, surfactant/styrene mixtures simulated different $\beta_{\text {surfactant }}$ and the styrene/PS ratio simulated the polymerization. When PS was added and dissolved in the surfactant/styrene mixtures, the initially clear mixtures eventually separated into two phases. When $\beta_{\text {surfactant }}$ was low, high amounts of PS could be dissolved, while phase separation occurred much earlier when $\beta_{\text {surfactant }}$ was high. This means that the surfactant is neither soluble in PS nor in partially polymerized styrene/PS mixtures. Finally, it 
was investigated whether the porosity of the inner layer is solely caused by the surfactant or if the surfactant forms small water droplets that add to the porosity. In experiments where monomer phases were placed over water, the initially clear monomer phases became turbid and white over time. Thus, small water droplets surrounded by surfactant formed and grew in size over time. Small angle neutron scattering, freeze fracture electron microscopy, and dynamic light scattering experiments showed that the surfactant already forms structures (inverse micelles) in the low nanometer range without water. However, to be able to grow in size, these structures require water and thus turn into small water droplets by incorporating water. Therefore, the porosity of the inner layer stems from both surfactant and small water droplets.

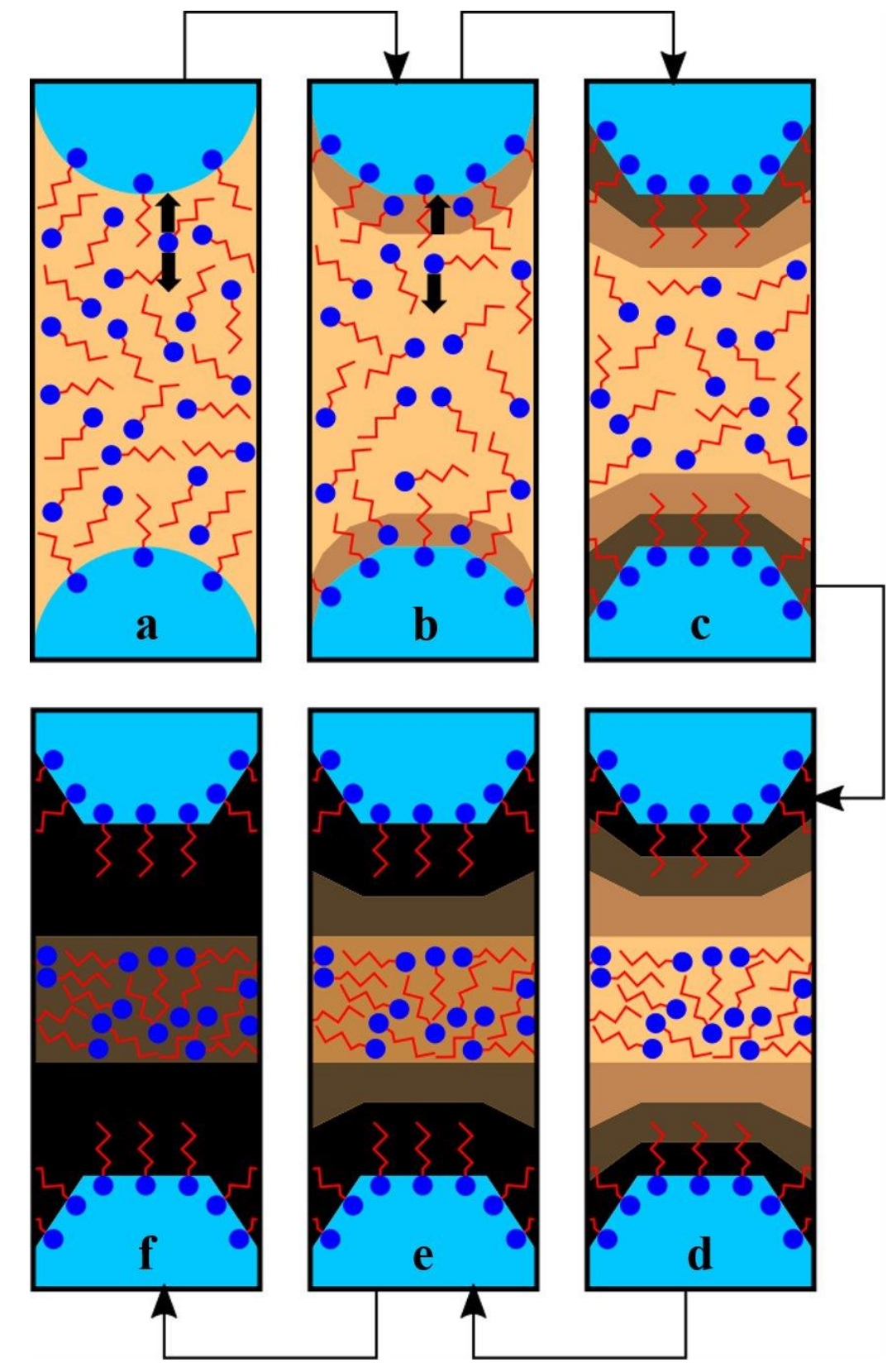

Figure 4.3: Schematic drawing of the mechanism that increases the area of the water/monomer interface and that creates non-porous outer layers and a porous inner layer in the pore wall. 
From all results, a mechanism that answers (1) why spherical droplets become polyhedral pores and (2) why the pore walls are comprised of three layers was concluded (see Section 3.2.2). When the polymerization starts at the water/monomer interface because water-soluble KPS is used, a small and partially polymerized layer forms close to the interface (see Figure 4.3 (b)). Since the surfactant is not soluble in this layer, it diffuses away from there. One option is to diffuse to the nearby water/monomer interface, where the surfactant overpopulates the interface which causes an increase of the interface [Jia99, Pas 12, Giu17]. This increase changes the shape of the interface from spherical to polyhedral (see Figure $4.3(\mathrm{a} \rightarrow \mathrm{b} \rightarrow \mathrm{c})$ ). Another option for the surfactant is to diffuse into the interior of the continuous phase. Thus, the surfactant accumulates in the interior of the continuous phase, while the regions close to the interface become surfactant-free (see Figure $4.3(\mathrm{~b} \rightarrow \mathrm{c} \rightarrow \mathrm{d})$ ). When the macroporous material is purified after the polymerization, the surfactant is washed out, which creates the porosity of the inner layer. In turn, the outer layers remain non-porous. Since the small water droplets are surrounded by surfactant, they diffuse along with the surfactant. Thus, they either diffuse back into the dispersed phase or remain in the interior of the continuous phase. There, they add to the porosity of the inner layer when they are washed and dried out. The results of Section 3.2 were published in a paper in the Journal of Colloid and Interface Science [Koc21].

Another focus of this $\mathrm{PhD}$ thesis was the investigation of the mechanical properties of monodisperse macroporous PS/polyDVB (see Section 3.3). The influence of the type and amount of initiator, of the styrene/DVB mass ratio, of the amount of surfactant, and of the change between mono- and polydispersity were investigated. It was found that for $\beta_{\text {styrene }}=90$ wt. $\%$ the samples were elastomeric, while they were elastic-brittle for $\beta_{\text {styrene }} \leq 70 \mathrm{wt} \%$. No further significant trends could be found because it was not possible to determine the morphology of a whole sample and to also record the mechanical properties of that sample. As a consequence, it was impossible to obtain structure-property-relationships.

\subsection{Outlook}

To solve the just-mentioned problem for the determination of structure-property-relationships, a combination of methods that simultaneously investigate the morphology of a whole sample and determine the mechanical properties has to be used. Instead of only examining 1-2 layers of a sample with SEM, with micro-computed tomography ( $\mu$-CT) the morphology of a whole sample can be determined. Therefore, if $\mu-\mathrm{CT}$ is coupled to a universal testing machine, the determination of structure-property-relationships becomes possible. Thierry Roland and the 
work group of Wiebke Drenckhan have such a piece of equipment at their disposal at the Institut Charles Sadron (ICS) in Strasbourg. Thus, by cooperating with them, the mechanical properties of monodisperse and polydisperse macroporous PS/polyDVB can be recorded and differentiated. The only drawback of $\mu-\mathrm{CT}$ is that it takes quite a long time to record one sample which is why only selected samples can be investigated.

The use of $\mu$-CT could also help with two smaller issues concerning the transformation of spherical droplets into polyhedral pores. Firstly, the mechanism shown in Figure 4.3 suggests that the transformation of the shape happens in the early stages of the polymerization. At this point, the interface is already overpopulated with surfactant, but the continuous phase is only partially polymerized and can thus still comply with the change of the shape. By monitoring the polymerization with $\mu$-CT, this assumption can be tested. Secondly, it has not been clarified yet why the pore walls between two pores are much thicker than they were initially between two droplets. This means that during the polymerization, material diffuses into the films. Monitoring the polymerization with $\mu$-CT could help investigating this question.

Another aspect that has to be improved is the output rate at which monodisperse macroporous PS/polyDVB can be produced. For this, two options exist: On the one hand, an upscaling of the microfluidic process would result in higher amounts of monodisperse HIPEs while maintaining a very high monodispersity. An example of how the output of microfluidics can be increased was shown by Nisisako et al. [Nis12] who developed a microfluidic parallelization chip. In this device, an output rate of $180 \mathrm{~mL} \mathrm{~h}^{-1}$ is enabled by having parallel droplet generation in up to 144 constrictions. The workgroup of Wiebke Drenckhan at the ICS in Strasbourg built such a parallelization device where preliminary tests have already been undertaken. On the other hand, the output rate can be drastically increased to several $\mathrm{mL} \mathrm{min}^{-1}$ with membrane emulsification. However, this is accompanied by a loss in monodispersity. Nevertheless, the generated droplets are still far from being polydisperse with PDIs between $10-26 \%$ [Kos05, Egi08, Gas08, Hol10] and the average droplet size can also still be controlled. Thus, if it turns out that the mechanical properties of monodisperse macroporous PS/polyDVB are not better compared to those of polydisperse ones, membrane emulsification offers a promising alternative. 


\section{Experimental}

\subsection{Materials}

Styrene (99\%), divinylbenzene (DVB, technical grade $80 \%$, residual $20 \%$ is mostly ethylstyrene), potassium peroxydisulfate (KPS, 99\%), ammonium peroxydisulfate (APS, 99\%), azobisisobutyronitrile (AIBN, 99\%), and polystyrene (PS, average $M_{\mathrm{W}}=35000 \mathrm{~g} \mathrm{~mol}^{-1}$ ) were purchased from Sigma-Aldrich. For the SANS measurements, deuterated Styrene (98\%) was purchased from Cambridge Isotopes and $\mathrm{D}_{2} \mathrm{O}(>99 \%)$ was purchased from Eurisotop. Double distilled water was produced with a Bi $18 E$ distillery from $Q C S$. The technical, non-ionic surfactant Hypermer 2296 was kindly supplied by Croda. The surfactant primarily consists of polyisobutylene succinic anhydride sorbitan esters [Gra13]. All chemicals were used without further purification.

\subsection{Synthesis of Macroporous PS/polyDVB}

The continuous monomer phases consisted of styrene, DVB, and surfactant. The styrene mass

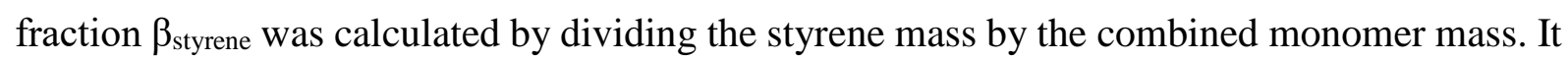
holds

$$
\beta_{\text {styrene }}=\frac{m(\text { styrene })}{m(\text { styrene })+m(\text { DVB })} \cdot 100 \%
$$

The surfactant mass fraction $\beta_{\text {surfactant }}$ was calculated by dividing the surfactant mass by the combined monomer mass. It holds

$$
\beta_{\text {surfactant }}=\frac{m(\text { surfactant })}{m(\text { styrene })+m(\mathrm{DVB})} \cdot 100 \%
$$

If AIBN was added to the continuous phase, the AIBN mass fraction $\beta_{\mathrm{AIBN}}$ was calculated by dividing the AIBN mass by the combined monomer mass. It holds

$$
\beta_{\mathrm{AIBN}}=\frac{m(\mathrm{AIBN})}{m(\text { styrene })+m(\mathrm{DVB})} \cdot 100 \% .
$$

The combined monomer mass was kept constant at $10 \mathrm{~g}$. In the dispersed phase, the $\mathrm{H}_{2} \mathrm{O}$ mass was likewise kept constant at $10 \mathrm{~g}$. This was done to have similar conditions in the microfluidic chip for droplet generation. If KPS was added to the dispersed phase, the KPS mass fraction

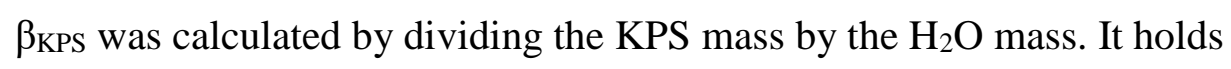




$$
\beta_{\mathrm{KPS}}=\frac{m(\mathrm{KPS})}{m\left(\mathrm{H}_{2} \mathrm{O}\right)} \cdot 100 \%
$$

To better illustrate how the weighed in masses relate to the above-mentioned mass fractions, two exemplary samples are shown in Table 5.1. These are the standard samples with either KPS or AIBN as initiator from which $\beta_{\text {styrene, }} \beta_{\text {initiator, }}$ or $\beta_{\text {surfactant }}$ was varied. For KPS as initiator, the initial values are $\beta_{\text {styrene }}=50 \mathrm{wt} . \%, \beta_{\mathrm{KPS}}=2.98 \mathrm{wt} . \%$, and $\beta_{\text {surfactant }}=10 \mathrm{wt}$. $\%$. For AIBN as initiator, $\beta_{\mathrm{AIBN}}=1.82 \mathrm{wt} . \%$, while $\beta_{\text {styrene }}$ and $\beta_{\text {surfactant }}$ are kept constant.

Table 5.1: Type of initiator, $m$ (styrene), $m$ (DVB), $\beta_{\text {styrene, }} m$ (initiator), $\beta_{\text {initiator }}, m$ (surfactant),

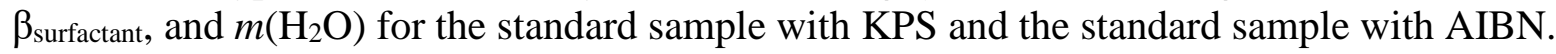

\begin{tabular}{|c|c|c|}
\hline & standard sample with KPS & standard sample with AIBN \\
\hline type of initiator & $\mathrm{KPS}$ & $\mathrm{AIBN}$ \\
\hline$m$ (styrene) & $5 \mathrm{~g}$ & $5 \mathrm{~g}$ \\
\hline$m(\mathrm{DVB})$ & $5 \mathrm{~g}$ & $5 \mathrm{~g}$ \\
\hline$\beta_{\text {styrene }}$ & $50 \mathrm{wt} . \%$ & $50 \mathrm{wt} . \%$ \\
\hline$m$ (initiator) & $0.298 \mathrm{~g}$ & $0.182 \mathrm{~g}$ \\
\hline$\beta_{\text {initiator }}$ & $2.98 \mathrm{wt} . \%$ & $1.82 \mathrm{wt} . \%$ \\
\hline$m($ surfactant $)$ & $1 \mathrm{~g}$ & $1 \mathrm{~g}$ \\
\hline$\beta_{\text {surfactant }}$ & $10 \mathrm{wt} . \%$ & $10 \mathrm{wt} . \%$ \\
\hline $\mathrm{m}\left(\mathrm{H}_{2} \mathrm{O}\right)$ & $10 \mathrm{~g}$ & $10 \mathrm{~g}$ \\
\hline
\end{tabular}

Both the dispersed and the continuous phase were prepared by weighing in all components with a high precision scale (accuracy $0.0001 \mathrm{~g}$ ) into $100 \mathrm{~mL}$ screw top DURAN® borosilicate containers from Schott. By carefully shaking the containers, a homogeneous mixture was obtained. The dispersed and the continuous phase were then connected with the pressure controller $O B$ I MkII (maximum pressure 2 bar, software control ESI) from Elveflow with DURAN® multipart connector caps and Tygon tubes. The two phases were furthermore connected with the microfluidic chip with FEP (fluorinated ethylene propylene) tubing (inner diameter $0.5 \mathrm{~mm}$ ). The continuous phase was additionally split into two channels with a $T$ connector (ethylene tetrafluoroethylene (ETFE), $0.5 \mathrm{~mm}$ ). The microfluidic chip consisted of quartz glass and was purchased from Dolomite. It had a hydrophobic coating, a constriction size of $100 \times 105 \mu \mathrm{m}$, and flow-focussing geometry. The microfluidic chip was embedded into a $H$-interface from Dolomite and connected to the sample vial with FEP tubing (inner diameter $0.5 \mathrm{~mm}$ ). Monodisperse droplet generation was achieved by adjusting the pressures of both phases such that the droplets formed in the dripping regime. For monodisperse droplet generation, the two pressures were kept constant, while for polydisperse droplet generation, a sinusoidal pressure profile was used for the dispersed phase [And18, Dab20]. The droplet 
generation was monitored with a Nikon SMZ - $800 \mathrm{~N}$ optical microscope from Nikon coupled to an Optronis CL600X2 high speed camera from Stemmer Imaging.

Before collecting the emulsion in the sample vial, the average droplet diameter $d_{\text {droplet }}$ was determined. If necessary, the pressures were adjusted such that the desired $d_{\text {droplet }}$ was obtained. The emulsion was poured onto a glass disk and examined with the optical microscope. A picture of the emulsion was taken and processed with the free of charge image processing software ImageJ from National Institutes of Health (NIH, USA). The picture was converted into a binary picture which allowed ImageJ to automatically determine the area $A_{\text {droplet }}$ of each droplet crosssection. From this value, $d_{\text {droplet }}$ and its standard deviation was calculated. Next, the emulsion was collected in a vial until the desired amount was reached. Depending on vial size, this took roughly between 30 - 90 min. During collection, the droplets already sedimented in the vial and thus formed a high internal phase emulsion (HIPE) with $\Phi_{\text {disp }}=74 \mathrm{vol} \%$ at the bottom of the vial. After the collection was finished, complete sedimentation of all droplets was achieved by waiting $\sim 30 \mathrm{~min}$. The excess continuous monomer phase was removed with a syringe. The vial was sealed with a cap, though air holes were pierced into the caps to allow pressure equalization. Then, the vial was placed into an oil bath at $70{ }^{\circ} \mathrm{C}$. The HIPE was polymerized for $48 \mathrm{~h}$ (KPS as initiator) or $24 \mathrm{~h}$ (AIBN as initiator). After polymerization, the samples were removed from the vial with a bench vise and then purified with Soxhlet extraction for at least $12 \mathrm{~h}$ with ethanol as solvent. Finally, the samples were dried in a drying oven at $60{ }^{\circ} \mathrm{C}$ for $72 \mathrm{~h}$.

\subsection{Analysis of Macroporous PS/polyDVB}

\subsubsection{Analysis of Morphology}

The morphology of the samples was examined with scanning electron microscopy (SEM). The samples had to be cut open with a scalpel. To facilitate cutting and ideally have a cut parallel to one layer of pores, the samples were cooled with liquid nitrogen. Pieces of $\sim 5 \times 5 \mathrm{~mm}$ in area and $\sim 2 \mathrm{~mm}$ in height were glued onto Aluminium Specimen Stubs from Agar Scientific with Carbon adhesive discs from Agar Scientific. Vacuum was then applied and the samples were sputtered with a thin layer of gold. If the sputtering device was out of order, the samples were steamed with carbon instead. Sputtering with gold or steaming with carbon did not have significant influence on the quality of the SEM pictures later. A CamScan CS44 SEM from Tescan with secondary electron imaging (SEI) was used for the determination of the average pore diameter $d_{\text {pore, }}$ the shape-factor $S$ of the pore cross-sections, and the thickness/area of the 
pore wall and layers $t_{\text {pore wall }} / A_{\text {pore wall }}, t_{\text {outer layer }} / A_{\text {outer layer, }}$ and $t_{\text {inner layer }} / A_{\text {inner layer. To enable the }}$ determination of $d_{\text {pore }}$ and $S$, the circumference $C_{\text {pore }}$ and area $A_{\text {pore }}$ were determined with ImageJ. The outline of a pore cross-section was retraced manually which allowed ImageJ to calculate $C_{\text {pore }}$ and $A_{\text {pore. }} A_{\text {pore wall }}, A_{\text {outer layer, }}$ and $A_{\text {inner layer were determined analogously in one }}$

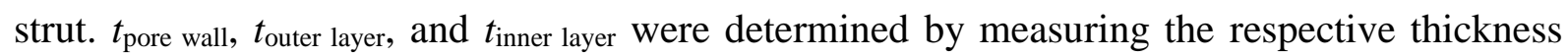
perpendicular to the direction the pore wall or layer is running along. For the analysis of the texture of the layers in the pore wall, a Zeiss GeminiSEM 500 SEM from Zeiss was used.

\subsubsection{Analysis of Mechanical Behavior}

The mechanical behavior of the macroporous PS/polyDVB samples as well as of the bulk samples used for normalization was examined with a universal testing machine. The samples were cut such that a cylindrical shape was obtained. Before cutting, the samples were cooled with liquid nitrogen. The main focus was put on cutting the sample such that the top and bottom of the cylinder were as parallel and as flat as possible. To calculate the density of the samples, the diameter and the height were measured with a caliper (accuracy $0.02 \mathrm{~mm}$ ). The diameter of the cylindrical samples was typically between $3.3-3.5 \mathrm{~mm}$ and their height was between $6-$ $8 \mathrm{~mm}$. The mass of the sample was determined with a high precision scale (accuracy $0.0001 \mathrm{~g}$ ). By dividing the density of the samples by the density of the bulk polymer, $\rho_{\text {rel }}$ was obtained (see equation (2.17)). Next, the samples were placed into the universal testing machine. A zwickiLine Z5.O (maximum capacity $5 \mathrm{kN}$ ) and the software testXpert III from ZwickRoell were used to record the stress-strain-curves. The compression rate was $1 \mathrm{~mm} \mathrm{~min}^{-1}$ and a Xforce HP load cell measured the stress. The Young's Modulus $E$ was determined from the slope of the linear elastic region of the stress-strain-curve. $F_{\max }$ was determined at the position where the linear elastic region goes over into the Plateau region.

Five samples were prepared for each composition. Of these five samples, one was used for the determination of the morphology with SEM, while the other four samples were used for the compression tests. This was done to connect a determined morphology to the recorded stressstrain-curves, i.e. to obtain structure-property-relationships. When a sample fractured during preparation, it could no longer be used in the compression tests, but was still viable to be examined with SEM. If more than one sample fractured, the compression tests were conducted with less than four samples. The reason why compression tests were conducted with more than one sample was to determine average values of $F_{\max }$ and $E_{\text {porous polymer. }}$ 


\subsection{Phase Diagram}

To investigate the response of the surfactant to polymerization, surfactant/styrene mixtures were prepared by weighing in the two components in a glass tube. A stirring bar was added to the mixture and the glass tube was placed over a magnetic stirrer and into a water bath. The temperature of the water bath was $70{ }^{\circ} \mathrm{C}$ to simulate the temperature of the polymerization. Small polystyrene (PS) pellets were added and dissolved stepwisely until the mixture changed from clear to turbid. This change indicated that the phase boundary had been crossed. The amount of added PS was determined by weighing the glass tube and subtracting the initial mass. Finally, the original surfactant/styrene mixture was added dropwisely until the mixture returned to the clear, one-phase region. After again weighing the glass tube, the phase boundary was determined more accurately.

\subsection{Interfacial Tension}

The interfacial tension $\gamma$ was measured with the Spinning Drop Video Tensiometer SVT 15 from DataPhysics Instruments. First, the capillary was filled bubble-free with $\mathrm{H}_{2} \mathrm{O}$. Next, $\sim 100 \mu \mathrm{L}$ of a surfactant/monomer mixture was injected into the capillary. By spinning the capillary, a droplet consisting of the surfactant/monomer mixture formed. $\beta_{\text {surfactant }}$ was varied between 5 $20 \mathrm{wt} . \%$ in steps of $5 \mathrm{wt} . \%$, the temperature was varied between $25-70{ }^{\circ} \mathrm{C}$ in steps of $15{ }^{\circ} \mathrm{C}$, and spinning rates were varied between $3000-13000 \mathrm{rpm}$ in steps of $2000 \mathrm{rpm}$. $\beta_{\text {styrene was }}$ kept constant at 50 wt.\%. LaPlace Young Fitting was used by the software SVT20_uEye to calculate $\gamma$ from the shape of the droplet. For LaPlace Young Fitting, the density of both $\mathrm{H}_{2} \mathrm{O}$ and the surfactant/monomer mixtures had to be known. For $\mathrm{H}_{2} \mathrm{O}$, the temperature-dependent values were already known by the software. For the surfactant/monomer mixtures, the Density Meter DMA 5000 A from Anton Paar was used to measure the temperature-dependent density.

\subsection{Small angle neutron scattering}

The small angle neutron scattering (SANS) spectra were recorded at the KWS-1 spectrometer at the Heinz Maier-Leibnitz Zentrum (MLZ) in Munich, Germany. A mixture of surfactant and deuterated styrene with $\beta_{\text {surfactant }}=10 \mathrm{wt}$. $\%$ was prepared and placed over $\mathrm{D}_{2} \mathrm{O}$ without allowing the two phases to mix and form an emulsion. After 8 minutes, 13.5 hours, and 1.5 days, a small amount of the upper monomer phase was removed from the sample, transferred into a cuvette, and placed into the beam path of the SANS device. The neutron wavelength was $4.93 \AA$ and 
the scattering intensity $q$ was varied between $0.00978-0.669 \AA^{-1}$. To enable this, collimation/detector distances of $8 \mathrm{~m} / 8 \mathrm{~m}$ and $8 \mathrm{~m} / 1.5 \mathrm{~m}$ were used. The normalization of the scattering density to an absolute scale was achieved by the incoherent scattering of reference materials. These were $\mathrm{H}_{2} \mathrm{O}$ and Perspex (trade name of poly(methyl methacrylate) (PMMA)). The evaluation software QtiKWS was used for raw data treatment, masking, and radial averaging. As a result, one-dimensional scattering curves were obtained. The fitting of the SANS spectrum was achieved with a Gaussian shell model [Fos08] where the addition of a form factor $P(q)$ and a structure factor $S(q)$ yield a simulated $I(q)$ spectrum.

\subsection{Freeze Fracture Electron Microscopy}

A mixture of surfactant, styrene, and DVB was prepared and placed over $\mathrm{H}_{2} \mathrm{O}$ without mixing the two phases, where $\beta_{\text {surfactant }}=10 \mathrm{wt} . \%$ and $\beta_{\text {styrene }}=50 \mathrm{wt}$. $\%$. After a small fraction of the monomer phase was removed, a replica of it was made by using the EM BAF060 freeze-fracture and etching system from Leica. For this purpose, the sample was placed into a sandwich-like structure consisting of two copper grids and two copper plates and consequently frozen in liquid ethane. Next, the sample was fractured and its surface was first shadowed at $45^{\circ}$ with a $\sim 2 \mathrm{~nm}$ thick platinum-carbon layer and secondly covered at $90^{\circ}$ with a $\sim 20 \mathrm{~nm}$ thick pure carbon layer. This process was conducted in the vacuum chamber of the BAFO6O and at a temperature of $-150{ }^{\circ} \mathrm{C}$. Finally, the replicas were washed with ethanol, dried, and examined at room temperature with the transmission electron microscope (TEM) EM10 from Zeiss.

\subsection{Dynamic Light Scattering}

As in Section 5.7, a mixture of surfactant, styrene, and DVB with $\beta_{\text {surfactant }}=10 \mathrm{wt} . \%$ and $\beta_{\text {styrene }}$ $=50 \mathrm{wt} . \%$ was prepared. The mixture was placed over $\mathrm{H}_{2} \mathrm{O}$ inside a capillary without mixing the two phases. The capillary was placed into the beam path of the dynamic light scattering (DLS) spectrometer 3DLS Spectrometer from LS Instruments such that the beam path crossed through the monomer phase. A helium/neon laser with a wavelength of $\lambda=561 \mathrm{~nm}$ was used and the scattering angle was kept constant at $90^{\circ}$. The scattering intensity was measured as count rate of photons with an avalanche photodiode (APD) detector. The software of the DLS spectrometer, $L S$ spectrometer, automatically calculated the hydrodynamic radius $r_{\mathrm{Hyd}}$ as well as its deviation from the slope of an intensity-time-correlation function $g_{2}-(\tau)$. Values were determined from $120 \mathrm{~s}$ time intervals for a total time span of $24 \mathrm{~h}$. 


\section{Appendix}

\subsection{Supplementary Calculations}

\section{(a) Relation between $\Phi_{\text {gas }}$ and $\rho_{\text {rel }} / P$}

In Section 2.3.2, it was stated that the relative density of a porous polymer $\rho_{\text {rel }}$ equals one minus the volume fraction of the gas $\Phi_{\text {gas }}$ (see equation (2.19)). Furthermore, it was stated that the porosity $P$ equals $\Phi_{\text {gas }}$ (see equation (2.20)). These relations are deduced in detail here. It holds

$$
\Phi_{\text {gas }}=\frac{V_{\text {gas }}}{V_{\text {gas }}+V_{\text {polymer }}}=\frac{V_{\text {gas }}}{V_{\text {total }}}=1-\frac{V_{\text {polymer }}}{V_{\text {total }}}
$$

with $V_{\text {gas }}$ being the volume of the gas, $V_{\text {polymer }}$ being the volume of the polymer, and $V_{\text {total }}$ being the combined volume of the gas and the polymer. The relative density $\rho_{\text {rel }}$ depends on the density of the porous polymer $\rho_{\text {porous polymer }}$ and the density of the bulk polymer $\rho_{\text {polymer }}$ In turn, $\rho_{\text {porous polymer }}$ and $\rho_{\text {polymer }}$ can be expressed by their respective masses $m_{\text {porous polymer }} / m_{\text {polymer }}$ and their respective volumes $V_{\text {porous polymer }} / V_{\text {polymer. It holds }}$

$$
\begin{gathered}
\rho_{\text {rel }}=\frac{\rho_{\text {porous polymer }}}{\rho_{\text {polymer }}}=\frac{m_{\text {porous polymer }} / V_{\text {porous polymer }}}{m_{\text {polymer }} / V_{\text {polymer }}} \\
=\frac{m_{\text {porous polymer }} \cdot V_{\text {polymer }}}{m_{\text {polymer }} \cdot V_{\text {porous polymer }}}=\frac{\left(m_{\text {gas }}+m_{\text {polymer }}\right) \cdot V_{\text {polymer }}}{m_{\text {polymer }} \cdot\left(V_{\text {gas }}+V_{\text {polymer }}\right)} .
\end{gathered}
$$

Next, the approximation is made that $m_{\text {gas }}$ is insignificant compared to $m_{\text {polymer }}$. It holds

$$
m_{\text {gas }}+m_{\text {polymer }} \cong m_{\text {polymer }}
$$

This enables a simplification of equation (6.2). It holds

$$
\rho_{\text {rel }} \cong \frac{m_{\text {polymer }} \cdot V_{\text {polymer }}}{m_{\text {polymer }} \cdot\left(V_{\text {polymer }}+V_{\text {gas }}\right)}=\frac{V_{\text {polymer }}}{V_{\text {polymer }}+V_{\text {gas }}}=\frac{V_{\text {polymer }}}{V_{\text {total }}} .
$$

Thus, the result of equation (6.4) can be inserted into equation (6.1). It holds

$$
\Phi_{\text {gas }}=1-\frac{V_{\text {polymer }}}{V_{\text {total }}}=1-\rho_{\text {rel }} .
$$


Finally, the relation between $\rho_{\text {rel }}$ and $P$ was already stated in equation (2.18). Thus, for the relation between $P$ and $\Phi_{\text {gas }}$, it holds

$$
P=1-\rho_{\text {rel }}=\Phi_{\text {gas }}
$$

\section{(b) Volume and Surface Area of a Rhombic Dodecahedron compared to a Sphere}

In Section 2.4, it was stated that for a constant volume, the surface area of a rhombic dodecahedron-shaped pore is roughly $10.5 \%$ larger than the surface area of a spherical droplet. In this paragraph, this value is calculated. For the surface area $A_{\text {sphere }}$ and volume $V_{\text {sphere }}$ of a sphere, it holds

$$
A_{\text {sphere }}=4 \pi r^{2}
$$

and

$$
V_{\text {sphere }}=\frac{4}{3} \pi r^{3}
$$

with $r$ being the radius of the sphere. For the surface area $A_{\text {rhombic dodecahedron }}$ and volume $V_{\text {rhombic }}$ dodecahedron of a rhombic dodecahedron, it holds

$$
A_{\text {rhombic dodecahedron }}=8 \cdot \sqrt{2} \cdot a^{2}
$$

and

$$
V_{\text {rhombic dodecahedron }}=\frac{16 \cdot \sqrt{3}}{9} a^{3}
$$

with $a$ being the edge length of the rhombic dodecahedron [www4, www5]. Approximately, the volume of a droplet $V_{\text {droplet }}$ is that of a sphere and the volume of a pore $V_{\text {pore }}$ is that of a rhombic dodecahedron. Assuming that the volume does not change during polymerization, it holds

$$
V_{\text {sphere }} \equiv V_{\text {droplet }}=V_{\text {pore }} \equiv V_{\text {rhombic dodecahedron }} \text {. }
$$

Thus, by rearranging equation (6.8) and equation (6.10), $a$ can be expressed with a coefficient and $r$. It holds 


$$
\begin{gathered}
\frac{16 \cdot \sqrt{3}}{9} a^{3}=\frac{4}{3} \pi r^{3} \\
a=\sqrt[3]{\frac{4 \pi \cdot 9}{3 \cdot 16 \cdot \sqrt{3}} r^{3}}=\sqrt[3]{\frac{\sqrt{3} \pi}{4} r} .
\end{gathered}
$$

Next, the result of equation (6.12) is inserted into equation (6.9). It holds

$$
\begin{gathered}
A_{\text {rhombic dodecahedron }}=8 \cdot \sqrt{2} \cdot a^{2}=8 \cdot \sqrt{2} \cdot\left(\sqrt[3]{\frac{\sqrt{3} \pi}{4} r}\right)^{2} \\
=8 \cdot \sqrt{2} \cdot \sqrt[3]{\frac{3 \pi^{2}}{16}} \cdot r^{2} .
\end{gathered}
$$

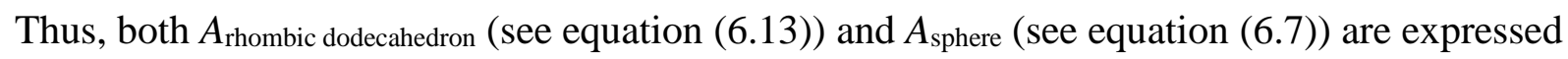
as a coefficient multiplied by $r^{2}$. Finally, their ratio shows how much larger $A_{\text {rhombic dodecahedron }}$ is compared to $A_{\text {sphere. It holds }}$

$$
\frac{A_{\text {rhombic dodecahedron }}}{A_{\text {sphere }}} \cong 1.105 \text {. }
$$

\section{(c) Conversion of mol\% to wt. $\%$}

In Section 3.1, an AIBN molar fraction $\chi_{\mathrm{AIBN}}=1.28 \mathrm{~mol} \%$ was converted into an AIBN mass fraction $\beta_{\mathrm{AIBN}}=1.82 \mathrm{wt} . \%$. Furthermore, a KPS molar fraction $\chi_{\mathrm{KPS}}=1.28 \mathrm{~mol} \%$ was converted into a KPS mass fraction $\beta_{\mathrm{KPS}}=2.98 \mathrm{wt}$. $\%$. In this paragraph, these conversions are calculated. It holds

$$
\begin{gathered}
\chi_{\mathrm{AIBN}}=1.28 \mathrm{~mol} \%=0.0128=\frac{n_{\mathrm{AIBN}}}{n_{\text {styrene }}+n_{\mathrm{DVB}}} \\
n_{\mathrm{AIBN}}=0.0128 \cdot\left(n_{\text {styrene }}+n_{\mathrm{DVB}}\right)
\end{gathered}
$$

with $n_{\mathrm{AIBN}}, n_{\text {styrene }}$ and $n_{\mathrm{DVB}}$ being the molar amounts of AIBN, styrene, and DVB, respectively. It holds

$$
\beta_{\mathrm{AIBN}}=\frac{m_{\mathrm{AIBN}}}{m_{\text {styrene }}+m_{\mathrm{DVB}}}
$$


with $m_{\mathrm{AIBN}}, m_{\text {styrene }}$ and $m_{\mathrm{DVB}}$ being the masses of AIBN, styrene, and DVB, respectively. In the samples by Quell et al. [Que15, Que16a, Que16b, Els17, Que17a, Que17b], the styrene/DVB mass ratio was kept constant at 50/50, Thus, it holds

$$
m_{\text {styrene }}=m_{\mathrm{DVB}}=m_{\text {monomer }} .
$$

Next, the results of equation (6.15) and equation (6.17) are inserted into equation (6.16). Additionally, the molar masses $M_{\mathrm{AIBN}}=164.21 \mathrm{~g} \mathrm{~mol}^{-1}, M_{\text {styrene }}=104.15 \mathrm{~g} \mathrm{~mol}^{-1}$, and $M_{\mathrm{DVB}}=$ $130.19 \mathrm{~g} \mathrm{~mol}^{-1}$ of AIBN, styrene, and DVB are used to convert the respective masses into molar amounts. It holds

$$
\begin{aligned}
\beta_{\mathrm{AIBN}} & =\frac{n_{\mathrm{AIBN}} \cdot M_{\mathrm{AIBN}}}{m_{\text {styrene }}+m_{\mathrm{DVB}}}=\frac{0.0128 \cdot\left(n_{\text {styrene }}+n_{\mathrm{DVB}}\right) \cdot M_{\mathrm{AIBN}}}{2 \cdot m_{\text {monomer }}} \\
= & \frac{0.0128 \cdot M_{\text {AIBN }}}{2} \cdot \frac{m_{\text {monomer }} / M_{\text {styrene }}+m_{\text {monomer }} / M_{\mathrm{DVB}}}{m_{\text {monomer }}} \\
= & 0.0064 \cdot M_{\mathrm{AIBN}} \cdot\left(\frac{1}{M_{\text {styrene }}}+\frac{1}{M_{\mathrm{DVB}}}\right)=0.0182 .
\end{aligned}
$$

For the conversion of $\chi_{\mathrm{KPS}}$ to $\beta_{\mathrm{KPS}}$, analogous calculations are conducted. It holds

$$
\begin{gathered}
\chi_{\mathrm{KPS}}=1.28 \mathrm{~mol} \%=0.0128=\frac{n_{\mathrm{KPS}}}{n_{\text {styrene }}+n_{\mathrm{DVB}}} \\
n_{\mathrm{KPS}}=0.0128 \cdot\left(n_{\text {styrene }}+n_{\mathrm{DVB}}\right)
\end{gathered}
$$

with $n_{\text {KPS }}$ being the molar amount of KPS. Since KPS is dissolved in $\mathrm{H}_{2} \mathrm{O}, \beta_{\mathrm{KPS}}$ is calculated with the KPS mass $m_{\mathrm{KPS}}$ and the $\mathrm{H}_{2} \mathrm{O}$ mass $m_{\mathrm{H} 2 \mathrm{O}}$. It holds

$$
\beta_{\mathrm{KPS}}=\frac{m_{\mathrm{KPS}}}{m_{\mathrm{H} 2 \mathrm{O}}}
$$

The statement of equation (6.17) applies here as well. Additionally, $m_{\mathrm{H} 2 \mathrm{O}}$ was equal to the combined mass of monomers in the experiments of Quell et al. [Que15, Que16a, Que16b, Els17, Que17a, Que17b]. Thus, it holds

$$
m_{\mathrm{H} 20}=m_{\text {styrene }}+m_{\mathrm{DVB}}=2 \cdot m_{\text {monomer }} .
$$


Finally, the results of equation (6.19) and equation (6.21) are inserted into equation (6.20). To convert masses into molar amounts, $M_{\text {styrene }}=104.15 \mathrm{~g} \mathrm{~mol}^{-1}$ and $M_{\mathrm{DVB}}=130.19 \mathrm{~g} \mathrm{~mol}^{-1}$ are used again. The KPS molar mass $M_{\mathrm{KPS}}=270.32 \mathrm{~g} \mathrm{~mol}^{-1}$. It holds

$$
\begin{gathered}
\beta_{\mathrm{KPS}}=\frac{n_{\mathrm{KPS}} \cdot M_{\mathrm{KPS}}}{m_{\mathrm{H} 2 \mathrm{O}}}=\frac{0.0128 \cdot\left(n_{\text {styrene }}+n_{\mathrm{DVB}}\right) \cdot M_{\mathrm{KPS}}}{2 \cdot m_{\text {monomer }}} \\
=\frac{0.0128 \cdot M_{\mathrm{KPS}}}{2} \cdot \frac{m_{\text {monomer }} / M_{\text {styrene }}+m_{\text {monomer }} / M_{\mathrm{DVB}}}{m_{\text {monomer }}} \\
=0.0064 \cdot M_{\mathrm{KPS}} \cdot\left(\frac{1}{M_{\text {styrene }}}+\frac{1}{M_{\mathrm{DVB}}}\right)=0.0298 .
\end{gathered}
$$

\section{(d) Different determinations of $d_{\text {pore }}$}

In Section 3.1.1, the diameter of the pores $d_{\text {pore }}$ was calculated as if the pore cross-sections were circular (see equation (3.1)). Here, this method is compared to the determination of $d_{\text {pore }}$ in a hexagon as the corner-to-corner distance, edge-to-edge distance, and the mean value of these two distances. Using the edge length $a$ of a hexagon, for the diameter corner-to-corner $d_{\text {corner-to- }}$ corner it holds

$$
d_{\text {corner-to-corner }}=2 a \text {. }
$$

For the diameter edge-to-edge, it holds

$$
d_{\text {edge-to-edge }}=\sqrt{3} a \approx 1.732 a \text {. }
$$

For the mean value of equation (6.23) and equation (6.24), it holds

$$
d_{\text {mean }}=\frac{2 a+\sqrt{3} a}{2}=\left(1+\frac{\sqrt{3}}{2}\right) a \approx 1.866 a
$$

For the calculation of the diameter as if the pore cross-sections were circular, the area of a hexagon $A_{\text {Hexagon }}$ is required. It holds

$$
A_{\text {Hexagon }}=\frac{3}{2} \cdot \sqrt{2} \cdot a^{2} .
$$

The result of equation (6.26) is now inserted into equation (3.1). It holds 


$$
\begin{aligned}
d_{\text {circular }} & =2 \cdot \sqrt{\frac{A_{\text {Hexagon }}}{\pi}}=2 \cdot \sqrt{\frac{3 / 2 \cdot \sqrt{2} \cdot a^{2}}{\pi}} \\
& =\sqrt{\frac{6 \cdot \sqrt{2}}{\pi}} \cdot a \approx 1.643 a
\end{aligned}
$$

Thus, when $d_{\text {pore }}$ is calculated as if the pore cross-section was circular, the obtained result is $17.85 \%$ (equation (6.23)) smaller than if it was calculated from the distance corner-to-corner. For the distance edge-to-edge or the mean value of the former two, the difference is $5.14 \%$ (equation (6.24)) or $11.95 \%$ (equation (6.25)), respectively.

\section{(e) Calculation of $\beta_{\text {surfactant at interface }}$}

In Section 3.2.1, it was stated that even for the lowest surfactant mass fraction $\beta_{\text {surfactant, }}$ the majority of the surfactant molecules are dissolved in the continuous phase and not located at the water/monomer interface. Here, the surfactant mass fraction necessary to cover the interface

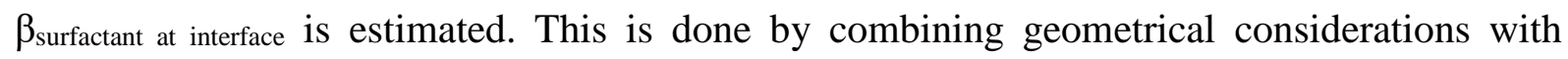
reasonable assumptions about the head group area $A_{\text {head group }}$ of the surfactant. From the radius $r_{\text {droplet }}$ of a water droplet inside a HIPE, its surface area

$$
A_{\text {droplet }}=4 \cdot \pi \cdot r_{\text {droplet }^{2}}
$$

and its volume

$$
V_{\text {droplet }}=\frac{4}{3} \cdot \pi \cdot r_{\text {droplet }}^{3}
$$

can be calculated. Since the droplets are monodiperse and reside in a HIPE, it is also possible to calculate the volume of the continuous monomer phase $V_{\text {continuous phase }}$ surrounding one droplet. It holds

$$
V_{\text {continuous phase }}=\frac{0.26}{0.74} \cdot V_{\text {droplet }} \text {. }
$$

Next, the molar amount of surfactant necessary to cover the interface of a droplet

$$
n_{\text {surfactant at interface }}=\frac{A_{\text {droplet }}}{A_{\text {head group }}} \cdot N_{\text {Avogadro }}{ }^{-1}
$$


is calculated from $A_{\text {droplet, }} A_{\text {head group, }}$ and the Avogadro number $N_{\text {Avogadro }}$. Here, reasonable

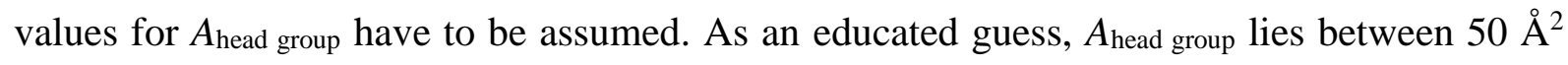
and $250 \AA^{2}$ [Wet01, Dom07]. $\beta_{\text {surfactant at interface can now be calculated with the help of equation }}$ (6.28), equation (6.29), equation (6.30), the density of the continuous phase $\rho_{\text {continuous phase, and }}$ the molar mass of the surfactant $M_{\text {Hypermer }}$ which is $833 \mathrm{~g} \mathrm{~mol}^{-1}$ [Gra13]. It holds

$$
\begin{gathered}
\beta_{\text {surfactant at interface }}=\frac{m_{\text {surfactant at interface }}}{m_{\text {continuous phase }}} \\
=\frac{n_{\text {surfactant at interface }} \cdot M_{\text {Hypermer }}}{V_{\text {continuous phase }} \cdot \rho_{\text {continuous phase }}} \\
=\frac{A_{\text {droplet }} \cdot M_{\text {Hypermer }}}{A_{\text {head group }} \cdot N_{\text {Avogadro }} \cdot V_{\text {continuous phase }} \cdot \rho_{\text {continuous phase }}} \\
=\frac{4 \cdot \pi \cdot r_{\text {droplet }}{ }^{2} \cdot M_{\text {Hypermer }}}{A_{\text {head group }} \cdot N_{\text {Avogadro }} \cdot 0.26 / 0.74 \cdot 4 / 3 \cdot \pi \cdot r_{\text {droplet }}{ }^{3} \cdot \rho_{\text {continuous phase }}} \\
=\frac{0.74 \cdot 3 \cdot M_{\text {Hypermer }}}{A_{\text {head group }} \cdot N_{\text {Avogadro }} \cdot 0.26 \cdot r_{\text {droplet }} \cdot \rho_{\text {continuous phase }}} .
\end{gathered}
$$

Since $M_{\text {Hypermer }}, N_{\text {Avogadro, }}$, and $\rho_{\text {continuous phase }}$ are all constant and known, $\beta_{\text {surfactant at interface }}$ only depends on $A_{\text {head group }}$ and $r_{\text {droplet. }}$ For example, for $r_{\text {droplet }}=40 \mu \mathrm{m}\left(d_{\text {droplet }}=80 \mu \mathrm{m}\right)$ and $A_{\text {head }}$ group $=50 \AA^{2}$ or $=250 \AA^{2}, \beta_{\text {surfactant at interface }}=0.065 \mathrm{wt} . \%$ or $=0.013 \mathrm{wt}$. $\%$. These values are

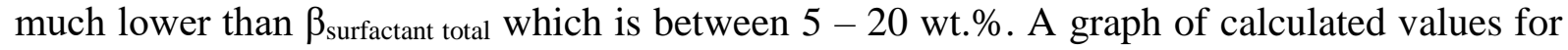

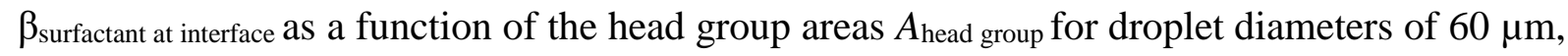
$80 \mu \mathrm{m}$, and $100 \mu \mathrm{m}$ is shown in Figure 6.5.

\subsection{Supplementary Figures}

\section{(a) Variation of $\beta_{\text {styrene between }} 50$ - 10 wt. \%}

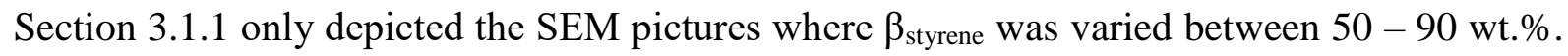
The SEM pictures of samples where $\beta_{\text {styrene }}=50 \mathrm{wt} . \%,=30 \mathrm{wt} . \%$, and $=10 \mathrm{wt} . \%$ are depicted in Figure 6.1. $\beta_{\mathrm{KPS}}$ is kept constant at $2.98 \mathrm{wt} . \%$ and $\beta_{\text {surfactant }}$ is kept constant at $10 \mathrm{wt} . \%$. The magnifications are 100-fold (left column), 500-fold (middle column), and 1000-fold (right column). In Figure 6.1, the 2D pore cross-sections fairly monodisperse and close-packed (see Figure 6.1 (left column)). Furthermore, the shape of the pore cross-sections is always hexagonal (see Figure 6.1 (middle column)) and the pore walls consist of three layers (see Figure 6.1 (right 

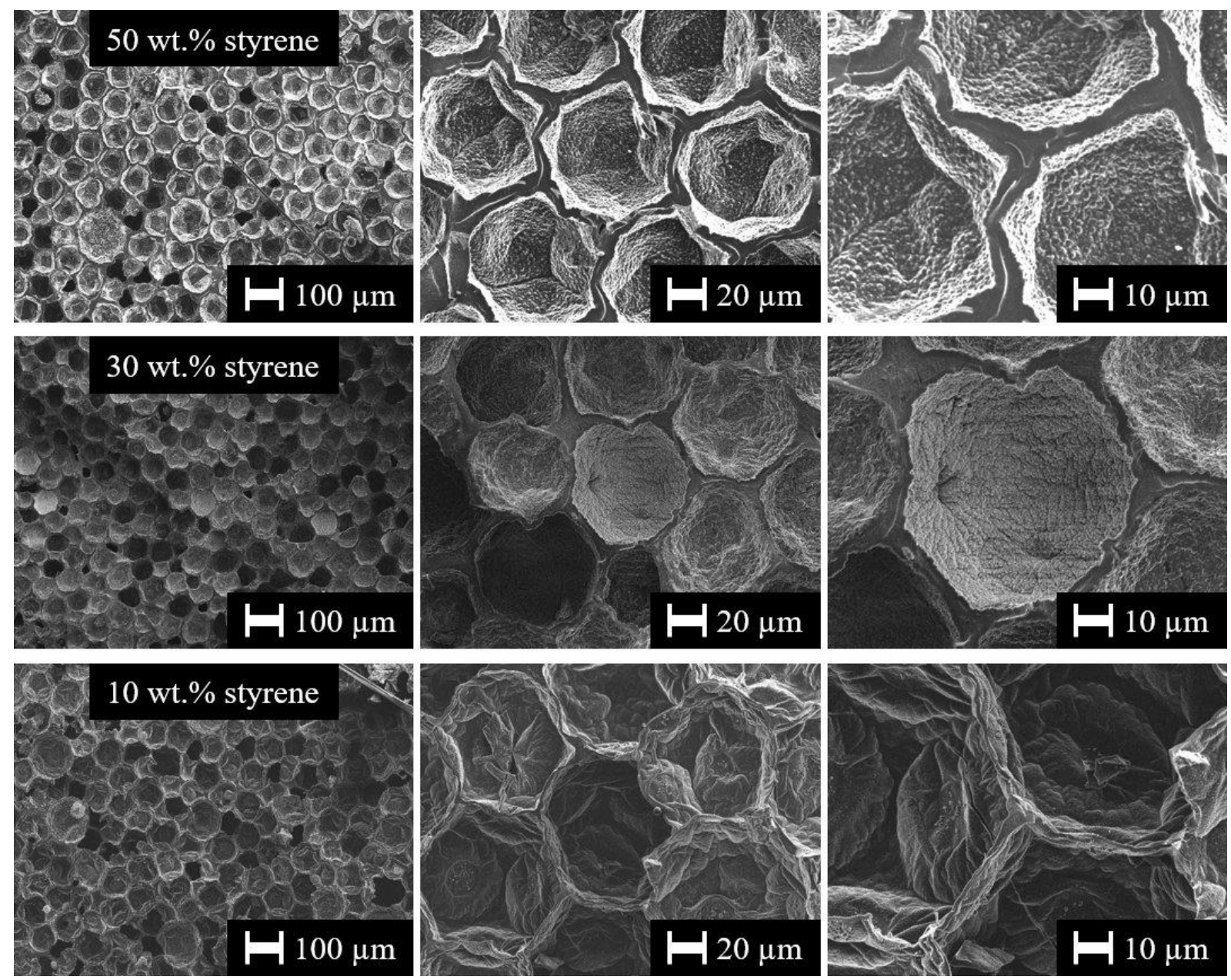

Figure 6.1: SEM pictures of macroporous PS/poly-DVB obtained via polymerization of monodisperse water-in-styrene/DVB HIPEs with $\beta_{\mathrm{KPS}}=2.98 \mathrm{wt} . \%, \beta_{\text {surfactant }}=10 \mathrm{wt} . \%$, and $\beta_{\text {styrene }}=50 \mathrm{wt} . \%,=30 \mathrm{wt} . \%$, and $=10 \mathrm{wt} . \%$.

column)). This means that the morphology does not change when $\beta_{\text {styrene }}$ is decreased from 50 wt.\% to $10 \mathrm{wt} . \%$, while the pore cross-sections became increasingly polyhedral and disordered

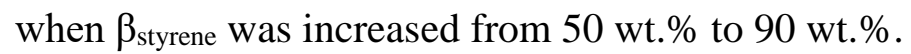

\section{(b) Determination of $C, A, t_{\text {pore wall, }} t_{\text {outer layer, and } t_{\text {inner layer }}}$}

To better illustrate how the circumference $C$, the area $A$, the pore wall thickness $t_{\text {pore wall, }}$ the

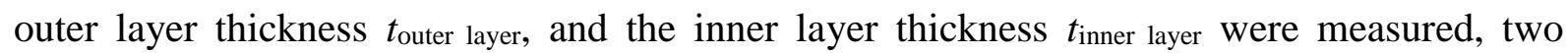
exemplary pictures are presented. Figure 6.2 (left) depicts how $C$ and $A$ were determined by manually encircling the pore cross-sections. From the encirclements, the image processing software Image $J$ calculated $C$ and $A$. $C$ and $A$ were determined from as many pore cross-sections

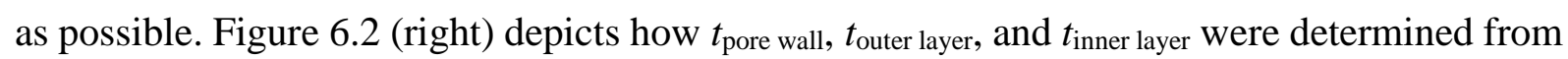
taking the respective thickness perpendicular to direction the pore wall / layer is running along. 


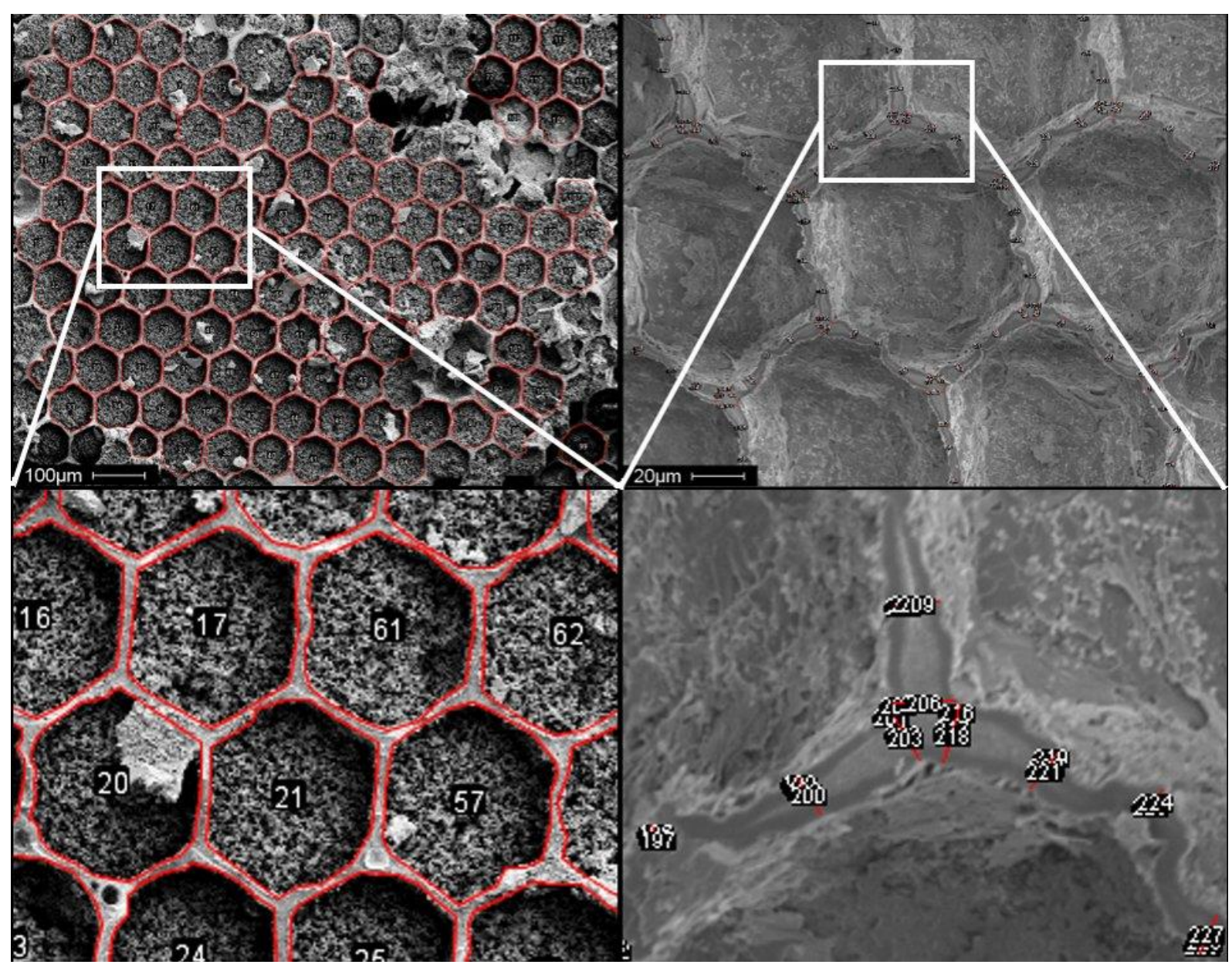

Figure 6.2: (left) Exemplary determination of the circumference $C$ and area $A$ of the pore crosssections and (right) exemplary determination of the pore wall thickness $t_{\text {pore wall, of the outer }}$

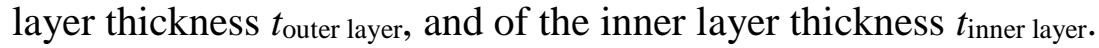

$t_{\text {pore wall, }}, t_{\text {outer layer, }}$ and $t_{\text {inner layer }}$ were determined close to the struts, in the films, and between these two. Furthermore, as many pore walls as possible were used to determine $t_{\text {pore wall }}, t_{\text {outer }}$ layer, and $t_{\text {inner layer. }}$

\section{(c) Variation of $\beta_{\mathrm{APS}}$ between $0.32-39.44$ wt. $\%$}

In Section 3.1.2, KPS was used as initiator. However, the maximum solubility of KPS in water is only $5 \mathrm{wt} . \%$, while that of APS is $62 \mathrm{wt} . \%$ [www2, www3]. To further examine if the shape of the pore cross-sections remains hexagonal if the initiator amount is raised to above $5 \mathrm{wt} . \%$, KPS was substituted by APS. The assumption was made that KPS and APS have roughly the same reactivity, though no experiments were conducted on that matter. In Figure 6.3, $\beta_{\text {APS }}$ is varied between $0.32-2.52$ wt. $\%$. In Figure 6.4, $\beta_{\text {APS }}$ is varied between $4.20-39.44$ wt.\%. In

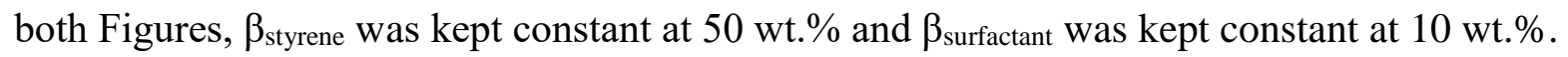



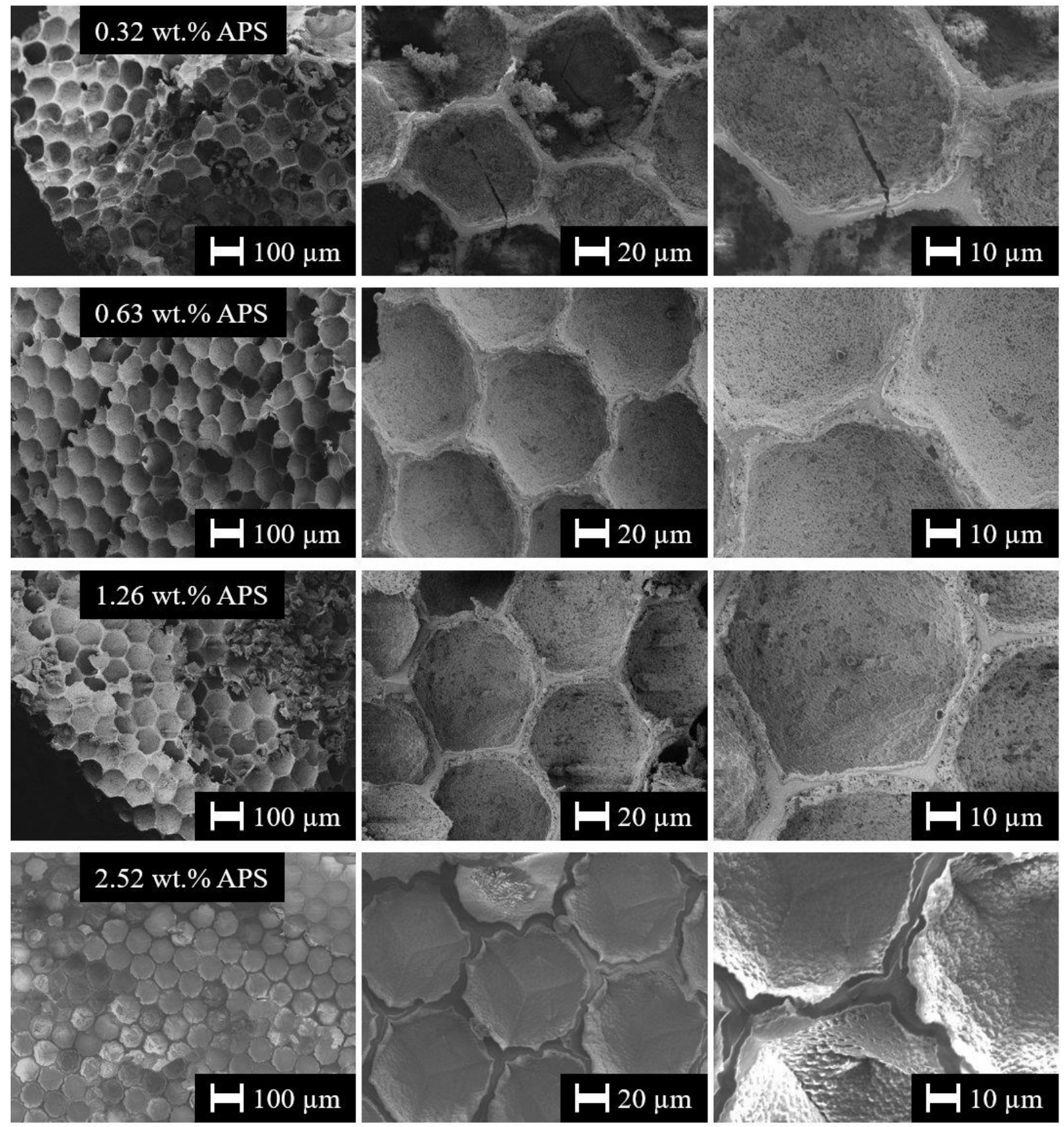

Figure 6.3: SEM pictures of macroporous PS/poly-DVB obtained via polymerization of monodisperse water-in-styrene/DVB HIPEs with $\beta_{\text {styrene }}=50 \mathrm{wt} . \%, \beta_{\text {surfactant }}=10 \mathrm{wt} . \%$, and $\beta_{\text {APS }}=0.32$ wt. $\%,=0.63$ wt. $\%,=1.26$ wt. $\%$, and $=2.52$ wt. $\%$.

Note that $\beta_{\mathrm{APS}}$ between $0.32-4.20 \mathrm{wt} \%$ is molarly equivalent to $\beta_{\mathrm{KPS}}$ between $0.37-4.96$ wt.\%. In contrast, $\beta_{\text {APS }}$ between 9.86 - 39.44 wt.\% has no counterpart with KPS. In Figure 6.3, the pores are always close-packed and fairly monodisperse. The shape of the pore cross-sections is hexagonal and the pore walls are comprised of three layers. Thus, these samples are very similar to their KPS counterparts in Figure 3.9. This implies that KPS and APS behave similar. In Figure 6.4, the pores become increasingly disordered and polydisperse when $\beta_{\mathrm{APS}}$ is increased. For $\beta_{\mathrm{APS}}=4.20 \mathrm{wt} . \%$ and $=9.86 \mathrm{wt} . \%$, the shape of the pore cross-sections is still 

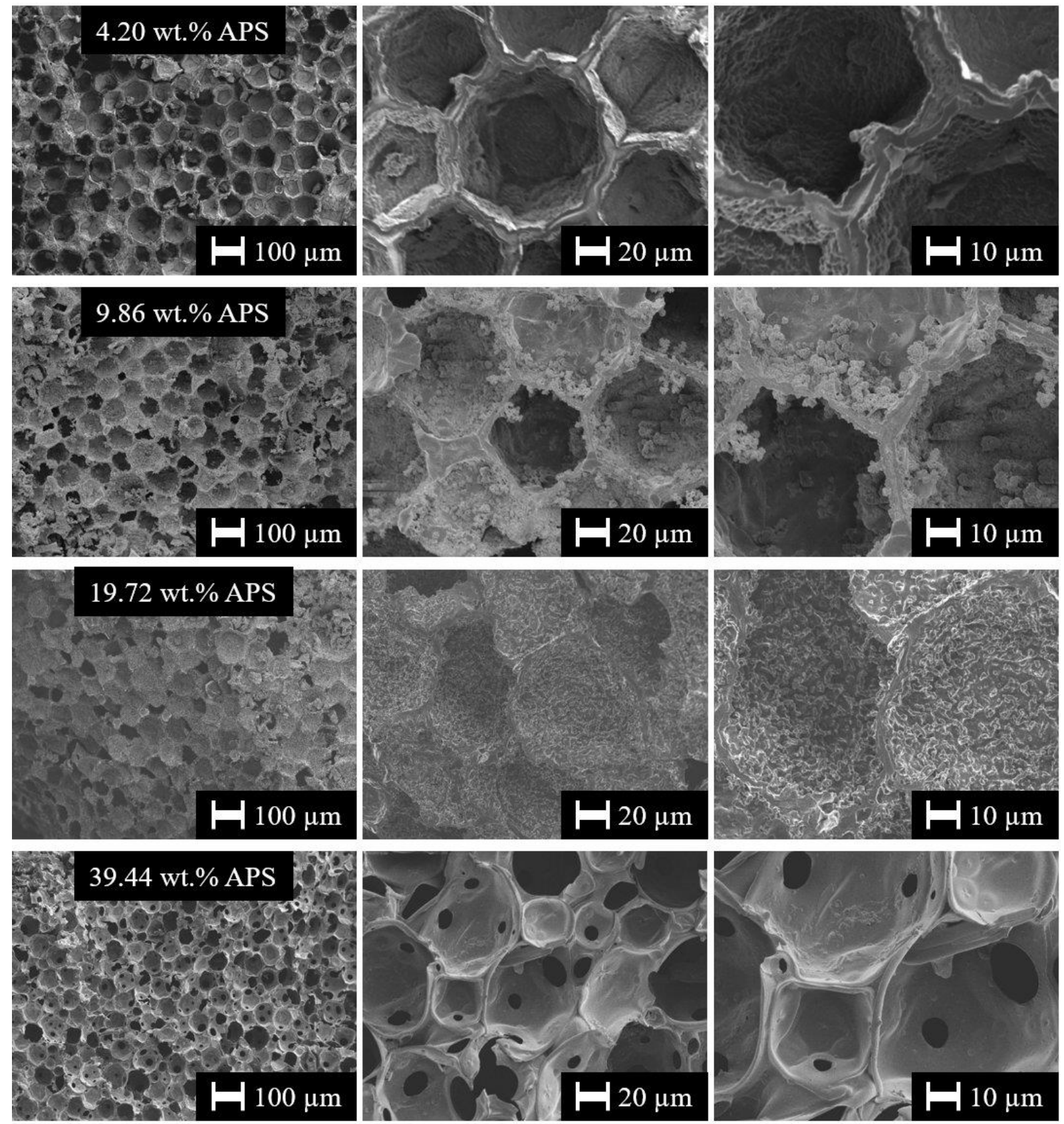

Figure 6.4: SEM pictures of macroporous PS/poly-DVB obtained via polymerization of monodisperse water-in-styrene/DVB HIPEs with $\beta_{\text {styrene }}=50 \mathrm{wt} . \%, \beta_{\text {surfactant }}=10 \mathrm{wt} . \%$, and $\beta_{\mathrm{APS}}=4.20 \mathrm{wt} . \%,=9.86 \mathrm{wt} . \%,=19.72 \mathrm{wt} . \%$, and $=39.44 \mathrm{wt} . \%$.

more or less hexagonal and three layers are observed in the pore walls. For $\beta_{\mathrm{APS}}=19.72 \mathrm{wt}$. $\%$, the pore cross-sections have no regular shape anymore and no layers in the pore walls can be found. For $\beta_{\mathrm{APS}}=39.44 \mathrm{wt} . \%$, the pores are polydisperse and the interconnectivity changed from closed-pore to open-pore. Overall, the SEM pictures in Figure 6.3 and Figure 6.4 show that even when $\beta_{\mathrm{APS}}$ is increased to absurd levels, the pore cross-sections do not become circular. Thus, these results also conflict with the idea of an osmotic transport of DVB as the reason for the transformation from spherical droplets to polyhedral pores. 


\section{(d) Calculation of $\beta_{\text {surfactant at interface }}$}

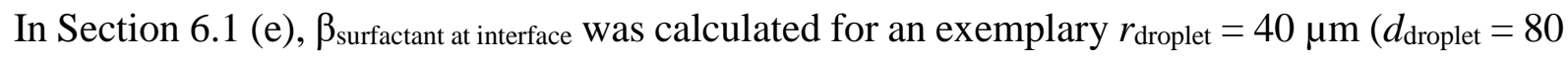
$\mu \mathrm{m})$ and for two exemplary $A_{\text {head group area }}=50 \AA^{2}$ and $=250 \AA^{2} . M_{\text {Hypermer }}, N_{\text {Avogadro, }}$, and $\rho_{\text {continuous }}$ phase were constant and known (see equation (6.32)). For a better overview, in Figure $6.5 \beta_{\text {surfactant }}$ at interface is calculated as function of $A_{\text {head group area }}$. The chosen droplet diameters are $d_{\text {droplet }}=60$ $\mu \mathrm{m}$ (dashed line) $=80 \mu \mathrm{m}$ (continuous line), and $=100 \mu \mathrm{m}$ (dotted line). These droplet diameters are in the range of the droplet diameter generated in this PhD thesis. $A_{\text {head group area }}$ was chosen between 10 - $300 \AA^{2}$ which ought to include the actual, but unknown $A_{\text {head group area }}$ of the surfactant Hypermer 2296 that was used in this $\mathrm{PhD}$ thesis. Figure 6.5 shows that even for the smallest $A_{\text {head group area }}$ and smallest $d_{\text {droplet}}, \beta_{\text {surfactant at interface }}$ is still much lower than the surfactant mass fractions that were used in this $\mathrm{PhD}$ thesis.

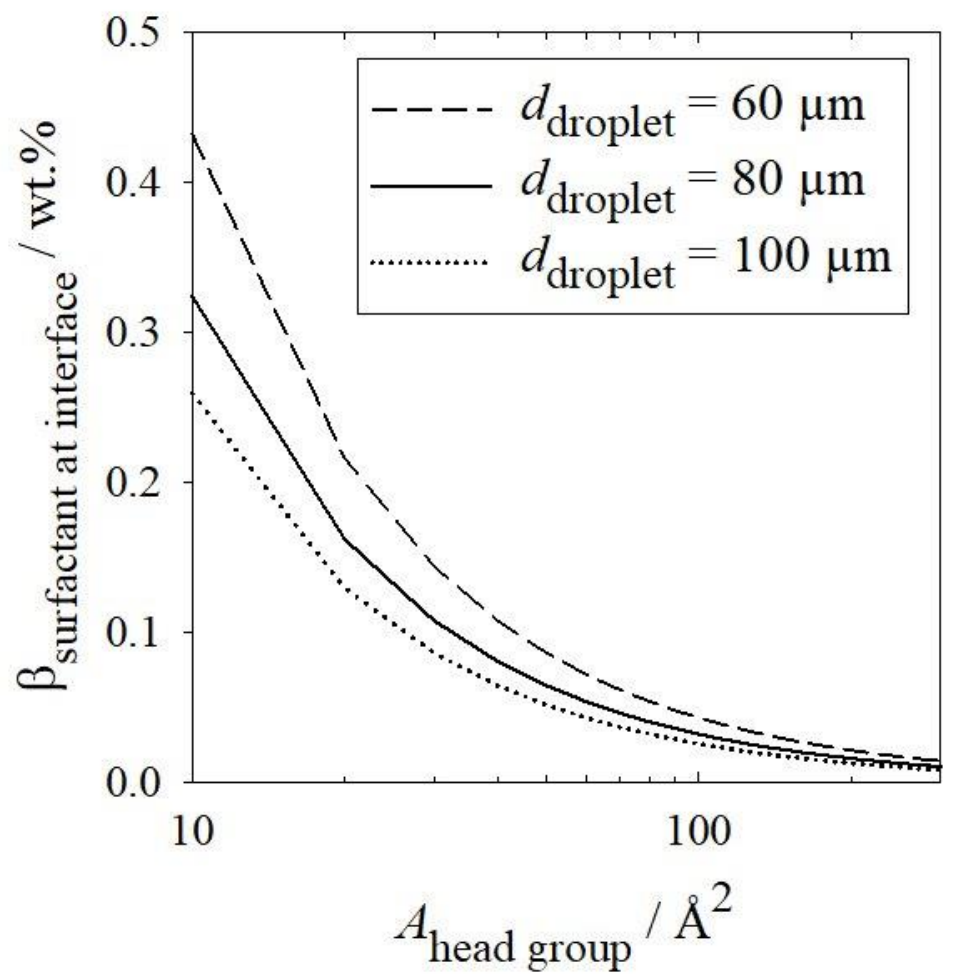

Figure 6.5: Calculated surfactant mass fraction $\beta_{\text {surfactant at interface }}$ necessary to cover the interfacial area of a water droplet with diameters of $60 \mu \mathrm{m}, 80 \mu \mathrm{m}$, and $100 \mu \mathrm{m}$ inside a HIPE as function of the head group area $A_{\text {head group area }}$ of the surfactant. 


\section{References}

[And18] Andrieux, S.; Drenckhan, W.; Stubenrauch, C.; Generation of Solid Foams with Controlled Polydispersity Using Microfluidics, Langmuir 2018, 34(4), 15811590.

[Ann02] Anna, S. L.; Bontoux, N.; Stone, H. A.; Formation of dispersions using „flow focusing“ in microchannels, Applied Physics Letters 2002, 82(3), 364-366.

[Ann16] Anna, S. L.; Droplets and Bubbles in Microfluidic Devices, Annual Review of Fluid Mechanics 2016, 48, 285-309.

[Bae09] Baekeland, L. H.; US942699 (A) - method of making insoluble products of phenol and formaldehyde, 1909.

[Ban11] Bandarian, M.; Shojaeia, A.; Rashidi, A. M.; Thermal, mechanical and acoustic damping properties of flexible open-cell polyurethane/multi-walled carbon nanotube foams: effect of surface functionality of nanotubes, Polymer International 2011, 60, 475-482.

[Bar62] Bartl, H; von Bonin, W.; Über die Polymerisation in umgekehrter Emulsion, Macromolecular Chemistry and Physics 1962, 57, 74-95.

[Bar85] Barby, D.; Haq, Z.; EP 0060138-Low Density Porous Cross-Linked Polymeric Materials and Their Preparation, 1985.

[Bar00] Barbetta, A.; Cameron, N. R.; Cooper, S. J.; High internal phase emulsions (HIPEs) containing divinylbenzene and 4-vinylbenzyl chloride and the morphology of the resulting PolyHIPE materials, Chemical Communications 2000, 0, 221-222.

[Bar10] Baroud, C. N.; Gallaire, F.; Dangla, R.; Dynamics of microfluidic droplets, Lab on a Chip 2010, 10, 2032-2045.

[Bee02] Beebe, D. J.; Mensing, G. A.; Walker, G. M.; Physics and Applications of Microfluidics in Biology, Annual Review of Biomedical Engineering 2002, 4, 261-286.

[Ber60] Bernal, J. D.; Mason, J.; Packing of Spheres: Co-ordination of Randomly Packed Spheres, Nature 1960, 188, 910-911. 
[Bin98] Binks, B. P.; Modern Aspects of Emulsion Science; The Royal Society of Chemistry, Cambridge, 1998.

[Bur76] Burwell, R. L. Jr.; Manual of Symbols and Terminology for Physiochemical Quantities and Units, Appendix III: Definitions, Terminology and Symbols in Colloid and Surface Chemistry - Part II: Heterogeneous Catalysis, Pure and Applied Chemistry 1976, 46(1), 71-90. (page 79)

[Cam96] Cameron, N. R.; Sherrington, D. C.; Albiston, L.; Gregory, D. P.; Study of the formation of the open-cellular morphology of poly(styrene/divinylbenzene) polyHIPE materials by cryo-SEM, Colloid \& Polymer Science 1996, 274(6), $592-595$.

[Cam97] Cameron, N. R.; Sherrington, D. C.; Preparation and glass transition temperatures of elastomeric PolyHIPE materials, Journal of Material Chemistry 1997, 7(11), 2209-2212.

[Cam00] Cameron, N. R.; Barbetta, A.; The influence of porogen type on the porosity, surface area and morphology of poly(divinylbenzene) PolyHIPE foams, Journal of Materials Chemistry 2000, 10, 2466-2471.

[Cam05] Cameron, N. R.; High internal phase emulsion templating as a route to welldefined porous polymers, Polymer 2005, 46, 1439-1449.

[Cho09] Choi, S.-W.; Cheong, I. W.; Kim, J.-H.; Xia, Y.; Preparation of Uniform Microspheres Using a Simple Device and Their Crystallization into ClosePacked Lattices, Small 2009, 5(4), 454-459.

[Chr07] Christopher, G. F.; Anna, S. L.; Microfluidic methods for generating continuous droplet streams, Journal of Physics D: Applied Physics 2007, 40, R319-R336.

[Chu09] Chung, K.-Y.; Mishra, N. C.; Wang, C.-C.; Lin, F.-H.; Lin, K.-H.; Fabricating scaffolds by microfluidics, Biomicrofluidics 2009, 3, 022403-(1-8).

[Cla16] Clayton, K. N.; Salameh, J. W.; Wereley, S. T.; Kinzer-Ursem, T. L.; Physical characterization of nanoparticle size and surface modification using particle scattering diffusometry; Biomicrofluidics 2016, 10 (5), 054107-(1-14).

[Cos14] Costantini, M.; Colosi, C.; Guzowski, J.; Barbetta, A.; Jaroszewicz, J.; Swieszkowski, W.; Dentinia, M.; Garstecki, P.; Highly ordered and tunable 
polyHIPEs by using microfluidics, Journal of Material Chemistry B 2014, 2, 2290-2300.

[Dab20] Dabrowski, M.; Jenkins, D; Cosgriff-Hernandez, E.; Stubenrauch, C.; Methacrylate-based polymer foams with controllable connectivity, pore shape, pore size and polydispersity, Physical Chemistry Chemical Physics 2020, 22, 155-168.

[Dom07] Dominguez, H.; Self-Aggregation of the SDS Surfactant at a Solid-Liquid Interface, Journal of Physical Chemistry B 2007, 111, 4054-4059.

[Dre15] Drenckhan, W.; Hutzler, S.; Structure and energy of liquid foams, Advances in Colloid and Interface Science 2015, 224, 1-15.

[Dus67] Dusek, K.; Phase Separation During the Formation of Three-Dimensional Polymers, Journal of Polymer Science: Part C 1967, 16, 1289-1299.

[Egi08] Egidi, E.; Gasparini, G.; Holdich, R.G.; Vladisavljević, G.T.; Kosvintsev, S.R.; Membrane emulsification using membranes of regular pore spacing: Droplet size and uniformity in the presence of surface shear, Journal of Membrane Science 2008, 323, 414-420.

[Els17] Elsing, J.; Quell, A.; Stubenrauch, C.; Toward Functionally Graded Polymer Foams Using Microfluidics, Advanced Engineering Materials 2017, 19, 1700195 .

[Eva99] Evans, D. F.; Wernerström, H.; The Colloidal Domain - Where Physics, Chemistry, Biology, and Technology meet; Wiley-VCH, New York, 1999.

[Eve72] Everett, D. H.; Manual of Symbols and Terminology for Physicochemical Quantities and Units, Appendix II: Definitions, Terminology and Symbols in Colloid and Surface Chemistry - Part I, Pure and Applied Chemistry 1972, 31(4), 579-638. (page 585)

[Fos08] Foster, T.; Sottmann, T.; Schweins, R.; Strey, R.; Small-angle neutron scattering from giantwater-in-oil microemulsion droplets. I. Ternary system, The Journal of Chemical Physics 2008, 128, 054502-(1-13).

[Gan01] Gañán-Calvo, A. M.; Gordillo, J. M.; Perfectly Monodisperse Microbubbling by Capillary Flow Focusing, Physical Review Letters 2001, 87(27), 274501-(1-4). 
[Gar05] Garstecki, P.; Gañán-Calvo, A. M, Whitesides, G. M.; Formation of bubbles and droplets in microfluidic systems, Bulletin of the Polish Academy of Sciences: Technical Sciences 2005, 53(4), 361-372.

[Gas08] Gasparini, G.; Kosvintsev, S.; Stillwell, M.; Holdich, R.; Preparation and characterization of PLGA particles for subcutaneous controlled drug release by membrane emulsification, Colloids and Surfaces B: Biointerfaces 2008, 61, 199207.

[Gib97] Gibson, L. J.; Ashby, M. F.; Cellular solids - Structure and properties, Cambridge University Press, Cambrigde, $2^{\text {nd }}$ edition, 1997.

[Giu17] Giustiniani, A.; Drenckhan, W.; Poulard, C.; Interfacial tension of reactive, liquid interfaces and its consequences, Advances in Colloid and Interface Science 2017, 247, 185-197.

[Gra93] Gravesen P.; Branebjerg, J.; Jensen, O. S.; Microfluidics - a review, Journal of Micromechanics and Microengineering 1993, 3, 168-182.

[Gra13] Graeber, N.; A Study of Fundamentals in Emulsion Templating for the Preparation of Macroporous Polymer Foams, Imperial College London, $\mathrm{PhD}$ Thesis, 2013 (p. 85).

[Hai91] Hainey, P.; Huxham, I. M.; Rowatt, B.; Sherrington, D. C.; Tetley, L.; Synthesis and Ultrastructural Studies of Styrene-Divinylbenzene Polyhipe Polymers, Macromolecules 1991, 24, 117-121.

[Hay83] Hayashi, T.; Ito, J.; Mitani, K.; Mizutani, Y.; Dynamic Mechanical Properties and Morphology of Styrene-Divinylbenzene Copolymer/Poly(vinyl Chloride) Systems, Journal of Applied Polymer Science 1983, 28, 2867-2880.

[Hal06] Hales, T. C.; Ferguson, S. P.; A Formulation of the Kepler Conjecture, Discrete \& Computational Geometry 2006, 36 (1), 21-69.

[Hil85a] Hild, G.; Okasha, R.; Kinetic investigation of the free radical crosslinking copolymerization in the pre-gel state, 1 , Styrene/m- and $p$-divinylbenzene systems, Makromolekulare Chemie 1985, 186, 93-110. 
[Hil85b] Hild, G.; Okasha, R.; Rempp, P.; Free radical crosslinking copolymerization in the post-gel state, 3, Swelling and mechanical properties of polystyrene networks, Makromolekulare Chemie 1985, 186, 407-422.

[Hil04] Hilgenfeldt, S.; Kraynik, A. M.; Reinelt, D. A.; Sullivan, J. M.; The structure of foam cells: Isotropic Plateau polyhedra, Europhysics Letters 2004, 67(3), 484490.

[Hol10] Holdich, R.G.; Dragosavac, M.M.; Vladisavljević G.T.; Kosvintsev, S.R.; Membrane Emulsification with Oscillating and Stationary Membranes, Industrial \& Engineering Chemical Research 2010, 49, 3810-3817.

[Jia99] Jiao, J.; Kramer, E. J.; de Vos, S.; Möller, M.; Koning, C.; Polymer interface instability caused by grafting reaction, Polymer 1999, 40, 3585-3588.

[Jon06] de Jong, J.; Lammertink, R. G. H.; Wessling, M.; Membranes and microfluidics: a review, Lab on a Chip 2006, 6, 1125-1139.

[Kim11] Kimmins, S. D.; Cameron, N. R.; Functional Porous Polymers by Emulsion Templating: Recent Advances, Advanced Functional Materials 2011, 21, 211225.

[Koc20] Koch, L.; Drenckhan, W.; Stubenrauch, C.; Porous polymers via emulsion templating: pore deformationduring solidification cannot be explained by an osmotic transport!, Colloid and Polymer Science 2020.

[Koc21] Koch, L.; Botsch, S.; Stubenrauch, C.; Emulsion templating: Unexpected morphology of monodisperse macroporous polymers, Journal of Colloid and Interface Science 2021, 582(B), 834-841.

[Kos05] Kosvintsev, S.R.; Gasparini, G.; Holdich, R.G.; Cumming, I.W.; Stillwell, M.T.; Liquid-Liquid Membrane Dispersion in a Stirred Cell with and without Controlled Shear, Industrial \& Engineering Chemical Research 2005, 44, 9323 9330.

[Kra04] Kraynik, A. M.; Reinelt, D. A.; van Swol, F.; Structure of Random Foam, Physical Review Letters 2004, 93(20), 208301-(1-4). 
[Kra05] Kraynik, A. M.; Reinelt, D. A.; van Swol, F.; Structure of random bidisperse foam, Colloids and Surfaces A: Physicochemical and Engineering Aspects 2005, $263,11-17$.

[Kra06] Kraynik, A. M.; The Structure of Random Foam, Advanced Engineering Materials 2006, 8(9), 900-906.

[Kun68] Kun, K. A.; Kunin, R.; Macroreticular Resins. III. Formation of Macroreticular Styrene-Divinylbenzene Copolymers, Journal of Polymer Science: Part A-1 1968, 6, 2689-2701.

[Lan95] Landrock, A. H.; Handbook of Plastic Foams - Types, Properties, Manufacture and Applications, Noyes Publications, Park Ridge, 1995.

[Lec10] Lechner, M. D.; Gehrke, K.; Nordmeier, E. H.; Makromolekulare Chemie - Ein Lehrbuch für Chemiker, Physiker, Materialwissenschaftler und Verfahrenstechniker; Birkhäuser Verlag, Basel - Boston- Berlin, $4^{\text {th }}$ edition, 2010.

[Lee07] Lee, S.-T.; Park, C. B.; Ramesh, N. S.; Polymeric foams, Taylor \& Francis Group, Boca Raton, 2007.

[Lin11] Lin, J.-Y.; Lin, W.-J.; Hong, W.-H.; Hung, W.-C.; Nowotarski, S. H.; Montenegro Gouveai, S.; Cristo, I.; Lin, K.-H.; Morphology and organization of tissue cells in 3D microenvironment of monodisperse foam scaffolds, Soft Matter 2011, 7, 10010-10016.

[Lyk05] Lykema, J.; Fundamentals of Interface and Colloid Science - Volume V: Soft Colloids; Elsevier, Amsterdam, 2005.

[Mae13] Maestro, A.; Drenckhan, W.; Rio, E.; Höhler, R.; Liquid dispersions under gravity: volume fraction profile and osmotic pressure, Soft Matter 2013, 9, 25312540.

[Man92] Manz, A.; Harrison, D. J.; Verpoorte, E. M. J.; Fettinger, J. C.; Paulus, A.; Lüdi, H.; Widmer, H. M.; Planar chips technology for miniaturization and integration of separation techniques into monitoring systems - Capillary electrophoresis on a chip, Journal of Chromatography 1992, 593, 253-258. 
[Mil07] Mills, N.; Polymer Foams Handbook: Engineering and Biomechanics Applications and Design Guide, Elsevier, Amsterdam, 2007.

[Nis12] Nisisako, T.; Ando, T.; Hatsuzawa, T.; High-volume production of single and compound emulsions in a microfluidic parallelization arrangement coupled with coaxial annular world-to-chip interfaces, Lab on a chip 2012, 12, 3426-3435.

[Ost00] Ostwald, W.; Über die vermeintliche Isomerie des roten und gelben Quecksilberoxyds und die Oberflächenspannung fester Körper, Zeitschrift für Physikalische Chemie 1900, 34 (1), 495-503.

[Pat12] Patashinski, A. Z.; Orlik, R.; Paclawski, K.; Ratner, M. A.; Grzybowski, B. A.; The unstable and expanding interface between reacting liquids: theoretical interpretation of negative surface tension, Soft Matter 2012, 8, 1601-1608.

[Que15] Quell, A.; Elsing, J.; Drenckhan, W.; Stubenrauch, C.; Monodisperse Polystyrene Foams via Microfluidics - A Novel Templating Route, Advanced Engineering Materials 2015, 17, 604-609.

[Que16a] Quell, A.; de Bergolis, B.; Drenckhan, W.; Stubenrauch, C.; How the Locus of Initiation Influences the Morphology and the Pore Connectivity of a Monodisperse Polymer Foam, Macromolecules 2016, 49, 5059-5067.

[Que16b] Quell, A.; Monodisperse Emulsions as Template for Highly Structured Polymer Foams, University of Stuttgart, PhD Thesis, 2016.

[Que17a] Quell, A.; Heitkam, S.; Drenckhan, W.; Stubenrauch, C.; Creating Honeycomb Structures in Porous Polymers by Osmotic Transport, ChemPhysChem 2017, 18, 451-454.

[Que17b] Quell, A.; Sottmann, T.; Stubenrauch, C.; Diving into the Finestructure of Macroporous Polymer Foams Synthesized via Emulsion Templating: A Phase Diagram Study, Langmuir 2017, 33, 537-542.

[Rin71] Rinde, J. A.; Hoge, K. G.; Time and Temperature Dependence of the Mechanical Properties of Polystyrene Bead Foam, Journal of Applied Polymer Science 1971, $15,1377-1395$.

[Ros12] Rosen, M. J.; Kunjappu, J. T.; Surfactants and Interfacial Phenomena, John Wiley \& Sons, 4th edition, Hoboken, 2012. 
[Sch75] Schwachula, G.; Calculation of the copolymerization parameters in the ternary copolymerization system of styrene $/ m$-divinyl-benzene $/ p$-divinylbenzene, Journal of Polymer Science: Polymer Symposia 1975, 53, 107-112.

[Sch05] Schramm, L. L.; Emulsions, Foams, and Suspensions - Fundamentals and Applications, Wiley-VCH, Weinheim, 2005.

[Sil05] Silverstein, M. S.; Tai, H.; Sergienko, A.; Lumelsky, Y.; Pavlovsky, S.; PolyHIPE: IPNs, hybrids, nanoscale porosity, silica monoliths and ICP-based sensors, Polymer 2005, 46, 6682-6694.

[Sil14] Silverstein, M. S.; PolyHIPEs: Recent advances in emulsion-templated porous polymers, Progress in Polymer Science 2014, 39, 199-234.

[Sop72] Soper, B.; Haward, R. N.; White, E. F. T.; Intramolecular Cyclization of Styrene-p-Divinylbenzene Copolymers, Journal of Polymer Science: Part A-1 1972, 10, 2545-2464.

[Squ05] Squires, T. M.; Quake, S. R.; Microfluidics: Fluid physics at the nanoliter scale, Reviews of Modern Physics 2005, 77, 977-1025.

[Sto65] Storey, B. T.; Copolymerization of Styrene and $p$-Divinylbenzene. Initial Rates and Gel Points, Journal of Polymer Science: Part A 1965, 3, 265-282.

[Stu09] Stubenrauch, C.; Microemulsions - Background, New Concepts, Applications, Perspectives; Wiley-VCH, Oxford, 2009.

[Su13] Su, W.-F.; Principles of Polymer Design and Synthesis, Springer, Berlin Heidelberg, 2013.

[Sun93] Sundell, M. J.; Pajunen, E. O.; Hormi, O. O. E.; Näsman, J. H.; Crosslinked Polystyrene with Improved Mechanical Properties, Journal of Polymer Science: Part A: Polymer Chemistry 1993, 31, 2305-2311.

[Sun12] Sun, Y.-S.; Peng, S.-W.; Lin, K.-H.; Cheng, J.-Y.; Electrotaxis of lung cancer cells in ordered three-dimensional scaffolds, Biomicrofluidics 2012, 6, 014102 $(1-14)$.

[Tab05] Tabeling, P.; Introduction to Microfluidics, Oxford University Press, Oxford 2005. 
[Tai01a] Tai, H.; Sergienko, A.; Silverstein, M. S.; Organic-inorganic networks in foams from high internal phase emulsion polymerizations, Polymer 2001, 42, 44734482.

[Tai01b] Tai, H.; Sergienko, A.; Silverstein, M. S.; High Internal Phase Emulsion Foams: Copolymers and Interpenetrating Polymer Networks, Polymer Engineering and Science 2001, 41(9), 1540-1552.

[Ta109] Talha Gokmen, M.; Van Camp, W.; Colver, P. J.; Bon, S. A. F.; Du Prez, F. E.; Fabrication of Porous "Clickable" Polymer Beads and Rods through Generation of High Internal Phase Emulsion (HIPE) Droplets in a Simple Microfluidic Device, Macromolecules 2009, 42, 9289-9294.

[Teh08] Teh, S.-Y.; Lin, R.; Hung, L.-H.; Lee, A. P.; Droplet microfluidics, Lab on a Chip 2008, 8, 198-220.

[Tho01] Thorsen, T.; Roberts, R. W.; Arnold, F. H.; Quake, S. R.; Dynamic Pattern Formation in a Vesicle-Generating Microfluidic Device, Physical Review Letters 2001, 86(18), 4163-4166.

[Tie14] Tieke, B.; Makromolekulare Chemie - Eine Einführung, Wiley-VCH, Weinheim, $3^{\text {rd }}$ edition, 2014.

[Umb00] Umbanhowar, P. B.; Prasad, V.; Weitz, D. A.; Monodisperse Emulsion Generation via Drop Break Off in a Coflowing Stream, Langmuir 2000, 16, 347351.

[Vit03] Vitale, S. A.; Katz, J. L.; Liquid Droplet Dispersions Formed by Homogeneous Liquid-Liquid Nucleation: The Ouzo Effect; Langmuir 2003, 19 (10), 41054110.

[Wan11] Wang, C-C.; Yang, K.-C.; Lin, K.-H.; Liu, H.-C.: Lin, F.-H.; A highly organized three-dimensional alginate scaffold for cartilage tissue engineering prepared by microfluidic technology, Biomaterials 2011, 32, 7118-7126.

[Wet01] Wettig, S. D.; Verrall, R. E.; Thermodynamic Studies of Aqueous m-s-m Gemini Surfactant Systems, Journal of Colloid and Interface Science 2001, 235, $310-316$. 
[Whi20] Whitmore Smith, A.; John Tyndall (1820-1893), The Scientific Monthly 1920, 331-340.

[Whi01] Whitesides, G. M.; Stroock, A. D.; Flexible Methods for Microfluidics, Physics Today 2001, 42-47.

[Whi06] Whitesides, G. M.; The origins and the future of microfluidics, Nature 2006, $442,368-373$.

[Wie82] Wieczorek, P. P.; Ilavsky, M.; Kolarz, B. N.; Dusek, K.; Mechanical Behavior and Structure of Single Beads of Homogeneous and Macroporous StyreneDivinylbenzene Copolymers, Journal of Applied Polymer Science 1982, 27, 277-288.

[Wil75] Wiley, R. H.; Crosslinked Styrene/Divinylbenzene Network Systems, Pure and Applied Chemistry 1975, 43(1-2), 57-75.

[Wil88a] Williams, J. M.; Wrobleski, D. A.; Spatial Distribution of the Phases in Waterin-Oil Emulsions. Open and Closed Microcellular Foams from Cross-Linked Polystyrene, Langmuir 1988, 4, 656-662.

[Wil88b] Williams, J. M.; Toroidal Microstructures from Water-in-Oil Emulsions, Langmuir 1988, 4(1), 44-49.

[Wil89] Williams, J. M.; Wrobleski, D. A.; Microstructures and properties of some microcellular foams, Journal of Materials Science Letters 1989, 24, 4062-4067.

[Wil90a] Williams, J. M.; Gray, A. J.; Wilkerson, M. H.; Emulsion Stability and Rigid Foams from Styrene or Divinylbenzene Water-in-Oil Emulsions, Langmuir 1990, 6, 437-444.

[Wi190b] Williams, J. M., Bartos III, J. J.; Wilkerson, M. H.; Elastic modulus dependence on density for polymeric foams with systematic changing microstructures, Journal of Materials Science 1990, 25, 5134-5141.

[www1] https://goldbook.iupac.org/terms/view/M03667 (15.02.2021)

[www2] https://gestis.dguv.de/data?name=001180 (15.02.2021)

[www3] https://gestis.dguv.de/data?name=002310 (15.02.2021)

[www4] http://www.mathematische-basteleien.de/sechseck.htm (15.02.2021) 
[www5] https://www.wolframalpha.com/input/?i=rhombic + dodecahedron $\& x=0 \& y=0$ $(15.02 .2021)$

[Zha11] Zhao, C.-X.; Middelberg, A. P. J.; Two-phase microfluidic flows, Chemical Engineering Science 2011, 66, 1394-1411.

[Zha16] Zhang, J.; Yan, S.; Yuan, D.; Alici, G.; Nguyen, N.-T.; Warkian, M. E, Li, W.; Fundamentals and applications of inertial microfluidics: a review, Lab on a Chip 2016, 16, 10-34.

[Zhu16] Zhu, P.; Wang, L.; Passive and active droplet generation with microfluidics: a review, Lab on a Chip 2017, 17, 34-75.

[Zou90] Zou, D.; Derlich, V.; Gandhi, K.; Park, M.; Sun, L.; Kriz, D.; Lee, Y. D.; Kim, G.; Aklonis, J. J.; Salovey, R.; Model Filled Polymers. I. Synthesis of Crosslinked Monodisperse Polystyrene Beads, Journal of Polymer Chemistry: Part A: Polymer Chemistry 1990, 28, 1909-1921. 


\section{- 132 -}




\section{Declaration of Authorship}

I hereby certify that the dissertation entitled

Emulsion Templating: Unexpected Morphology of Monodisperse Macroporous

Polymers

is entirely my own work except where otherwise indicated. Passages and ideas from other sources have been clearly indicated.

Name: $\quad$ Lukas Koch

Signed:
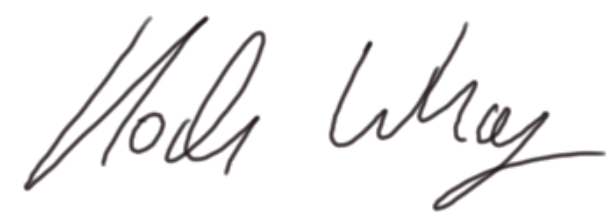

Date: $\quad 6^{\text {th }}$ of May 2021 


\section{$-134$}




\title{
Curriculum Vitae
}

\author{
Lukas Koch
}

born $28^{\text {th }}$ November 1993 in Bad Reichenhall, Germany

11/2017 - 03/2021 Institute of Physical Chemistry, University of Stuttgart

$\mathrm{PhD}$ Thesis (magna cum laude) „Emulsion Templating: Unexpected Morphology of Monodisperse Macroporous Polymers"

Two papers as leading author, two papers as co-author

Participation with presentation or poster at three national and two international conferences

Supervisor of three Bachelor theses and one lab internship, co-supervisor of one Master thesis

10/2015 - 09/2017 University of Regensburg

Master of Science $(1,2)$ in Complex Condensed Materials and Soft Matter

Master Thesis $(1,0)$ „Temperature-induced extraction and separation of lanthanides in pre-ouzo systems"

02/2017 - 07/2017 Institut de Chimie Séparative de Marcoule

Lab internship within the scope of the Master Thesis

10/2012 - 08/2015 University of Regensburg

Bachelor of Science $(1,9)$ in Chemistry

Bachelor Thesis $(1,0)$ „Extraktion von Rosmarinus officinialis L: Antioxidative Stärke von Extrakten“

09/2005 - 06/2012 Karlsgymnasium Bad Reichenhall

University Entrance Diploma $(1,2)$ in natural scientific and modern language branches 